\title{
The Isospin Dependence of Short Range Correlations through Inclusive Electron Scattering from ${ }^{40} \mathrm{Ca}$ and ${ }^{48} \mathrm{Ca}$
}

\author{
Dien Nguyen \\ Thanh Hoa, Vietnam
}

Bachelor in Physics, Hue University, 2010

\begin{abstract}
A Dissertation presented to the Graduate Faculty
of the University of Virginia in Candidacy for the Degree of

Doctor of Philosophy
\end{abstract}

Department of Physics

University of Virginia

December, 2018 


\section{Acknowledgments}

At the beginning of my thesis I want to express my appreciation to people who gave me so much support and help during my Ph.D. Without them, I would not be able to finish this thesis.

First of all, I would like to thank my thesis advisor, Prof. Donal Day, who couldn't be more supportive and encouraging. He was not only always there for me whenever I needed him, but he also believed in me and gave me enough freedom to be independent in my research. He also worked really hard on my bad writing to make this thesis understandable. I couldn't reach this point without his help and guidance. I deeply feel grateful to have him as my advisor.

I would like to thank Prof. P. Q. Hung, who is the father of the "Advanced Physics Program in Vietnam" which I attended as an undergraduate. That program really prepared me for the first steps of my Ph.D. study. Prof. Hung is the one who initiated my American dream and brought me to UVA. Without him, I couldn't be here to pursue my career.

I would like to thank my analysis supervisor, Dr. John Arrington. He is a true scientist who is extremely knowledgeable and patient. I learned a lot from him while working together. The discussions with him were always enjoyable and helped clear my confusions about the analysis. I also love the long emails from him which were very informative. Thank you so much for giving me your time and guidance even when you were super busy. I always feel lucky to be your student.

I would like to thank my JLab on-site supervisor, Dr. Douglas Higinbotham. He brought me to the lab and assigned me many useful tasks which really helped me be familiar with the experiment quickly. He also gave me a lot of guidance and valuable suggestions. He always believed in me and encouraged me to believe in myself. He cheered me up whenever I felt down or doubted myself. Thank you so much for taking care of me during this long path. 
I would like to thank Prof. Nilanga Liyanage, Prof. Blaine Norum and Prof. Trinh Thuan for their time and agreement to be my thesis committee members.

I spent several years of my Ph.D. study at JLab, during which I was so lucky to have the opportunity to work with scientists at the lab or from other universities. I would like to thank Dr. Christian Weiss, Prof. Eric Christy, and Dr. Alexandre Camsonne. I learned a great deal from each of them. They gave me an open view of research areas outside of my Ph.D. study. I also want to thank Dr. David Mack. He is such a nice person who always cared about me and spent a significant amount of time guiding me through my thesis writing. Without his help, I don't think I could finish my thesis on time.

I am so happy and grateful to have the opportunity to work with the amazing tritium group, Argon group, Gmp group and many postdocs at the lab. They have been great coworkers, giving me so much support and help. I would like to thank Dustin Keller, Luke Myers, Jixie Zhang, Florian Hauenstein, R.Evan McClellan, Marco Carmignotto and Zhihong Ye for their help and advice. In particular, I want to say thank you very much to Florian, who spent a lot of time on my thesis even when he was busy with the run coordinator duty. I want to say thank you very much to every group member, including Sheren Alsalmi, Tong Su, Shujie Li, Hanjie Liu, Tyler Hague, Mike Nycz, Jason Bane, Rey Torres, Yang Wang, Hongxia Dai, Mikhail Yurov, Daniel Abrams, Nathaly Santiesteban and Jessica Campbell. You are not only great coworkers but also good friends and my family here. Thank you so much for walking with me along this path. I wish you guys all the best. I want to say thank you so much to Barak Schmookler. He has been so patient with any questions I had for him since I moved to JLab and always tried to help me solve my problems. Our friendship means a lot to me. I wish you all the best in your next steps.

I would like to thank Vinh Hoang, Long Trinh, Hanh Le, Nguyen Ton, Toan Bui, Dung Ho, Kha Tran, Trinh Le, Dat Duong, Truc Le for all the good time we 
had together back in school. I will never forget those days. I always remember and appreciate the support from my friends at UVA, Moran Chen, Luna Yang, Keija Li, and those friends at JLab, Carlos Gayoso, Zongwen Yang, Longwen Ou, Lei Xing, Chao Gu, Chao Peng, Ying Jin, Li Ye and Qian Zhang. Thank you so much for taking care of me. Still there are a lot of people who I want to say thank you to but I could not explicitly mention here.

Finally, I want to say thank you so much to my parents, my brothers and sisters. I am the youngest kid in my family and they really did not want me to go so far away from the hometown. However, they always support me to do what I want. I am so lucky to be your daughter, and little sister. I love you very much. I also want to say thank you very much to my American grandparents, Lon Holmberg and Sandy Stillwell, who have made me feel loved and warm since I moved to US. Special thanks go to my boyfriend, Longwu Ou, for his love, understanding and support. I am lucky to have you. 


\begin{abstract}
Short Range Correlations (SRCs) have been recognized as responsible for the high momentum tail of the nucleon momentum distribution in nuclei and are necessary to explain the missing nuclear strength in the mean field theory. One of the important questions about SRCs is their isospin dependence, yet unseen in inclusive scattering. An experiment (E08014) took place in Hall A at Jefferson Lab in Spring 2011 aimed to study SRCs. The inclusive electron scattering cross-sections from different nuclei including ${ }^{2} \mathrm{D},{ }^{3} \mathrm{He},{ }^{4} \mathrm{He},{ }^{12} \mathrm{C},{ }^{40} \mathrm{Ca}$ and ${ }^{48} \mathrm{Ca}$ were measured in kinematic region of $\left(0.8<Q^{2}<2.8 \mathrm{GeV}^{2}\right)$ and $\left(1<x_{b j}<2\right)$ where SRCs are expected to be dominant $\left(x_{b j}=Q^{2} / 2 m_{p} \nu, Q^{2}\right.$ is the 4-momentum transfer square, and $\nu$ is the transfer energy). The cross section ratios of ${ }^{4} \mathrm{He}$ nuclei to ${ }^{3} \mathrm{He}$ for $x_{b j}>2$ were used to search for the presence of 3N SRCs. The analysis herein focuses on the isospin dependence of SRCs using the cross section ratio per nucleon of ${ }^{48} \mathrm{Ca}$ to ${ }^{40} \mathrm{Ca}$.
\end{abstract}




\section{Contents}

$\begin{array}{lll}1 & \text { Introduction } & 11\end{array}$

1.1 Overview . . . . . . . . . . . . . . . . . . . 11

1.2 Independent Particle Shell Model . . . . . . . . . . . . . . . . . . . . 14

1.3 Indirect evidence of SRCs $\ldots \ldots \ldots \ldots$

1.4 The Origin and Features of SRCs . . . . . . . . . . . . . . . . 22

1.5 Inclusive Electron Scattering . . . . . . . . . . . . . . . . . . 25

1.5.1 Quasi-elastic scattering . . . . . . . . . . . . . 28

1.5 .2 Where to find SRCs in Inclusive Scattering . . . . . . . . . . . 31

\begin{tabular}{lll}
\hline 2 & SRCs measurements & 36
\end{tabular}

2.1 Results of Inclusive experiments . . . . . . . . . . . . . . . . 36

2.2 Results of Coincident Experiments . . . . . . . . . . . . . . . . . 42

2.3 SRCs and The EMC effect . . . . . . . . . . . . . . . . . . . . . . . 48

2.4 Motivations of Experiment E08-014 . . . . . . . . . . . . . . . . 51

\begin{tabular}{|lll}
\hline 3 & The Experiment Setup & 56
\end{tabular}

3.1 Overview. . . . . . . . . . . . . . . . . . . 56

$3.2 \quad \mathrm{E} 08014 \ldots \ldots \ldots \ldots \ldots \ldots$

3.3 CEBAF $\ldots \ldots \ldots \ldots \ldots \ldots$

3.4 Beam Line . . . . . . . . . . . . . . . . . . . . . . . . . . . . . . . . 59

3.4 .1 Beam Position Monitors . . . . . . . . . . . . . . . . . . . . . 59 
3.4 .2 Raster . . . . . . . . . . . . . . . . . . 60

3.4 .3 Beam Current Monitor (BCMs) . . . . . . . . . . . . . . 61

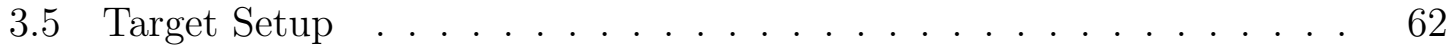

3.6 Spectrometers $\ldots \ldots \ldots \ldots$

3.6 .1 Vertical Drift Chambers . . . . . . . . . . . . . 66

3.6 .2 Scintillators . . . . . . . . . . . . . . . 68

3.6 .3 Gas Cherenkov . . . . . . . . . . . . . . . . . . . . 69

3.6 .4 Calorimeters . . . . . . . . . . . . . . . . . 71

$\begin{array}{lllll}3.6 .5 & \text { Trigger Design } & \ldots & \ldots & \ldots\end{array} \ldots \ldots \ldots$

\begin{tabular}{|lll}
4 & Data Analysis & 74
\end{tabular}

4.1 Overview. . . . . . . . . . . . . . . . . . . . . . . . . . . . . . . . . 74

4.2 Beamline Calibration . . . . . . . . . . . . . . . . . . . . . 74

4.2 .1 BPM calibration . . . . . . . . . . . . . . 74

4.2 .2 Raster Calibration . . . . . . . . . . . . . . . . . . 79

4.3 Detector calibration . . . . . . . . . . . . . . . . . . . . . 84

4.3 .1 VDC Calibration . . . . . . . . . . . . . . . . 84

4.3 .2 Cerenkov's ADC calibration . . . . . . . . . . . . . 86

4.3 .3 Calorimeter Calibration $\ldots \ldots \ldots \ldots$

4.4 Optics Calibration $\ldots \ldots \ldots \ldots$

$4.4 .1 \quad$ HRS coordinate systems $\ldots \ldots \ldots \ldots$

4.4 .2 Optimization procedure $\ldots \ldots \ldots \ldots \ldots$

$4.4 .3 \quad$ Reconstruction after optimization $\ldots \ldots \ldots \ldots$

4.4 .4 Performance of $\mathrm{G}_{M}^{p}$ optics on E08014 data . . . . . . . . 99

$4.5 \quad$ Efficiency Studies $\ldots \ldots \ldots \ldots \ldots$

4.5 .1 Beam Charge Calculation . . . . . . . . . . . . . 103

4.5 .2 Livetime . . . . . . . . . . . . . . . . . . . . . 105

$4.5 .3 \quad$ Particle Identification Efficiency $\ldots \ldots \ldots \ldots \ldots$ 
4.5 .4 Tracking Efficiency $\ldots \ldots \ldots \ldots$

4.6 Acceptance Study . . . . . . . . . . . . . . . . . . . . . . . . . . . . 122

4.6 .1 Simulation (SAMC) . . . . . . . . . . . . . . . . . . 122

4.6 .2 Pointing Study . . . . . . . . . . . . . . . . . . . . . . 124

4.6 .3 Cross section Model (XEMC) $\ldots \ldots \ldots$

4.6 .4 Data and simulation comparison. . . . . . . . . . . 130

$\begin{array}{lll}5 & \text { Cross Section Analysis } & 133\end{array}$

$5.1 \quad$ Overview . . . . . . . . . . . . . . . . . . . . . . 133

5.2 Two Methods to extract the cross section . . . . . . . . . . . . . . 134

5.3 Yield ratio for overlap kinematics $\ldots \ldots \ldots \ldots \ldots$

5.4 Local efficiency issue . . . . . . . . . . . . . . . . . . . . . 139

$5.5 \quad$ Acceptance dependence $\ldots \ldots \ldots \ldots \ldots \ldots$

$5.6 \quad$ Yield ratio for ${ }^{40} \mathrm{Ca}$ and ${ }^{48} \mathrm{Ca} \ldots \ldots \ldots \ldots \ldots$

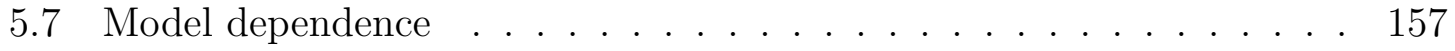

5.8 Systematic uncertainty estimation $\ldots \ldots \ldots \ldots \ldots \ldots$

6 Results and discussion 162

6.1 Absolute Cross section . . . . . . . . . . . . . . . . . . . . . 162

6.2 Cross section ratio ${ }^{48} \mathrm{Ca} /{ }^{40} \mathrm{Ca} \ldots \ldots \ldots \ldots \ldots \ldots$

6.3 Discussion $\ldots \ldots \ldots \ldots \ldots$

6.4 Outlook $\ldots \ldots \ldots \ldots \ldots \ldots \ldots$ 


\section{List of Figures}

1.1 The proton and neutron potential well . . . . . . . . . . . . . 15

1.2 The effective potential of IPSM $\ldots \ldots \ldots$

1.3 Magic numbers in nuclei . . . . . . . . . . . . . . . . . . . . . . . 17

1.4 The Be spectral function . . . . . . . . . . . . . . . . . . . 18

1.5 The electron scattering $A\left(e, e^{\prime} p\right)$ diagram . . . . . . . . . . . . . 19

1.6 The momentum distribution results from the $A\left(e, e^{\prime} p\right), \mathrm{NIKHEF}$. . . 20

1.7 The spectroscopic factor for valance shell from $A\left(e, e^{\prime} p\right)$, NIKHEF-K. 21

1.8 Caption . . . . . . . . . . . . . . . . . . . . . . 23

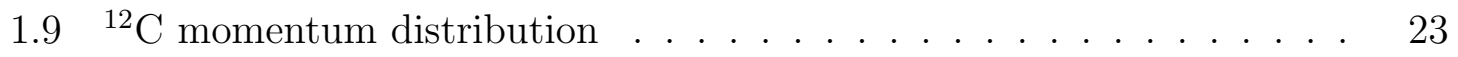

1.10 Momentum distribution comparison . . . . . . . . . . . . . . . . . . 24

1.11 The momentum distribution for different nuclei . . . . . . . . . . . . 25

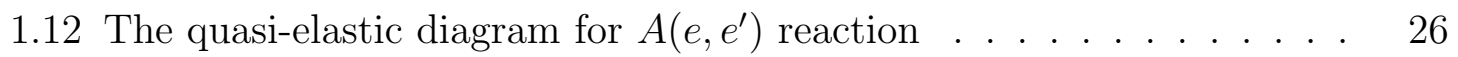

1.13 The inclusive cross section for electron scattering off a nucleus . . . . 27

1.14 The ${ }^{12} \mathrm{C}$ extracted $F(y)$ from E89-008 . . . . . . . . . . . . . . . . . . 31

1.15 The minimum momentum of struck nucleon . . . . . . . . . . . . . 33

1.16 The inclusive cross section model . . . . . . . . . . . . . . . . . . . . 34

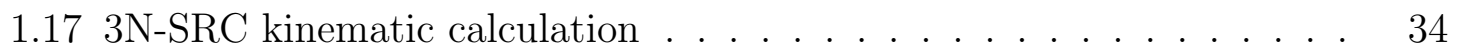

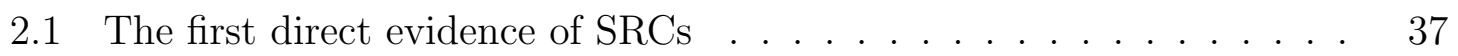

2.2 The Hall B JLab, 2N SRCs CLAS data . . . . . . . . . . . . . . . . . 39 
2.3 The Hall B JLab, CLAS data $\ldots \ldots \ldots \ldots \ldots$

2.4 The Hall C JLab, E02-019, 2N SRCs data . . . . . . . . . . . . 41

2.5 The Hall C JLab, E02-019, 3N SRCs data . . . . . . . . . . . . 41

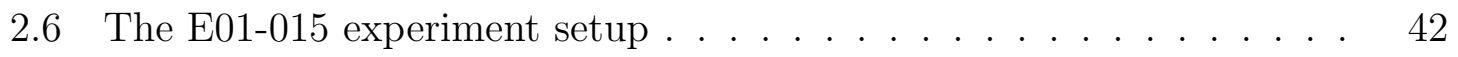

2.7 The $\cos \left(\mathbf{p}_{\text {miss }}, \mathbf{p}_{\text {rec }}\right)$ distribution $\ldots \ldots \ldots \ldots \ldots \ldots$

2.8 The isospin dependence evidence results from coincident experiment . 44

2.9 Tensor dominance in the nucleon momentum distribution . . . . . . . 45

2.10 The isospin-dependence in different nuclei $\ldots \ldots \ldots$

2.11 The momentum distribution assumption in contact formalism . . . . 47

2.12 The average kinetic energy for different nuclei $\ldots \ldots \ldots \ldots$

2.13 The $F_{2}^{F e} / F_{2}^{D}$ from data at Cern $\ldots \ldots \ldots \ldots \ldots \ldots$

2.14 The EMC effect study at SLAC and JLab . . . . . . . . . 50

2.15 The connection between EMC effect and SRCs . . . . . . . . . . 51

2.16 The 3 N SRCs studies $\ldots \ldots \ldots \ldots \ldots$

2.17 Theoretical prediction if the isospin dependence $\ldots \ldots \ldots \ldots$

3.1 The kinematic coverange $\ldots \ldots \ldots \ldots \ldots \ldots$

3.2 Hall A CEBAF $\ldots \ldots \ldots \ldots \ldots \ldots \ldots$

3.3 Hall A Beam line . . . . . . . . . . . . . . . . . . . . . . . . . . 59

3.4 Hall A BPMs $\ldots \ldots \ldots \ldots \ldots \ldots \ldots$

3.5 Hall A BCMs layout $\ldots \ldots \ldots \ldots \ldots$

$3.6 \quad$ The target configuration for 2 periods $\ldots \ldots \ldots \ldots \ldots$

$3.7 \quad$ Hall A magnet system for HRS $\ldots \ldots \ldots \ldots \ldots$

3.8 Hall A HRS detector package $\ldots \ldots \ldots \ldots \ldots \ldots$

3.9 The lay-out of Vertical Drift Chambers for one HRS . . . . . . . . . 69

3.10 Vertical Drift Chambers for one HRS . . . . . . . . . . . . . 70

3.11 Hall A Scintillator Diagram $\ldots \ldots \ldots$ 
3.12 A diagram of the Gas Cherenkov showing the spherical mirrors focusing the cherenkov light to $10 \mathrm{PMTs}$. . . . . . . . . . . . . . . . . . . 71

3.13 The Calorimeter lay-out . . . . . . . . . . . . . . . . . . 72

3.14 Trigger design . . . . . . . . . . . . . . . . . . . . . . . 73

4.1 BPMs convention . . . . . . . . . . . . . . . . . . . . 76

4.2 Beam X convention in Hall A coordinate system . . . . . . . . . . . . 76

$4.3 \mathrm{Y}_{t g}$ distribution of the Bulls-eyes scan . . . . . . . . . . . . . 77

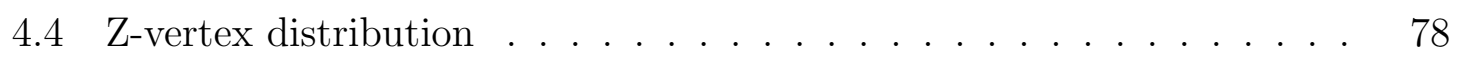

4.5 Phase lag in the beam position . . . . . . . . . . . . . 80

4.6 Raster Calibration at Target location . . . . . . . . . . . . . . . 80

4.7 Determining the sign of $K_{x}$ for raster calibration . . . . . . . . . . . 81

4.8 Select the events from central foils . . . . . . . . . . . . . . . . . . . . 82

4.9 Select the events from the central hole of the sieve . . . . . . . . . . . 83

4.10 Determining the sign of $K_{y}$ for the raster calibration . . . . . . . . . 83

4.11 The VDC rawtime distribution . . . . . . . . . . . . . . . . . . . 84

4.12 The VDC corrected time . . . . . . . . . . . . . . . . 85

4.13 The SPE location before and after calibration for PMT1. . . . . . . . 87

4.14 The PMT3 spectrum . . . . . . . . . . . . . . . . . . 87

4.15 The SPE alignment using initial Cherenkov calibration . . . . . . . . 88

4.16 The SPE alignment with new calibration. . . . . . . . . . . . . . . . . 89

4.17 The $\mathrm{E} / \mathrm{p}$ for different kinematics . . . . . . . . . . . . . . . . . 90

4.18 Hall coordinate system (HCS) (top view). . . . . . . . . . . . . . 92

4.19 Target coordinate system for top view and side view. . . . . . . . . . 93

4.20 Detector coordinate system, top and side view. . . . . . . . . . . . 93

4.21 Transport coordinate system (side view). . . . . . . . . . . . . . 94

4.22 Focal plane coordinate system (side view). . . . . . . . . . . . . . 95 
$4.23 \mathrm{Z}_{\text {vertex }}$ after the optimization in $\mathrm{G}_{M}^{p}$ optics using Spring 2016 optics data. The blue lines are the expected values. The black curves are the event distribution for different target foils. . . . . . . . . . . . . . . . 99

4.24 Reconstructed angle after the optimization in $G_{m}^{P}$ optics using Spring

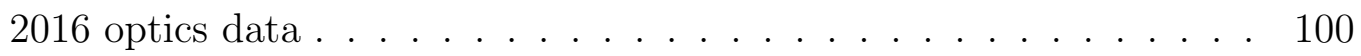

4.25 Relative momentum reconstruction after the optimization in $G_{m}^{P}$ optics

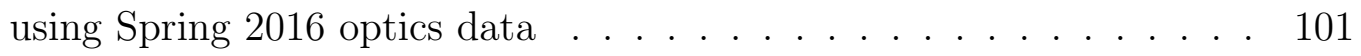

4.26 Multiple foils target, Y reconstruction . . . . . . . . . . . . . . . . . . 101

4.27 Multiple foils target, in plane angle vs Ytg reconstruction. The foils are well separated and the reconstructed positions agree well with the expected position of each foil. . . . . . . . . . . . . . . . . . . . . . . 102

4.28 Sieves run with multiple foils target. The pattern of the sieve for the central foil. . . . . . . . . . . . . . . . . . . . 102

4.29 The number of BCM counts from bcm-u1 vs number of count from the clock. When beam trips, the bcm count doesn't change. . . . . . . . . 103

4.30 Cherenkov and $\mathrm{E} / \mathrm{p}$ distribution . . . . . . . . . . . . . . 107

4.31 Cherenkov and $\mathrm{E} / \mathrm{p}$ distribution in $2 \mathrm{D}$. . . . . . . . . . . . . . 108

4.32 Select event for PID study . . . . . . . . . . . . . . . . . . . . . . 109

4.33 Select event using Calorimeter . . . . . . . . . . . . . . . . . 110

4.34 Electron and pion samples using the calorimeter information in 2D . 110

4.35 Electron and pion samples using the calorimeter information in 1D . 111

4.36 Cherenkov sum cut efficiency . . . . . . . . . . . . . . . . . . . . . . . 112

4.37 Electron sample on the Cherenkov sum . . . . . . . . . . . . . . . . 113

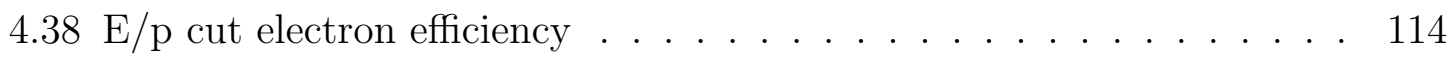

4.39 Wire efficiency . . . . . . . . . . . . . . . . . . . . . 116

4.40 Cluster in a wire plane . . . . . . . . . . . . . . . . . . . . . . 117

4.41 The global track using two cross-over points . . . . . . . . . . . . . . 117 
4.42 Number of cluster for Zero track and Multiple track . . . . . . . . . . 118

4.43 The track number distribution in the selected sample . . . . . . . . . 119

4.44 SAMC aperture check . . . . . . . . . . . . . . . . . . . . . . . 123

4.45 SAMC package structure . . . . . . . . . . . . . . . . . 124

4.46 The convention for mis-pointing study in the Lab and the target coor-

dinator system. . . . . . . . . . . . . . . . . . 125

4.47 The contribution of the beam $\mathrm{X}$ to the $\mathrm{Y}_{t g} \ldots \ldots$. . . . . . . . 127

4.48 The contribution of the target offset to the $\mathrm{Y}_{t g} \ldots \ldots \ldots$. . . . . . 128

4.49 XEMC package structure . . . . . . . . . . . . . . . . . . . . 130

4.50 Data, simulation comparison using initial optics . . . . . . . . . . . . 131

4.51 Data, simulation comparison using GMP optics . . . . . . . . . . . . 132

4.52 Data, simulation comparison after included every correction . . . . . 132

5.1 Yield ratio for overlap kinematics as function of $\mathrm{E}_{p}$. . . . . . . . . . 138

5.2 Yield ratio for overlap kinematics as function of $\mathrm{x}_{b j}$. . . . . . . . . . 138

5.3 2D Yield ratio as function of in plane angle $\phi_{t g}(\mathrm{rad})$ vs out plane angle $\theta_{t g}(\mathrm{rad})$ shows a uniform distribution except few points at the edge where acceptance was not modeled well. . . . . . . . . . . . . . 140

5.4 The 2D Yield ratio as function of in plane angle $\phi_{t g}(\mathrm{rad})$ vs relative momentum dp shows a specific region where the yield ratio drops. This occurs at $\mathrm{dp} \sim 0.03$ and $\phi_{t g}>-0.005$. . . . . . . . . . . . . . . . 141

5.5 The Trigger 3 event distribution on the Cherenkov phase space . . . . 141

5.6 The trigger 7 events distribution on the Cherenkov phase space . . . 142

5.7 The location of each of the 10 PMTs in the Cherenkov phase space • 142

5.8 The local efficiency as function of cherenkov position . . . . . . . . . 143

5.9 The local efficiency as function of $\mathrm{dp}$ or $\mathrm{x}_{b j} \ldots \ldots \ldots$. . . . . . . . . 144

5.10 The local efficiency as function of dp for all kinematic settings . . . . 145

5.11 The Yield ratio for overlap region as function of $E^{\prime}$ after correction . 146 
5.12 The Yield ratio for overlap region as function of $\mathrm{x}_{b j}$ after correction . 146

5.13 Comparison between two ways of the local efficiency correction . . . . 147

5.14 The acceptance dependence as a function of $\mathrm{E}^{\prime}$ for $\theta_{0}=21^{\circ}$ and $\theta_{0}=$ $23^{\circ}$ settings $\ldots \ldots \ldots \ldots \ldots \ldots \ldots \ldots$

5.15 The acceptance dependence as a function of $\mathrm{E}^{\prime}$ for $\theta_{0}=25^{\circ}$ settings . 149

5.16 The acceptance dependence as a function of $x_{b j}$ for $\theta_{0}=21^{\circ}$ and $\theta_{0}=$

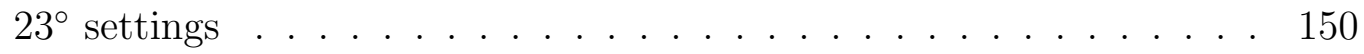

5.17 The acceptance dependence as a function of $\mathrm{x}_{b j}$ for $\theta_{0}=25^{\circ}$ settings . 150

5.18 The initial ${ }^{40} \mathrm{Ca}$ and ${ }^{48} \mathrm{Ca}$ yield ratio as a function of $\mathrm{E}^{\prime}$. . . . . . . 152

5.19 The initial ${ }^{40} \mathrm{Ca},{ }^{48} \mathrm{Ca}$ Yield ratio as a function of $\mathrm{x}_{b j} \ldots \ldots \ldots$

5.20 The initial extracted ${ }^{40} \mathrm{Ca}$ cross section . . . . . . . . . . . . . 153

5.21 The initial extracted ${ }^{48} \mathrm{Ca}$ cross section . . . . . . . . . . . . . . 153

5.22 New $\mathrm{F}(\mathrm{y})$ fit on the initial ${ }^{40} \mathrm{Ca}$ cross section . . . . . . . . . . 154

5.23 New $\mathrm{F}(\mathrm{y})$ fit on the initial ${ }^{48} \mathrm{Ca}$ cross section . . . . . . . . . . 155

5.24 The initial ${ }^{40} \mathrm{Ca}$ and ${ }^{48} \mathrm{Ca}$ yield ratio as a function of $\mathrm{E}^{\prime} \ldots \ldots \ldots$

5.25 The initial ${ }^{40} \mathrm{Ca},{ }^{48} \mathrm{Ca}$ Yield ratio as a function of $\mathrm{x}_{b j} \ldots \ldots \ldots$

\begin{tabular}{|lll}
5.26 The initial and the first iterated ${ }^{40} \mathrm{Ca}$ cross section model comparison & 157
\end{tabular}

5.27 The initial and the first iterated ${ }^{48} \mathrm{Ca}$ cross section model comparison 158

5.28 The ${ }^{40} \mathrm{Ca}$ and ${ }^{48} \mathrm{Ca}$ model dependence effects as a function of $\mathrm{E}^{\prime}$. . . 159

5.29 The ${ }^{40} \mathrm{Ca}$ and ${ }^{48} \mathrm{Ca}$ model dependence effects as a function of $x_{b j} \ldots 160$

5.30 The $d p: \phi_{t g}$ phase space for each $\mathrm{x}_{b j}$ bin $\ldots \ldots \ldots \ldots \ldots$

$6.1{ }^{12} \mathrm{C}$ absolute cross-section $\ldots \ldots \ldots \ldots \ldots$

$6.2{ }^{40} \mathrm{Ca}$ absolute cross-section . . . . . . . . . . . . . . . . . . 163

$6.3{ }^{48} \mathrm{Ca}$ absolute cross-section . . . . . . . . . . . . . . . . . . . . 164

6.4 The cross-section ratio ${ }^{48} \mathrm{Ca} /{ }^{40} \mathrm{Ca}$ per nucleon for $\theta_{0}=21^{\circ} \ldots \ldots$

6.5 The cross-section ratio ${ }^{48} \mathrm{Ca} /{ }^{40} \mathrm{Ca}$ per nucleon for $\theta_{0}=23^{\circ} \ldots \ldots 6$

6.6 The cross-section ratio ${ }^{48} \mathrm{Ca} /{ }^{40} \mathrm{Ca}$ per nucleon for $\theta_{0}=25^{\circ} \ldots . . .66$ 


\section{List of Tables}

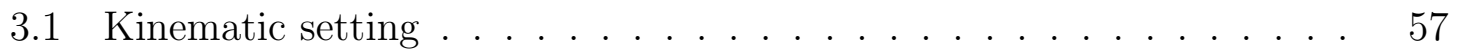

3.2 The Cryogenic target configuration for two run periods $\ldots \ldots$. . . . 62

3.3 The operating information for Cryogenic Targets, Period 1 . . . . . 63

3.4 The operating information for Cryogenic Targets, Period 2 . . . . . 64

3.5 The encoder position of each target on the Target ladder . . . . . . . 64

3.6 The window thickness information for Cell targets $\ldots \ldots \ldots$. . . . 64

3.7 Solid Targets information $\ldots \ldots \ldots \ldots \ldots \ldots$

3.8 The characteristics of Hall A where the resolution values listed are FWHM. See Ref. [59]] . . . . . . . . . . . . . . . . . . 68

4.1 Bulls-eye Scan Data . . . . . . . . . . . . . . . . . . . . . 75

4.2 BPMs coefficients from an early Calibration for E08-014, Ref. [54] . . 77

$4.3 \quad$ Particle Identification efficiency summary . . . . . . . . . . . . . . . 114

4.4 Tracking efficiency for zero, single, multiple track for each kinematic . 121

$4.5 \quad$ SAMC apertures check list . . . . . . . . . . . . . . . . . . 123

$4.6 \quad$ Survey results for Spectrometer mis-pointing [88] $\ldots \ldots \ldots \ldots \ldots$

4.7 Summary of Pointing study through every run in XGT2 data. . . . . 129

5.1 The sets of acceptance cuts on the target reconstructed variables . . . 148

5.2 Systematic Uncertainty summary $\ldots \ldots \ldots \ldots \ldots$

$6.1 \quad$ Theoretical $a_{2}(A)$ calculation for ${ }^{40} \mathrm{Ca}$ and ${ }^{48} \mathrm{Ca} \ldots \ldots \ldots$ 


\section{Chapter 1}

\section{Introduction}

\subsection{Overview}

In 1897, J. J. Thomson discovered the electron through the magnetic deflection of cathode rays; the result indicated the atom has an internal structure [1]. A decade after that, in 1910, the Ernest Rutherford's team discovered the nucleus by performing an experiment, passing alpha particles through a thin gold foil and observed that a few particles were scattered through large angles, even completely backward in some cases [2, 3]. This remarkable result indicated the atom has a very small and dense nucleus containing most of its mass. In 1932 the neutron was discovered by James Chadwick [4]. These breakthrough discoveries mark the start of nuclear physics.

Since nuclear physics was established, the main research goal is a complete description of nuclear structure which requires the understanding of the interaction between nucleons (protons or neutrons) and how these interactions determine nuclear properties. In a simple picture, the existence of stable nuclei is evidence that the nucleon-nucleon $(\mathrm{N}-\mathrm{N})$ interaction must be attractive, and much stronger then the Coulomb forces, to keep the protons bound. However, it cannot be attractive over all distances because the short distance $\mathrm{N}-\mathrm{N}$ interaction must be repulsive to keep the 
nucleus from collapsing [5].

The complete description of the nuclear structure can be obtained by solving a many-body problem. This is very challenging due to the complexities of the nuclear system. The complexity level increases with $A$. The solution of this problem is only available for the few-body systems, and up to $A=12\left({ }^{12} \mathrm{C}\right)[6]$. For heavy nuclei, beyond ${ }^{12} \mathrm{C}$, this problem was and still is nearly impossible. Many effective models have been developed in an attempt to describe nuclear structure and they all have to apply different approximations in order to simplify the many-body problem. The most successful model for nuclear structure is the independent particle shell model (IPSM) invented by Goeppert-Mayer and Jensen in 1949. The IPSM can predict surprisingly well the properties of nuclei such as their angular momentum, parity, magic number, spin, etc. In the early 1980's, A(e, e'p) experiments at NIKHEF-K revealed a significant discrepancy between IPSM model predictions for the strength of valence-shell protons when compared to data (30\% - 40\%) [7]. More details are discussed in the next sections.

One of the possible way to remedy and understand this discrepancy is the existence of short range correlations (SRCs) which are not included in the IPSM. SRCs refers to two or more nucleons coming together at a short distances where their wave functions overlap. The SRCs are assumed to be responsible for the high momentum tail of the nucleon momentum distribution. Finding the fraction of nucleon in SRCs, as compared to the fraction of nucleon in mean field gives a better understanding of short-distance ground state nuclear structure. An area of great interest is the isospin dependence of SRCs. That is, are the two nucleon SRCs pairs more or less likely to be iso-singlet ( $\mathrm{np}, \mathrm{T}=0$ ), iso-triplet ( $\mathrm{pp}, \mathrm{nn}, \mathrm{np}, \mathrm{T}=1$ ) pairs? The analysis presented in this thesis may provide a partial answer. The Calcium isotopes ${ }^{40} \mathrm{Ca}$ and ${ }^{48} \mathrm{Ca}$ provide an ideal test of isospin dependence; while they have equal number of protons, ${ }^{48} \mathrm{Ca}$ has 8 more neutrons. 
The electron is an ideal probe for studying nuclear structure. It is a structureless particle which interacts with the nucleus via the well-understood electromagnetic interaction. The relative weakness of the electromagnetic interaction not only enables one to model the process by the exchange of a single virtual photon, but it is also allows the electron to probes the entire nucleus. The resolution of electron probe is defined by the wavelength of the virtual photon; smaller wavelength (larger momentum transfer) corresponds to finer resolution. The singular disadvantage of electron scattering is its small cross section which requires a high intensity electron beam and thick targets. In addition, high momentum transfer $(q)$ is required to provide the resolution neccesary to study nuclear structure at short distances. These requirements were a challenge for SRCs studies with accelerators available in the 1980's.

Fortunately, the development in accelerator technologies provides much higher beam energy and luminosity which, together with much better theoretical understanding of nuclear structure, allowed a new generation of SRCs measurements. In the last 30 years, many electron scattering experiments focused on SRCs have been completed and many important results have been achieved. A big transition has been made and SRCs physics has evolved from searching for their existence to mapping their strength in nuclei, to studying their isospin dependence, exploring the possible connections between SRCs and the EMC effect, and more. A summary of the main results of previous SRCs measurements along with the motivation of this thesis experiment is presented in Chapter 2. Experiment E08014's setup and a summary of kinematic settings are described in Chapter 3. The analysis details are discussed in Chapter 4. The procedure of extracting cross section is given in Chapter 5. The results and discussion can be found in Chapter 6 . 


\subsection{Independent Particle Shell Model}

As mentioned above, the complete description of nuclei can be obtained by solving a many-body problem [5, 8, 9]. The Hamiltonian is defined as:

$$
H=T+\sum_{i<j}^{A} V_{2-b o d y}(i, j)+\sum_{i<j<k}^{A} V_{3-b o d y}(i, j, k)+\ldots
$$

where $T$ is the kinetic energy, $V_{2-b o d y}$ and $V_{3-b o d y}$ are the two and three-nucleon potentials, respectively. The conditions $(i<j)$ and $(i<j<k)$ avoid double counting of the interaction energy. The main assumption made in the shell model is that the nucleons move independently of each other in well-defined quantum orbits in the average potential (mean field $V_{M}$ ) created by the surrounding nucleons and that other interactions are neglected. This approximation significantly reduces the complexity of the many-body problem to that of a single particle in a potential well. This can be expressed as:

$$
H=\underbrace{\left[T+V_{M}\right]}_{\text {IPSM }}+\underbrace{\left[V_{2-\text { body }}+V_{3-\text { body }}+\ldots-V_{M}\right]}_{\text {neglected in IPSM }} .
$$

The nucleus is a dense system so it is reasonable to expect that there are substantial interactions between the nucleons inside the nucleus. This raises a question of why the IPSM works so well. The explanation relies heavily on the Pauli principle: the scattering of nucleons to occupied states is forbidden which suppresses the interaction between nucleons. For a given ground state nucleus, nucleons occupy independent shells and fill up distinct energy levels. The highest energy level is called the Fermi energy corresponding to the highest momentum, the Fermi momentum $k_{F}$. In other words, no nucleon above the Fermi energy and momentum are allowed in the IPSM. The IPSM also assumed that the protons and neutrons have independent shell states. The potential experienced by the proton needs to include the repulsive Coulomb 
potential [8]. A sketch of neutron and proton potentials well can be seen in Figure 1.1. The effective potential used in the IPSM is the Woods-Saxon potential with spin-orbit term (see Refs. [5,8]) which can be expressed as:

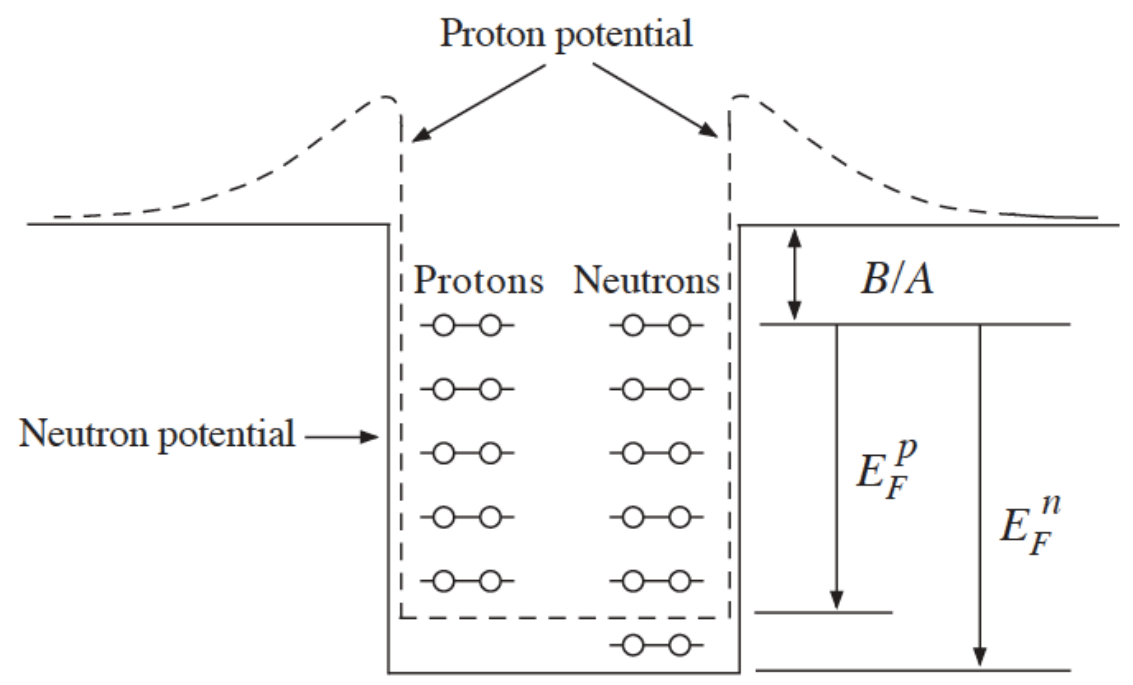

Figure 1.1: The protons and neutrons have independent potentials. The proton potential is the sum of the neutron potential and Coulomb potential. The difference from the top of the potential well and the highest energy level is the average binding energy per nucleon [5].

$$
V_{M}=\underbrace{\left[\frac{-V_{0}}{1+\exp [(r-R) / a]}\right]}_{\text {Woods-Saxon potential }}+\underbrace{\left[V_{l s}(r) \mathbf{L} \cdot \mathbf{S}\right]}_{\text {Spin-orbit interaction term }},
$$

where $V_{0}, a$ and $R$ are constants; $V_{0} \sim 50 \mathrm{MeV}, a \sim 0.5 \mathrm{fm}, R=1.25 A^{1 / 3} \mathrm{fm} 8$. The $\mathbf{L}$ and $\mathbf{S}$ are orbital and spin angular momentum operators, respectively. The shape of the IPSM potential and the spin-orbit effects can be seen in Figure 1.2. The spin-orbit interaction has no effect when $l=0$, is more attractive interaction when $l$ and $s$ are parallel and less attractive when $l$ and $s$ are anti-parallel [8, 10, 11. This spin-orbit term is the main key to be able to understand the shell structure of the IPSM. Without the spin-orbit term, the energy states do not depend on the total angular momentum, defined as $\mathbf{J}=\mathbf{L}+\mathbf{S}$. For a given energy level the number of 
states a nucleons can occupy is $2(2 l+1)$ where $l$ is the angular momentum quantum number which only can describe the first 3 magic numbers. Including the spin-orbit term, the energy levels are further split. The number of nucleons which can occupy a given energy level is $(2 j+1)$ where $j$ is the total angular momentum quantum number. This then reproduces all magic numbers (see Figure 1.3). A more detailed discussion can be found in Ref. [5]. In order to understand IPSM predictions, the spectral function which describes the energy and momentum distributions and the occupancy of a given shell are discussed next.

(a) The Woods-saxon potential

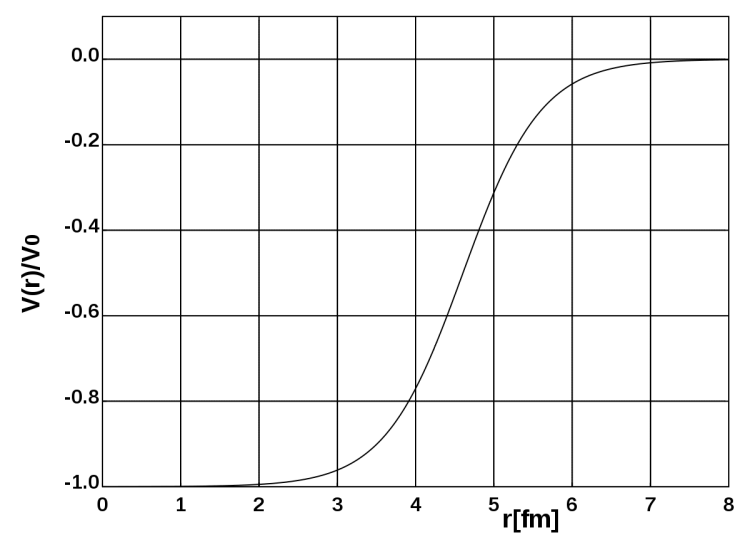

(b) The spin-orbit effect

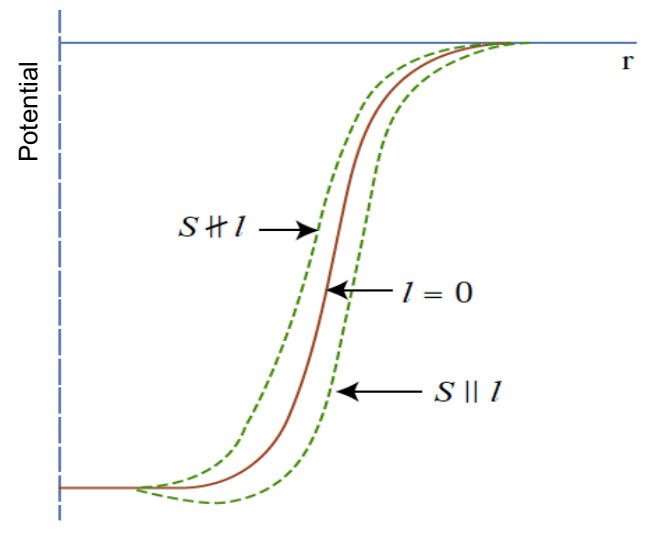

Figure 1.2: The effective potential in the independent particle shell model. Figure (a) [11] is the shape of the Woods-Saxon potential for $A=50$ as a function of distance. At a distance below $2 \mathrm{fm}$, the potential is flat which indicates no interaction. Figure (b) 10 shows the spin-orbit effects on the potential. There is no effect when $l=0$, more attractive when $s$ and $l$ are parallel, and less attractive when $s$ and $l$ are antiparallel.

\section{Spectral function and occupancy}

The nuclear spectral function $S(E, p)$ is defined as the probability of finding a nucleon with the energy $E$ and initial momentum $p$ inside a nucleus. It is not an experimental observable but can be extracted from $\mathrm{A}(\mathrm{e}, \mathrm{e}$ 'p) cross section measurements [12]. In 
Further Splitting due to spin-orbit effects States

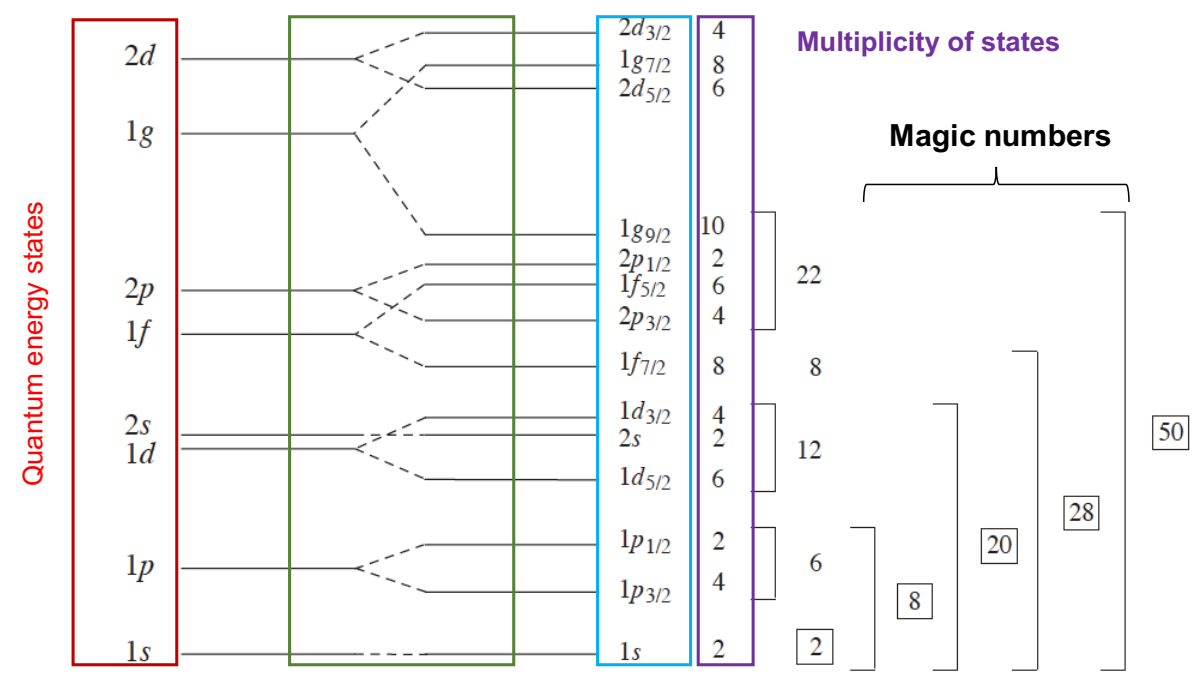

Figure 1.3: The magic numbers can be explained extremely well in the IPSM. The energy level for a given principle quantum number $n$ is split due to angular momentum effects and then further split due to the spin-orbit effects. Each energy state is defined by 3 quantum numbers $n, l, j$. The number of multiplicity for each states is predict by the IPSM and it reproduces nicely the magic numbers.

the IPSM, the spectral function can be expressed as:

$$
S(E, p)=\sum_{\alpha}\left|\Psi_{\alpha}(p)\right|^{2} \delta\left(E+\epsilon_{\alpha}\right),
$$

where $\Psi_{\alpha}$ and $\epsilon_{\alpha}$ are the single-particle wave functions and energies. Figure 1.4 shows the experimental spectral function for nucleus ${ }^{9} \mathrm{Be}[12]$.

The momentum distribution $n(p)$ can be obtained by integrating the spectral function over energy:

$$
n(p)=\int S(E, p) \cdot d E
$$

In the IPSM, $N^{o c c}$ is occupancy of a shell, determined by energy, can be predicted using the spectral function:

$$
N^{o c c}=4 \pi \iint^{k_{f}} p^{2} \cdot S(E, p) \cdot d p \cdot d E .
$$




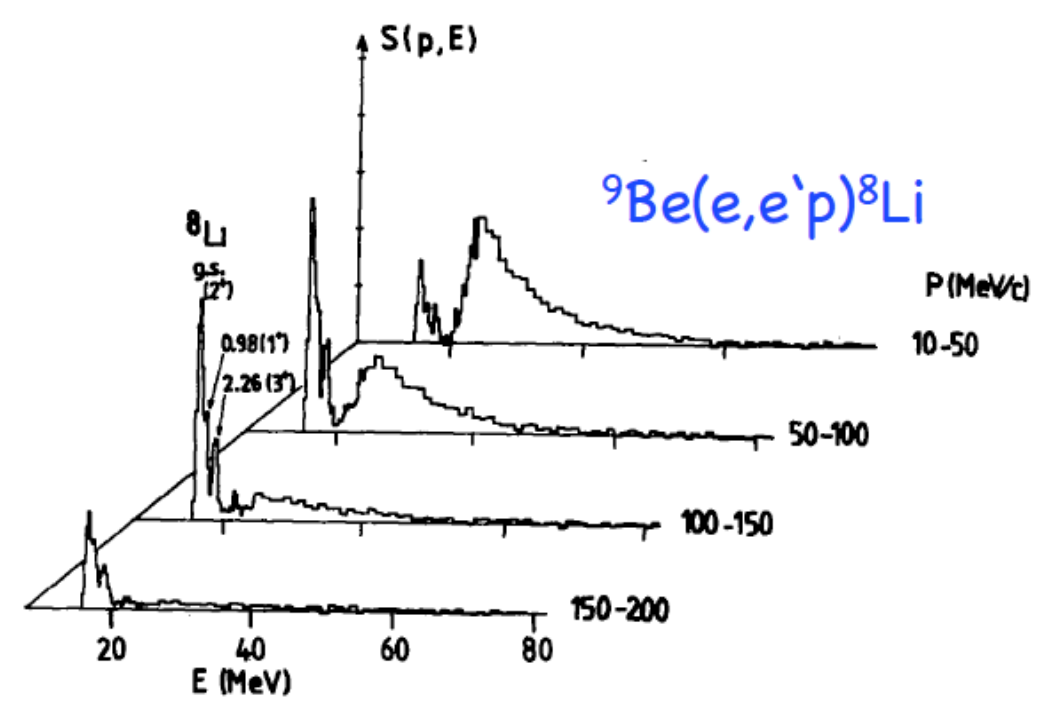

Figure 1.4: The Be spectral function as a function of separation energy $E$ for various momentum ranges [12] where several peaks in energy can be seen. These peaks are identified by knocked out nucleons from different ground state orbitals.

In experiment, the occupancy number can be determined by counting the number of knocked out protons from a given shell through the A(e,e'p) reaction. The comparison between the prediction and the data can be used to validate the model.

The IPSM is not only successful in describing the shell structure of nucleus, it is also successful to predict properties of nuclei such as spin, parities, magnetic dipole momentum [5, 8]. However, the IPSM also has its limitations due to the mean-field approximation. The IPSM's limitations and possible solution for those are presented below.

\subsection{Indirect evidence of SRCs}

The IPSM has been the standard model for nuclear physics since the 1950's. It motivated a lot of experiments to test the model, and many of them proved its success. Since the early 1970s, experimental data started showing evidence of the IPSM's limitation by observation of a clear discrepancy between the data to the model 
predictions. . In the 1980's a comprehensive program of $A\left(e, e^{\prime} p\right)$ experiments [7], detecting the knock-out proton along with scattered electrons, were performed at NIKHEF. Figure 1.5 shows a diagram of $A\left(e, e^{\prime} p\right)$ reaction where initial electron of energy and momentum $\left(E_{0}, \mathbf{k}\right)$ interacts with nucleus $A$ through exchange a virtual photon of energy and momentum $(\nu, \mathbf{q})$. The scattered electron $e^{\prime}$ carries the energy and momentum $\left(E, \mathbf{k}^{\prime}\right)$. The transfered energy is given as $\nu=E_{0}-E$. The missing momentum is $\mathbf{p}_{\text {miss }}=\mathbf{q}-\mathbf{p}$ with $\mathbf{p}$ the scattered nucleon momentum and $E_{m}=$ $\nu-T_{p}-T_{A-1}$ is the missing energy with $T_{p}$ and $T_{A-1}$ the kinetic energy of the knock-out proton and the recoiling nucleus, respectively.

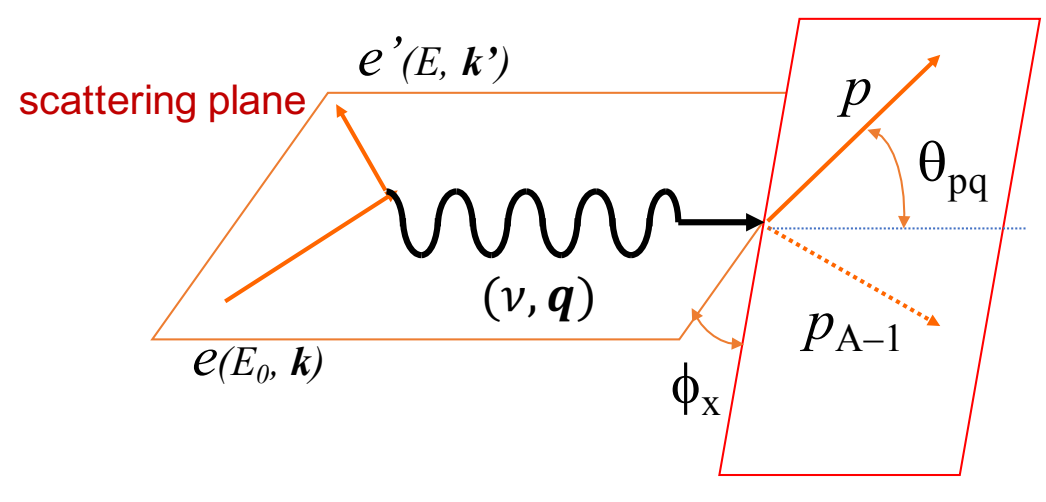

reaction plane

Figure 1.5: The electron scattering for $A\left(e, e^{\prime} p\right)$ reaction.

The experiments at NIKHEF were designed to focus on the single-particle region, small missing energy $\left(E_{m}<30 \mathrm{MeV}\right)$ and moderate missing momentum $\left(p_{m}<\right.$ $250 \mathrm{MeV})$. The $A\left(e, e^{\prime} p\right)$ reaction can be treated in the plane wave impulse approximation (PWIA) in which it is assumed that the virtual photon only interacts with a single nucleon. It is also assumed that there is no interaction between the struck nucleon and the recoiling nucleus. The cross section measured for each orbit in different nuclei then is used to extract the momentum distribution. The extracted momentum distribution can be compared to the prediction from the IPSM. In these knock-out proton measurements, outgoing protons experience a strong interaction 
with the residual nucleus which distorted the momentum distribution. To be able to compare to the experimental data, the DWIA (Distorted Wave Impulse Approximation) is applied on the effective potential of the IPSM for theoretical predictions [7]. The comparison is shown in Figure 1.6. Each plot displays the extracted momentum distribution from valence shell (upper data) and the next deeper shell (lower data) for different nuclei (each shell indicated by quantum numbers $n, l, j$ ). The solid curves present the results using DWIA. Normalization factors are used to scale the curve to the data. The shape of the momentum distributions from theory and data agree together almost perfectly but not in absolute strength.

Another result from this program shows that the extracted spectroscopic factor, which is the ratio of the observed strength of a given shell to the predicted strength, is less than 1 for the valence shell (close to the Fermi edge) (see Figure 1.7). For

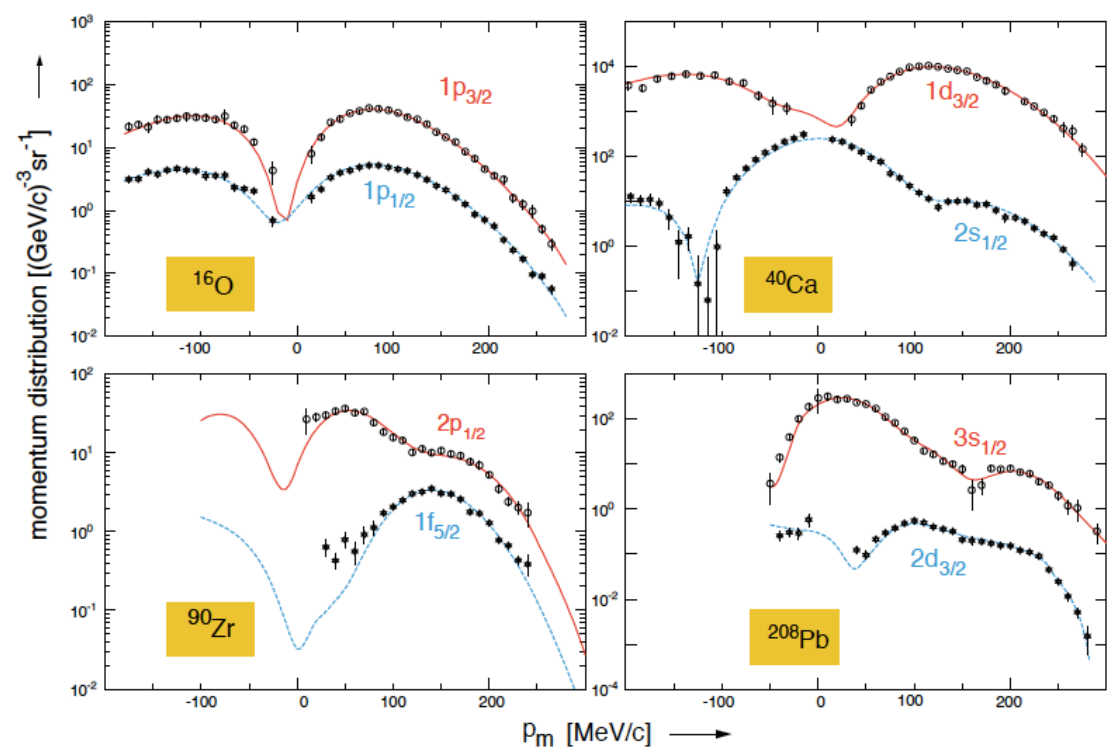

Figure 1.6: The momentum distribution results from the $A\left(e, e^{\prime} p\right)$ experiments at NIKHEF [7, 13] for different nuclei and quantum states as indicated (data points) and compared to the calculation from DWIA (solid curves). In each plot, the upper data is for the valence shell and the lower data is a for the next deeper shell. Normalization factors were used to scale the theory curves.

nuclei with $A>7$, this spectroscopic factor is almost a constant, $\sim 65 \%$, over a large 

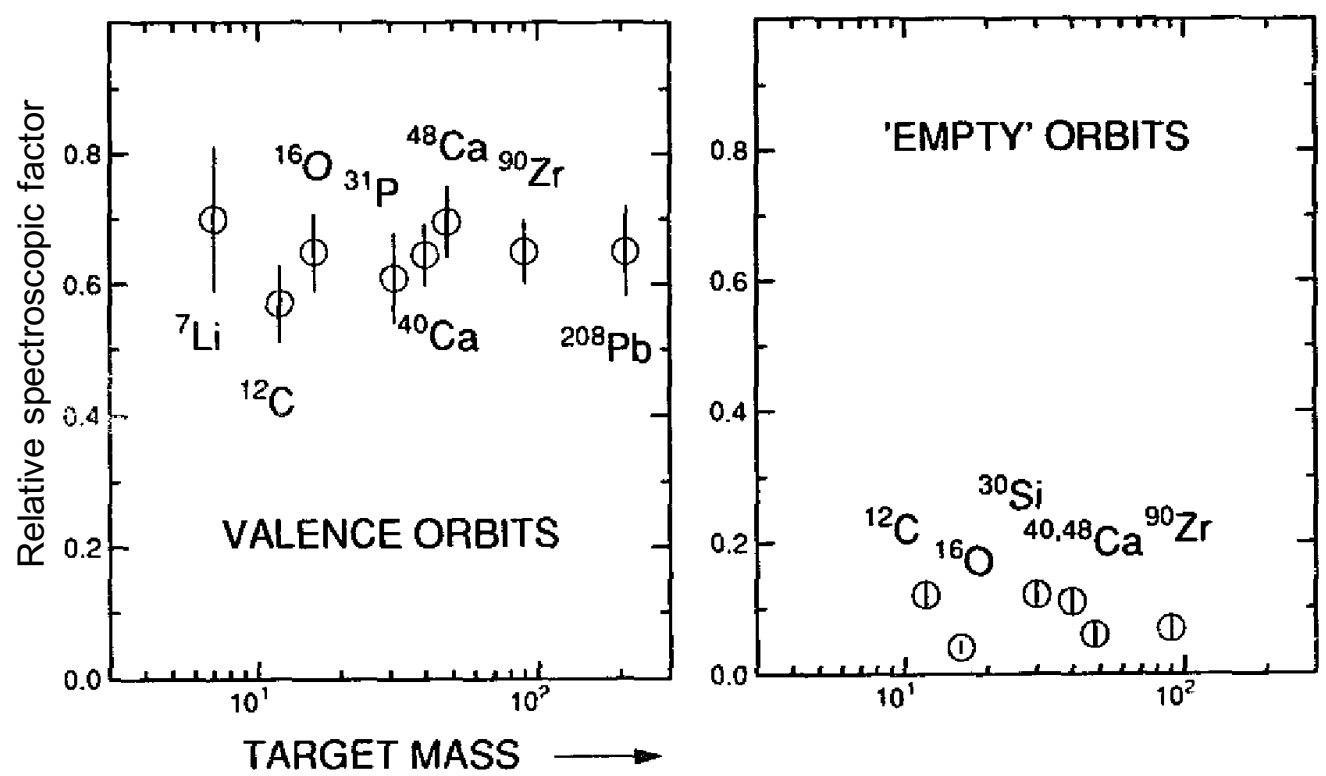

Figure 1.7: Left plot shows the spectroscopic factor for valance shell from $A\left(e, e^{\prime} p\right)$ experiment at NIKHEF as a function of target mass. For nuclei $\mathrm{A}>7$ this spectroscopic factor is almost a constant $\sim 65 \%$. Right plot shows a small spectroscopic factor for the shell above but close to valance shell [7].

range of $\mathrm{A}$. When the two body correlations at the distance of several fermi, longrange correlations (LRCs), were included in the calculation, there is still a significant discrepancy from the observed strength to the predicted strength from the IPSM [14]. This is a clear indication that the IPSM overestimates the occupancy of the valence shells. In addition, the experimental data also observed nucleons in the shells above but close to the Fermi edge [7] which is also not allowed in the IPSM. A possible explanation for this significant discrepancy is the presence of two-body short range correlations in nuclei, not included in the IPSM.

The observation of the difference in spectroscopic factors is considered as an indirect evidence of short range correlations. In the following sections, the features of the SRCs are discussed in more detail which helps to provide a better understanding of the limitations of the IPSM. 


\subsection{The Origin and Features of SRCs}

\section{Nucleon-Nucleon potential}

As discussed above, the IPSM is extremely successful explaining the shape of the momentums distribution for valence orbitals, not their absolute normalization. The IPSM is a single-body approximation, so a natural approach is to account for the two body correlations, particularly the short range correlations (SRCs). These correlations depend directly on the N-N potential. There are several realistic N-N potentials extracted from proton-proton and proton-neutron scattering data. The AV18 [15], Bonn [16 and Reid93 [17] N-N potentials are shown in the left hand side of Figure $1.8[18]$. The right plot shows two individual components (the tensor part and central part) and the total N-N potential from AV18 for nucleons in the deuteron [19]. A strong repulsive potential at very short distances is a common feature of all N-N potentials. The central component of the N-N potential is the dominant interaction out to a distance of $\sim 0.7 \mathrm{fm}$, where it makes a transition from the attractive to the repulsive, after that the tensor component is the dominant interaction. In addition, without this tensor component there would be no bound nuclei, since the attractive strength from the central part is not strong enough. This is the hint of the SRCs must be deuteron-like in the nucleus.

\section{Momentum distribution}

The strong repulsive core and the tensor part the of $\mathrm{N}-\mathrm{N}$ potential create a strong interaction between nucleons when they are close together, forming SRCs pairs of nucleons in the nucleus. These SRCs pairs have small total momentum (center-of mass momentum) but high relative momentum (above the Fermi momentum). In other words, these short range correlations can be understood in the way that they move strength away from the bound-states into the continuum energy and momen- 

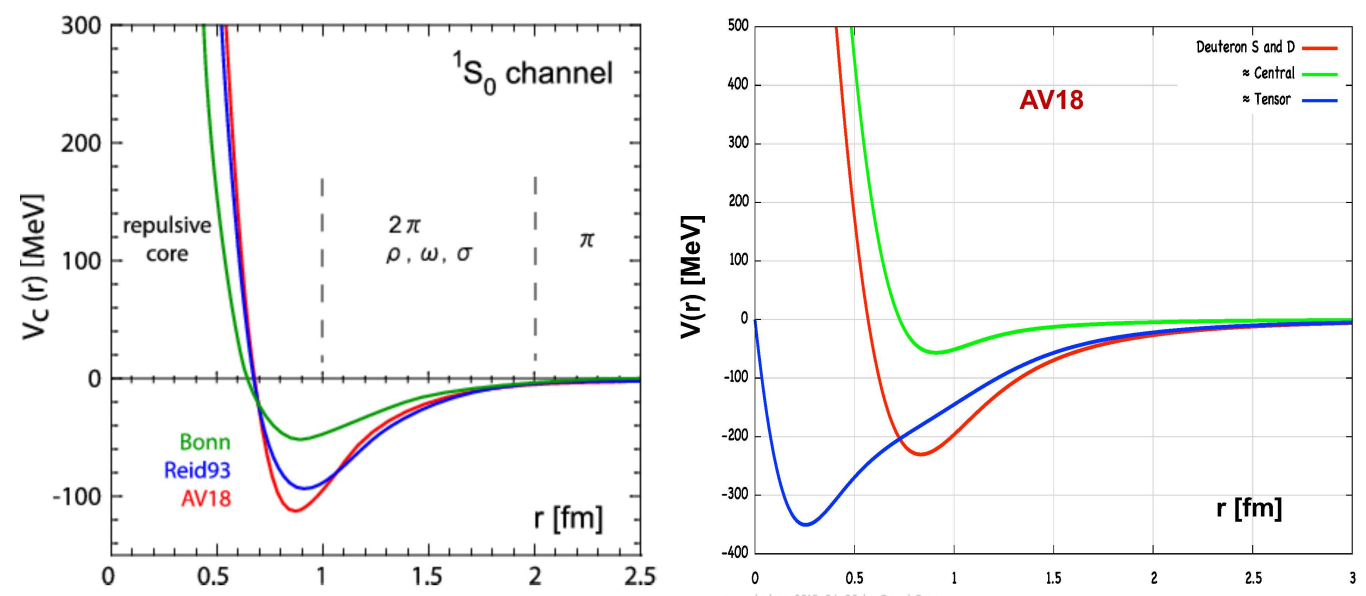

Figure 1.8: Left plot [18] shows the central component of the N-N potential from different groups, indicated in the plots. (Right): two individual components (central and tensor) and the total N-N potential from the AV18 group [19].

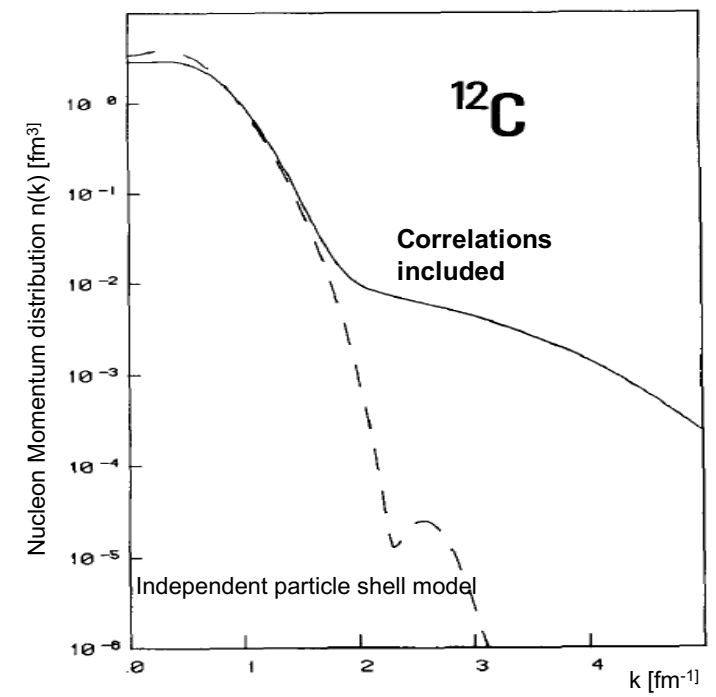

Figure 1.9: ${ }^{12} \mathrm{C}$ momentum distribution comparison, the dashed line and solid line present the mean-field momentum distribution and the one including the SRC correlations in the calculation, respectively [20].

tum states which has no strength in the IPSM [14,20,22]. This can be seen clearly in the comparison of mean-field momentum distributions and the realistic momentum distributions which include the correlations in their calculation (see Figure 1.9] [20]. The ${ }^{12} \mathrm{C}$ mean-field momentum distribution drops rapidly when the momentum ap- 

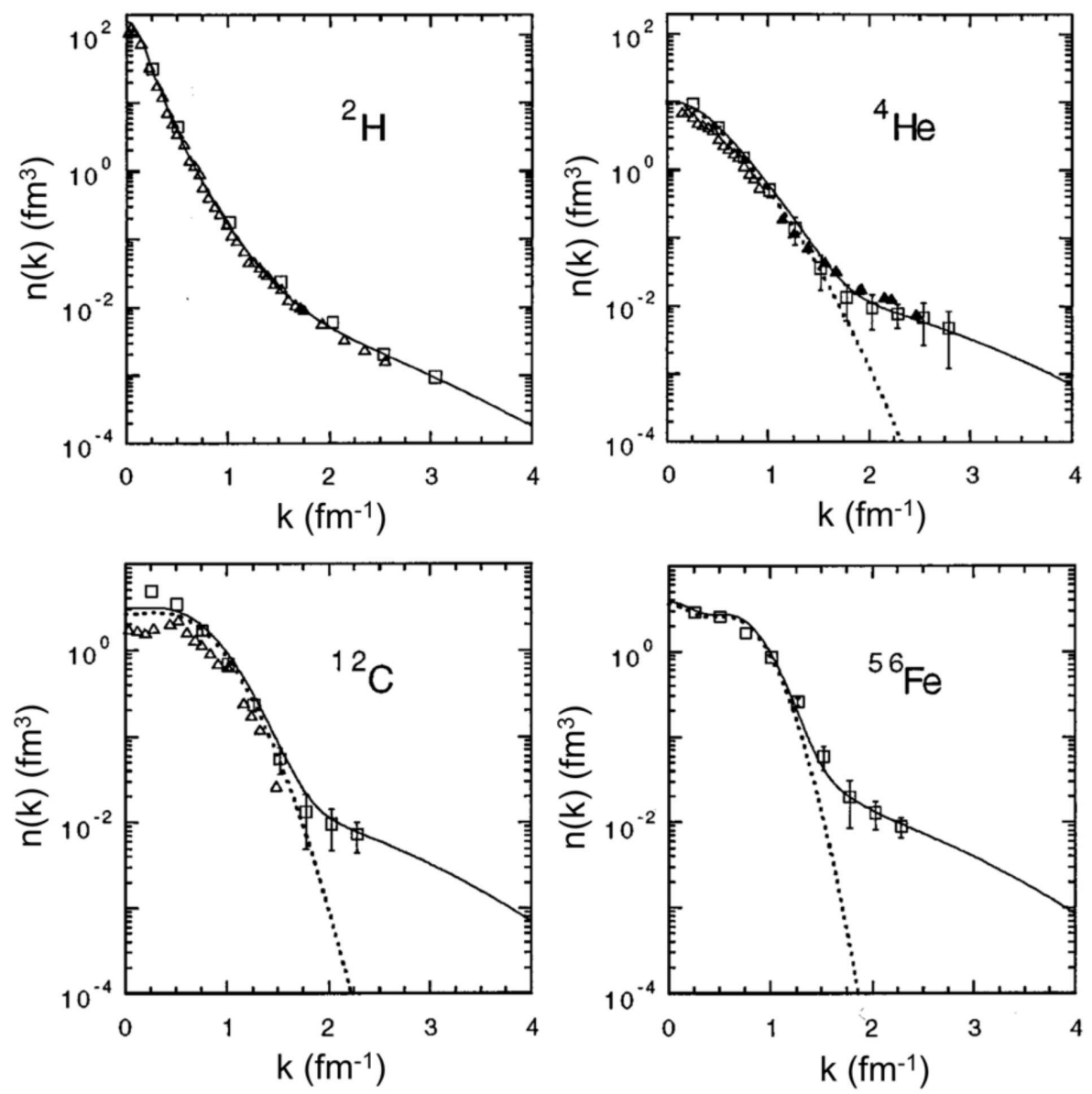

Figure 1.10: Comparison of mean-field momentum distributions (dashed line), a realistic momentum distributions (solid line) which include the SRC correlations to experimental data (symbols) [21].

proaches the Fermi momentum while the realistic distribution drops much slower and has a smooth transition to a high momentum tail. The comparison also clearly shows that the momentum distribution at low momentum is reduced and enhanced at high momentum by including the correlations. In addition, the experimental data (see Figure 1.10 show a very good agreement with realistic momentum distribution [21.

Under the assumption that short range correlations are responsible for the high momentum tail the experimental data is reproduced very well which was not possible within the IPSM. This assumption also suggests that the high momentum tails in different nuclei have a common source, have the same shape, and only differ by a 
scaling factor. This scaling factor depends on the probability of finding SRC pairs in different nuclei. The momentum distribution at large $k$ for different nuclei can be scaled from ${ }^{2} \mathrm{D}[21]$. It can be expressed as:

$$
n_{A}(k)=a_{2}(A) \cdot n_{D}(k)
$$

where $n_{D}(k)$ and $n_{A}(k)$ are the ${ }^{2} \mathrm{D}$ and nucleus (with atomic number $\mathrm{A}$ ) momentum distribution and $a_{2}(A)$ is the corresponding scaling factor.

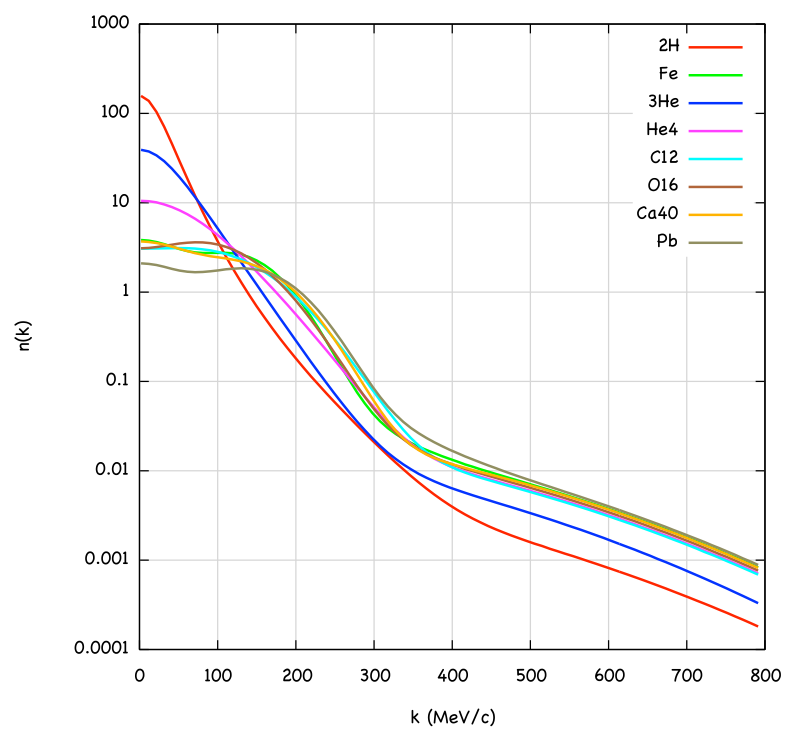

Figure 1.11: The momentum distribution for different nuclei, scaled from the ${ }^{2} \mathrm{D}$ momentum distribution using different scaling factors 21 .

There are many reactions that can be used to explore SRCs. Inclusive electron scattering is the simplest one and will be discussed in the next section.

\subsection{Inclusive Electron Scattering}

Inclusive electron scattering from nuclei $A\left(e, e^{\prime}\right)$, is a process where an electron $e$ scatters off a nucleus $A$ and only the scattered electron $e^{\prime}$ is detected. This reaction gives the opportunity to study different properties of nuclear matter depending on the 
kinematic region. Figure 1.12 shows the quasi-elastic diagram of the $A\left(e, e^{\prime}\right)$ reaction.

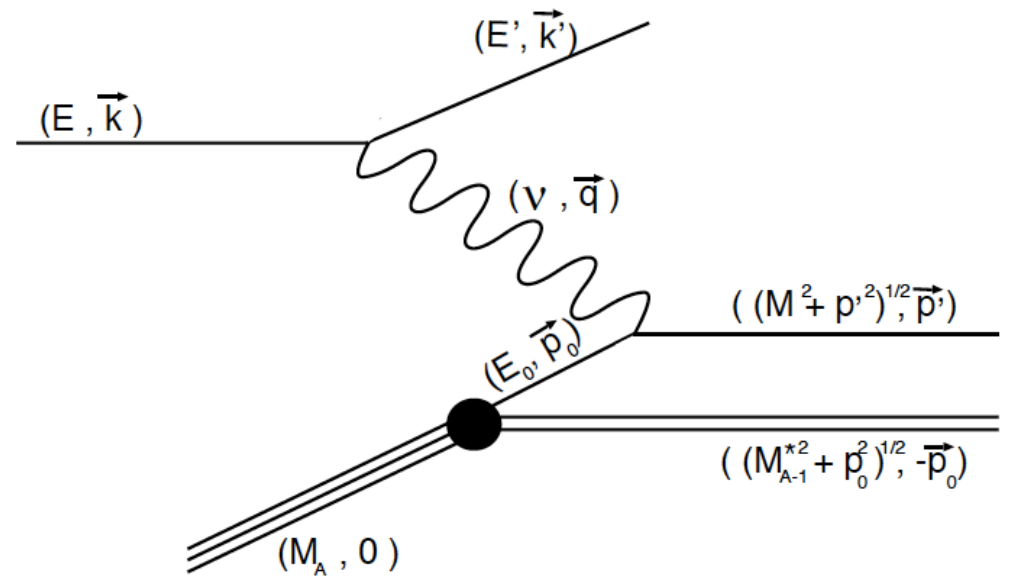

Figure 1.12: Electron-nucleus quasi-elastic scattering diagram for $A\left(e, e^{\prime}\right)$ reaction taken from Ref. [23]. $\mathrm{M}_{A-1}^{*}$ is the mass of recoiling $(A-1)$ system.

\section{Kinematic variables}

The electron with energy and momentum $\left(E_{0}, \vec{k}\right)$ interacts with the nucleus $A$ through exchange of a virtual photon of energy and momentum $(\nu, \vec{q})$. The scattered electron $e^{\prime}$ carries the energy and momentum $\left(E, \overrightarrow{k^{\prime}}\right)$. The transfer energy is $\nu=E_{0}-E$. The invariant mass of the undetected final hadronic state, $W^{2}$, and 4-momentum transfer, $Q^{2}$ are defined as:

$$
Q^{2}=\vec{q}^{2}-\nu^{2} \quad \text { and } \quad W^{2}=2 m_{p} \nu+m_{p}^{2}-Q^{2} .
$$

where $m_{p}$ is the proton mass. The most commonly used kinematic variable in SRCs studies is Bjorken $x_{b j}=\frac{Q^{2}}{2 m_{p} \nu}$. There is another common kinematic variable $y$, which is defined later. $Q^{2}$ and $x_{b j}$ are used as a tuning knob to access the different kinematic regions (see Figure 1.13). The spectrum of inclusive electron-nucleus cross section is shown as a function of transferred energy $\nu$ in Figure 1.13. Different kinematic regions are sensitive to different physics. Elastic scattering is used to study the 
structure of the nucleon or nucleus: the form factor and charge distribution. Deep Inelastic Scattering (DIS) is used to study quark distribution functions, and possibly, their modification inside the nucleus. Quasi-elastic scattering can be used to study momentum distributions and nucleon-nucleon SRCs.

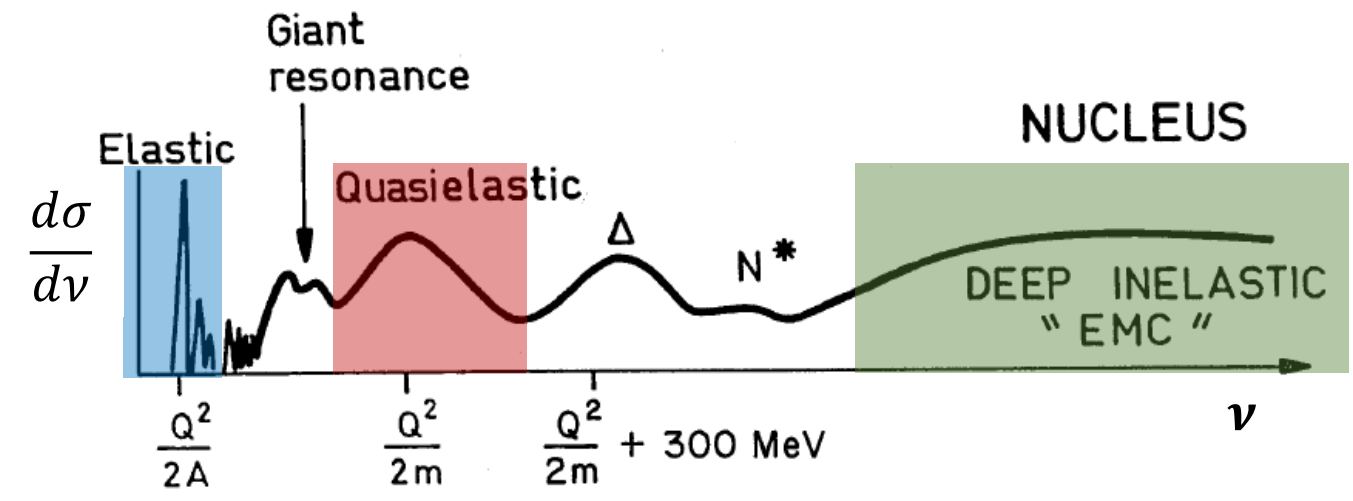

Figure 1.13: The inclusive cross section for electron scattering off a nucleus as a function of transfer energy $\nu$.

\section{Scaling functions}

Scaling is a term used to describe a cross-section, a function of 2 independent variables, which under some conditions can be described as a function of a single variable itself derived from the same variables, here $Q^{2}$ and $\nu$. In inclusive scattering the scaling function is a very useful way to study a complex system, because the observation of scaling indicates a simple mechanism or substructure in the system which can be examined using the scaling function itself. In addition, the violation of the expected scaling behavior can be used to validate the assumptions in the model that predicts scaling. For different kinematical regions, there are different scaling functions. In the following sections we will discuss the quasi-elastic scattering and its scaling function, $F(y)$. 


\subsubsection{Quasi-elastic scattering}

Quasi-elastic scattering is defined as a process where a single nucleon is knocked out from the nucleus by an electron, with the recoiling $(A-1)$ left in any state, see Figure 1.12. In electron-nucleus scattering quasi-elastic is the dominant process in the kinematic region with the energy transfer $0.5 \mathrm{GeV}<\nu<2 \mathrm{GeV}$. The contribution from inelastic scattering increases with $Q^{2}[24,25]$. The quasi-elastic peak is located at $x_{b j}=1$ but broadened due to the motion of the nucleons inside the nucleus. By assuming the quasi-elastic model for electron scattering off a single nucleon within the nucleus to be valid, the peak region can be described in terms of a scaling function $F(y)$ which will be derived in the next section.

\section{Quasi-elastic cross section}

The quasi-elastic cross section can be calculated using the plane wave impulse approximation (PWIA) 23, 26]. Two main assumptions are used in this approximation: no final-state interaction between the knock-out nucleon and the recoiling nucleus, and the photon interacts only with a single moving nucleon. The inclusive cross section can be obtained using the expression [26, 27]:

$$
\begin{aligned}
\frac{d^{2} \sigma}{d E_{e^{\prime}} d \Omega_{e^{\prime}}} & =\sum_{i=1}^{A} \int d \vec{p}_{0} \int d E_{s} \cdot \sigma_{e i} \cdot S_{i}\left(p_{0}, E_{s}\right) \\
& \times \delta\left(\nu+M_{A}-\sqrt{M^{2}+p^{\prime 2}}-\sqrt{M_{A-1}^{* 2}+p_{0}^{2}}\right)
\end{aligned}
$$

where $\vec{p}_{0}$ and $\overrightarrow{p^{\prime}}=\vec{p}_{0}+\vec{q}$ are the initial and the final momentum of the knock-out nucleon, $E_{s}=M_{A-1}^{*}+M-M_{A}$ is the separation energy. $S_{i}\left(p_{0}, E_{s}\right)$ is the spectral function of the nucleus ( $i$ stands for either the proton or the neutron) and $\sigma_{e i}$ is the electron-nucleon cross section for scattering from a bound moving nucleon. $M_{A}$ and $M_{A-1}^{*}$ are the nucleus mass and the residual $(A-1)$ system mass, respectively. The argument of the $\delta$ function is for energy and momentum conservation. The 
spectral function is spherically symmetric so $d \vec{p}_{0}=p_{0}^{2} \cdot d p_{0} d(\cos \vartheta) d \varphi$, where $\vartheta$ is angle between $\vec{q}$ and $\vec{p}_{0}$ and $\varphi$ is the angle between electron scattering plane and the reaction plane. The difference between the spectral function for protons and neutrons is usually neglected. Summing over $A$ nucleons and integrating over the angle $\vartheta$, the cross section can be rewritten as:

$$
\frac{d^{2} \sigma}{d E_{e^{\prime}} d \Omega_{e^{\prime}}}=2 \pi \int_{E_{s}^{\min }}^{E_{s}^{\max }} \int_{p_{0}^{\min }\left(E_{s}\right)}^{p_{0}^{\max }\left(E_{s}\right.} \overline{\sigma_{0}} \cdot S\left(p_{0}, E_{s}\right) \cdot p_{0} \cdot d p_{0} \cdot d E_{s}
$$

where $\overline{\sigma_{0}}=\sigma_{0} \cdot \frac{\sqrt{M^{2}+{\overrightarrow{p^{\prime}}}^{2}}}{q}$ and $\sigma_{0}$ can be expressed as:

$$
\sigma_{0}=\frac{1}{2 \pi} \sum_{i=1}^{A} \int_{0}^{2 \pi} \sigma_{e i} d \varphi=\frac{1}{2 \pi} \int_{0}^{2 \pi}\left(Z \sigma_{e p}+N \sigma_{e n}\right) d \varphi
$$

The energy limit $E_{s}^{\text {min }}$ corresponds to the $A-1$ system being in its ground state while $E_{e}^{\text {max }}$ corresponds to the struct nucleon being at rest.

$$
E_{s}^{\text {min }}=M_{A-1}+M-M_{A} \quad \text { and } \quad E_{s}^{\max }=\sqrt{\left(\nu+M_{A}\right)^{2}-q^{2}}-M_{A}
$$

The limits of the momentum integration $p_{0}^{\min }$ and $p_{0}^{\max }$ are determined by the constraint $-1 \leq \cos \vartheta \leq 1$, i.e $\vec{p}_{0}$ is parallel to $\vec{q}$.

\section{Scaling function $F(y)$}

The $y$ variable can be obtained from 26

$$
\nu+M_{A}=\sqrt{M^{2}+q^{2}+y^{2}+2 y q}+\sqrt{M_{A-1}^{2}+y^{2}}
$$

The cross section $\overline{\sigma_{0}}$ in Equation 1.10 varies extremely slowly with $p_{0}$ and $E_{s}$ while the spectral function changes rapidly. This means that it is a good approximation to replace $\overline{\sigma_{0}}\left(p_{0}, E_{s}\right)$ by its value at the peak of the spectral function $\tilde{\sigma}=\overline{\sigma_{0}}\left(p_{0}^{\min }, E_{s}^{\min }\right)$. 
The integration limits can be extended to infinity. The rapid decrease of the spectral function means that the error by extending the integration limits to infinity will decrease rapidly as $Q^{2}$ increases (see Ref. [23]). The cross section can be written as:

$$
\frac{d^{2} \sigma}{d E_{e^{\prime}} d \Omega_{e^{\prime}}}=2 \pi \tilde{\sigma} \int_{E_{s}^{\min }}^{\infty} \int_{|y|}^{\infty} S\left(p_{0}, E_{s}\right) \cdot p_{0} \cdot d p_{0} \cdot d E_{s}
$$

where $|y|=p_{0}^{\min }\left(E_{s}^{\min }\right)$. The scaling function $F(y)$ is defined as :

$$
F(y)=2 \pi \int_{E_{s}^{\min }}^{\infty} \int_{|y|}^{\infty} S\left(p_{0}, E_{s}\right) \cdot p_{0} \cdot d p_{0} \cdot d E_{s} .
$$

The momentum distribution of a nucleon inside the nucleus $n\left(p_{0}\right)$ is the energy integral over the spectral function.

$$
n\left(p_{0}\right)=\int_{E_{s}^{\min }}^{\infty} S\left(p_{0}, E_{s}\right) \cdot d E_{s}
$$

The scaling function $F(y)$ can be rewriten as:

$$
F(y)=2 \pi \int_{|y|}^{\infty} n\left(p_{0}\right) \cdot p_{0} d p_{0}
$$

The momentum distribution can be calculated using the $F(y)$ using experession [28:

$$
n\left(p_{0}\right)=-\frac{1}{2 \pi p_{0}} \cdot \frac{d F(y)}{d y}
$$

By using the scaling function $F(y)$, the cross section is separated into two terms (see Equation 1.12). The first term, $\tilde{\sigma}$, represents the interaction process. The second term, $F(y)$, carries the structure of the nucleus, related directly to the momentum distribution of the strucked nucleon. The $F(y)$ scaling function can be extracted from 
experimental data using the description:

$$
F(y)=\frac{d^{2} \sigma}{d E_{e^{\prime}} d \Omega_{e^{\prime}}} \cdot \frac{1}{Z \sigma_{e p}+N \sigma_{e n}} \cdot \frac{q}{\sqrt{M^{2}+\left(\vec{p}_{0}+\vec{q}\right)^{2}}}
$$

$\sigma_{e p}$ and $\sigma_{e n}$ are the off-shell elementary electron-proton and electron-neutron cross section [29]. In order to understand this scaling behavior, the extracted cross-section and $F(y)$ for ${ }^{3} \mathrm{He}$ from SLAC data are shown in Figure 1.14 30,31. The cross section for many different kinematic settings collapse into a single line, $F(y)$.
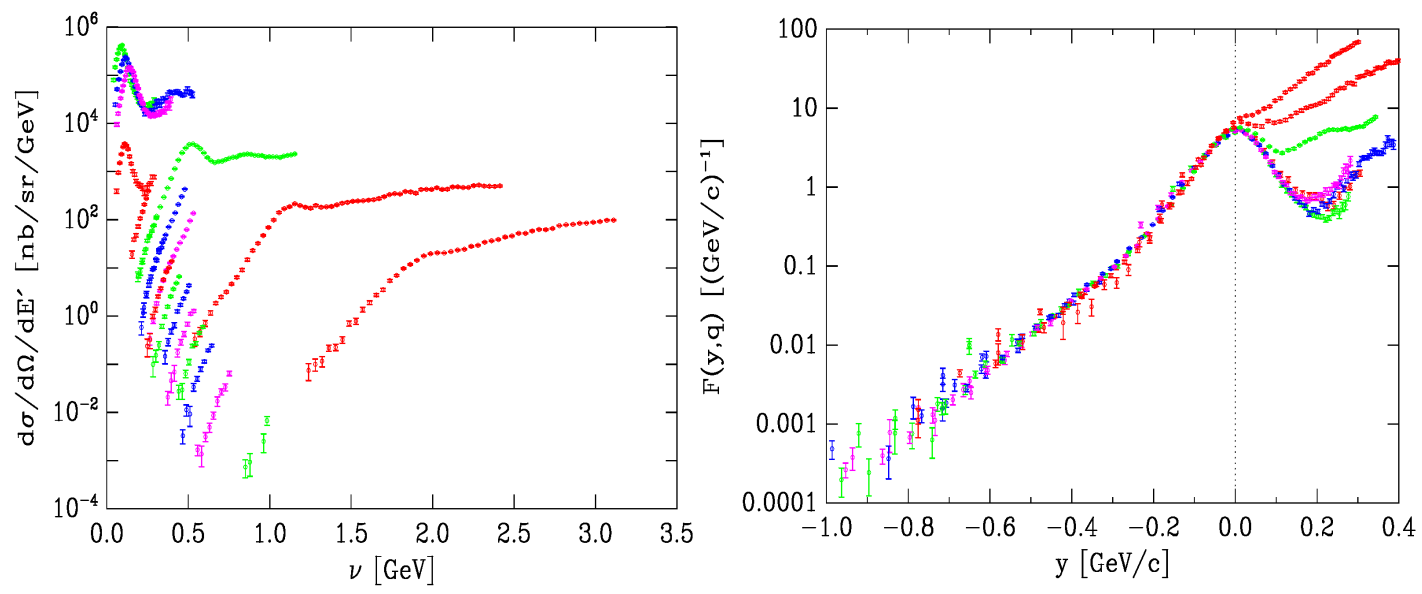

Figure 1.14: The experimental cross section (left) and $F(y)$ (right) for ${ }^{3} \mathrm{He}$ from SLAC data [30]. The plots courtesy of D. Day. The colored lines cover a range of data with $Q^{2}$ from $0.25 \mathrm{GeV}^{2}$ to $4 \mathrm{GeV}^{2}$.

$F(y)$ appears to be scale well in the region of $-0.6<y<0$ (independent of $Q^{2}$ ) which supports the underlying assumption of Quasi-elastic scattering. At low $Q^{2}$ the final state interactions increase would break the scaling. At high $Q^{2}$ and large energy transfer the contribution from inelastic data increases also breaks scaling.

\subsubsection{Where to find SRCs in Inclusive Scattering}

As discussed above, the SRCs are responsible for the high momentum tail of the nucleon momentum distribution. SRCs can be studied via inclusive electron scatter- 
ing by working in a special kinematic region where the reaction can only come from an interaction with a correlated high momentum nucleon which is instantaneously removed from the SRC. This requirement can be achieved if the energy and momentum transfer scales are much larger than scale characteristic for SRCs [32, 33]. This condition requires:

$$
\nu \gg V_{N N} \quad \text { and } \quad|\vec{q}| \gg 2 k_{F}
$$

where $V_{N N}$ is the characteristic potential of the $N-N$ interaction. Another background which needs to be suppressed is the contribution of long range interactions through meson exchange. This can be achieved by required $Q^{2} \gg m_{m e s o n}^{2}[34]$.

In order to fulfill the requirement that the minimal momentum of the struck nucleon, $P_{\min }$ must be greater than $k_{f}$, a calculation of $P_{\min }(y)$ as a function of $x_{b j}$ and $Q^{2}$ is shown in Figure 1.15 . To access the region where SRCs are dominant, the electrons have to scatter off the nucleon with $x_{b j}>1.5$ and $Q^{2}>1.5$. The condition of large $x_{b j}\left(x_{b j}>1\right)$ kinematically restricts the scattering from a single nucleon and therefore gives access to the multi-nucleon reaction. In addition, requiring that the $x_{b j}>1$ minimizes inelastic scattering contribution, providing much cleaner data (see Figure 1.16).

Final state interactions (FSI), a severe concern in SRC studies, fall rapidly as the energy and momentum transfer increase because of the decrease of the interaction time (see Ref. [23]). In the kinematical regime of SRCs studies, FSI are not small but it is considered mainly as the interaction between nucleons in the SRC pair and not between the struck nucleon and the residual nucleus. This residual FSI effect is largely cancelled in the ratio of the cross sections. More information about FSI in SRCs can be found in the Refs. [14, 24, 33.

It is worth mentioning that for two nucleon SRCs the specific calculation to determine the kinematic region can be easily performed, but for the $3 \mathrm{~N}$ or higher SRCs the calculations become much more complicated. The most recent calculation for $3 \mathrm{~N}-$ 

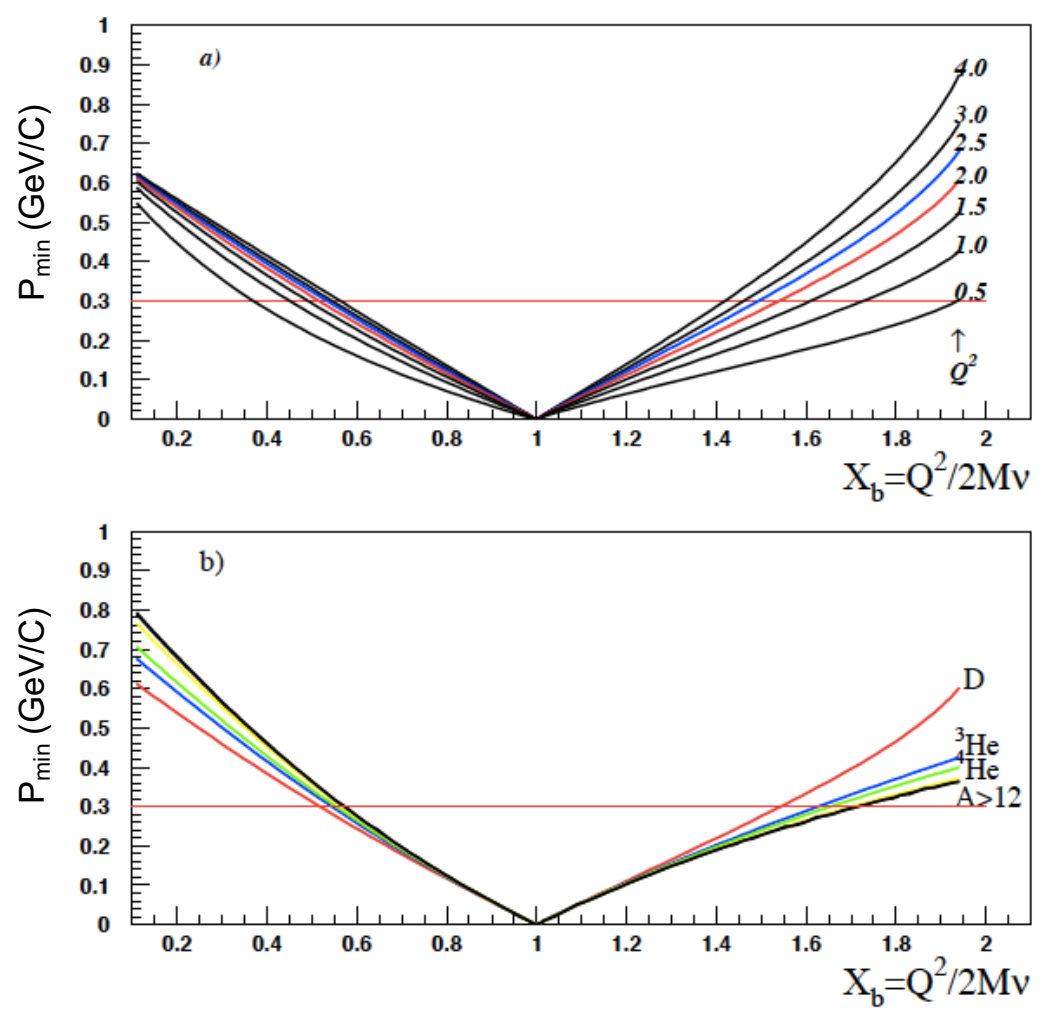

Figure 1.15: The minimum momentum of the struck nucleon is shown as a function of $x_{b j}$ and $Q^{2}$ for deuterium (Figure (a)) and for different nuclei (Figure (b)). The red line represents the Fermi momentum 33].

SRC kinematic limits was performed by M. Sargsian (see Figure 1.17] 35, 36. In this calculation the variable $\alpha_{3 N}$, a light cone variable associated with interacting nucleon belonging to $3 \mathrm{~N}-\mathrm{SRC}$, is used. The result of this calculation shows that $3 \mathrm{~N}-\mathrm{SRC}$ are dominant when $\alpha_{3 N}>1.6$. This means that if we want to observe $3 \mathrm{~N}$ SRCs in the region of $x_{b j}>2$, the minimum $Q^{2}=5 \mathrm{GeV}^{2}$ is needed.

In the kinematic region where $x_{b j}>1$ and $Q^{2}>1$ the SRCs are expected to be dominant, as discussed above, and the inclusive cross section can be approximated as:

$$
\begin{aligned}
\sigma_{A}\left(x, Q^{2}\right) & =\sum_{j=2}^{A} \frac{A}{j} a_{j}(A) \sigma_{j}\left(x, Q^{2}\right) \\
& =\frac{A}{2} \cdot a_{2}(A) \cdot \sigma_{2}\left(x, Q^{2}\right)+\frac{A}{3} \cdot a_{3}(A) \cdot \sigma_{3}\left(x, Q^{2}\right)+\ldots
\end{aligned}
$$




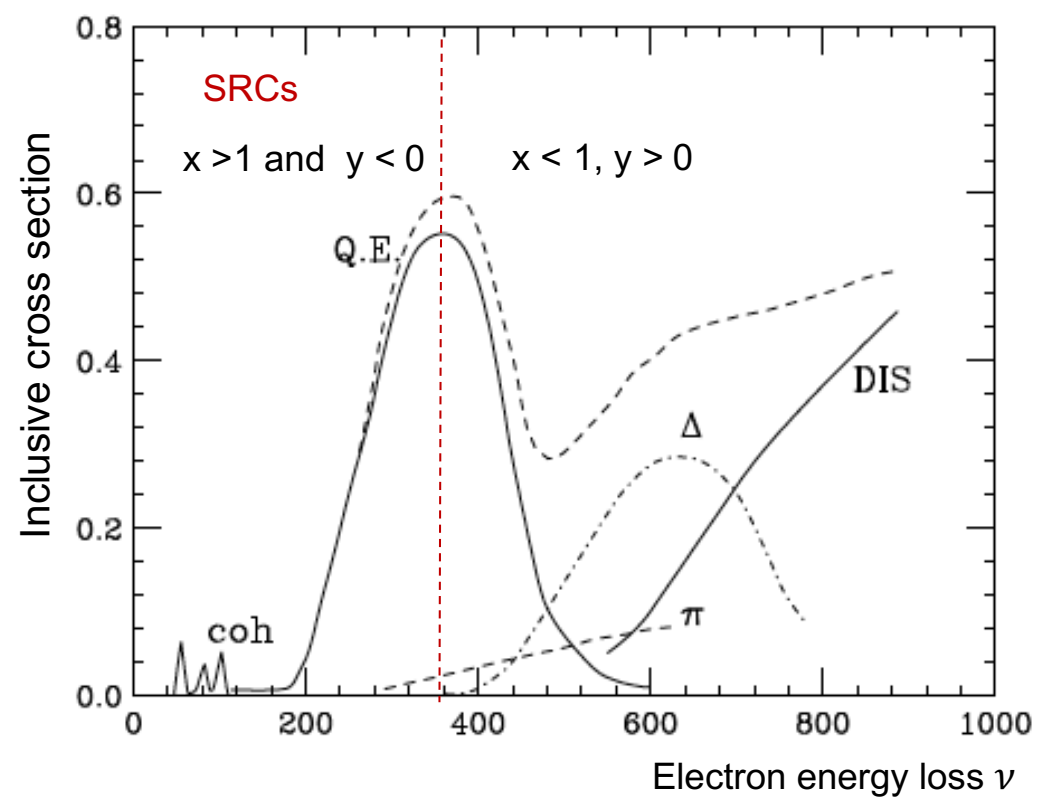

Figure 1.16: Schematic representation of inclusive cross section cross section [24. The total cross section as well as the individual contributions from Quasi-elastic, resonance, and DIS are presented in the plots as a function of $\nu$ in $\mathrm{MeV}$. The region for SRC studies is indicated in plot where the contribution from other reaction are suppressed.

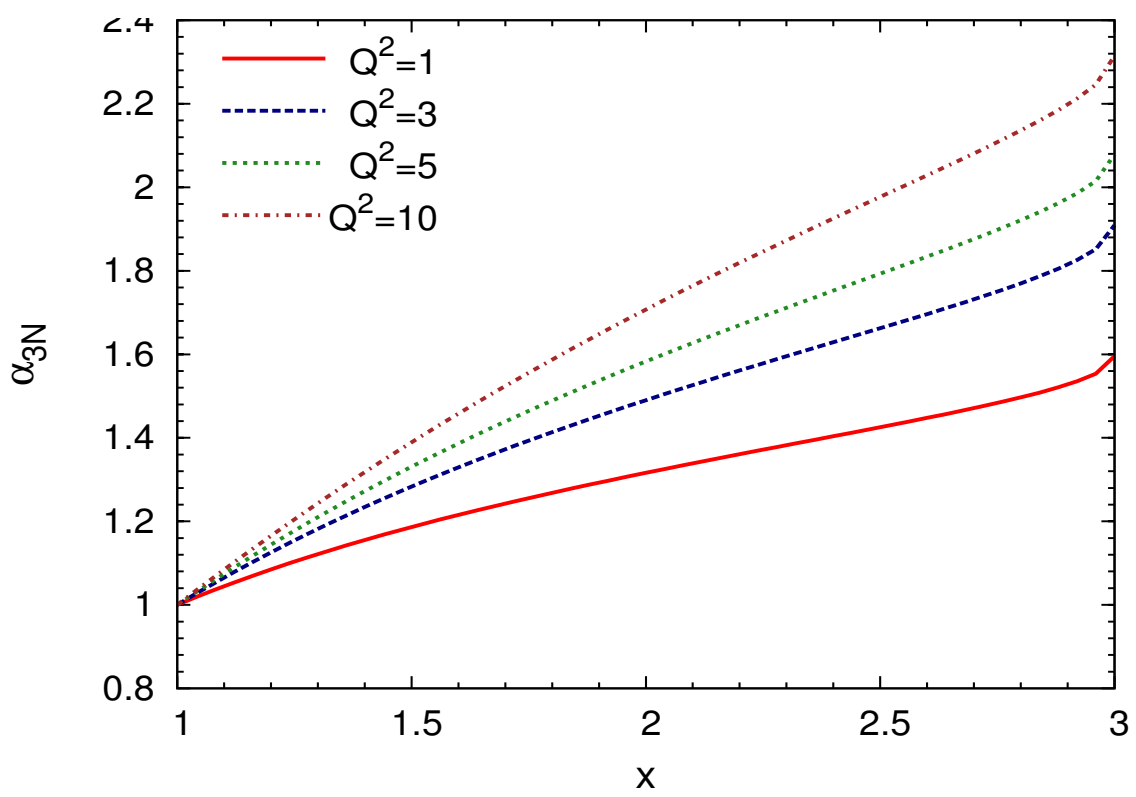

Figure 1.17: The $3 \mathrm{~N}$ SRCs kinematic calculation as a function of $x_{b j}$ and $\alpha_{3 N}$, a function of $x_{b j}$ and $Q^{2}$ [35]. 
The total cross section $\sigma_{A}\left(x, Q^{2}\right)$ is the sum of cross section from $j$-nucleon correlations, $\sigma_{j}\left(x, Q^{2}\right)$. The constant $a_{j}(A)$ is proportional to the probability of finding a nucleon in a $j$-nucleon correlation and decreases rapidly when $j$ is increasing. For the case, $A=j=2, a_{j}(A)=a_{2}(2)=1$. With this definition $a_{2}(A)$ is the probability of finding a $2 \mathrm{~N}-\mathrm{SRCs}$ in a nucleus $A$ relative to ${ }^{2} \mathrm{D}$. Another approximation is $\sigma_{j}\left(x, Q^{2}\right) \sim 0$ for $x>j$. This suggests that the 2N-SRCs are dominant in the region $1<x<2$. For $x>2$, the static $2 \mathrm{~N}-\mathrm{SRC}$ contribution vanishes (the motion of the $2 \mathrm{~N}-\mathrm{SRC}$ in nuclear can broaden it a bit above $x=2$ ) and the scattering is dominated by contributions of 3N-SRCs or higher. As discussed in Section 1.4, the high momentum tail of nucleon momentum distribution from different nuclei have the same shape and they differ only from each other by a scaling factor since they all come from a common origin, the short range part of $\mathrm{N}-\mathrm{N}$ potential. Therefore the cross section ratio should be a constant in the kinematics where 2N-SRCs are dominant. The cross section ratio from different nuclei to ${ }^{2} \mathrm{D}$ can be described by:

$$
\frac{2}{A} \sigma_{A}\left(x, Q^{2}\right) / \sigma_{D}\left(x, Q^{2}\right)=\frac{a_{2}(A) \cdot \sigma_{2}\left(x, Q^{2}\right)}{\sigma_{2}\left(x, Q^{2}\right)}=a_{2}(A),
$$

where the fraction $\frac{2}{A}$ make this cross section ratio per nucleon. If SRCs dominate we would expect to see in the per nucleon cross section ratios a plateaus for $1.5<x_{b j}<2$ and the height of the plateaus could provide $a_{2}(A)$. It is a natural choice to move from $2 \mathrm{~N}$ to $3 \mathrm{~N}-\mathrm{SRC}$ by using the ratio of cross section from heavy nuclei to ${ }^{3} \mathrm{He}$ in the region where $3 \mathrm{~N}-\mathrm{SRCs}$ are dominant. The cross section ratio can be expressed as:

$$
\frac{3}{A} \frac{\sigma_{A}\left(x, Q^{2}\right)}{\sigma_{3}\left(x, Q^{2}\right)}=\left.a_{3}(A)\right|_{2<x<3},
$$

where $a_{3}(A)$ is the probability of finding $3 \mathrm{~N}$ nucleons in nucleus $A$ relative to ${ }^{3} \mathrm{He}$. The results of experiments that have investigated SRCs in both $2 \mathrm{~N}$ and $3 \mathrm{~N}$ region are summarized in the next chapter. 


\section{Chapter 2}

\section{SRCs measurements}

Experiments have been performed to investigate SRCs at different labs, SLAC (Stanford Linear Accelerator Center), BNL (Brookhaven National Laboratory) and JLab (Jefferson Lab). These experiments used either electron or proton scattering in inclusive or exclusive reactions, $A\left(e, e^{\prime}\right), A\left(e, e^{\prime} p\right), A(p, p n)$ etc. Many important results were accumulated and have provided a much better understanding of SRCs. A summary and discussion of these results is presented in this chapter, followed by the motivation for E08014 and an outlook for future SRCs studies.

\section{$2.1 \quad$ Results of Inclusive experiments}

\section{The first direct evidence of SRC}

As discussed in Section 1.5, electron inclusive scattering is the simplest reaction to study the SRCs. The results from the experiments performed at SLAC are considered as the first direct evidence of SRC [37]. This experiment collected data in the kinematic region, $x_{b j}>1$ and $Q^{2}>1$, where SRCs are expected to be dominant (see Section 1.5.2. . The cross sections included ${ }^{2} \mathrm{D},{ }^{3} \mathrm{He},{ }^{4} \mathrm{He},{ }^{12} \mathrm{C},{ }^{27} \mathrm{Al},{ }^{56} \mathrm{Fe}$ and ${ }^{197} \mathrm{Au}$

were used to calculate the cross section ratio $\frac{\sigma A}{\sigma D} \frac{2}{A}$. Some of the results can seen in 
Figure 2.1 37.
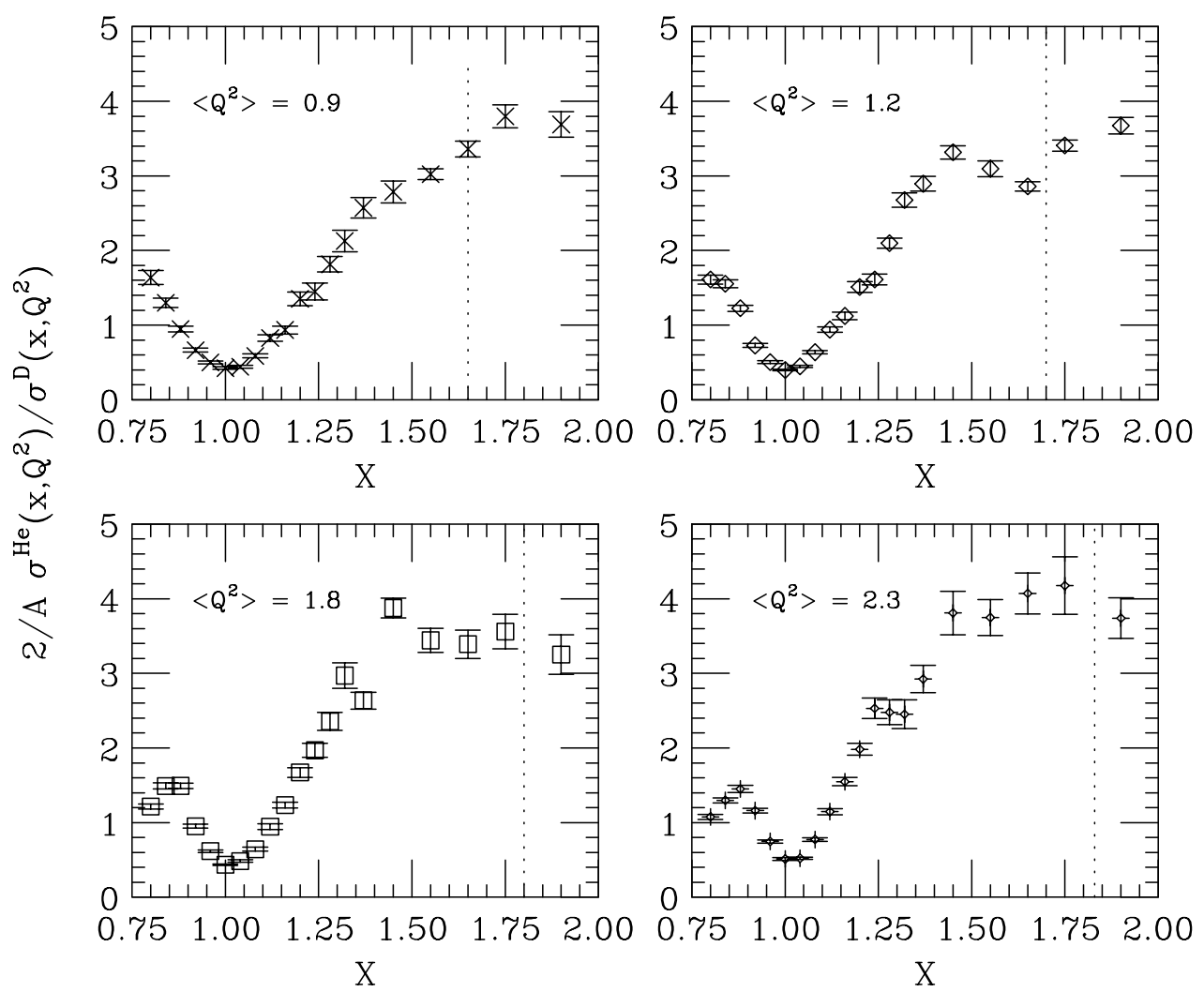

Figure 2.1: The first direct evidence of SRC from the SLAC data 37. The plots show the cross section ratio per nucleon of ${ }^{4} \mathrm{He}$ to ${ }^{2} \mathrm{D}$ as function of $x_{b j}$ for different $Q^{2}$ 's.

Figure 2.1 shows the cross section ratio from ${ }^{56} \mathrm{Fe}$ to ${ }^{2} \mathrm{D}$ as a function of $\mathrm{x}_{b j}$. Scaling (plateaus) was observed in the region $x_{b j}>1.5$ which indicates the existence of SRC. The plateaus also indicate the scattering took place from the high momentum nucleons (above $k_{F} \sim 250 \mathrm{MeV}$ ). The scaling factor $a_{2}(A)$ for different nuclei was obtained using the expression:

$$
a_{2}(A)=\frac{2 \cdot \sigma_{A}}{A \cdot \sigma_{D}}
$$

where $\sigma_{A}$ and $\sigma_{D}$ are the cross section from the nucleus $A$ and deuteron, respectively.

The results from SLAC have poor statistics in the region where SRCs are expected 
to be dominant. In addition, the ${ }^{2} \mathrm{D}$ data was taken at a different kinematics compared to the other nuclei which makes the extraction of $a_{2}(A)$ non trivial.

\section{Results from JLab inclusive measurements}

The results from Hall B and Hall C inclusive measurements at JLab are summarized here.

\section{Hall B}

One set of data was taken at Hall B at JLab using the CEBAF Large Acceptance Spectrometer (CLAS) in the kinematic range of $1<x_{b j}<2$ and $1.4<Q^{2}<2.6 \mathrm{GeV}^{2}$ for 2N-SRCs studies. This measurement can be used to compare to SLAC results, described earlier. Data with $x_{b j}>2$ also collected, aiming to study 3N-SRCs [38, 39. For the first time, the cross section ratio of ${ }^{4} \mathrm{He},{ }^{12} \mathrm{C}$ and ${ }^{56} \mathrm{Fe}$ to ${ }^{3} \mathrm{He}\left(R_{3{ }^{H e}}^{A}=\frac{3 \sigma_{A}}{A \sigma_{3} \mathrm{He}}\right)$ were measured under identical kinematic condition. The ratio is shown in Figure 2.2 and it shows scaling (a plateaus) in the region $1<x_{b j}<2$ for $Q^{2}>1.4$, corresponding to scattering from high momentum nucleons. The observation is consistent with the expectation from theory calculation [38] and with the observed scaling in the kinematical region from the SLAC experiment [37]. This scaling behavior seems to be independent of $x_{b j}$ and $Q^{2}$ in the region where 2 N-SRCs dominate $\left(x_{b j}>1.5\right.$ and $Q^{2}>1.4$ ), see Figure 2.2 .

Using the above ratio $R_{3 \mathrm{He}}^{A}$, the probability of finding SRCs in nuclei $A$ relative to ${ }^{3} \mathrm{He}$ can be obtained using the expression [38, 40]:

$$
r\left(A,{ }^{3} \mathrm{He}\right)=R_{{ }^{\mathrm{He}}}^{A} \times \frac{A\left(2 \sigma_{p}+\sigma_{p}\right)}{3\left(Z \sigma_{p}+N \sigma_{n}\right)}
$$

In order to compare to the SLAC results, the per-nucleon probability of finding 

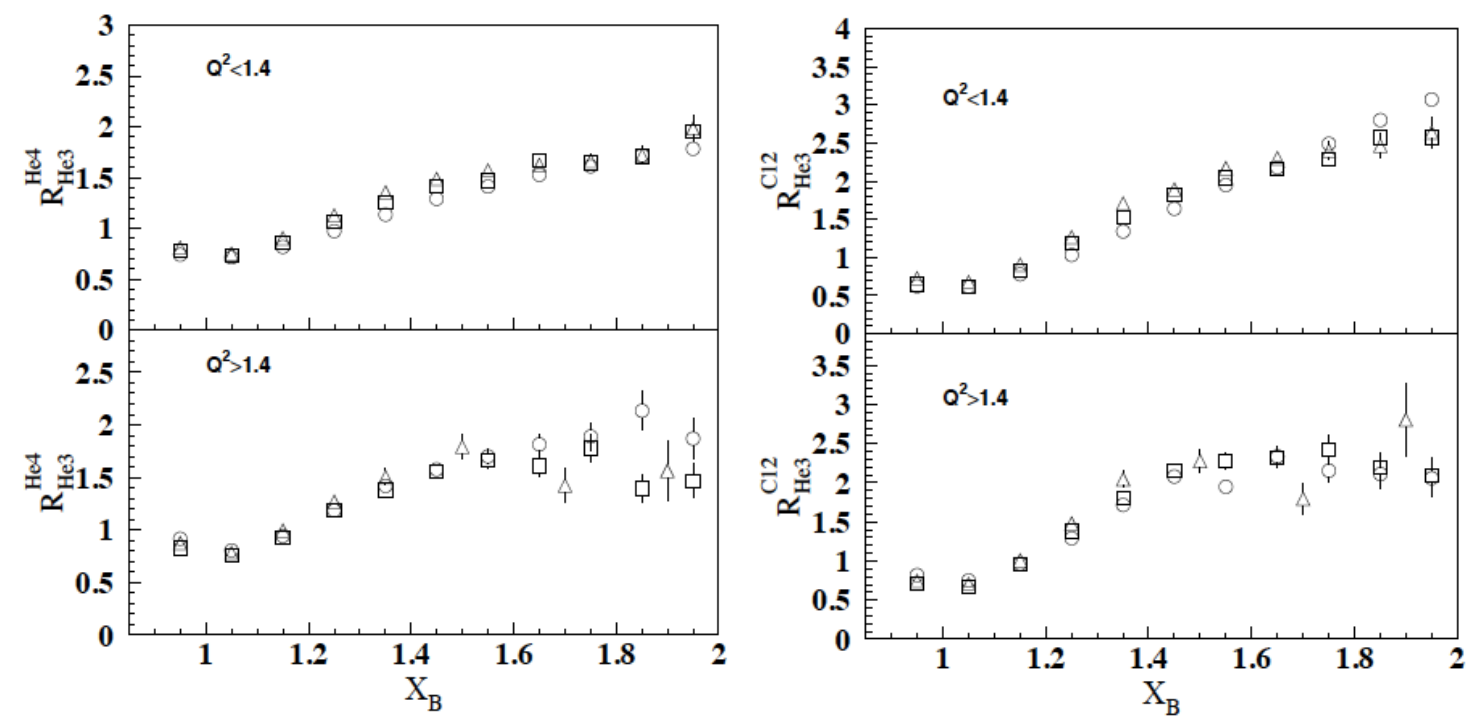

Figure 2.2: The cross section ratio per nucleon of ${ }^{4} \mathrm{He}$ (left plots) and ${ }^{12} \mathrm{C}$ (right plot) to ${ }^{3} \mathrm{He}$. The top plots show the ratio for $Q^{2}<1.4$ without an observation for scaling. The bottom plots show ratio for $Q^{2}>1.4$ with scaling in the region $1<x_{b j}<2[38]$.

SRCs in nuclei $A$ relative to ${ }^{2} \mathrm{D}, a_{2}(A)$, has to be calculated using the relationship:

$$
r\left(A,{ }^{3} \mathrm{He}\right) \sim a_{2}(A) / a_{2}(3) \rightarrow a_{2}(A)=r\left(A,{ }^{3} \mathrm{He}\right) \times a_{2}(3),
$$

where $a_{2}(3)$ is the per-nucleon probability of finding SRCs in ${ }^{3}$ He relative to ${ }^{2} \mathrm{D}$. The value for $a_{2}(3)$ was obtained experimentally $\left(a_{2}(3)=1.7 \pm 0.3[37)\right.$ and using wave function calculations $\left(a_{2}(3)=2 \pm 0.1\right)$ [39]. A weighted average value of $a_{2}(3)=$ $1.97 \pm 0.1$ was taken. The details can be found in Ref. [38]. The results, $a_{2}(A)$, are consistent with the results from SLAC 37].

The ratio $r\left(A,{ }^{3} \mathrm{He}\right)$ as a function of $x_{b j}$ for ${ }^{4} \mathrm{He},{ }^{12} \mathrm{C}$ and ${ }^{56} \mathrm{Fe}$ is presented in Figure 2.3 [39]. The results showed a second plateaus in the region of $2.2<x_{b j}<2.8$. At the time of publication, this was claimed as the first evidence for 3N-SRCs. But the data has its limitations. It was assumed that the $3 \mathrm{~N}-\mathrm{SRCs}$ scaling starts at the same $Q^{2}$ as 2 N-SRCs. These second plateaus included data averaged over the range of $Q^{2}$ and the statistics were dominated by the lowest $Q^{2}$ data. It is difficult to confirm 
that this measurement has reached the region where 3N-SRCs dominate. A detailed discussion of the results can be found in Ref. [41].

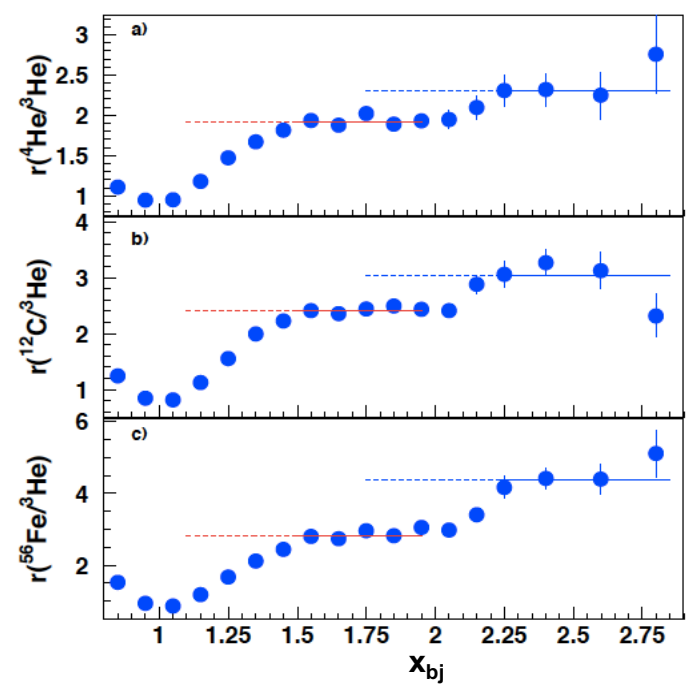

Figure 2.3: The ratio of cross section from different nuclei to ${ }^{3}$ He shows 2 scaling regions. The scaling in the range of $1<\mathrm{x}_{b j}<2$ was claimed as results of $2 \mathrm{~N}$ SRCs, while second scaling $\mathrm{x}_{b j}>2$ was assumed to be the indication of $3 \mathrm{~N}$ SRCs [39].

\section{Hall C}

Along with the data from CLAS, there is also precise data from Hall C. The experiment E02-019 25, 42 performed at JLab's Hall C took data with high precision for $2 \mathrm{~N}$ and $3 \mathrm{~N}-\mathrm{SRCs}$ studies. Information about the experimental setup as well as the results can be found in the Ref. [25, 42]. For $2 \mathrm{~N}$ SRCs studies in the region $1<x_{b j}<2$ , the results are in good agreement to the one from Hall B as well as the SLAC data (see Figure 2.4. A detailed comparison is presented in the Ref. [42].

This experiment also provided a second data set for 3N SRCs in addition to the Hall B data using the ${ }^{4} \mathrm{He} /{ }^{3} \mathrm{He}$ cross section ratio per nucleon (see Figure 2.5). The $Q^{2}$ values from the two data sets are different, $Q^{2} \sim 2.9$ in E02-019 and $Q^{2} \sim 1.6 \mathrm{GeV}^{2}$ for CLAS. The comparison shows a good agreement in the 2N-SRCs region but the E02019 data is significant higher compared to the CLAS data in the region of $x_{b j}>2$. This 


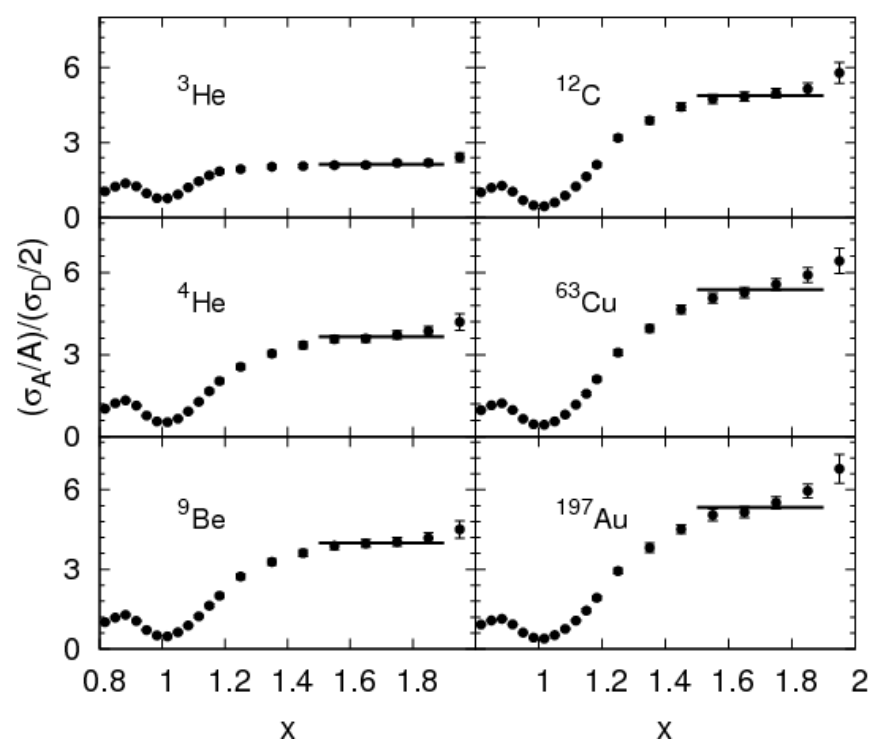

Figure 2.4: The cross section ratio per nucleon for different nuclei to ${ }^{2} \mathrm{D}$ from the E02-019, Hall C at JLab. A clear scaling in the region of 2N SRCs was observed and is in good agreement with the SLAC data 42$]$.

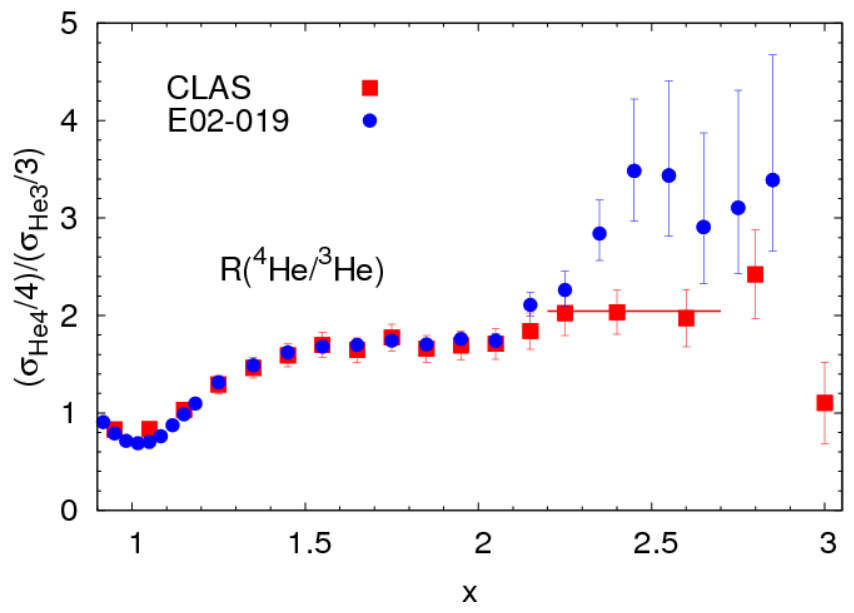

Figure 2.5: The comparison of the cross section ratio $R\left({ }^{4} \mathrm{He} /{ }^{3} \mathrm{He}\right)$ per nucleon between CLAS [39] data and E02-019 data [42].

suggested a $Q^{2}$ dependence of $3 \mathrm{~N}-\mathrm{SRC}$ s and more studies are necessary to determine the kinematic region where the signal can be isolated. 


\section{$2.2 \quad$ Results of Coincident Experiments}

The observed plateaus in the inclusive data is a strong evidence that the high momentum tail in different nuclei has a universal behavior. An exclusive experiment where the scattered electron, scattered proton and recoil nucleon are detected can provide the confirmation that the observed plateaus in the inclusive scattering ratios are due to SRCs. In addition, it can provide more information about the nucleons involved in SRCs. The triple coincident experiment E01-015 was performed in Hall A at JLab to measure the SRC pair directly. The experimental setup is shown in Figure 2.6. ${ }^{12} \mathrm{C}$ was used as the target and data was taken at $x_{b j}=1.2, Q^{2}=2 \mathrm{GeV}$ and missing momentum $P_{m}>300 \mathrm{MeV}$. The scattered electron and proton was detected using the high resolution spectrometers (LHRS and RHRS) of Hall A at JLab. The BigBite and HAND detector packages were installed to detect the recoiling proton and neutron of the pp or pn SRC pair, respectively. There are several important results from this experiment.
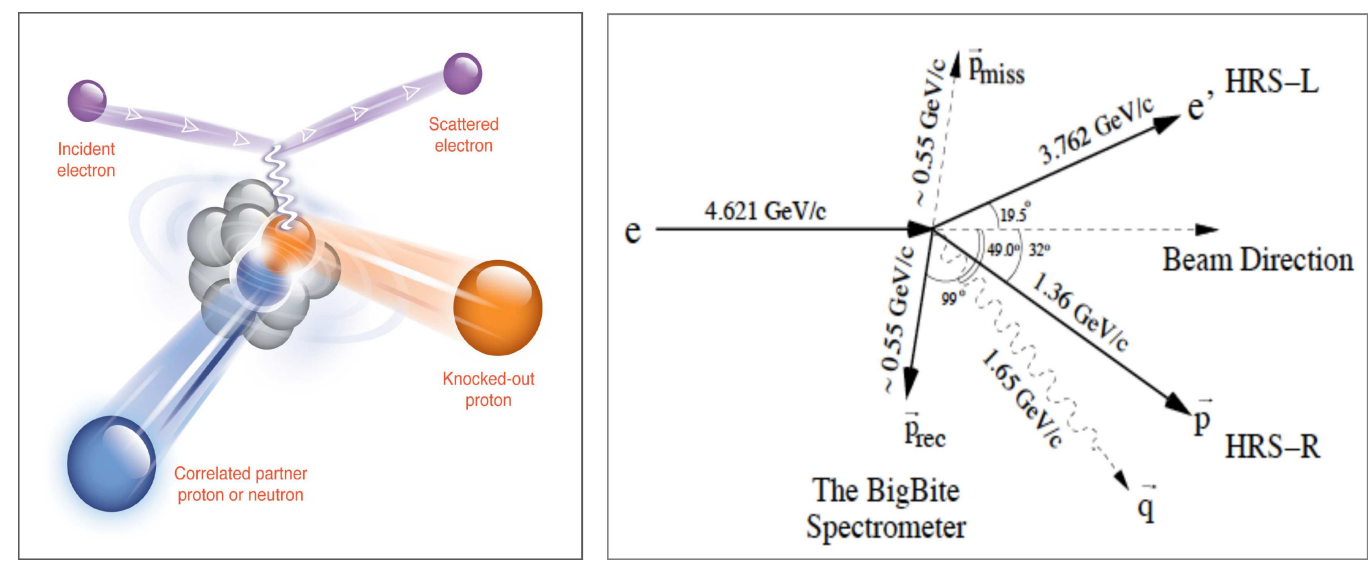

Figure 2.6: The triple coincident experiment E01-015 set up information. The scattering electron, scattered proton and recoiled nucleon were detected in this experiment (left plot). The right plot presents the kinematical settings 43, 44.

The first result is shown in Figure 2.7. It shows the distribution of the cosine of the angle between the missing momentum $\mathbf{p}_{\text {miss }}$ and recoil momentum $\mathbf{p}_{\text {rec }}$ for the $p_{\text {miss }}=$ 
$0.55 \mathrm{GeV}$ kinematics. The data showed that most of the time a scattered proton with high momentum was detected, a recoil proton or neutron was also detected with similar momentum in almost the opposite direction. This indicates that the high momentum nucleons come from correlated pairs with small total momentum and high relative momentum (a SRCs pair). Because of this small total momentum, the recoiling nucleons come out with a large angle, nearly $\mathrm{n}$ the opposite direction with respect to the scattered proton direction.

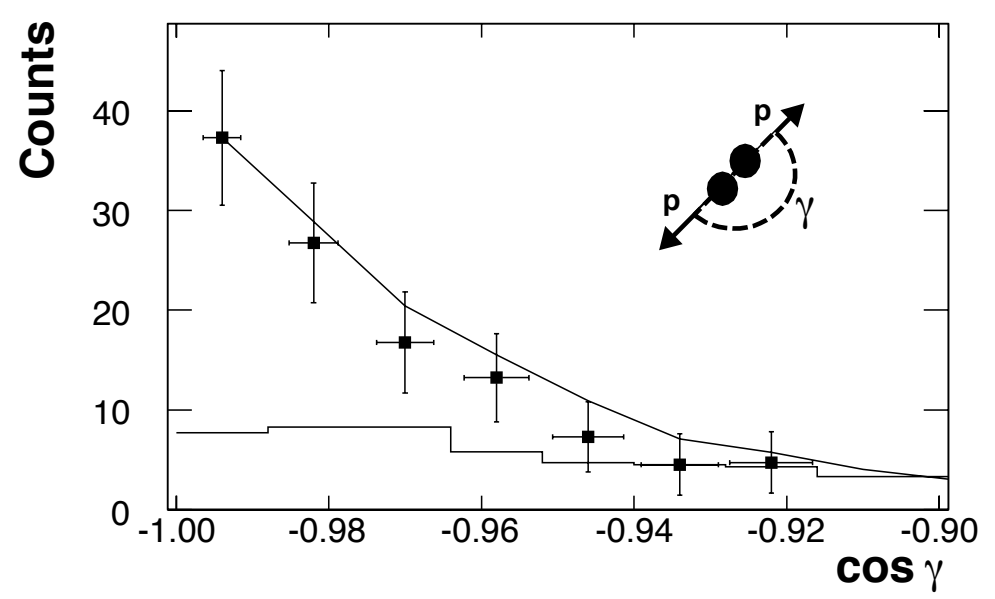

Figure 2.7: The $\cos \left(\mathbf{p}_{\text {miss }} \mathbf{p}_{\text {rec }}\right)$ distribution shown as points [43]. The histogram presents the events distribution from random event selection and the curve is the expectation based on simulation.

The second important result from the triple E01-015 is the evidence of the isospin dependence of SRCs.

\section{Isopspin dependence of SRC}

In the simple model, SRCs are assumed to be isospin-independent, with equal contributions from different types of correlated pairs (pp, nn, np). E01-015 provided the ratio of $\left(e^{\prime} e^{\prime} p p\right) /\left(e, e^{\prime} p\right)$ and $\left(e, e^{\prime} p n\right) /\left(e, e^{\prime} p\right)$ since for every event (e,e'p), the recoil proton or neutron is also detected. The results show that most of the (e,e'p) events $(96 \% \pm 22 \%)$ with a $p_{\text {miss }}>300 \mathrm{MeV}$ have a recoil neutron [44. The SRC measure- 
ments show that SRC contribute to $20 \%$ of the ground state wave function while $90 \%$ of them come from np pairs (see Figure 2.8). A consistent result was obtained from an independent experiment performed at BNL using a proton beam to measure the $(\mathrm{p}, 2 \mathrm{pn}) /(\mathrm{p}, 2 \mathrm{p})$ ratio. Results of that experiment showed that $92 \% \pm 18 \%$ of the events $(\mathrm{p}, 2 \mathrm{p})$ with a $p_{\text {miss }}>275 \mathrm{MeV}$ were detected with a correlated recoiled neutron, see Figure 2.8 (left) 45 .
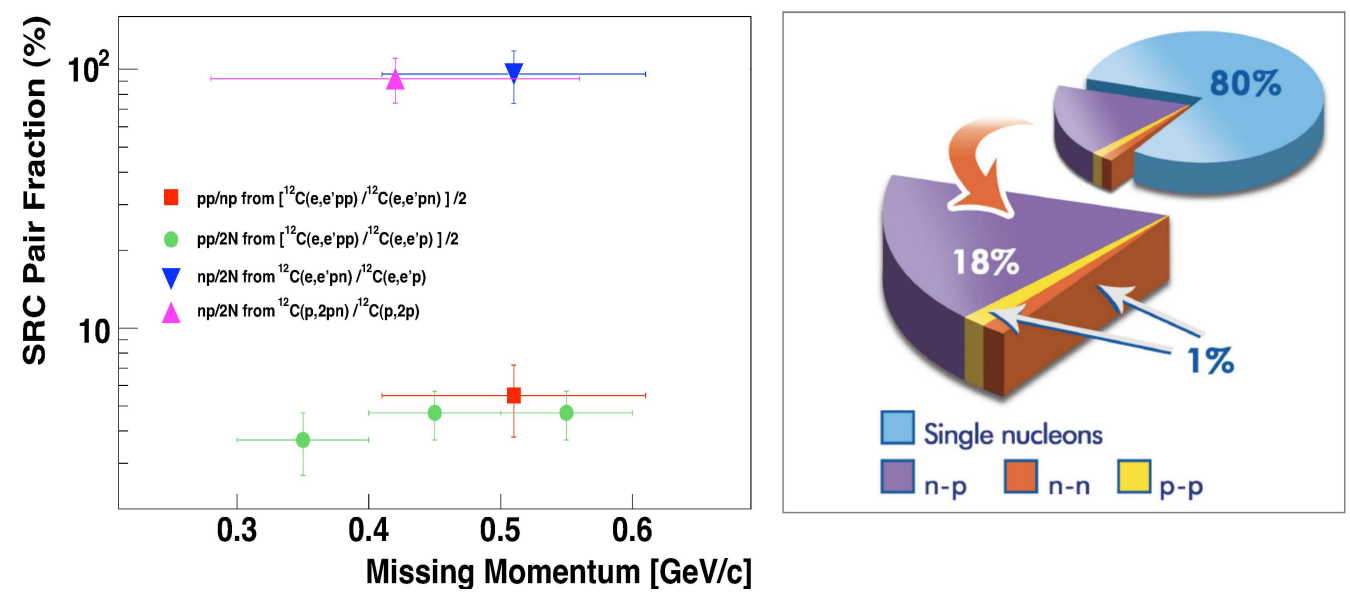

Figure 2.8: Left plot: the fraction of correlated pair combination in ${ }^{12} \mathrm{C}$ as obtained from the (e,e'pp) and (e,e'pn) reaction [44], as well as from the (p,2pn) data [45]. Right plot: the average fraction of nucleons in the various initial configuration of ${ }^{12} \mathrm{C} 44$.

This is a strong evidence for the isospin dependence of SRCs. This dependence has been explained by several theoretical calculations. Figure 2.9 shows the momentum distribution along with contribution of np pairs (solid line) and the pp pairs (dash line) for different nuclei from calculations [46]. These calculations clearly show that in the region where SRCs are dominant, the contribution from np pairs is much larger when compared to the one from pp pairs.

The isospin-dependent results motivated many other experiments to seek a better understanding. Some of them will be presented in the Section 2.4 . 


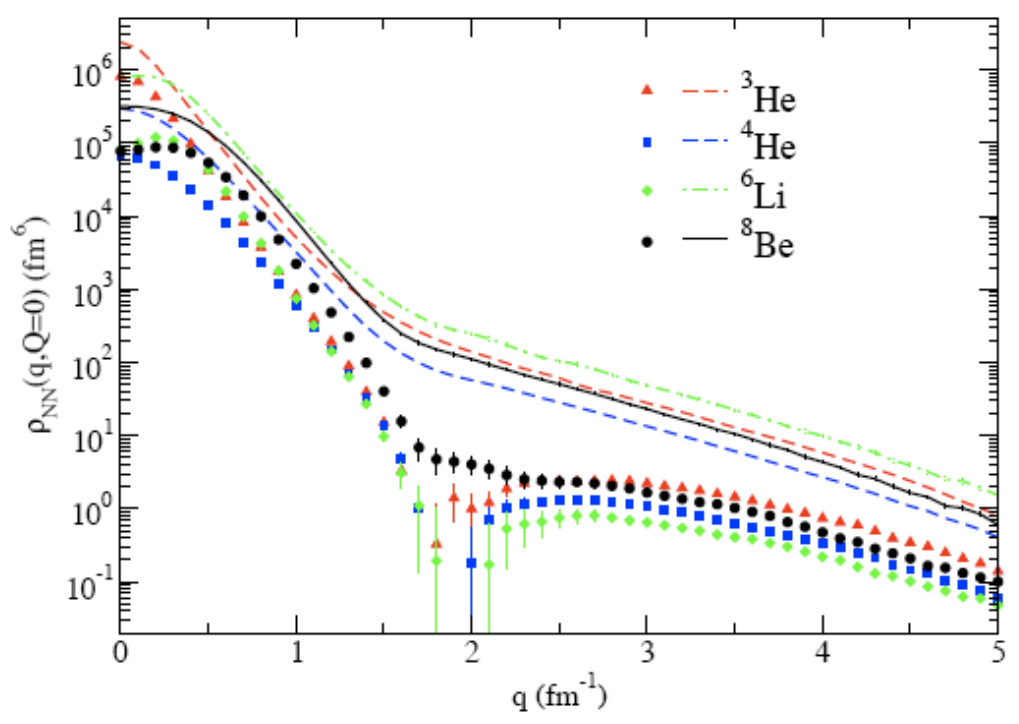

Figure 2.9: The nucleon momentum distribution calculated for different nuclei, indicated in the plots [46]. np contributions are shown by the solid line and pp contributions are shown by dashed line. The np pairs have a much larger contribution compared to pp pairs in the region where SRC expected to dominate.

\section{Momentum sharing in imbalanced Fermi system}

After isospin-dependence was revealed in triple coincident experiment using ${ }^{12} \mathrm{C}$, another coincident measurment was done in Hall B at JLab using the CLAS detector on different nuclei ${ }^{12} \mathrm{C},{ }^{27} \mathrm{Al},{ }^{56} \mathrm{Fe}$ and ${ }^{208} \mathrm{~Pb}$. This experiment detected (e,e'pN) events with kinematics chosen to ensure that the knock-out proton belonged to the SRC pairs $\left(Q^{2}>1.5 \mathrm{GeV}^{2}\right.$ and $\left.x_{b j}>1.2\right)$ and missing momentum $\left.300<p_{m}<600 \mathrm{MeV}\right)$. This was the first attempt to investigate the isospin-dependence of nuclei with $A>12$. The dominance of np SRC pairs over pp SRC pairs appear to be a universal property for every measured nuclei (see Figure 2.10] [47]. With np dominance, the number of protons and neutrons having high momentum are the same. So in neutron-rich nuclei the fraction of neutrons with high momentum is smaller than the fraction of protons which gives the protons a higher average momentum compared to neutrons. This result is in opposition to what is expected from the Pauli principle. Without the SRC interaction the Pauli principle will push the majority nucleons to higher aver- 


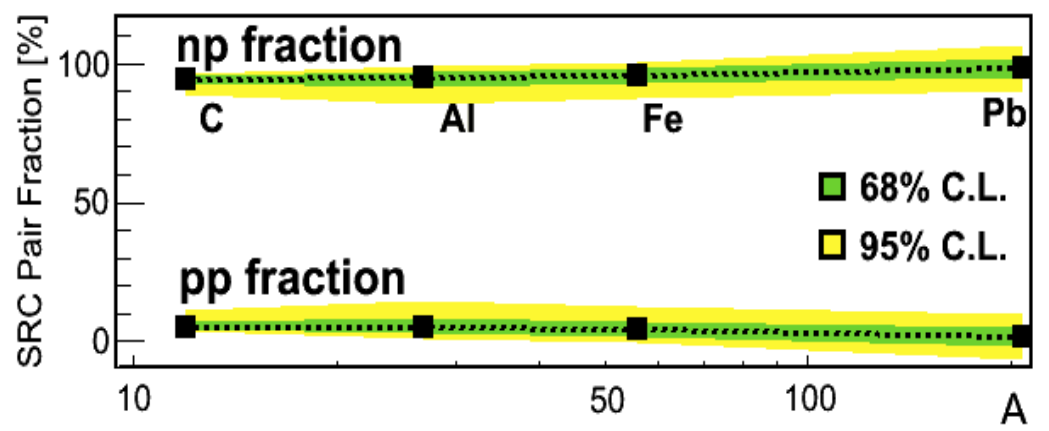

Figure 2.10: The evidence of the isospin-dependence for heavy nuclei $A>12$. The plots show the fraction of np and pp SRCs pair. The yellow and green bands show the confident levels. The np SRCs pair fraction is dominant over pp SRCs pair in all measured nuclei, Ref 47].

age momentum. This would imply that in neutron-rich nuclei the majority nucleon, neutron, would have higher average momentum.

For light nuclei $(A<12)$ the average kinetic energy of a proton and neutron can be calculated by integrating the single-nucleon momentum distribution over the range of momenta.

$$
\left\langle T_{p(n)}\right\rangle=\int n_{p(n)}(k) \cdot \frac{k^{2}}{2 m} \cdot d^{3} \vec{k},
$$

where $\left\langle T_{p(n)}\right\rangle$ is the average kinetic energy of the proton (neutron). $n_{p(n)}(k)$ is the proton (neutron) momentum distribution obtained by many-body Variational Monte Carlo calculations (VMC) which use AV18 potential and Urbana-X potential for $2 \mathrm{~N}$ and $3 \mathrm{~N}$ interactions, respectively. The $n_{p(n)}(k)$ distribution needs to be normalized to 1 .

$$
\int n_{p(n)}(k) \cdot d^{3} \vec{k}=1
$$

The results of this calculation, presented in Ref. [9], showed that the average kinetic energy of the minority nucleons is larger than the one from the majority nucleons. This is consistent with the expectation of np pair dominance in the high momentum tail. But this calculation is only available for light nuclei. In order to explain the np-dominance observed in heavy nuclei, an effective momentum distribution (con- 
tact formalism) is used in calculations. The basic idea of this effective momentum distribution can be understood using a toy model, described in Figure 2.11.

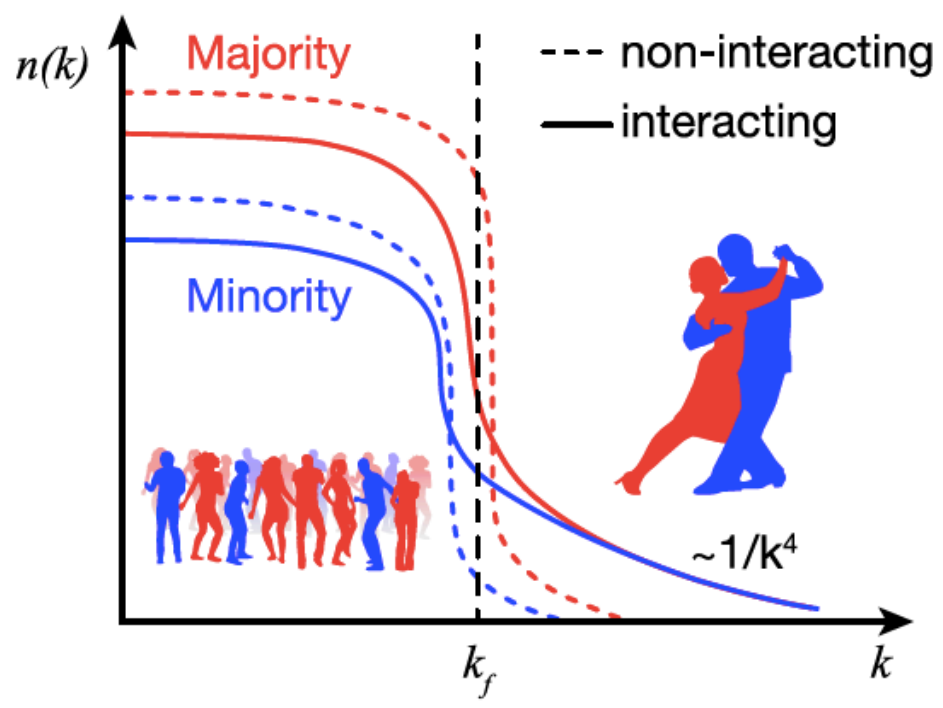

Figure 2.11: The momentum distribution in an imbalanced Fermi system. Without SRC interaction (dashed line), the majority nucleon has a higher average momentum. Including the SRCs interaction (solid line) high momentum tail is created. The same number of majority and minority nucleons have high momentum which leaves a larger fraction of majority nucleon at the low momentum states 47 .

The solid lines and dashed lines represent nucleon momentum distribution with and without SRC interactions, respectively. The interacting nucleon momentum distribution has the same number of minority and majority nucleon which leads to a larger fraction of majority nucleons in low momentum states. With this assumption, the momentum distribution $n_{p(n)}(k)$ of protons and neutrons in heavy nuclei can be expressed as follows:

$$
n_{p(n)}(k)= \begin{cases}\eta \cdot n_{p(n)}^{\text {mean-field }}(k) & k<k_{0} \\ \frac{A}{2 Z(N)} \cdot a_{2}(A) \cdot n_{d}(k) & k>k_{0}\end{cases}
$$

where $n_{p(n)}^{\text {mean-field }}$ is the mean-field momentum distribution of the proton (neutron) in the nucleus $A$ with $Z$ protons and $N$ neutrons. The scaling factor $a_{2}(A)$ is the ratio 
of nuclei $A$ cross section to ${ }^{2} \mathrm{D}$ in the $2 \mathrm{~N}$ SRC region extracted from data, and the $n_{d}(k)$ is ${ }^{2} \mathrm{D}$ the momentum distribution.

Integrating the above momentum distributions over the momentum range, the average kinetic energy of a proton and neutron in different nuclei can be obtained. The detailed calculation can be found in Ref. [9]. The summary of the results are shown in Figure 2.12.

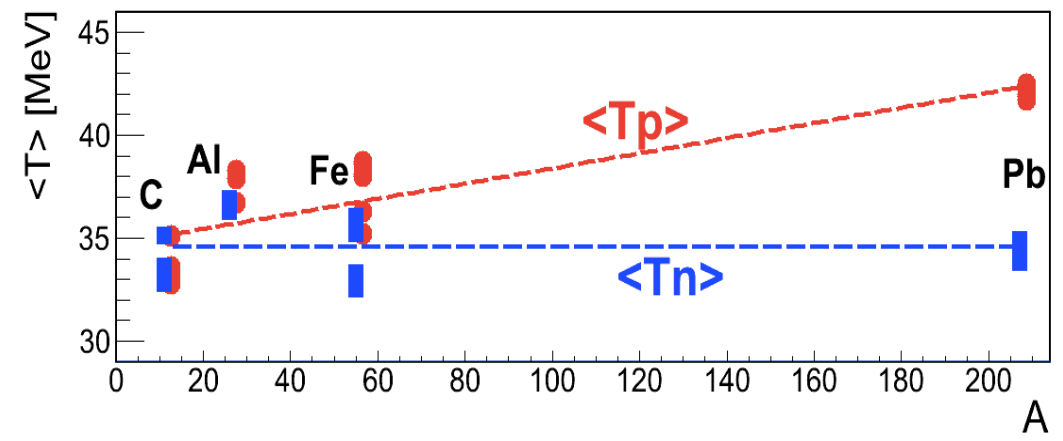

Figure 2.12: The average kinetic energy of proton and neutron calculations in different nuclei using the momentum distribution in the Equation 2.5.

The results showed that by including the np-dominance in the calculation the average kinetic energy of protons is larger than that of neutrons for heavy nuclei. This is consistent with the np-dominance observed in different heavy nuclei.

\subsection{SRCs and The EMC effect}

\section{The EMC effect}

Whether the quark structure in a bound nucleon and a free nucleon is the same is another outstanding question in nuclear physics. The general expectation is that they are the same. In the 1980s, Deep Inelastic scattering (DIS) measurements were performed at CERN to extract the DIS structure function $F_{2}$ for both heavy nuclei $(\mathrm{Fe}, \mathrm{Cu})$ and ${ }^{2} \mathrm{D}$. The experimental data shows that the deep inelastic structure 
function $F_{2}$ for heavy nuclei was not the same as for ${ }^{2} \mathrm{D} 48$. In other words, the ratio of $F_{2}^{A} / F_{2}^{D}$ is not equal to 1 as expected in the region $0.3<x_{b j}<0.7$, where the Fermi motion can be ignored. It is noteworthy that the binding energy is extremely small (few MeV) compared to the transferred energy (several GeV) in this experiment and therefore no noticeable effect was expected. In Figure 2.13 , the ratio $F_{2}^{F e} / F_{2}^{D}$ is shown for data along with theoretical prediction [48]. The observed difference to the expectation is referred as the EMC (European Muon Collaboration) effect. This suggests that a bound nucleon inside nucleus behaves differently from a free nucleon.

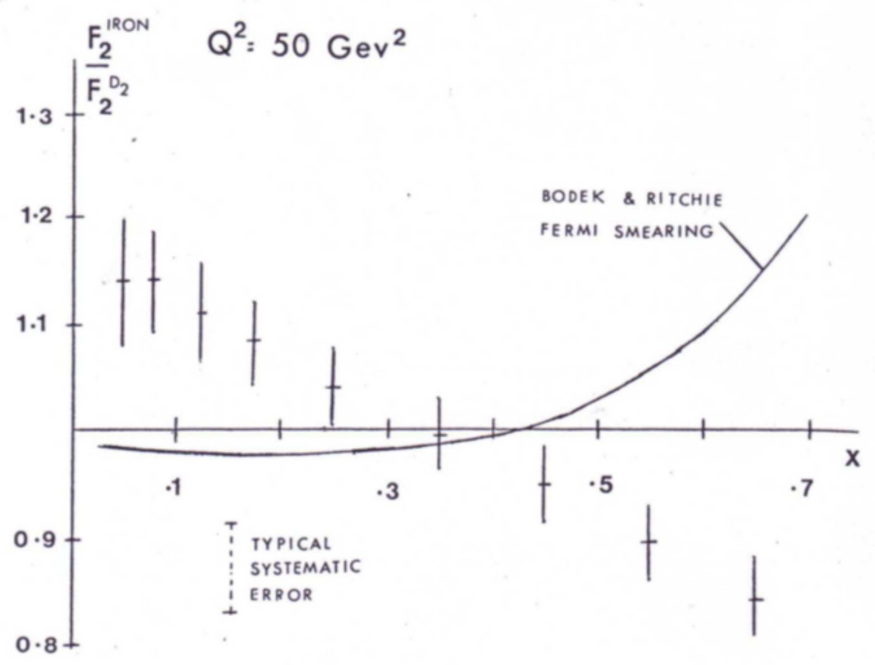

Figure 2.13: The DIS structure function $F_{2}$ ratio, $F_{2}^{F e} / F_{2}^{D}$, from data at CERN, and thetheoretical expectation 48.

After the first observation, there were more experiments conducted to study this effect in different nuclei [49,50]. The results can be seen in Figure 2.14 where the DIS cross sections ratio per nucleon $(R)$ for different nuclei to the ${ }^{2} \mathrm{D}$ is plotted as a function of $x_{b j}$ for the SLAC data (left plots) [49] and JLab data (right plots) [50]. The EMC effect appears to be universal in different nuclei.

The strength of the EMC effect is taken as the slope of the ratio $R\left(d R_{E M C} / d x\right)$ 

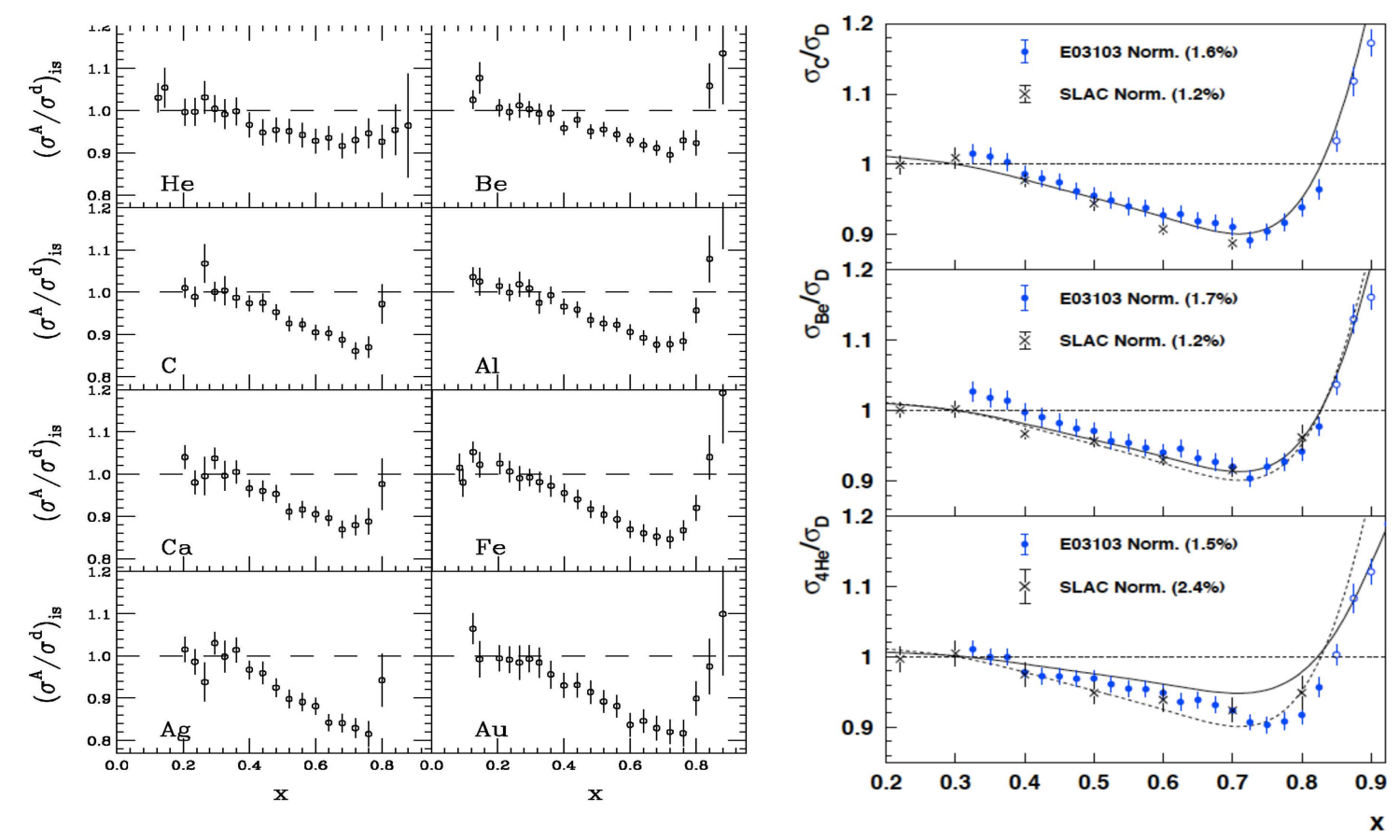

Figure 2.14: The DIS cross section ratio per nucleon for different nuclei to the ${ }^{2} \mathrm{D}$. The left plots present SLAC data [49] while the right plots present JLab data [50]. The results show the EMC effect is universal in different nuclei..

in the region $0.3<x_{b j}<0.7[50]$. There are many phenomelogical explanations for the EMC effect but no general accepted solution so far. The next section will discuss about a possible explanation of the EMC effect related to SRCs.

\section{Possible connection between SRCs and the EMC effect}

As discussed above, the EMC effect was observed in DIS measurement in the region $0.3<x_{b j}<0.7$ and $Q^{2}>2$ which it is sensitive to the nuclear quark distributions. The nuclear scaling was observed in the quasi-elastic region $x_{b j}>1.5$ and $Q^{2}>1.5$ which is sensitive to SRCs. While the EMC effect is thought to be related to the medium modification, the SRCs are related to the high momentum tail in the wave function. They seem to be independent effects. But both of them are related to the local density (see Refs. [51 53]). A quantitative comparison of the strength of the EMC effect and SRC scaling factors for different nuclei was performed in Refs. [51,53. 
and the results are shown in Figure 2.15.
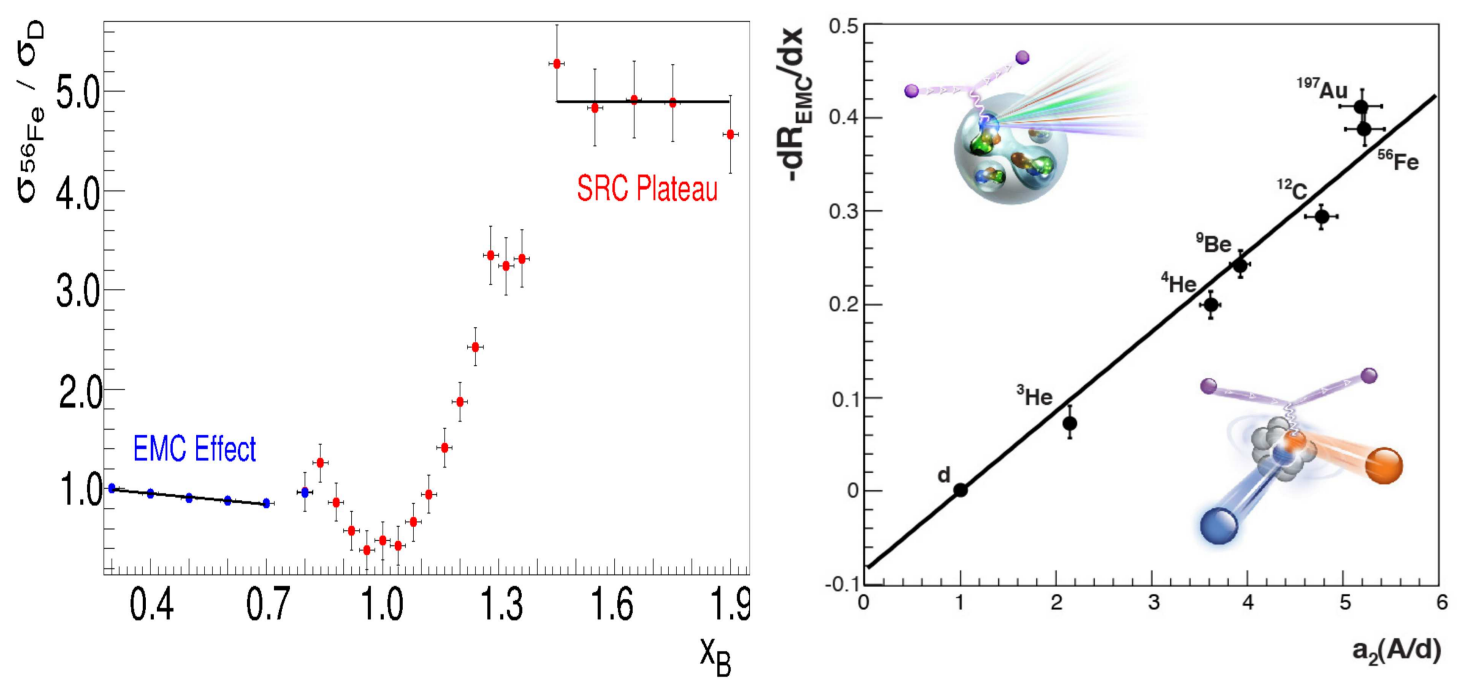

Figure 2.15: A quantitative comparison of the EMC strength and SRC scaling factors for different nuclei. The left plot shows the cross section ratio per nucleon for Fe to ${ }^{2} \mathrm{D}$ as function of $x_{b j}$. The EMC effect and SRC scaling both is visible. The right plot shows the correlation between the EMC strength and the SRC scaling over a range of nuclei.

A strong linear correlation between the EMC strength, $d R_{E M C} / d x$, and the SRC scaling factors, $a_{2}(A)$, suggested that they are related. This correlation is the first experimental clue about a common cause of the EMC effects and SRCs

The study of SRCs is still on going to improve out knowledge of the nuclear structure at short distances. The next section will discuss the motivation for E08-014.

\subsection{Motivations of Experiment E08-014}

The E08-014 was proposed and approved in 2008. This experiment collected data in the kinematic region $1.3<Q^{2}<2.2$ and for both $x_{b j}>1$ and $x_{b j}>2$. A more detailed information about the experimental setup as well as the kinematical setting will be presented in Chapter 3. This experiment was ran in Hall A at JLab in 2011 and it focus on two main goals which are 1) the study of 3N-SRCs in the region 
$x_{b j}>2$ using the cross section ratio ${ }^{4} \mathrm{He} /{ }^{3} \mathrm{He}$ and 2) the isospin-dependence for $2 \mathrm{~N}$ SRCs in region $1<x_{b j}<2$ using the ratio of cross section ${ }^{48} \mathrm{Ca} /{ }^{40} \mathrm{Ca}$. Each goal will be discussed in the following sections

\section{N SRCs study using the ratio ${ }^{4} \mathrm{He} /{ }^{3} \mathrm{He}$}

At the time the experiment was proposed, there were two sets of data (see Refs. [39, 42]) in the region $x_{b j}>2$ where $3 \mathrm{~N}-\mathrm{SRC}$ s were expected to be dominant, but they did not agree with each other (see Section 2.1). The original motivation for this experiment was to collect data in the same kinematic region to provide a third data set for $3 \mathrm{~N}-\mathrm{SRC}$ s studies with higher statistics and a $Q^{2}$ dependence check. This study was carried out by a previous analysis [54] and the result was published in Ref. [55 (see Figure 2.16)

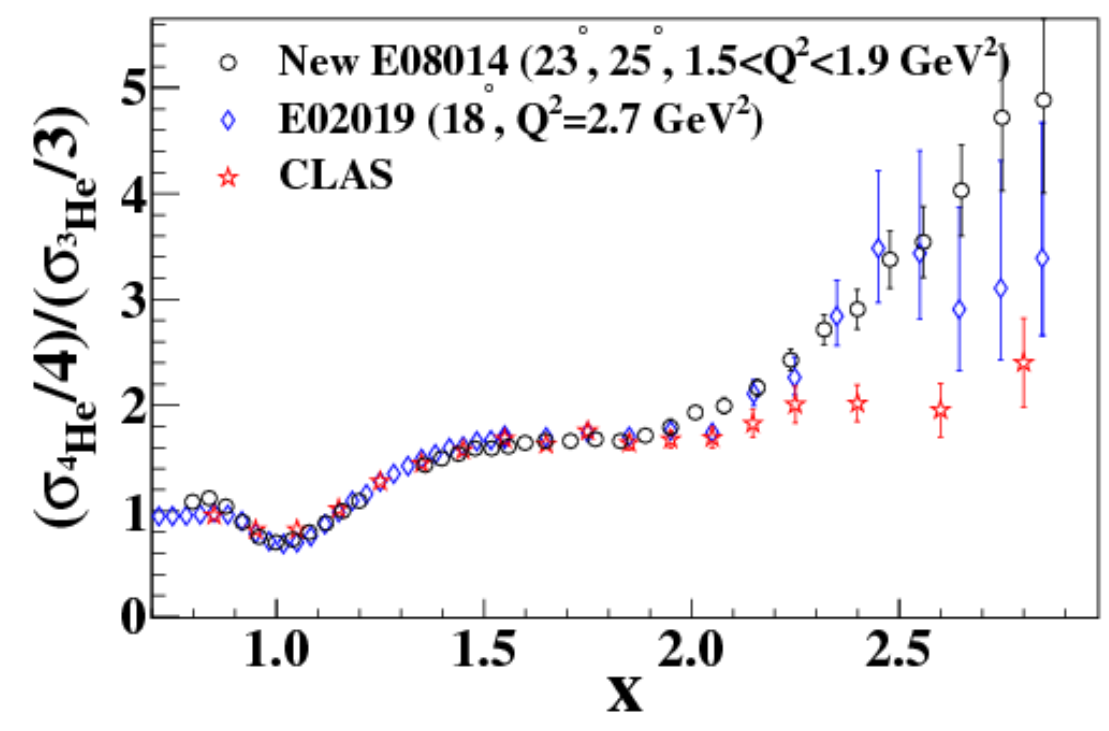

Figure 2.16: A comparison of 3 different data sets for 3N-SRCs studies from JLab's hall A, B and C. They are in good agreement in the 2N-SRCs region $1<x_{b j}<2$. For the 3N-SRCs region $x_{b j}>2$, the data from E08-014 (hall A) and E02-019 (hall C) agree with each other but differ from the CLAS (hall B) data [55]

The results gave a very good agreement with both the CLAS (Hall B) and E02- 
019 (Hall C) data in the 2N-SRCs region $1<x_{b j}<2$. But in the region where 3N-SRCs are expected to be observed, the E08-014 data is significantly higher than the CLAS data and consistent with the E02-019 data. This supports the comments on the CLAS data in Ref. [56] that the second scaling in the CLAS data for $x_{b j}>2$ is due to the poor energy resolution and a large bin migration.

Evidence for 3N-SRCs is still an open question and one possibility is that much higher $Q^{2}$ is required to be able to observe the second scaling, as discussed in Ref. 35. These results also suggest that it is much more difficult to isolate $3 \mathrm{~N}$ SRCs, though there are preliminary indication of 3N SRCs, also in Ref [35].

\section{The 2N SRCs isospin-dependence in $A\left(e, e^{\prime}\right)$}

The other goal of the experiment, also the focus of this thesis, is the isospin-dependence of $2 \mathrm{~N}$ SRCs using the per-nucleon cross section ratio ${ }^{48} \mathrm{Ca} /{ }^{40} \mathrm{Ca}$. With and without the isospin-dependence assumption this ratio would return different results. With the isospin-independent assumption, the ratio of the number of neutron to number of proton in SRCs is equal to the $\mathrm{N} / \mathrm{Z}$ ratio of nucleus. The isospin dependence of SRCs can be studied using nucleus with different $\mathrm{N} / \mathrm{Z}$ ratios. In the kinematics of E08014 (see Chapter 3), the cross section of electron-proton scattering $\left(\sigma_{p}\right)$ is about 3 times the electron-neutron scattering $\left(\sigma_{n}\right)$. In the isospin-independent assumption, the cross section ratio per-nucleon from ${ }^{48} \mathrm{Ca}$ to ${ }^{40} \mathrm{Ca}$ can be expressed as [41]:

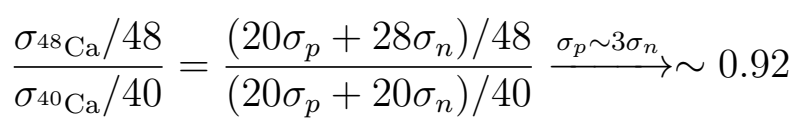

In the case of the isospin-dependence, there is not yet a precise estimation of this effect on the cross section ratio per-nucleon for ${ }^{48} \mathrm{Ca} /{ }^{40} \mathrm{Ca}$ due to the complexity of these nuclear systems. Only a hand waving estimation was used for this ratio based on the total possible combination of np pairs, $(Z * N)$, presented in the proposal [41]: 


$$
\frac{\sigma^{48} \mathrm{Ca} / 48}{\sigma^{40} \mathrm{Ca} / 40}=\frac{(20 \cdot 28) / 48}{(20 \cdot 20) / 40}=1.17
$$

Later a theoretical calculation was provided using a realistic potential and taking into account the isospin-dependence effect it aimed to quantify short-range correlations in nuclei [57]. The results from this calculation can predict the fraction np pairs prone to SRC from the total possible number of np pair combinations as function of nuclear mass $A$, including ${ }^{40} \mathrm{Ca}$ and ${ }^{48} \mathrm{Ca}$, see Figure 2.17. Using this prediction with

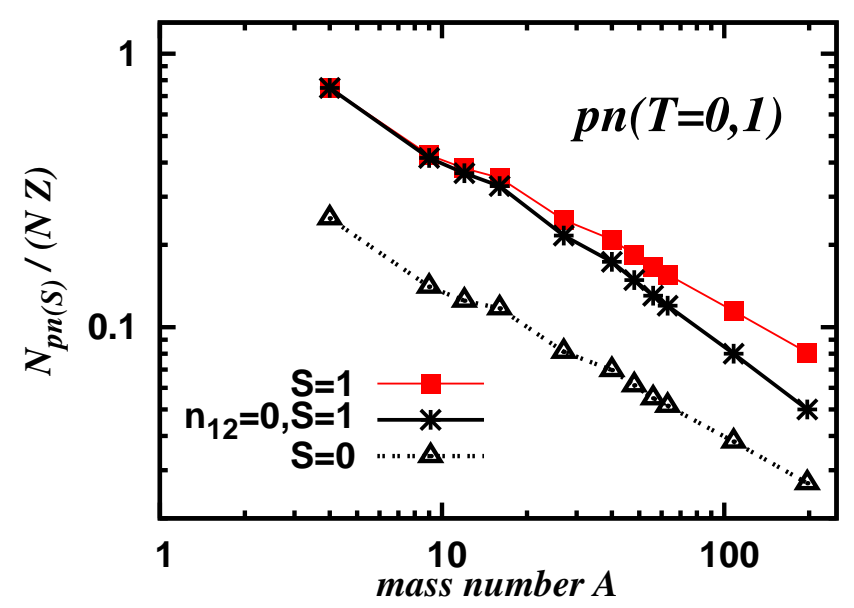

Figure 2.17: The predicted fraction of $\mathrm{np}$ pairs are prone to SRCs from the total number combination of np pairs $(N * Z)$ for ${ }^{4} \mathrm{He},{ }^{9} \mathrm{Be},{ }^{12} \mathrm{C},{ }^{27} \mathrm{Al},{ }^{40} \mathrm{Ca},{ }^{48} \mathrm{Ca},{ }^{56} \mathrm{Fe}$, ${ }^{63} \mathrm{Cu},{ }^{108} \mathrm{Ag}$ and ${ }^{197} \mathrm{Au}[57]$.

the isospin-dependent assumption the ratio of cross section per nucleon ${ }^{48} \mathrm{Ca} /{ }^{40} \mathrm{Ca}$ is around 1 .

$$
\frac{\sigma_{48} \mathrm{Ca} / 48}{\sigma^{40} \mathrm{Ca} / 40} \sim 1
$$

The detailed analysis of this isospin-dependence study will be presented in the following chapters. The results and its implication can be found in Chapter 6 .

A new SRC measurement, E12-11-112 [58] is running using the mirror targets ${ }^{3} \mathrm{He}$ and ${ }^{3} \mathrm{H}$ to measure the $2 \mathrm{~N}$ SRCs region. It will provide a very clear probe of isospindependence. In these $A=3$ mirrored nuclei, the effect of isospin-dependence on the 
cross section can be easily estimated. In the isospin-independent case the cross section ratio $\sigma_{3} \mathrm{He} / \sigma_{3} \mathrm{H}$ can be expressed as $\left(2 \sigma_{p}+\sigma_{n}\right) /\left(\sigma_{p}+2 \sigma_{n}\right) \sim 1.4$ with the condition $\sigma_{p} \sim 3 \sigma_{n}$. In the isospin-dependent case the cross section ratio is $1, \sigma^{3} \mathrm{He} / \sigma_{3} \mathrm{H}=1$ because both of these nuclei have two np pairs. There is a $40 \%$ difference in the cross section ratio using these different assumptions. This is very large and should be easy to detect. The results will provide more information about isospin dependence in light nuclei. 


\section{Chapter 3}

\section{The Experiment Setup}

\subsection{Overview}

This chapter will give an introduction to the setup of E08014 41, 54. Only a short introduction to the accelerator, the beam line components and detector package in the Hall A will be presented here.

\section{$3.2 \quad \mathrm{E} 08014$}

E08014 (also known as $x>2$ ) was performed in Hall A of the Thomas Jefferson National Accelerator Facility (JLab) during the Spring 2011. A single beam energy of $3.356 \mathrm{GeV}$ was used during the experiment to do inclusive measurements at 9 kinematic settings on 6 different targets: ${ }^{2} \mathrm{D},{ }^{3} \mathrm{He},{ }^{4} \mathrm{He},{ }^{12} \mathrm{C},{ }^{40} \mathrm{Ca}$ and ${ }^{48} \mathrm{Ca}$. Each kinematic setting is defined by the central scattering angle angle $\theta_{0}$ and central spectrometer momentum $P_{0}$ of the spectrometer. Table 3.1 presents $\theta_{0}, P_{0}$ and the central kinematic variables $\mathrm{Q}_{0}^{2}, \mathrm{x}_{b j}^{0}$ for each kinematic setting. The kinematic range of $\mathrm{Q}^{2}$ and $\mathrm{x}_{b j}$ is shown on Figure 3.1 . 
Table 3.1: The central kinematic variables for each kinematic setting, including central momentum $\left(P_{0}\right)$, central angle $\left(\theta_{0}\right), Q_{0}^{2}$ and $x_{b j}^{0}$.

\begin{tabular}{||c|c|c|c|c|c|c|c|c|c|c||}
\hline \hline Kin & K3.1 & K3.2 & K4.0 & K4.1 & K4.2 & K5.0 & K5.05 & K5.1 & K5.2 & K6.5 \\
\hline $\mathrm{P}_{0}(\mathrm{GeV})$ & 2.905 & 3.055 & 2.6 & 2.855 & 3.035 & 2.505 & 2.650 & 2.795 & 2.995 & 2.845 \\
\hline$\theta_{0}\left(^{\circ}\right)$ & 21 & 21 & 23 & 23 & 23 & 25 & 25 & 25 & 25 & 28 \\
\hline$Q_{0}^{2}\left(\mathrm{GeV}^{2}\right)$ & 1.29 & 1.36 & 1.38 & 1.5 & 1.61 & 1.57 & 1.66 & 1.75 & 1.88 & 2.23 \\
\hline $\mathrm{x}_{b j}^{0}$ & 1.53 & 2.41 & 0.97 & 1.62 & 2.69 & 0.98 & 1.26 & 1.67 & 2.78 & 2.33 \\
\hline
\end{tabular}

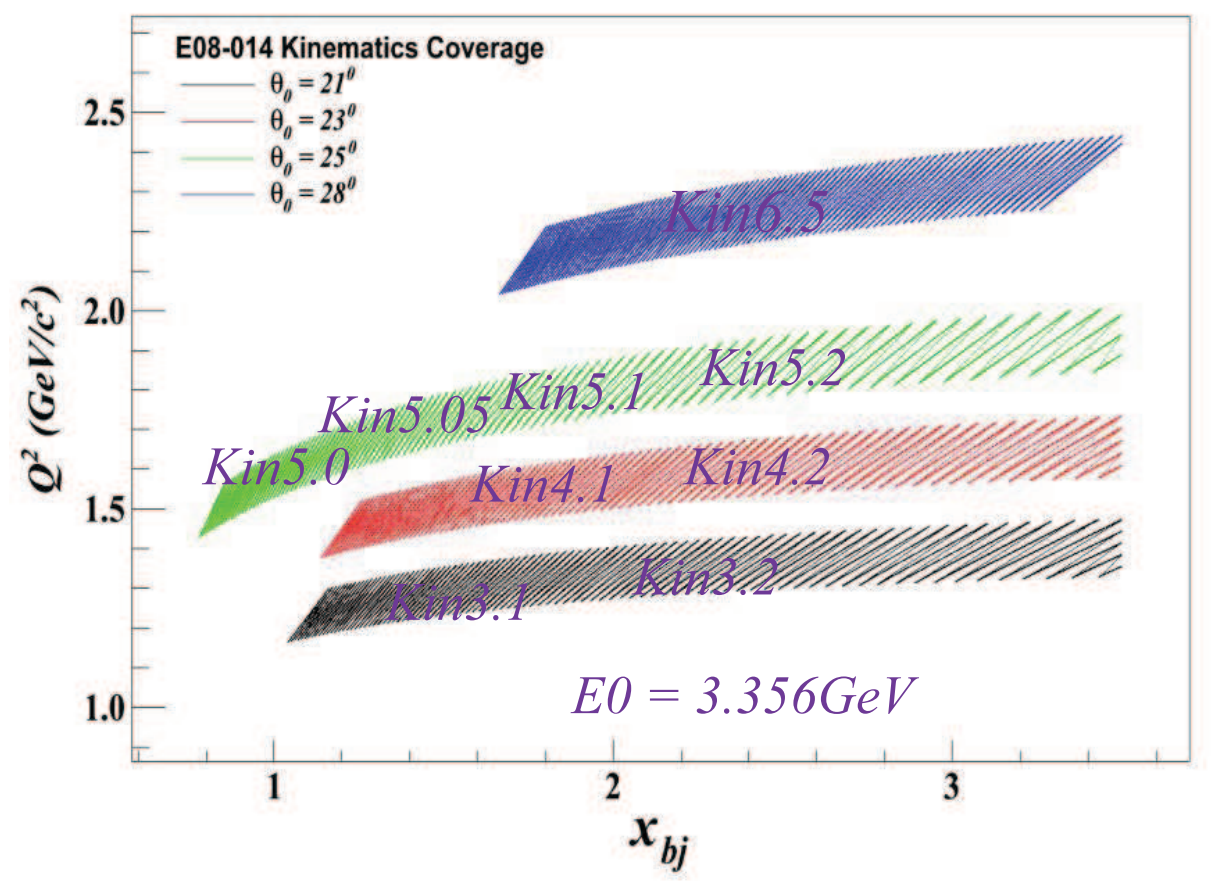

Figure 3.1: Kinematic coverage for every settings as a function of $\mathrm{Q}^{2}$ and $\mathrm{x}_{b j}$. The beam energy is fixed for all kinematic settings. Each color band indicates a fixed angle and the associated range of momentum. 


\subsection{CEBAF}

The Continuous Electron Beam Accelerator Facility (CEBAF) at Jefferson Lab was designed to deliver high quality electron beams simultaneously to three different experimental Halls A, B, C [59]. The recent $12 \mathrm{GeV}$ upgrade increases the maximum beam energy from $6 \mathrm{GeV}$ to $12 \mathrm{GeV}$ and added another experimental, Hall D. The layout of the experimental halls is shown in Figure 3.2 .

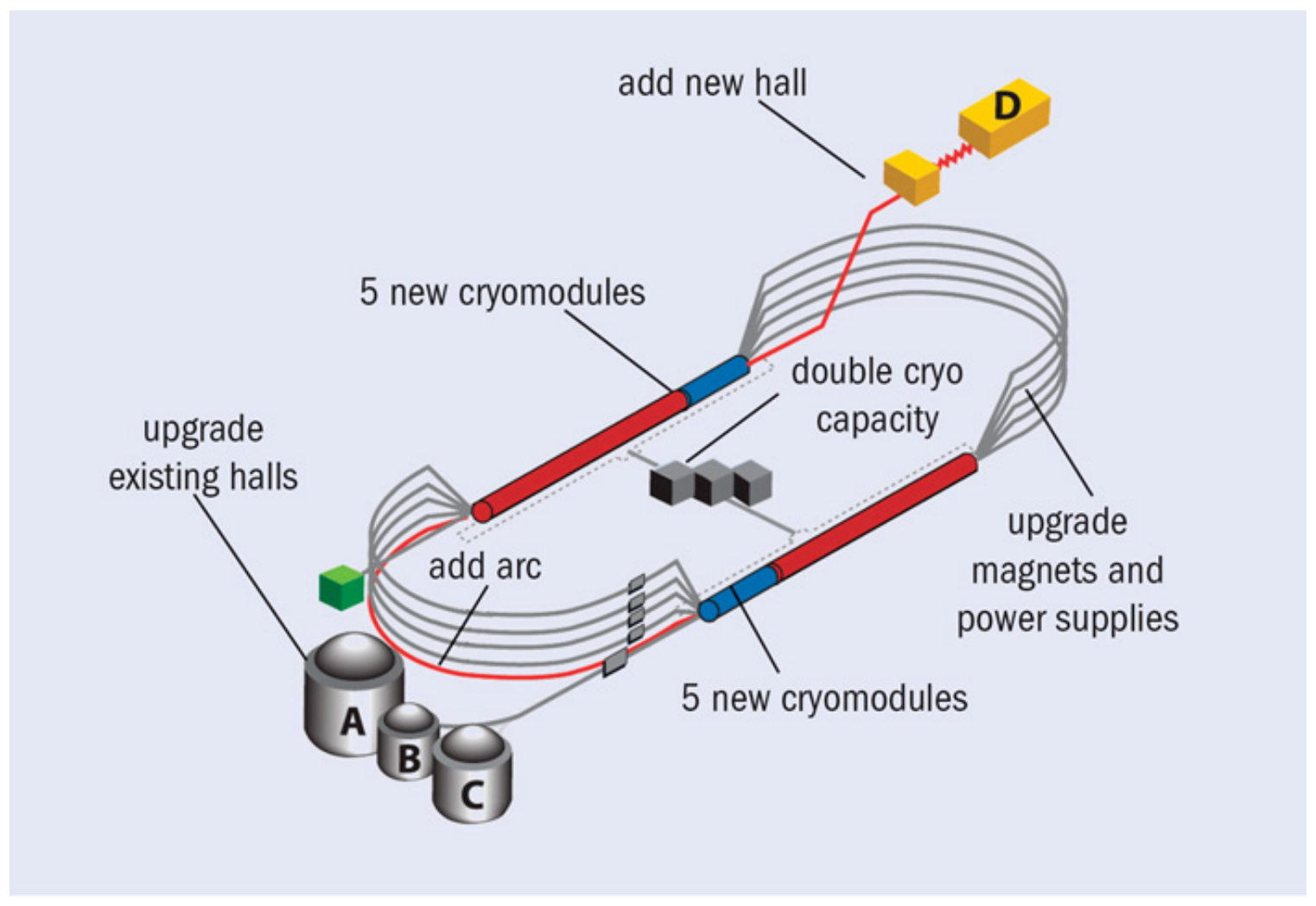

Figure 3.2: The Hall A end station at CEBAF. The electron beam is produced at the injector, accelerated through two linacs then extracted to the experimental halls.

The electron beam is produced at the injector then accelerated to $45 \mathrm{MeV}$. The beam is further accelerated by recirculating the beam up to 5 times through two superconducting linacs in the $12 \mathrm{GeV}$ design. Each pass increases the beam energy by $1200 \mathrm{MeV}$. The beam can be extracted to four different experimental halls simul- 
taneously and the beam current to each hall can be controlled independently.

\subsection{Beam Line}

The basic beam line components in Hall A [60 for E08014 are shown schematically in Figure 3.3. The function of each component will be described in the following sections.

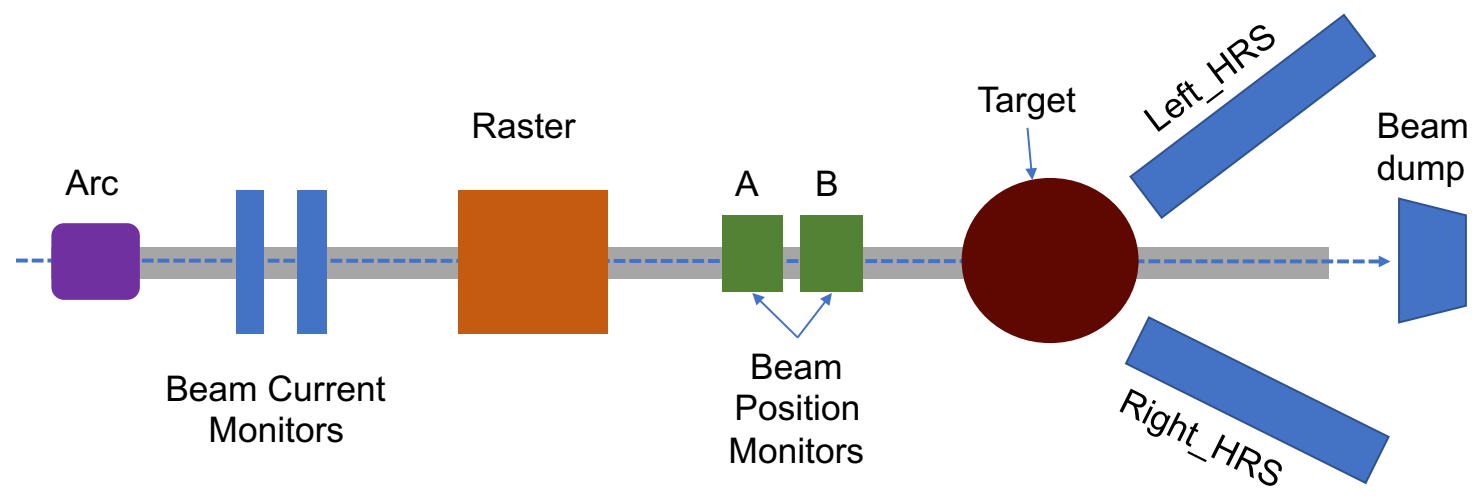

Figure 3.3: Hall A Beam line layout for Experiment E08-014

\subsubsection{Beam Position Monitors}

In Hall A, Beam Position Monitors (BPMs) are used to provide information of the beam position and angle at target $[61]$. The beam position is an important parameter for optics calibration and acceptance studies of the spectrometers. There are two BPMs, A and B, located at $7.345 \mathrm{~m}$ and $2.214 \mathrm{~m}$ upstream of the target, see Figure 3.3. Each BPM is comprised of 4 antennae arranged at $90^{\circ}$ to one other, oriented $\pm 45^{\circ}$ to the horizontal. They are used to extract the relative position of the beam to within $100 \mu \mathrm{m}$ for currents above $1 \mu \mathrm{A}$. The signal produced in the antennae by the beam passing through the BPMs is inversely proportional to the distance from the beam. The absolute position of the beam can be determined by calibrating them with 
respect to wire scanners called "harps", located adjacent to the BPMs, see Figure 3.4.

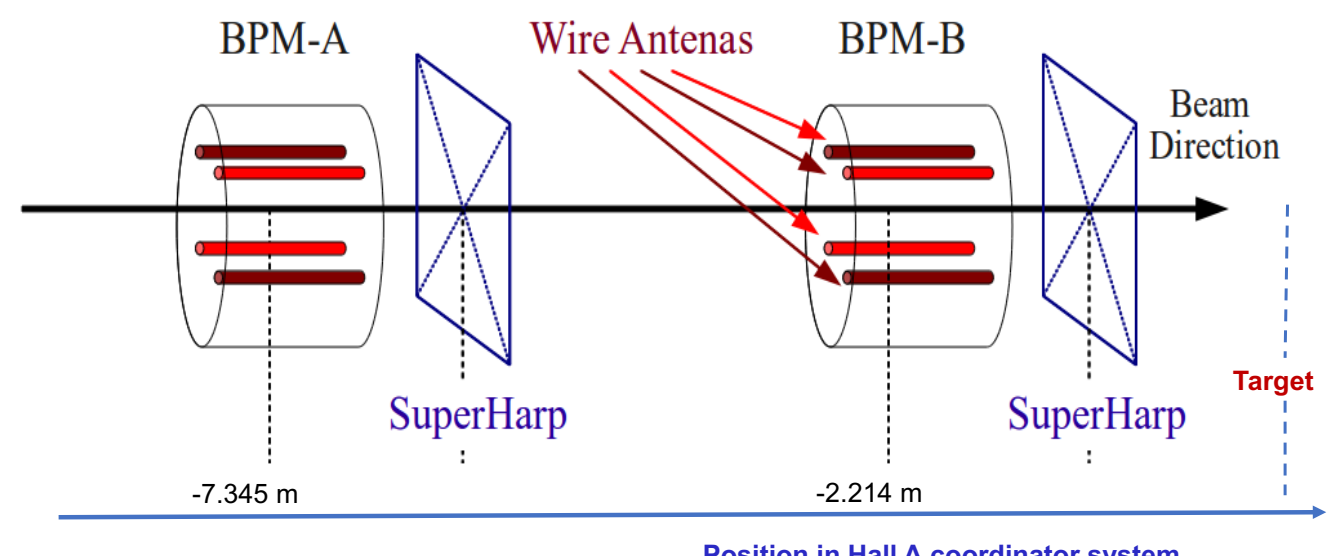

Position in Hall A coordinator system

Figure 3.4: Hall A BPMs and Harp schematic, see text.

\subsubsection{Raster}

The inherent beam size at CEBAF is small (on order of $100 \mu \mathrm{m}$ ) and with the high beam current used in this experiment $(40 \mu \mathrm{A}-100 \mu \mathrm{A})$, local boiling of cryogenic targets can occur. This is due to the deposited energy from the beam, and results in a non-uniform target density. To reduce this effect, the beam was rastered, increasing the effective spot size. The raster system is installed $23 \mathrm{~m}$ upstream of the target and consists of horizontal (X) and vertical (Y) dipole magnets. The dipole magnetic field is driven with frequency close to $25 \mathrm{kHz}$ by a triangular waveform. There is a small difference in the frequency of raster $\mathrm{X}$ and raster $\mathrm{Y}$ to avoid dwelling Lissajous curves. In addition, the rasters can be read out much faster than BPMs (due to the transmission time of the signal) which allows the beam position to be recorded event by event while BPMs provide only average beam position information. 


\subsubsection{Beam Current Monitor (BCMs)}

Most Hall A experiments need to measure the beam current with absolute accuracy of about $1 \%$. The CEBAF accelerator delivers continuous wave (CW) electron beams making precise current measurement more difficult than at a pulsed machine. The beam current monitors (BCMs) are designed for a stable, low-noise, non-interfering beam current measurement. It consists of an Unser monitor, and two RF cavities referenced as Up and Down (see Figure 3.5) and are installed in a temperaturecontrolled housing $25 \mathrm{~m}$ upstream of the target. The Unser monitor is a parametric current transformer which provides an absolute reference since it can be calibrated with a precision wire current. The two stainless steel, cylindrical RF cavities are on either side of the Unser and have a $Q \sim 500$.

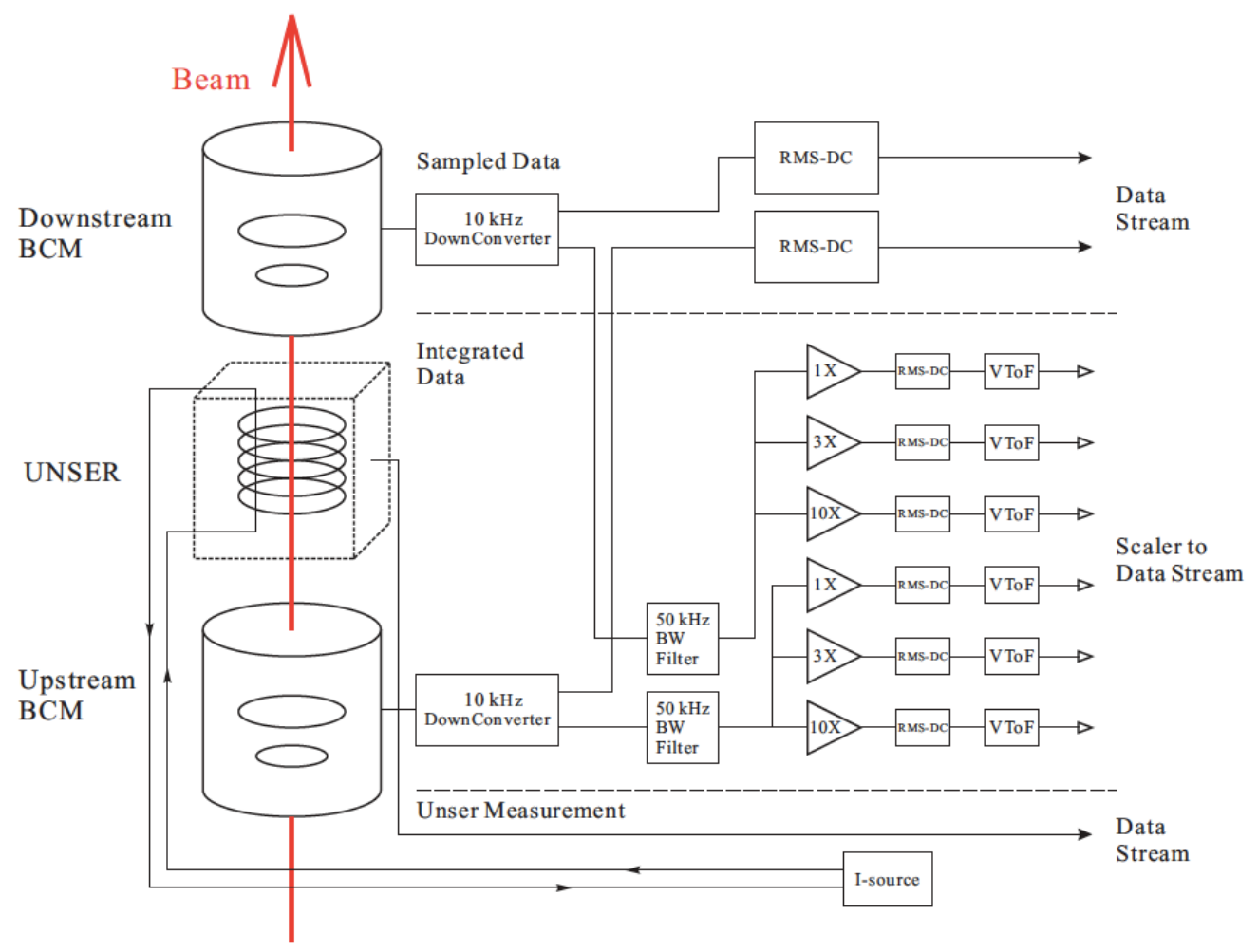

Figure 3.5: Hall A BCMs schematic including the Unser, up(down) BCMs and how the signal from these devices connected to scales and transfer to Data stream. 


\subsection{Target Setup}

There are three target loops in the cryogenic target system. Loop 1 and Loop 2 each have two aluminum cylindrical target cells mounted to a target ladder, with the lengths of $10 \mathrm{~cm}$ and $20 \mathrm{~cm}$, respectively while the Loop 3 has two $20 \mathrm{~cm}$ long cylindrical cells, also made of aluminum. The target assembly is installed inside the scattering vacuum chamber and includes the cryogenic target system with cooling, gas handling, temperature and pressure monitoring, target controller, and a solid target ladder.

This experiment ran in two time periods and we had two different target configurations [62], see Table 3.2. For period one $(04 / 11 / 2011-04 / 19 / 2011)$, the $20 \mathrm{~cm}$ loop 1 cell was filled with ${ }^{4} \mathrm{He}$ and the Loop 2 was filled with liquid deuterium (LD2), while Loop 3 was kept as a spare. For the second period (04/21/2011 - 05/15/2011), Loop 2 did not change but Loop 1 was filled with ${ }^{3} \mathrm{He}$. The solid targets ${ }^{40} \mathrm{Ca},{ }^{48} \mathrm{Ca}$ were installed in the former Loop 3 location (see Figure 3.6, Ref. 63, 64. Since the doubly magic ${ }^{48} \mathrm{Ca}$ is very expensive, it was installed in an aluminum target cell to protect it. ${ }^{40} \mathrm{Ca}$ was treated similarly to have consistent the aluminum backgrounds.

Table 3.2: The Cryogenic Target configuration for the two run periods 62

\begin{tabular}{||c|c|c||}
\hline \hline Target Loop & $\begin{array}{c}\text { Run Period 1 } \\
(04 / 11 / 11-04 / 19 / 11)\end{array}$ & $\begin{array}{c}\text { Run Period 2 } \\
(04 / 21 / 11-05 / 15 / 11)\end{array}$ \\
\hline Loop1 $20 \mathrm{~cm}$ cell & ${ }^{4} \mathrm{He}$ & ${ }^{3} \mathrm{He}$ \\
\hline Loop2 $20 \mathrm{~cm}$ cell & LD2 & LD2 \\
\hline "Loop3" $20 \mathrm{~cm}$ cells & Spare & ${ }^{40} \mathrm{Ca}$ and ${ }^{48} \mathrm{Ca}$ \\
\hline
\end{tabular}

The operating pressure and temperature for cryogenic targets are provided in Table 3.3 and Table 3.4. The position of each target on the target ladder can be found in Table 3.5. The window thickness of the cryogenic targets is listed in Table 3.6 [62]. Other targets are available for calibration included a Dummy target, an optics target and a single ${ }^{12} \mathrm{C}$ foil. For optics calibration we used 7 carbon foils cut 
Target configuration period 1

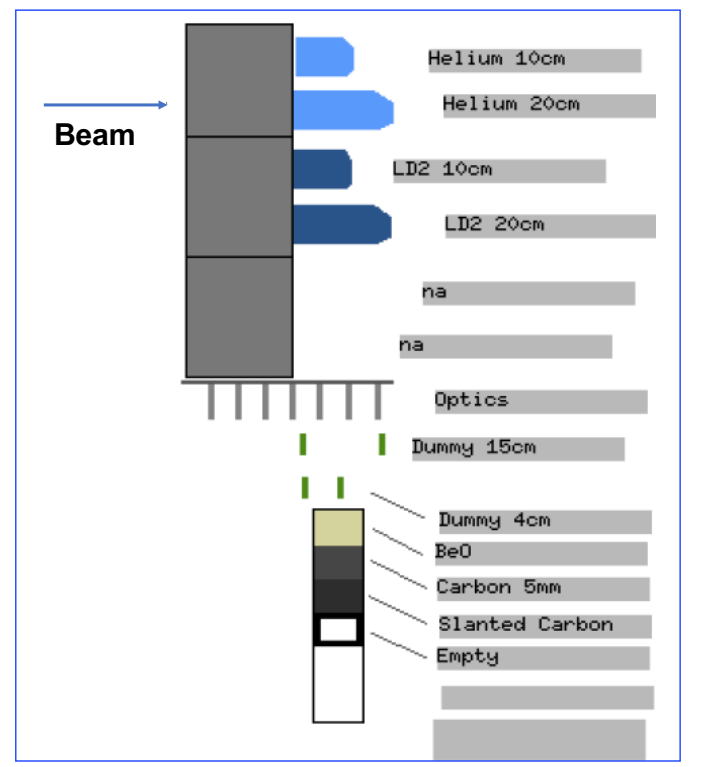

Target configuration period 2

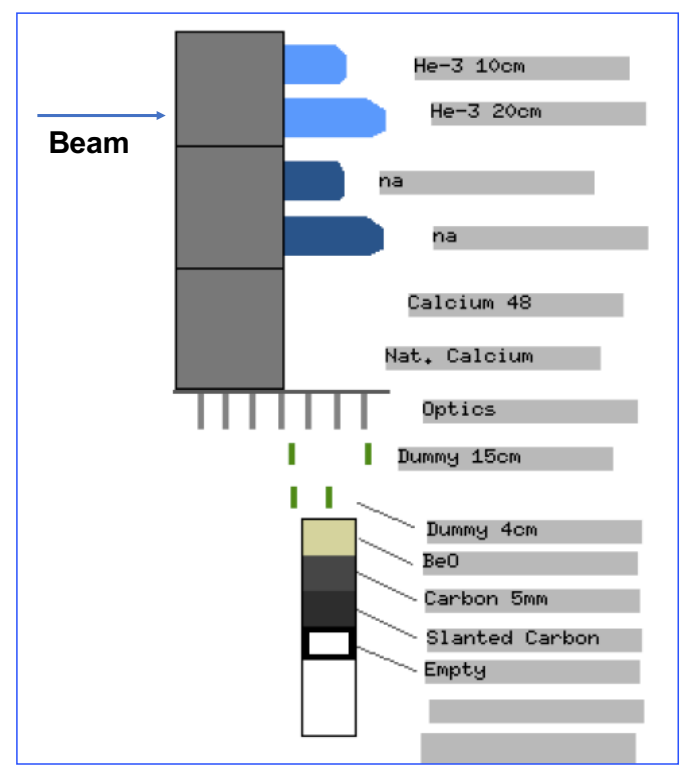

Figure 3.6: The target configuration for 2 run time periods. The left plot is target configuration for run period 1 while the right plot is for the run period 2 Refs. [63] [64]

Table 3.3: The operating information for Cryogenic Targets, Period 1 62

\begin{tabular}{||c|c|c|c||}
\hline \hline Target & Material & Pressure (psia) & Temperature (K) \\
\hline Loop1 $20 \mathrm{~cm}$ cell & ${ }^{4} \mathrm{He}$ & 202 & 20 \\
\hline Loop2 $20 \mathrm{~cm}$ cell & LD2 & 30.5 & 22 \\
\hline
\end{tabular}

from the same sheet. The upstream face of each foil is located at $0 \mathrm{~cm}, \pm 5 \mathrm{~cm}, \pm 10$ $\mathrm{cm}$ and $\pm 15 \mathrm{~cm}$. The Dummy targets are used for endcap contamination studies, and consist of two thick aluminum foils located at $\pm 5 \mathrm{~cm}$ or $\pm 10 \mathrm{~cm}$ around the nominal center for $10 \mathrm{~cm}$ and $20 \mathrm{~cm}$ dummy targets, respectively. A BeO target, a single ${ }^{12} \mathrm{C}$ foil and an empty target were installed below the $10 \mathrm{~cm}$ dummy target. Detailed information about solid targets can be found in Table 3.7 . 
Table 3.4: The operating information for Cryogenic Targets, Period $2[62$

\begin{tabular}{||c|c|c|c||}
\hline \hline Target & Material & Pressure (psia) & Temperature (K) \\
\hline Loop1 $20 \mathrm{~cm}$ cell & ${ }^{3} \mathrm{He}$ & 211 & 17 \\
\hline Loop2 $20 \mathrm{~cm}$ cell & LD2 & 30.5 & 22 \\
\hline
\end{tabular}

Table 3.5: The encoder position of each target on the Target ladder 62

\begin{tabular}{||c|c|c||}
\hline Target & Material/Description & BDS Encoder \\
\hline \hline Loop1 $10 \mathrm{~cm}$ & He & 35185744 \\
Loop1 $20 \mathrm{~cm}$ & He & 31610960 \\
Loop2 $10 \mathrm{~cm}$ & LD2 & 28036176 \\
Loop2 $20 \mathrm{~cm}$ & LD2 & 24461392 \\
Loop3 Spacer & Ca48 & 20886608 \\
Loop3 Spacer & Ca40 & 17311824 \\
Optics & Carbon & 14397294 \\
Dummy 20 cm & Aluminum & 13365584 \\
Dummy 10 cm & Aluminum & 11739984 \\
BeO & BeO & 9179984 \\
Solid Carbon & Carbon (5mm) & 7554384 \\
Slanted Carbon & Carbon & 3035983 \\
Empty & N/A & -10000000 \\
\hline
\end{tabular}

Table 3.6: The window thickness information for Cell targets 62

\begin{tabular}{||c|c|c|c||}
\hline \hline Position & $\begin{array}{c}\text { Entrance Window } \\
\text { Thickness (in) }\end{array}$ & $\begin{array}{c}\text { Exit Window } \\
\text { Side Wall (in) }\end{array}$ & $\begin{array}{c}\text { Exit Window } \\
\text { Nose (in) }\end{array}$ \\
\hline Loop1 $10 \mathrm{~cm}$ & $0.0107 \pm 0.0002$ & $0.0132 \pm 0.0007$ & $0.0142 \pm 0.0005$ \\
Loop1 $20 \mathrm{~cm}$ & $0.0108 \pm 0.0001$ & $0.0129 \pm 0.0006$ & $0.0139 \pm 0.0005$ \\
Loop2 $10 \mathrm{~cm}$ & $0.0104 \pm 0.0001$ & $0.0126 \pm 0.0006$ & $0.0141 \pm 0.0002$ \\
Loop2 $20 \mathrm{~cm}$ & $0.0107 \pm 0.0001$ & $0.0129 \pm 0.0011$ & $0.0142 \pm 0.0002$ \\
Loop3 $20 \mathrm{~cm}$ & $0.0107 \pm 0.0001$ & $0.0129 \pm 0.001$ & $0.0141 \pm 0.0002$ \\
\hline \hline
\end{tabular}


Table 3.7: Solid Targets information

\begin{tabular}{||c|c|c|c||}
\hline \hline Target Name & Material & Purity & Thickness $\left(\mathrm{g} / \mathrm{cm}^{2}\right)$ \\
\hline BeO & BeO & $99.0 \%$ & $0.149 \pm 0.001$ \\
Carbon & $\mathrm{C} 12$ & $99.95 \%$ & $0.8918 \pm 0.0008$ \\
Slanted Carbon & $\mathrm{C} 12$ & $99.9 \%$ & $0.419 \pm 0.005$ \\
Optics (7 foils) & $\mathrm{C} 12$ & $99.5 \%$ & $0.0248 \pm 0.001$ \\
each foil & & & \\
Dummy 10 cm & & & \\
Upstream & Aluminum & $\mathrm{N} / \mathrm{A}$ & $0.275 \pm 0.0003$ \\
Downstream & Aluminum & & $0.270 \pm 0.003$ \\
Dummy 20 cm & & & \\
Upstream & Aluminum & $\mathrm{N} / \mathrm{A}$ & $0.427 \pm 0.0003$ \\
Downstream & Aluminum & & $0.429 \pm 0.003$ \\
Ca48 & Calcium 48 & $\mathrm{N} / \mathrm{A}$ & $0.779 \pm 0.005$ \\
Ca40 & Calcium 40 & N/A & $0.819 \pm 0.01$ \\
\hline \hline
\end{tabular}




\subsection{Spectrometers}

In Hall A, there are two identical High Resolution Spectrometers (HRS) [59], the Left and Right, where Left and Right are respect to the beam line direction. Each spectrometer is a combination of two parts, a magnetic particle transport system and a detector package. There are 3 quadrupoles $(\mathrm{Q})$ and a dipole (D) in the magnet system, see Figure 3.7. Each detector package includes Vertical Drift Chambers, a Gas Cherenkov detector, scintillator counters, and a lead glass Calorimeter. A summary of the characteristics of the HRS can be found in the Table 3.8 and the arrangement of detector packages can be seen in Figure 3.8. The following sections will discuss the functions and characteristics for each detector in detector package.

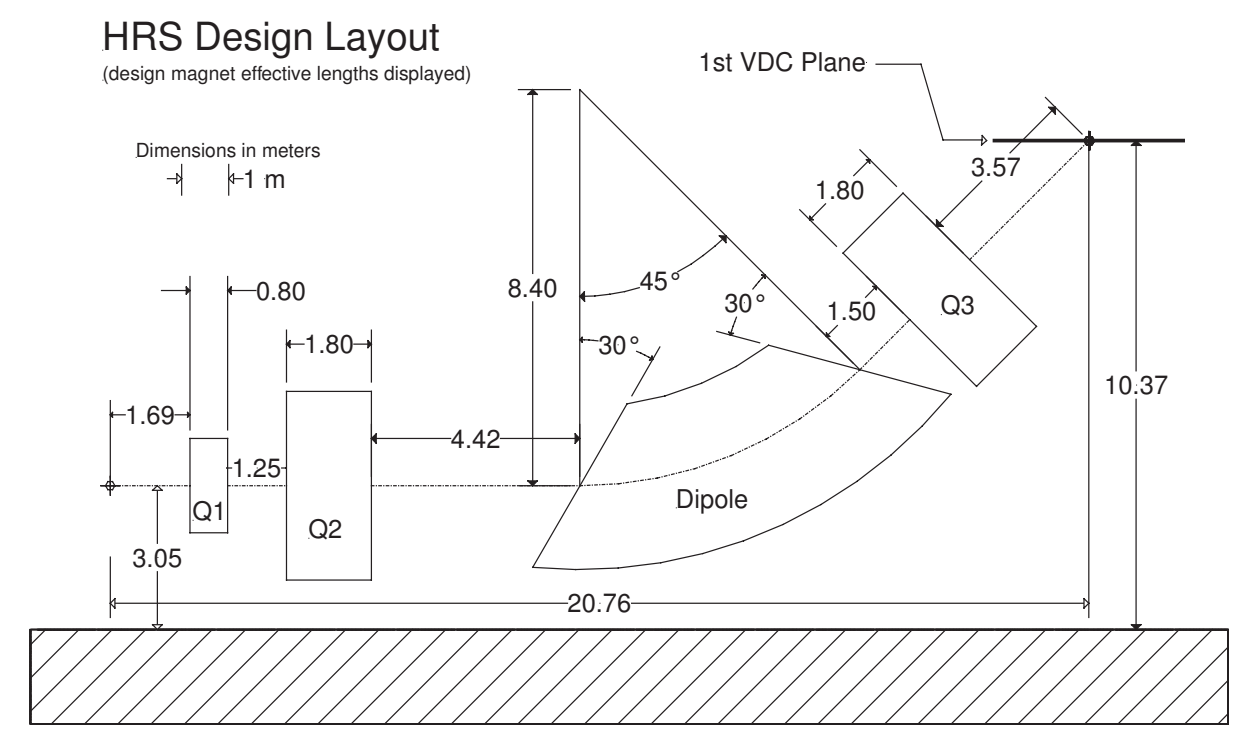

Figure 3.7: Hall A magnet system for HRS. The Q1 is convergent in the vertical plane. The Q2 and Q3 provide the transverse focusing. The Dipole is designed to have uniform field at the center for the bending angle [59].

\subsubsection{Vertical Drift Chambers}

Tracking information is provided by a pair of Vertical Drift Chamber (VDCs) in each HRS, described in detail in Refs. [59] and [65]. Each VDC is composed of two wire 

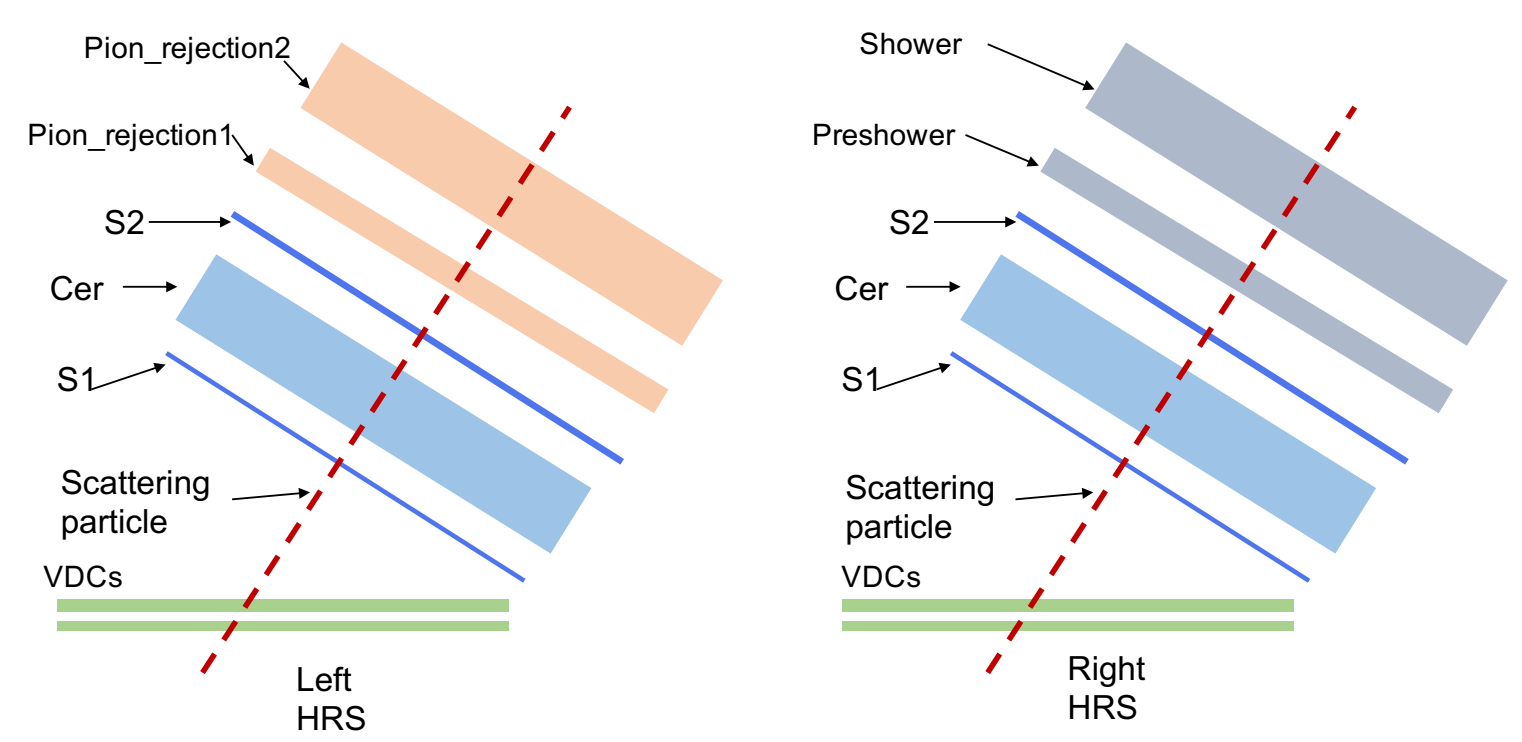

Figure 3.8: The Hall A HRS detector package. The left and right figures are for left and right HRS, respectively. Each of spectrometer includes the Vertical Drift Chambers (VDCs), scintillator counters (S1 and S2), a gas Cherenkov (Cer), and Calorimeters (For the left HRS they are called Pion-rejection1 and 2 (PR1, PR2). For the Right HRS they are called the Preshower and the Shower).

planes, separated by $3.35 \mathrm{~cm}$, in a standard UV configuration. The U and V planes are oriented $90^{\circ}$ to one another and lie in the horizontal plane of the hall, at an angle $45^{\circ}$ with respect to the dispersive and non-dispersive directions, see Figure 3.9. There are a total of 368 sense wires in each plane with a spacing of $4.24 \mathrm{~mm}$. The nominal particle trajectory crosses the wires planes at angle $45^{\circ}$.

The VDCs are filled with a mixture gas of argon (62\%) and ethane (38\%). The VDC electric field is shaped by gold plated Mylar planes, and generated by applying a high voltage of around $4 \mathrm{kV}$. The charged particles enter the VDCs, collide with gas molecules creating electron-ion pairs which drift along the electric field lines, and rapidly accelerate toward the closest wire. This acceleration generates many secondary electrons resulting in a signal which is ampliffed and sent to a TDC card. On the average, electrons that travel at nominal angle $45^{\circ}$ with respect to VDC planes normally fire 4 - 6 wires per plane as shown in Figure 3.10. An important advantage 
Table 3.8: The characteristics of Hall A where the resolution values listed are FWHM. See Ref. 59

\begin{tabular}{|c||c||}
\hline \hline Configuration & QQDQ vertical bend \\
Bend Angle: & $45^{\circ}$ \\
Optical Length: & $23.4 \mathrm{~m}$ \\
Momentum Range: & $0.3-4.0 \mathrm{GeV} / \mathrm{c}$ \\
Momentum Acceptance: & $-4.5 \%<\delta p / p<+4.5 \%$ \\
Momentum Resolution: & $1 \times 10^{-4}$ \\
Angular Range LHRS & $12.5-150^{\circ}$ \\
Angular Range RHRS & $12.5-130^{\circ}$ \\
Angular Acceptance Horizontal & $\pm 30 \mathrm{mrad}(\mathrm{Horizontal})$ \\
Angular Acceptance Vertical & $\pm 60 \mathrm{mrad}$ (Vertical) \\
Angular Resolution Horizontal & $0.5 \mathrm{mrad}$ \\
Angular Resolution Vertical & $1.0 \mathrm{mrad}$ \\
Solid Angle at $\delta p / p=0, y_{0}=0$ & $6 \mathrm{msr}$ \\
Transverse Length Acceptance: & $\pm 5 \mathrm{~cm}$ \\
Transverse Position Resolution: & $1 \mathrm{~mm}$ \\
\hline \hline
\end{tabular}

of the two-chamber design is that it provides a long lever arm for the measurement of track angles, resulting in excellent angle tracking resolution [59].

\subsubsection{Scintillators}

The scintillators are used to form the trigger for the DAQ system as well as provide the timing information for each event. There are two Scintillators (S1 and S2) separated by a distance of about $2 \mathrm{~m}$ as shown in Figure 3.11. The $\mathrm{S} 1$ plane is composed of 6 identical overlapping paddles made of thin plastic scintillator, while the S2 plane is made up of 16 paddles of a thicker scintillator. Each scintillator paddle is viewed by two PMTs, one at each end. When charged particles cross the scintillator paddles, they create the light that will be detected by both PMTs then converted to an analog signal. The average timing between two PMTs is called the mean time of a paddle. The difference of S1 and S2 mean times provides the time of flight for each particle. The time resolution per plane is approximately $0.30 \mathrm{~ns}$ [59] 

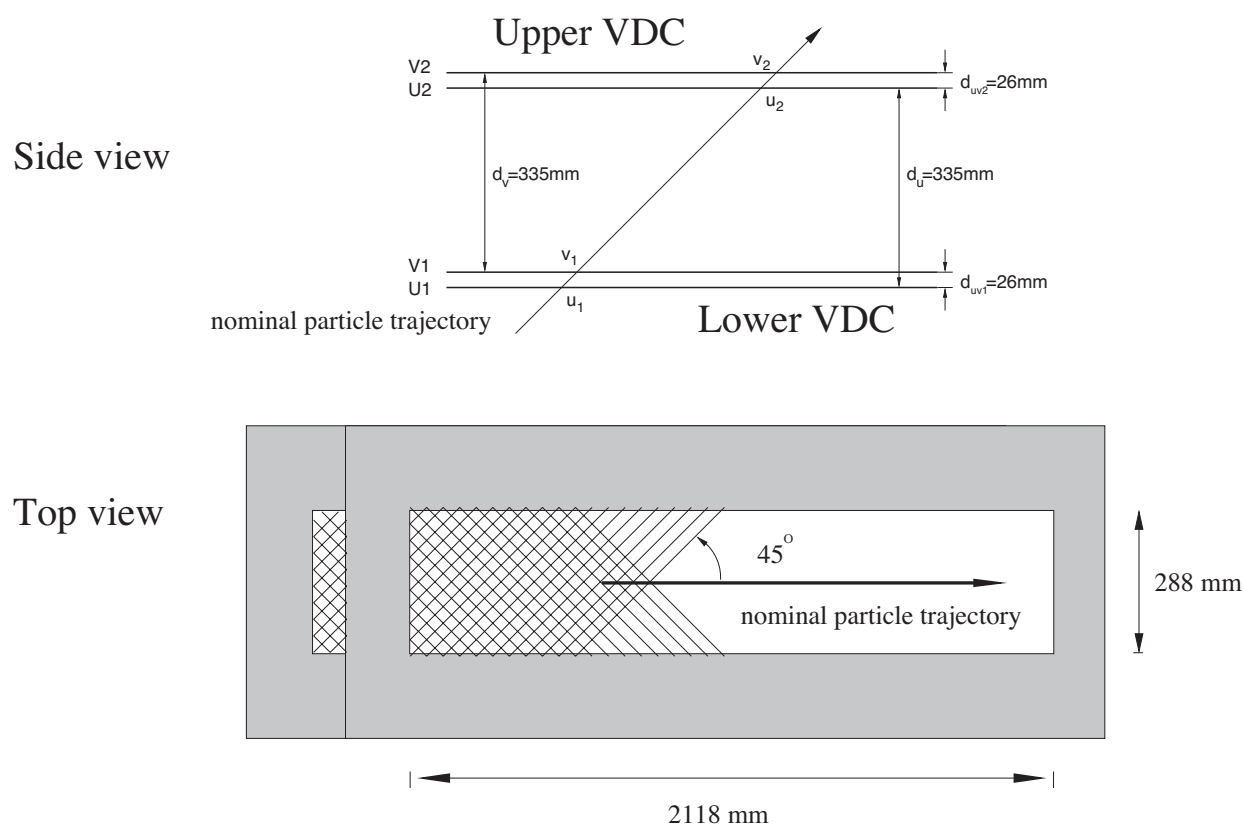

Figure 3.9: The lay-out of a pair of Vertical Drift Chamber for one HRS. The top plot, each VDC consists of one $\mathrm{U}$ and one $\mathrm{V}$ wire plane. The bottom plot, the VDCs are inclined at angle $45^{\circ}$ with respect to the dispersive and non-dispersive planes.

\subsubsection{Gas Cherenkov}

The Gas Cherenkov is responsible, along with the calorimeters, for particle identification in the HRS detector package. The Cherenkov is located between scintillators S1 and S2. The working principle of the Gas Cherenkov is that when a charged particle moves faster than the speed of light in a medium, it emits a small number of photons at a fixed angle $\theta$ determined by the velocity of particle $v(\beta=v / c)$ and the index of refraction of the medium $n$,

$$
\beta \geq \frac{1}{n} \Rightarrow \cos (\theta)=\frac{1}{\beta n} .
$$




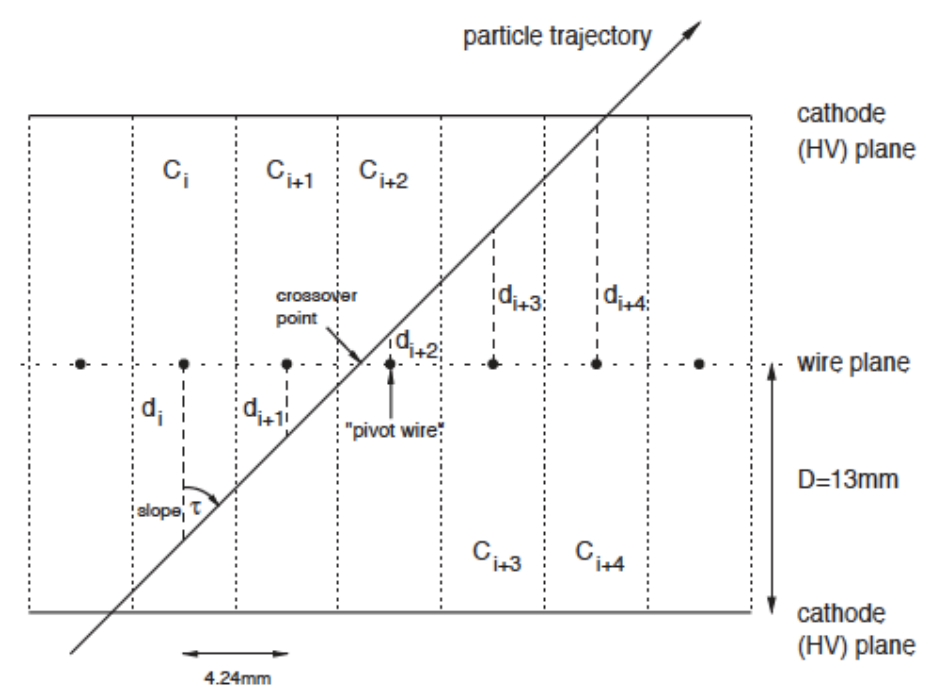

Figure 3.10: Showing a normal track fires 5 wires. The trajectory of a hypothetical particle as it fires 5 wires [59].

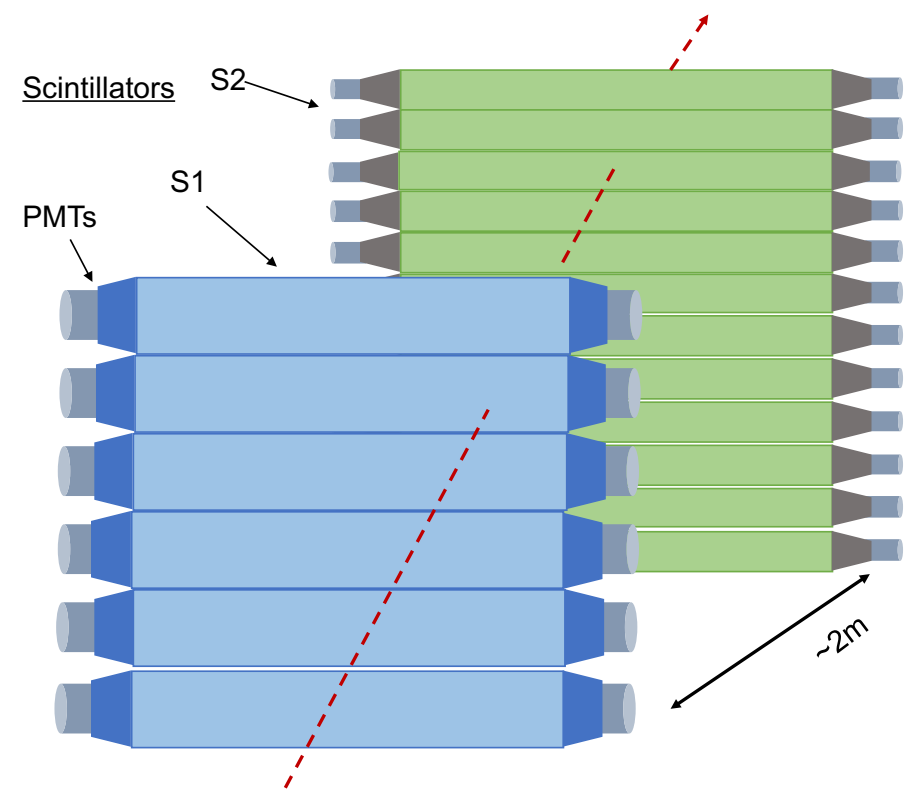

Figure 3.11: Hall A Scintillator Diagram

The momentum threshold for the production of Cherenkov radiation is based on this velocity threshold for different particles with different mass, as here

$$
p_{\text {threshold }}=\frac{m c}{\sqrt{\left(n^{2}-1\right)}}
$$


In this experiment, the Cherenkov detectors were filled with $\mathrm{CO}_{2}$ at atmosphere

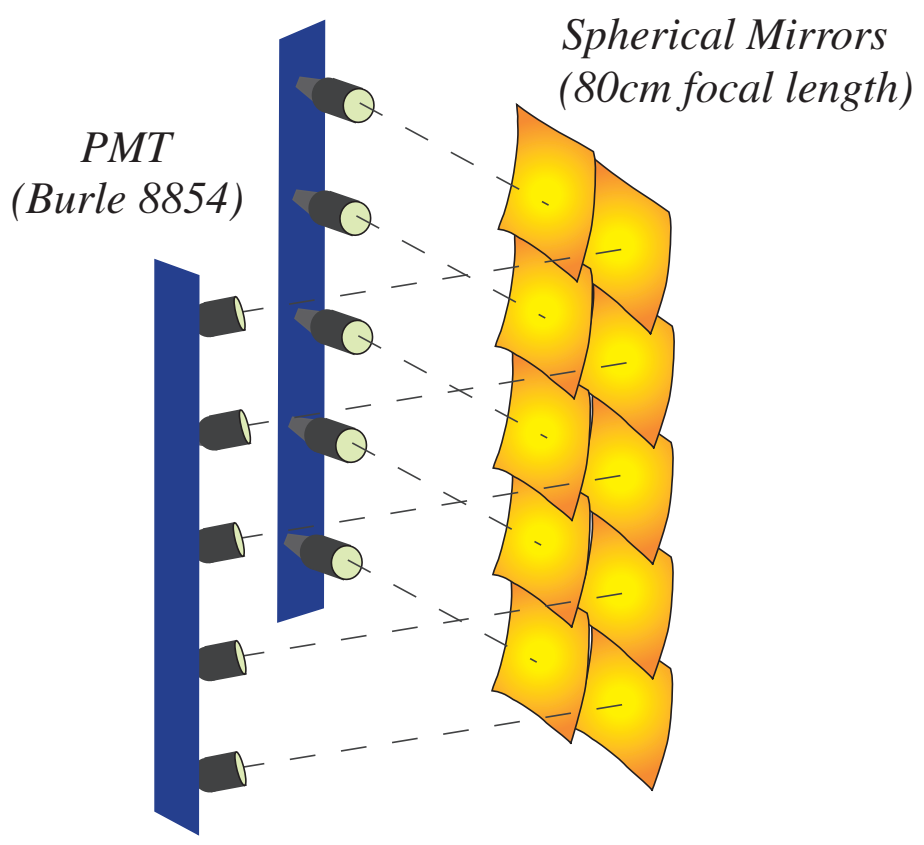

Figure 3.12: A Diagram of the Gas Cherenkov showing the spherical mirrors focusing the Cherenkov light to 10 PMTs.

pressure, which has an index of refraction of $n=1.00041$. For $\mathrm{CO}_{2}$, the momentum threshold for electrons is $0.017 \mathrm{GeV} / \mathrm{c}$, whereas the threshold for pions is $4.8 \mathrm{GeV} / \mathrm{c}$, well above the maximum momentum of HRS. In this way the detection of Cherenkov light is a very effective way for electron/pion discrimination, since only electrons create a significant signal. Once the Cherenkov light is produced, it is detected by 10 PMTs, (see Figure 3.12), installed on both sides of the detector. The signals from 10 PMTs are summed together, and used for particle identification cuts in data analysis.

\subsubsection{Calorimeters}

The calorimeters provide further electron/pion discrimination by measuring the energy deposited by particles that pass through it. An accelerating high energy electron emits photons through Bremsstrahlung. These photons undergoes pair production, producing electron-positron pairs, and the process is repeated such that the number 
of charged particles increases exponentially with the depth in the calorimeter. This process gives the calorimeter another name, a shower detector.

Electrons and positrons deposit almost all of their energy in the calorimeter but pions deposit a smaller amount of energy due to direct Cherenkov light and occasional $\pi^{0}$ production, etc. If $\mathrm{E} / \mathrm{p} \sim 1$ then the particle is an electron ( $\mathrm{E}$ is deposited energy in calorimeters and $\mathrm{p}$ is particle momentum).

\section{HRS-L}
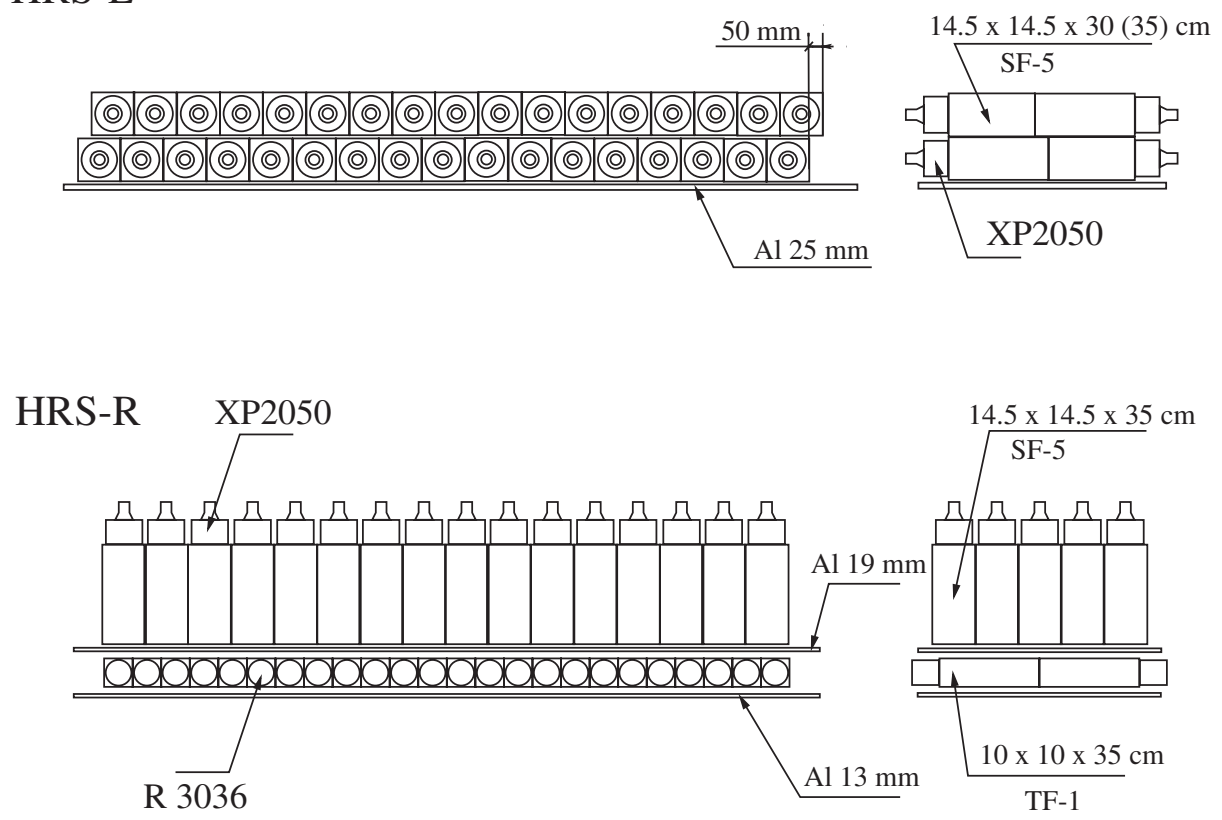

Figure 3.13: Schematic lay-out of part of the shower detector in HRSL and HRSR, see Ref. 59

The configuration of calorimeters in HRS-L and HRS-R are a bit different. For HRS-L, the calorimeter is a combination of two layers made of 34 lead-glass blocks, called pion rejector 1 and 2 (PR1, PR2). Both layers are oriented perpendicular to the particle trajectory. The dimension of blocks in these two layers are $15 \mathrm{~cm} \mathrm{x}$ $15 \mathrm{~cm}$ x $30 \mathrm{~cm}$. The HRS-R calorimeter also has two layers of lead-glass blocks, called the Preshower and the Shower. The Preshower is comprised of 48 lead-glass blocks, oriented perpendicular to the particle trajectory and each block is $10 \mathrm{~cm} \mathrm{x} 10 \mathrm{~cm} \mathrm{x}$ $35 \mathrm{~cm}$. The shower is made of 80 lead-glass blocks, oriented parallel to the particle 
trajectory and each block is $15 \mathrm{~cm} \times 15 \mathrm{~cm} \times 35 \mathrm{~cm}$. The calorimeter in the LHRS is not a full energy deposition calorimeter, while the RHRS is a total energy absorber. The light produced by the cascade is linearly proportional to the energy deposited and is collected by PMTs mounted to each lead-glass block.

\subsubsection{Trigger Design}

Three detectors were used to build the trigger for DAQ system for E08014 and they are S1, S2 and the Gas Cherenkov.

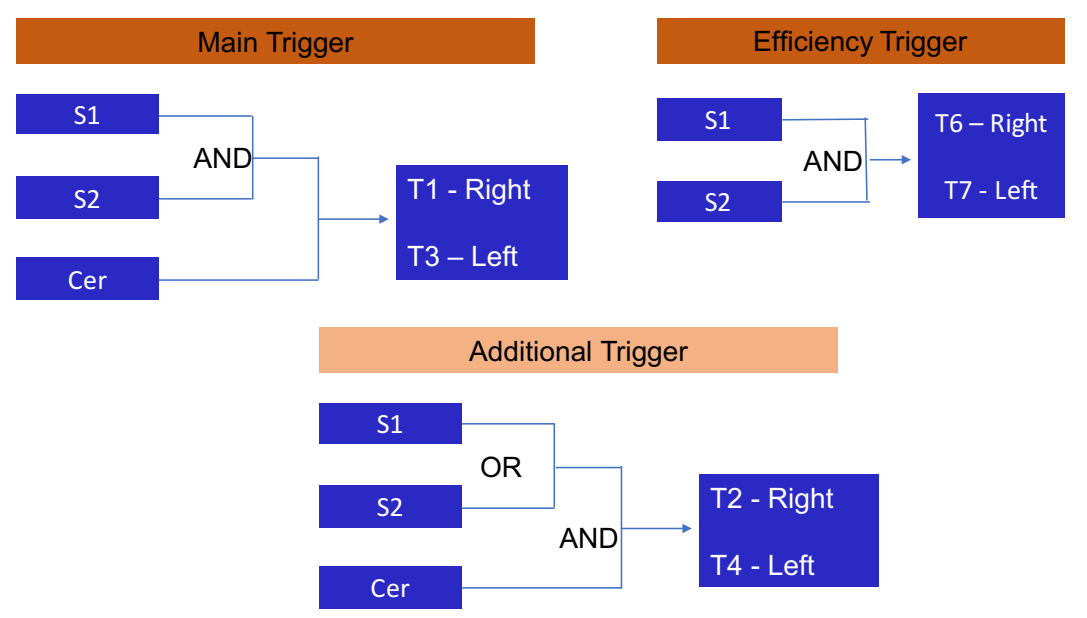

Figure 3.14: Trigger Design for the Experiment E08014

For the production data we used the main trigger consisting of the coincidence signal of S1, S2 and Gas Cherenkov. To be able to check the efficiency of the main trigger, another trigger was built using coincidence of S1 and S2. We have additional triggers in this experiment which are coincidence of either S1 OR S2 AND the Cherenkov. Figure 3.14 is the summarizes of how all triggers are designed. 


\section{Chapter 4}

\section{Data Analysis}

\subsection{Overview}

This chapter will discuss the calibrations of the beam line components and detectors. Some of the calibrations was done in a earlier analysis [54]. Here the focus is on the calibrations and the corrections necessary to improve the quality of the results. The optics calibrations and the acceptance studies will be discussed in this Chapter along with the beam charge and live time calculations and the efficiency studies of particle identification and tracking. The trigger efficiency will not be included here as it needs a special treatment that will be discussed in Chapter 5. The analysis presented here provides all needed information for extracting the cross section.

\subsection{Beamline Calibration}

\subsubsection{BPM calibration}

The BPM calibration provides the parameters to convert the BPMs signals to beam positions, necessary to get the vertex information for each event. The results of the calibration from the previous analysis was the starting point. But during this 
analysis, it was determined that the sign of the BPM position in the $\mathrm{X}$ direction was flipped while the sign of the BPM position in the Y direction was correct. The Hall A coordinate system and accelerator coordinate system (MCC) are left handed and right handed, respectively, see Figure 4.1 [66. It means that the BPM information in the root files, which uses the Hall A coordinate system and BPM information from MCC which uses the accelerator coordinate system, must have opposite signs in the $\mathrm{X}$ direction. We can use what is called Bulls-eye data listed in table 4.1 to confirm the convention of coordinates and then confirm that the BPM in X direction was flipped.

\begin{tabular}{||c|c|c|c|c|c||}
\hline \hline Run number & $\begin{array}{c}\text { BPMA-X } \\
(\mathrm{mm})\end{array}$ & $\begin{array}{c}\text { BPMA-Y } \\
(\mathrm{mm})\end{array}$ & $\begin{array}{c}\text { BPMB-X } \\
(\mathrm{mm})\end{array}$ & $\begin{array}{c}\text { BPMB-Y } \\
(\mathrm{mm})\end{array}$ & movement (MCCs) \\
\hline 3877 & 0.7 & 1.673 & -1.49 & 2.988 & $+1 \mathrm{~mm}$ in $\mathrm{Y}$ \\
\hline 3878 & 0.667 & -1.058 & -1.51 & 0.99 & $-1 \mathrm{~mm}$ in $\mathrm{Y}$ \\
\hline 3880 & 1.482 & 0.33 & -0.499 & 2.003 & $+1 \mathrm{~mm}$ in X \\
\hline 3881 & -0.117 & 0.294 & -2.499 & 2.004 & $-1 \mathrm{~mm}$ in X \\
\hline 3882 & 0.631 & 0.308 & -1.512 & 1.99 & Nominal position \\
\hline
\end{tabular}

Table 4.1: Bulls-eye Scan Data includes 5 runs, for each run the beam position is moved from the nominal position to left or right, up or down, then back to nominal. The beam position information at each location is recorded in both Hall A and accelerator coordinate.

Using the beam X convention in the Hall A coordinate system (see Figure 4.2), if beam moves to positive $\mathrm{X}$ direction then $\mathrm{Y}_{t g}$ will move in the positive direction as well. It means that $Y_{t g}$ of run 3880 should be more positive compared to the one from Run 3882 (as the beam moves $+1 \mathrm{~mm}$ in X direction in Run 3880 compare to Run 3882). But that is not what happened. As can be seen in Figure 4.3 which shows the reconstructed $\mathrm{Y}_{t g}$ distribution from data for these two Runs, it moved in the opposite direction. This confirmed that the X direction in Hall A and MCC were opposite. But the BPMs information in the root files had the same sign as MCC values. This is confirmation that the BPM in the $\mathrm{X}$ direction was flipped. 


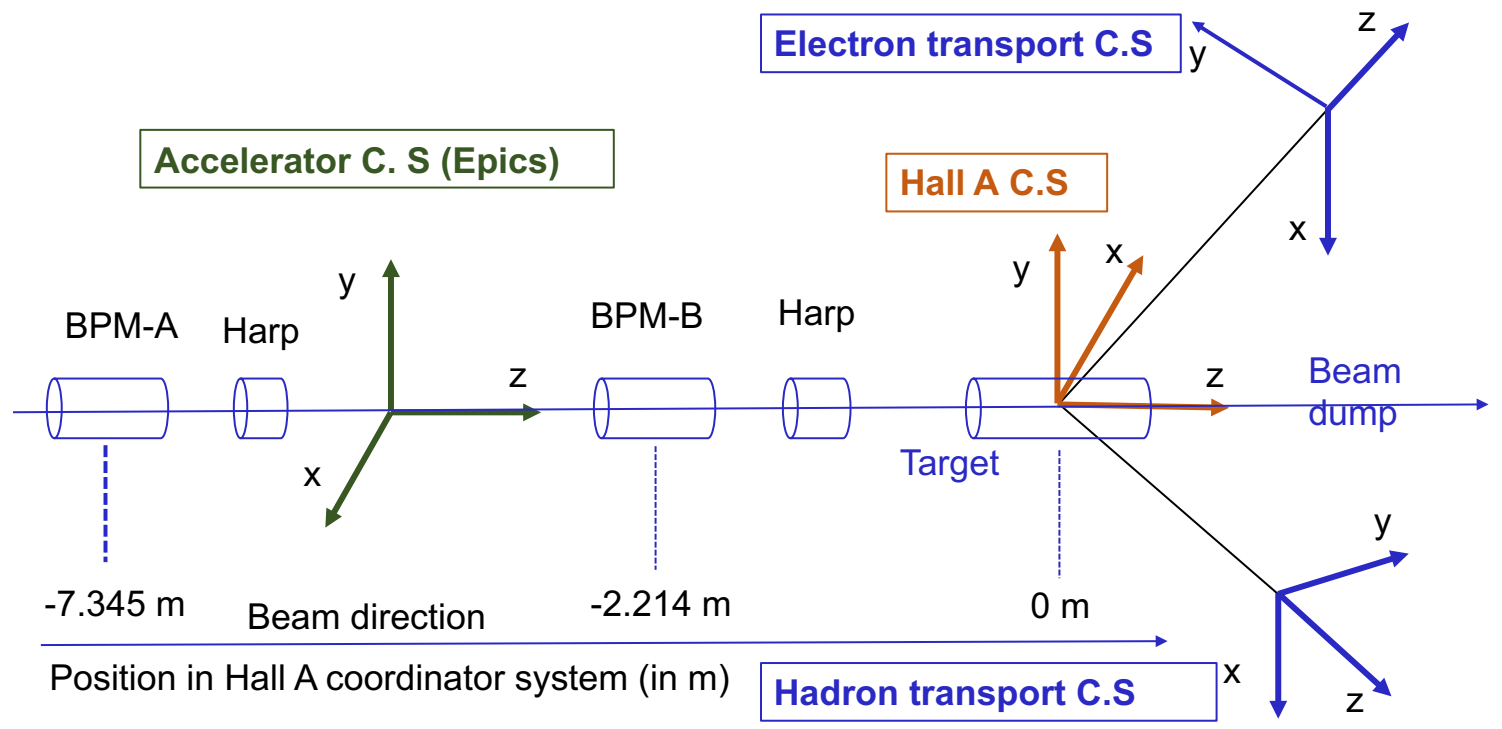

Figure 4.1: BPM in Hall A and accelerator coordinate system. The x-axes are in opposite directions. For details see Ref. [66].

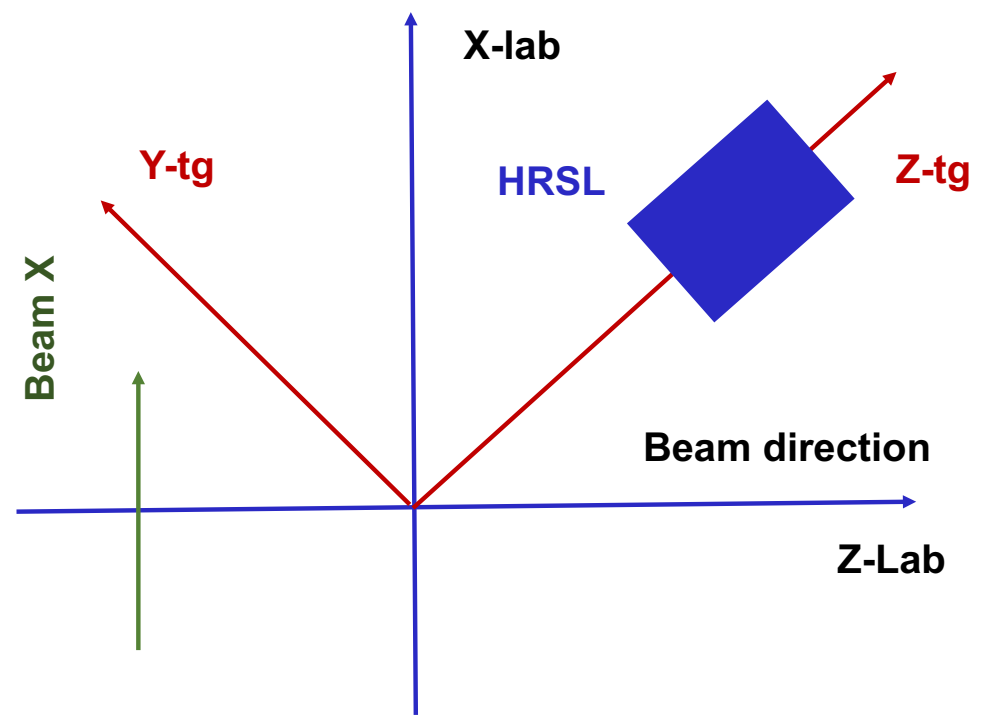

Figure 4.2: Beam X convention in the Hall A coordinate system

This problem needed to be fixed to provide the correct BPM information. It was not necessary to do new BPM calibration. In order to get beam information from the 


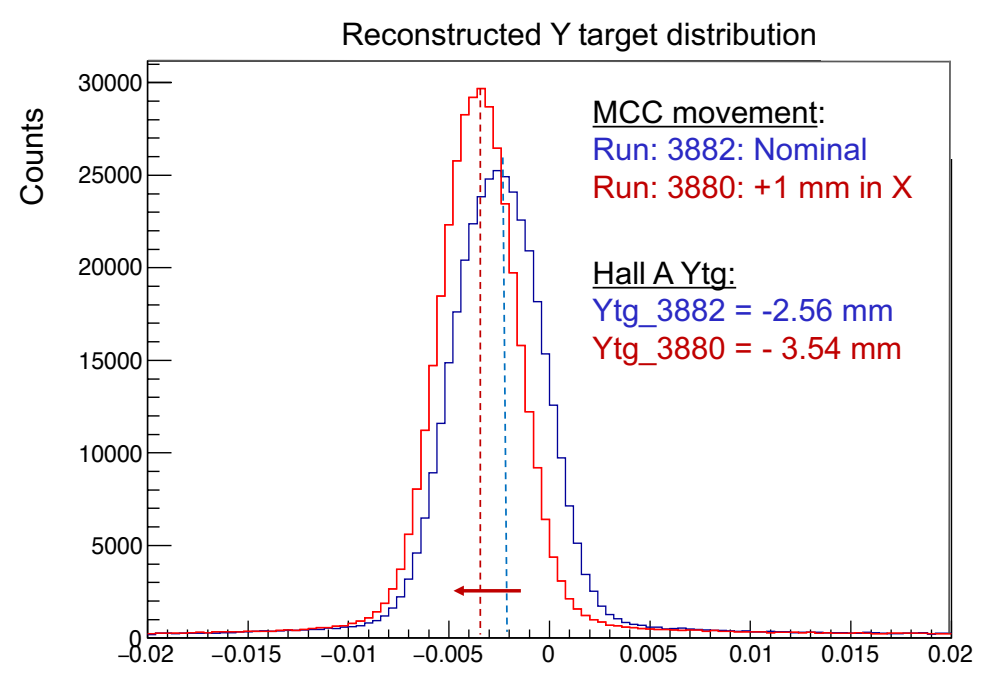

Figure 4.3: $\mathrm{Y}_{t g}$ distribution of Bull-eyes scan runs for beam $\mathrm{x}$ convention. The beam was moved $+1 \mathrm{~mm}$ in the Run 3880 compared to Run 3882 but the $\mathrm{Y}_{t g}$ distribution of Run 3880 is more negative compare to the one of Run 3882 what indicates the beam was moving in negative direction in Hall A coordinator system.

BPMs, one uses Equations 4.1 and 4.2 and a set of coefficients shown in Table 4.2 .

$$
\begin{gathered}
\left(\begin{array}{l}
\text { BeamX } \\
\text { BeamY }
\end{array}\right)=\left(\begin{array}{ll}
C 1 & C 2 \\
C 3 & C 4
\end{array}\right)\left(\begin{array}{l}
\operatorname{Rot} X \\
\operatorname{Rot} Y
\end{array}\right)+\left(\begin{array}{l}
C 5 \\
C 6
\end{array}\right) \\
\text { BeamX }=C 1 * \operatorname{Rot} X+C 2 * \operatorname{Rot} Y+C 5
\end{gathered}
$$

Table 4.2: BPMs coefficients from an early Calibration for E08-014, Ref. [54]

\begin{tabular}{|c|c|c|c|c|c|c||}
\hline \hline BMP & C1 & C2 & C3 & C4 & C5 & C6 \\
\hline A & 0.66899 & -0.684665 & 0.672353 & 0.683605 & 0.000147627 & -0.00063362 \\
\hline B & 0.659737 & -0.836241 & 0.646295 & 0.839493 & 0.00493647 & -0.00602042 \\
\hline
\end{tabular}

The RotX and RotY are the beam position in the rotated coordinate system of the 4 wire antennas, as in Figure 3.4 . The BPM calibration provide the $2 \times 2$ rotation matrix and offset terms to be able to convert the beam position from the rotated 
coordinate system to the lab coordinate system, BeamX and BeamY. Hence the sign of three coefficients C1, C2 and C5 must be flipped to fix the sign of BeamX.

Using the data with the raster off (only using the BPM information) and the flipped sign of coefficients, C1, C2, C5 it can be confirmed that the values agree well with MCC values and have the opposite sign. Upon examination of the event distribution as function of Z-vertex, determined from the BPM information, a large improvement can be seen. Previously there was a large unexplained offset in Z-vertex $(1 \mathrm{~cm})$ but after this fix the offset is about $1 \mathrm{~mm}$ which is not unreasonable for the target offset. See Figure 4.4 .

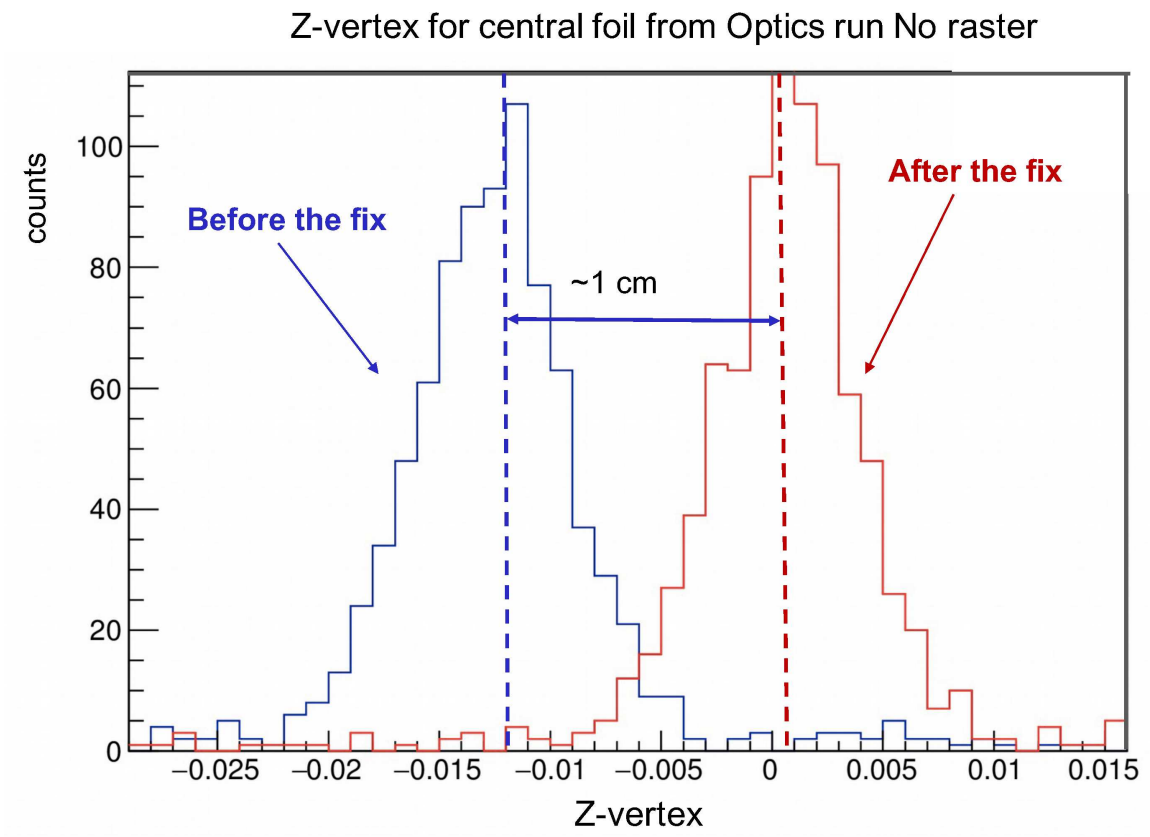

Figure 4.4: The Z-vertex distribution before and after fixing the BPMX. Before the fix, there is a large offset $(1 \mathrm{~cm})$ in the Z-vertex. After the fix the offset is around 1 mm.

The corrected average beam position now can be obtained using the updated BPM coefficients. In order to get the beam information for each event, a new raster calibration is required as it was based on the BPM's information. 


\subsubsection{Raster Calibration}

During production data taking, the beam raster was used to increase the beam spot size. Subsequently, the beam position information comes from the raster system. The raster calibration determines the parameters to convert the raster current to a beam position at the location of BPMA, BPMB and the target (see Figure 3.4). The BPM information and the raster current are both needed for the calibration to provide the separate parameters sets for each location. There is a phase lag between the actual beam position recorded by raster current and the one recorded by BPMs, see Figure 4.5. This phase lag is due to the fact that the raster current signal can be recorded instantaneously what gives the actual beam position at the moment but the BPMs signal arrives later due to transmission time, so when BPMs signal is recorded it reflects the beam position from few moment earlier. The BPMs are only used for the average position. The raster current is recorded for each event and used to get beam position event by event 66 68.

Figure 4.6 shows an event distribution for beam $\mathrm{X}$ at the target and raster current $\mathrm{X}$, it is similar for beam $\mathrm{Y}$ and raster current $\mathrm{Y}$. Recall that, we only measure the beam position at BPMA and BPMB. The BPM distribution at the target is the projection from the one BPMA and BPMB based on their distance to the target. The only tricky problem for the raster calibration is determining the direction beam

moves when the raster current changes. The sign of $K_{x}$ and $K_{y}$ in equation 4.3 must be determined.

$$
\begin{aligned}
& X_{\text {offset }}=t a_{x}-r a_{x} \cdot d t a_{x} / d r a_{x} \cdot K_{x} \\
& X_{\text {slope }}=d t a_{x} / d r a_{x} \cdot K_{x} \\
& Y_{\text {offset }}=t a_{y}-r a_{y} \cdot d t a_{y} / d r a_{y} \cdot K_{y} \\
& Y_{\text {slope }}=d t a_{y} / d r a_{y} \cdot K_{y},
\end{aligned}
$$



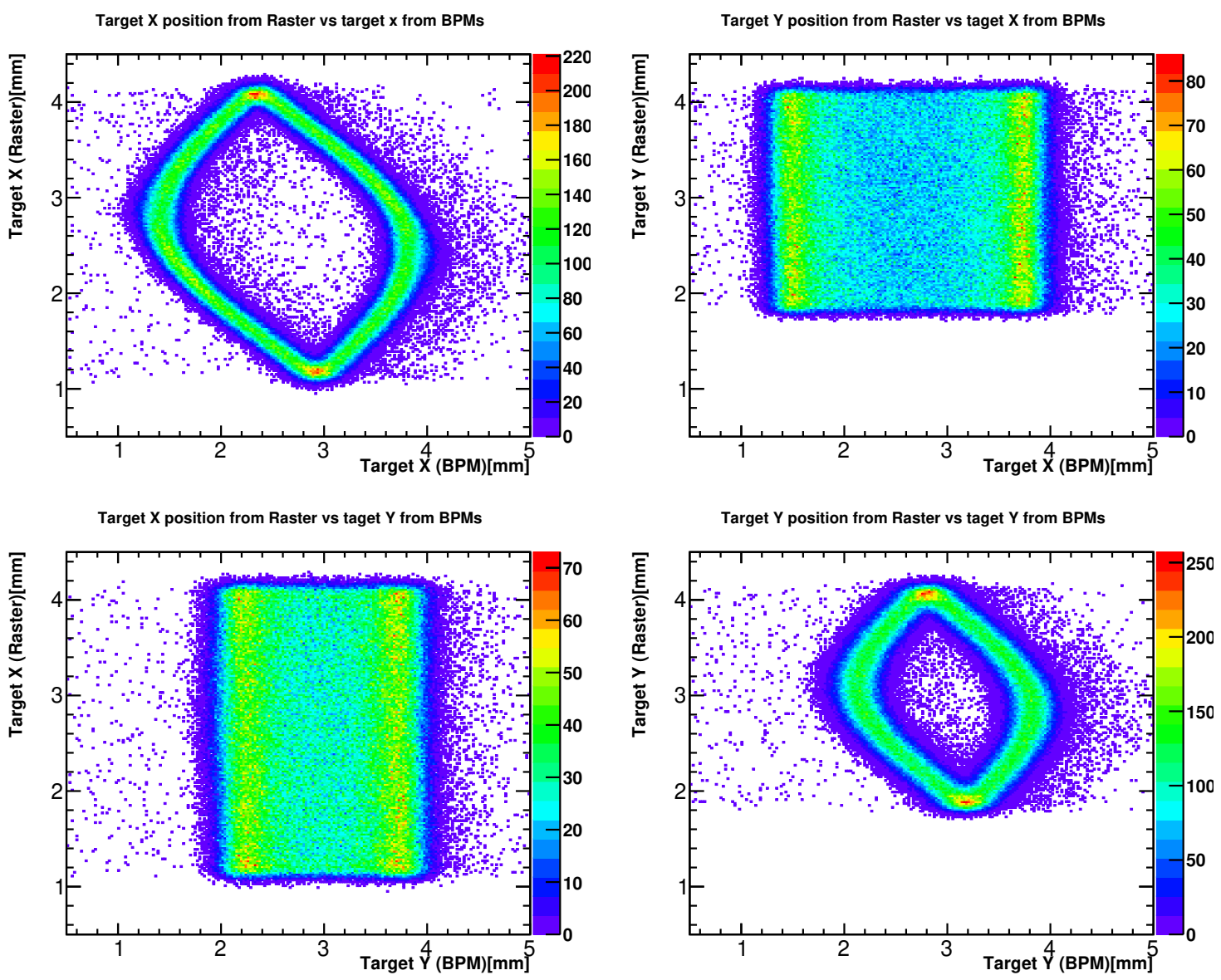

Figure 4.5: The phase lag in the beam position recorded by BPMs and from raster current. See the text for more information.
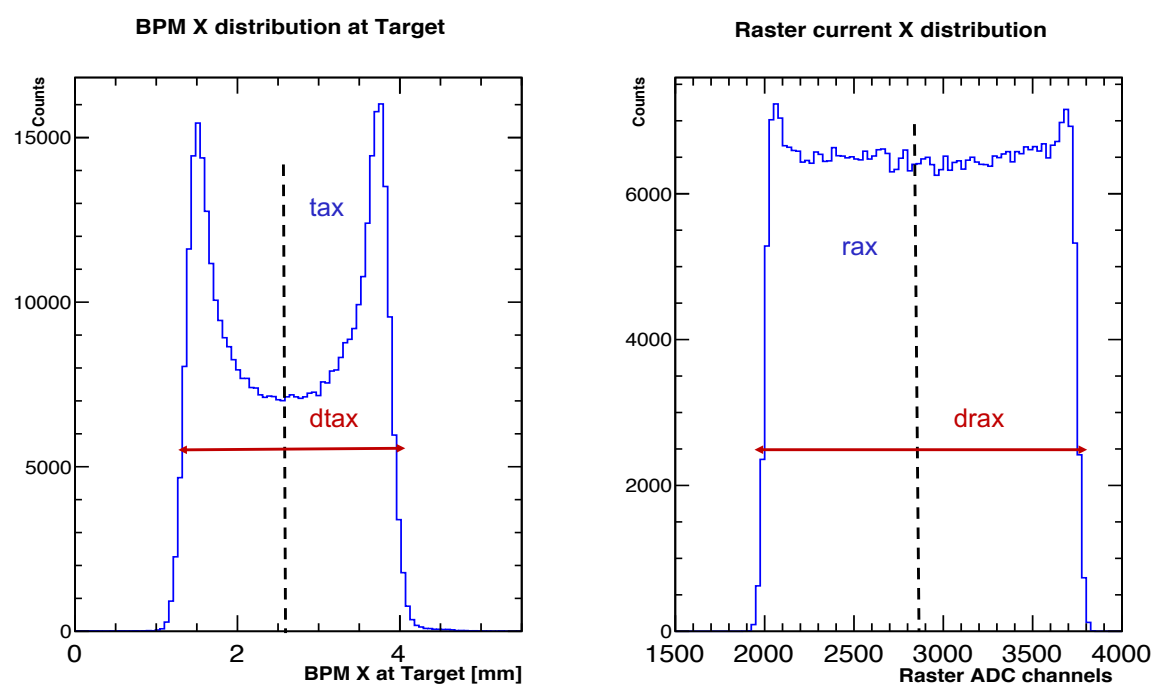

Figure 4.6: Raster Calibration at Target location 
where $t a_{x}, d t a_{x}, r a_{x}, d r a_{x}$ are the mean and the width of BPM X distribution at target and the raster current $\mathrm{X}$ distribution, respectively. The beam position can be calculated from the raster coefficient as follows:

$$
\begin{aligned}
& X_{\text {beam }}=X_{\text {slope }} \cdot X_{\text {curr }}+X_{\text {offset }} \\
& Y_{\text {beam }}=Y_{\text {slope }} \cdot Y_{\text {curr }}+Y_{\text {offset }}
\end{aligned}
$$

To be able to determine the sign of $K_{x}$ we can use the plot of raster current $\mathrm{X}$ versus the $\mathrm{Y}_{t g}$ as in Figure 4.7. When the raster current increases (i.e the ADC channel increases), $\mathrm{Y}_{t g}$ is more positive and this means that the position of the beam moves in the positive direction. The beam $\mathrm{X}$ position is proportional to the raster current $\mathrm{X}$ and $K_{x}=+1$.

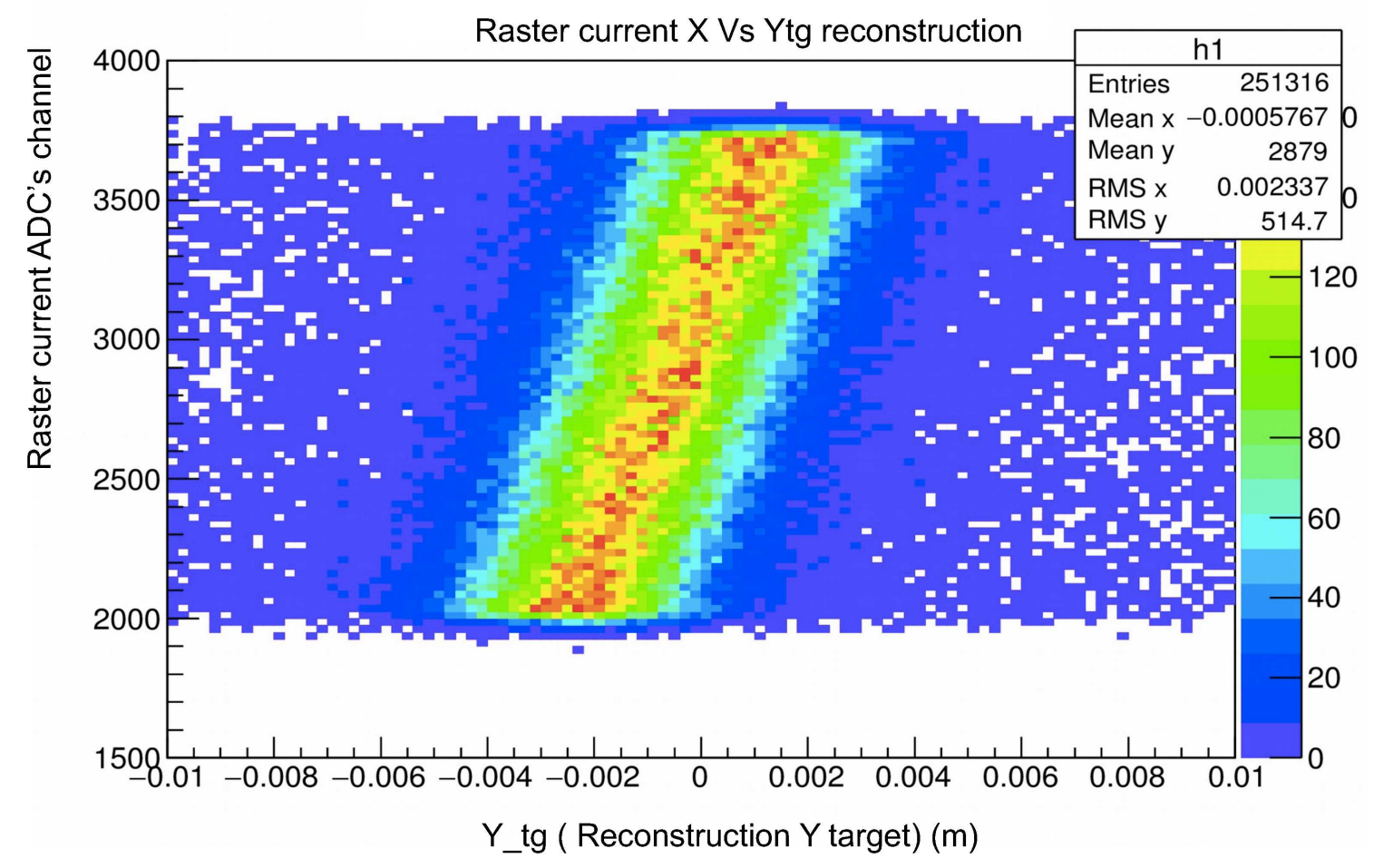

Figure 4.7: Determining the sign of $K_{x}$ for Raster Calibration using the raster current $\mathrm{X}$ vs the reconstructed $\mathrm{Y}_{t g}$. The $\mathrm{Y}_{t g}$ moves to positive direction when the raster $\mathrm{X}$ current's ADC channel increases. This indicates the beam $\mathrm{X}$ position is proportional to the raster current $\mathrm{X}$. 
It is more difficult to determine the sign of $K_{y}$. During E08-014 no specific data was taken for this determination. However optics data taken with a sieve can be used. The sieve is a thick plate with many small holes so that the particles going through the same hole are taken to have the same scattering angle and others are absorbed. If we select events from a specific hole with the raster on we are able to see the small variance in the out-of-plane angle $\left(\theta_{t g}\right)$. The first step in this work is to select the central foil from the multiple foils target data. Then we select the central hole from the sieve pattern of the central foil. See Figure 4.9. In the end we will see

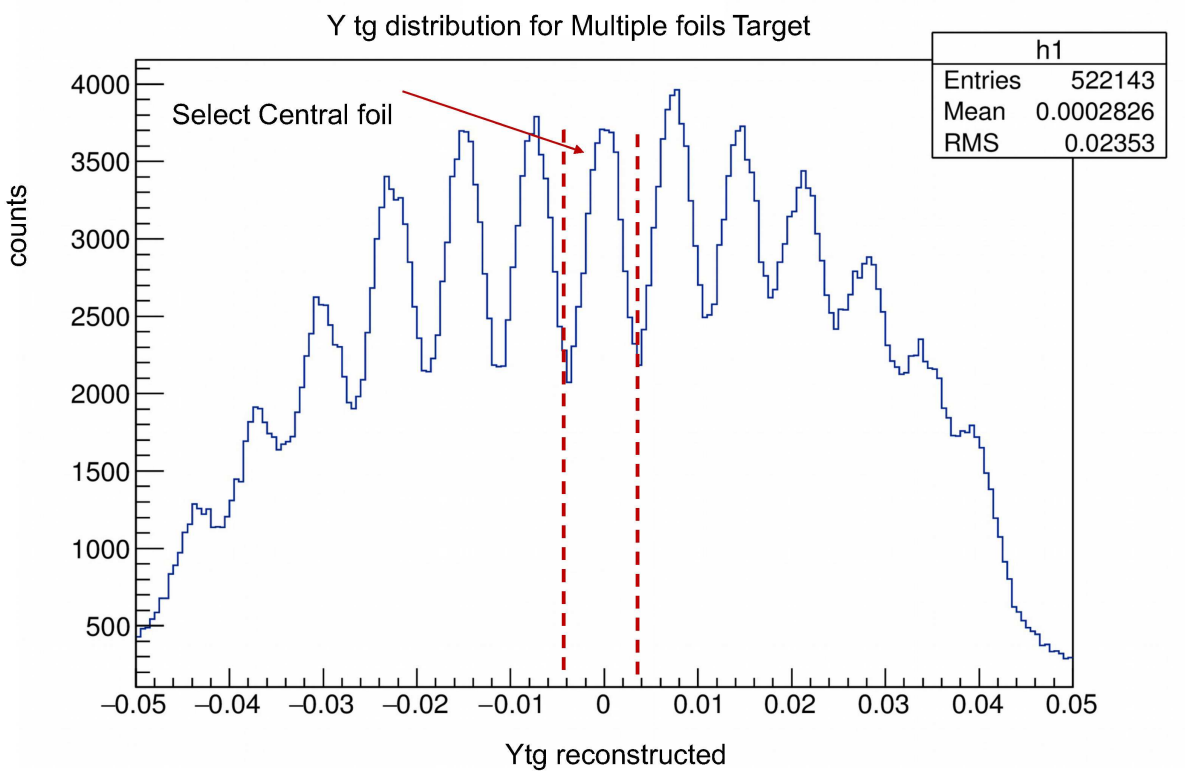

Figure 4.8: The $\mathrm{Y}_{t g}$ distribution of the multiple foils target run. There are 13 foils, each foil is presented by a peak in this plot. The central foil is located at position where $\mathrm{Y}_{t g}$ is close to 0 . The event from the central foil is selected using the $\mathrm{Y}_{t g}$ cut.

the distribution of selected events of the central hole of the central foil as function of the raster current $\mathrm{Y}$ and the out-plane angle. See Figure 4.10. From Figure 4.10, when the raster current increases (ADC channel gets bigger) the out-plane angle goes more negative. This indicates that the beam position moves down in the negative direction. The beam $\mathrm{Y}$ is negative proportional to the raster current $\mathrm{Y}$ or $K_{y}=-1$.

Note that, the raster calibration depends on the beam energy and the beam po- 


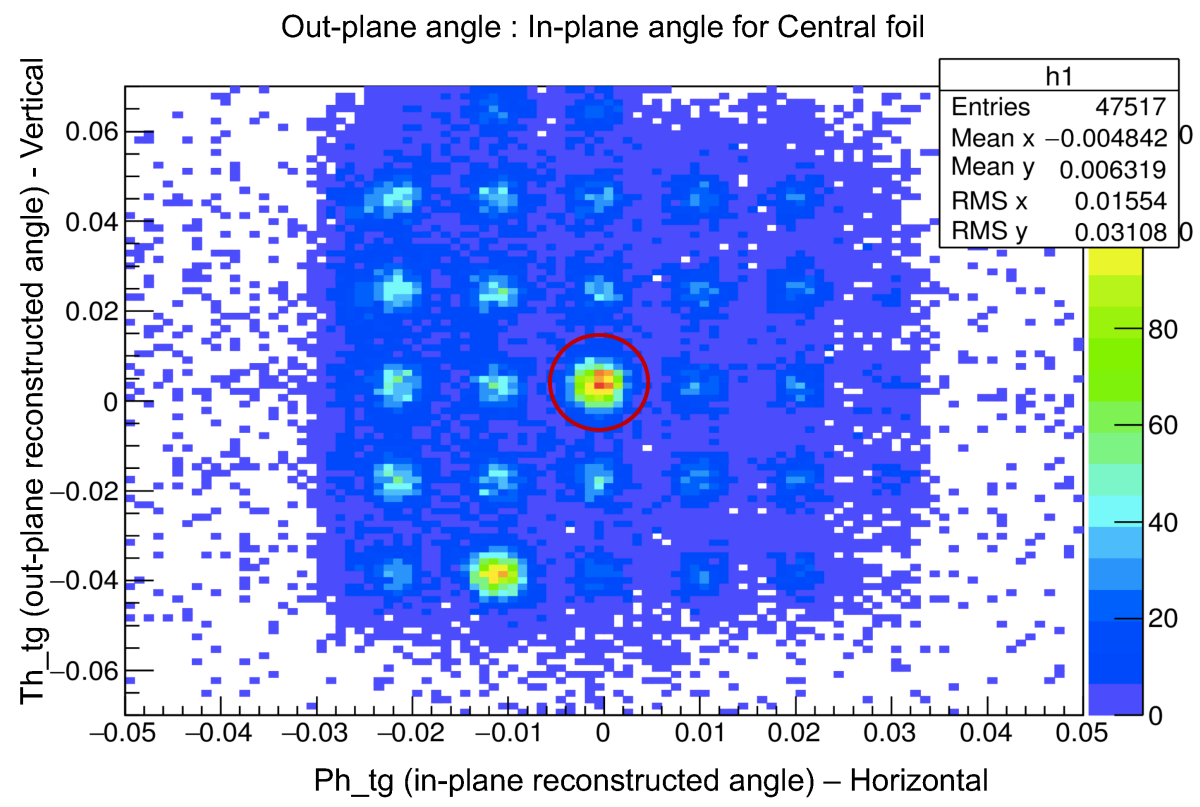

Figure 4.9: The plot show the sieve pattern of the selected event from the central foil (See Figure 4.8). The events from the sieve's central hole are selected using the cuts on in-plane-angle and out-of-plane angle, indicated as red circle in the plot.

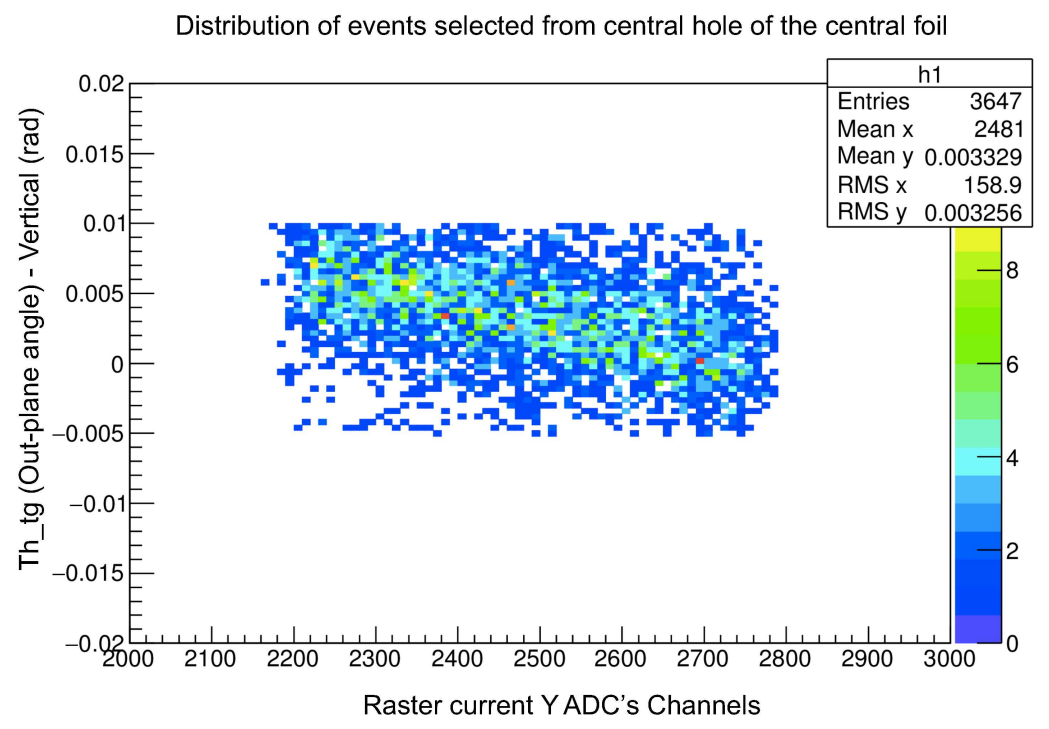

Figure 4.10: Determining of the sign of $K_{y}$ for raster calibration using the distribution of events from the central hole corresponding to the central foils of the optics data.

sition. It means that different beam energies or different beam positions we need to have a different calibration. The raster coefficients can only reflect the beam position which was used in calibration and does not take into account beam movement. The 
easiest solution, and that taken, is to keep beam position fixed.

\subsection{Detector calibration}

\subsubsection{VDC Calibration}

The VDC configuration and their working principle was discussed in Section 3.6.1. The drift times of all the wires in each wire plane are measured using the Time-toDigital Converter (TDC) [59]. The TDCs were operated in the common-stop mode which means that larger TDC channel correspond to shorter drift time and vice versa. Figure 4.11 (left panel) shows the drift time spectrum of the VDC wire plane. Different regions in the drift time spectrum can be understood based on the position of the trajectory in the drift cell, see Figure 4.11 (right panel) 65
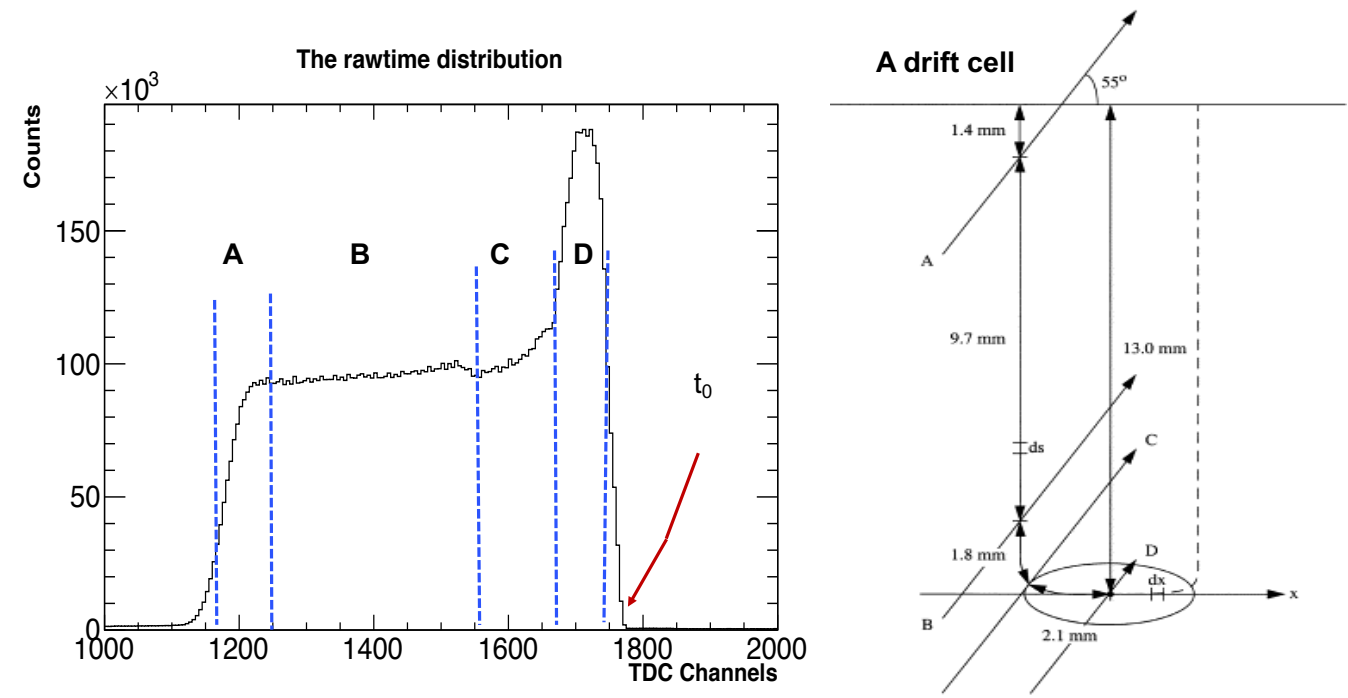

Figure 4.11: Left plot: the VDCs raw-time distribution as a function of TDC channels. Right plot: A drift cell for a wire with 4 different trajectories crossing the cell.

- Region A: This region corresponds to the particles that have a large trajectory angle and small intersection path with the drift cell. These particles are further away from the sense wise and have less ionization which give them a smaller 
probability to be detected.

- Region B: In this region, the particles trajectories cross an area where the field lines are parallel and the drift velocity almost a constant. The drift time is flat for this region.

- Region C: Here the particle trajectory gets close to the wire where the field lines change from parallel to quasi-radial. The detection probability increases in this region and it corresponds to a transition in the drift time spectrum.

- Region D: The particle trajectories cross the quasi-radial field line region where track density is a maximum and the drift velocity is increasing rapidly. The probability of detecting a particle in this region is maximum and it corresponds to the peak in the drift time spectrum.
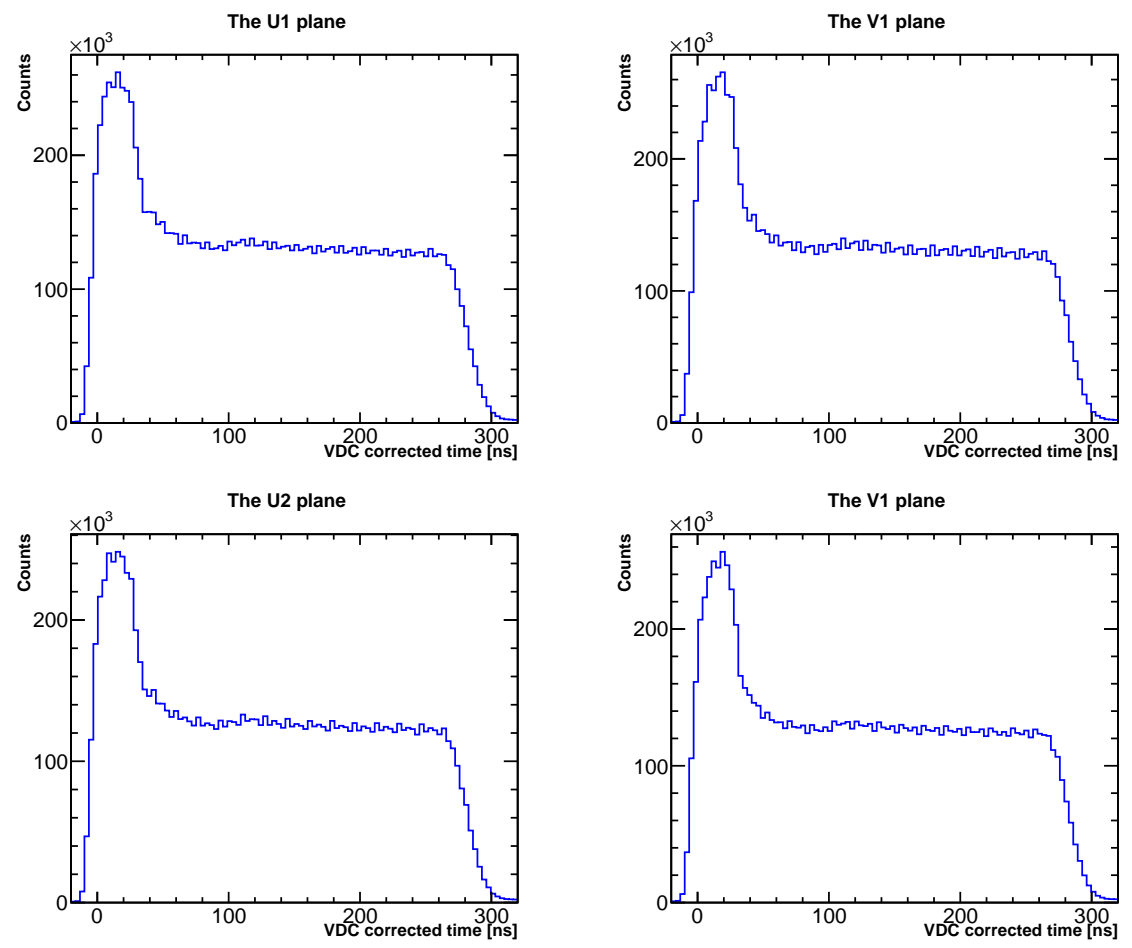

Figure 4.12: The VDC corrected time distribution after the VDC calibration for different wire planes. 
The main goal of VDC calibration is to determine $t_{0}$, the time offset for each wire, in order to compare the drift times of all the wires in a plane. This time offset $t_{0}$ comes from the variations in cable lengths and signal processing times. The first step of the VDC calibration is to find the rising edge of the drift time spectrum and then calculate the derivatives numerically in this region. Next the maximum slope is identified and extrapolated to the drift time axis and the intercept used to define $t_{0}$. Figure 4.12 shows the VDC corrected time after the VDC calibration.

\subsubsection{Cerenkov's ADC calibration}

As mentioned in Section 3.6.3, the Cherenkov detector is responsible for discriminating electrons from pions. The Chereknov sum signal is used for this in the analysis. However the Gas Cherenkov includes 10 individual PMTs and they have different gain factors. Before making a sum from 10 individual signals, a calibration is needed to make sure every PMTs have the same response to the same signal. The goal of this calibration is to align the single photon electron peak (SPE) at ADC channel 100 for each PMT. The SPE indicates the response of the PMTs when only one photoelectron is emitted and we can use it as a unit for measurement of total number of photo-electrons. The gain factors is calculated using

$$
\mathrm{C}=\frac{100}{\mathrm{SPE}_{\text {obser }}-\mathrm{PED}}
$$

where $\mathrm{SPE}_{\text {obser }}$ is the observed SPE channel before calibration and PED is the pedestal channel. In this experiment the Gas Cherenkov was included in the main trigger with a threshold level so we can not see the SPE when using the main trigger. Any other trigger which does not include the Gas Cherenkov can be used to study the SPE. The previous calibration result was used to check the alignment of SPE. In Figure 4.15, it can be seen that only 7 out of 10 PMTs were aligned in initial calibration. 
A new calibration was done. The first step is to determine the PED. The pedestal subtracted signal is used to find the SPE and calculate the new coefficients. Figure 4.13 shows the ADC channel distribution of PMT1 before and after calibration.

LHRS cer_PMT1 before calibration

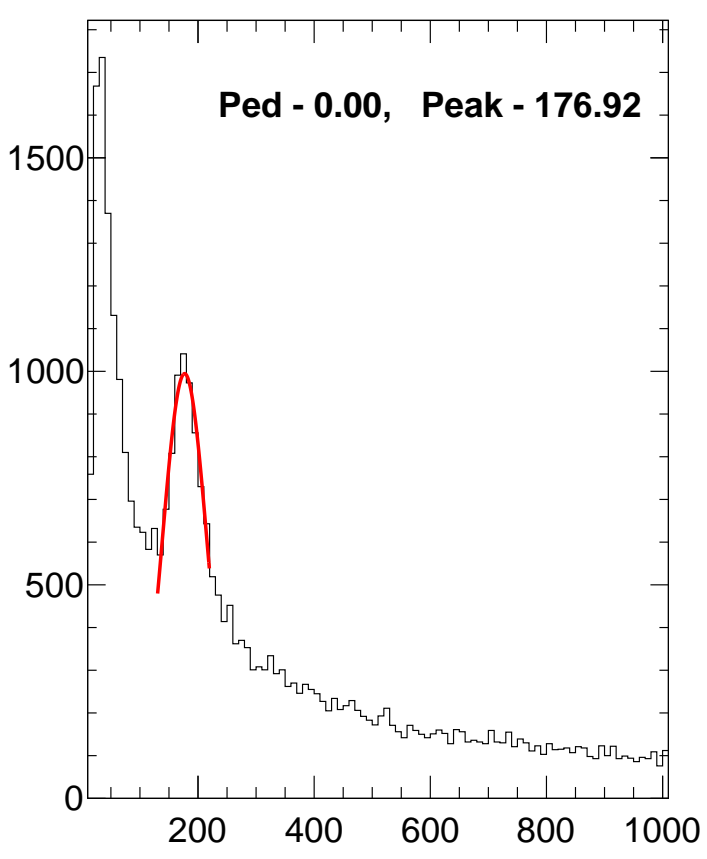

LHRS cer_PMT1 after Calibration

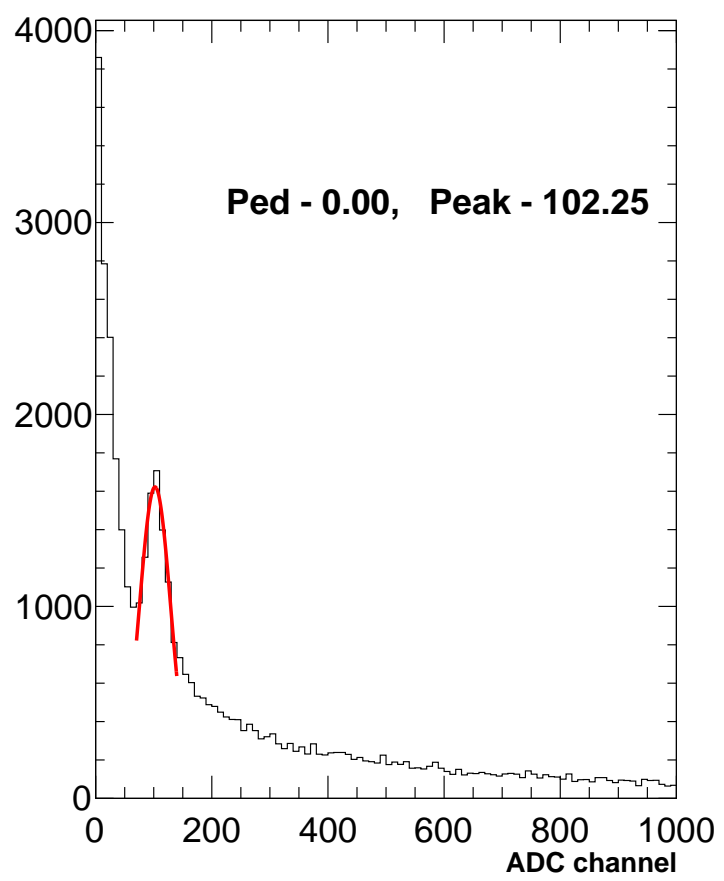

Figure 4.13: The SPE location before and after calibration for PMT1.

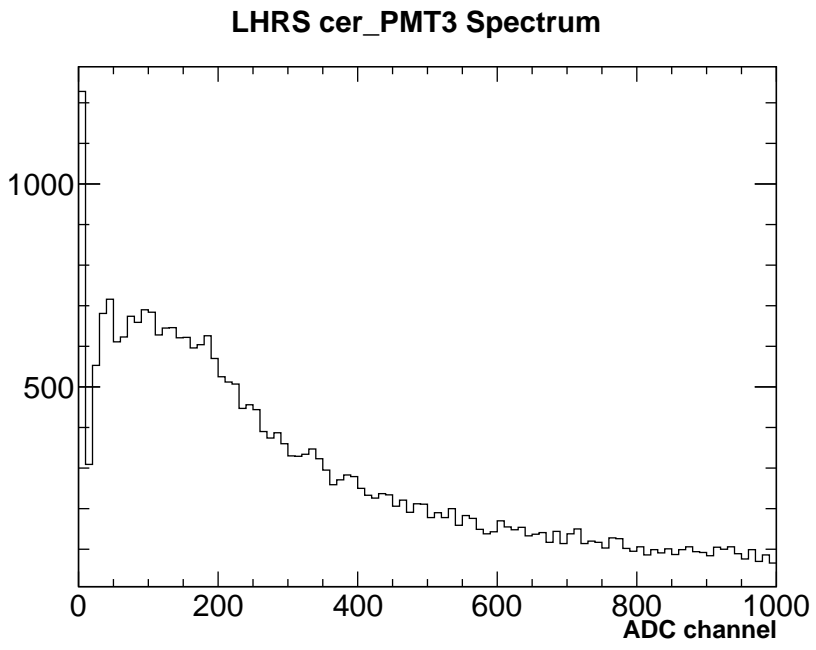

Figure 4.14: The PMT3's ADC spectrum. This is the noisy PMT. No SPE peak is seen and the pedestal is not easily determined. 
Applying these new coefficients we can check the alignment again on the same data and it is much improved, as seen in Figure 4.16. The alignment works better after the new calibration. It was found that PMT3 was a bit noisier compared to the other PMTs and it was harder to define the PED or the SPE for this PMT. See Figure 4.14.

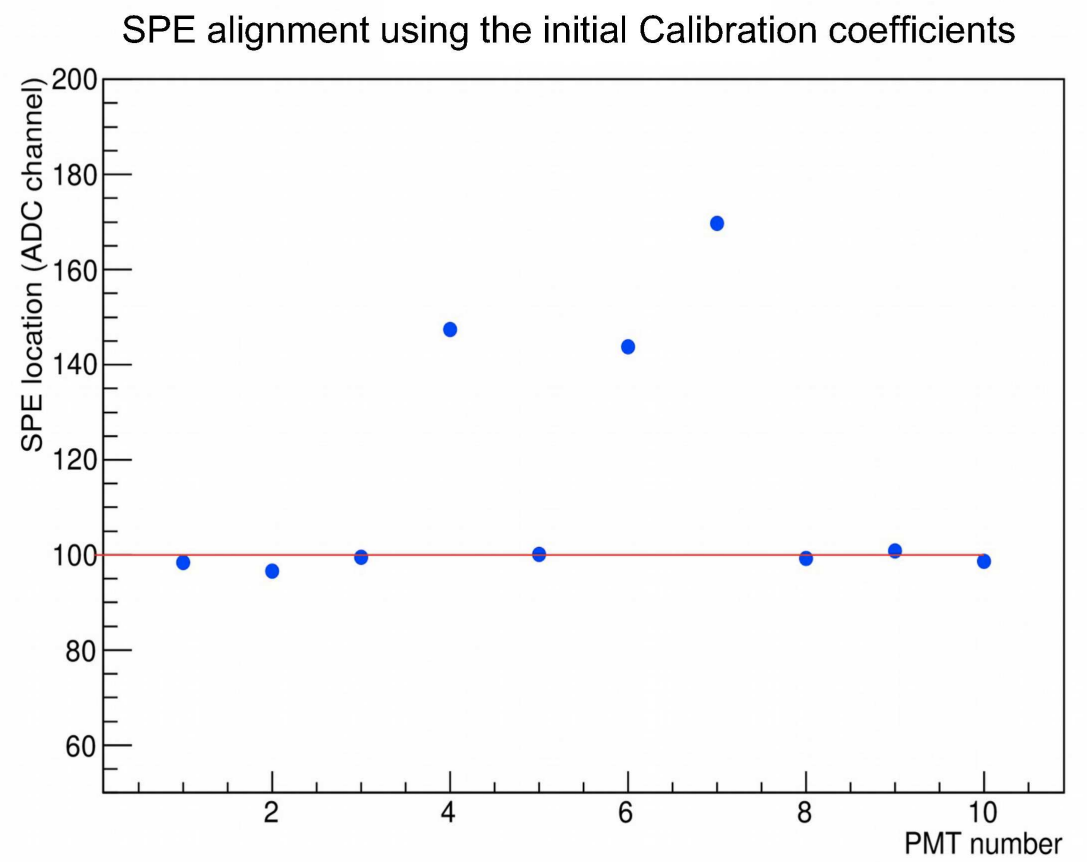

Figure 4.15: The SPE alignment using the initial calibration coefficient.

\subsubsection{Calorimeter Calibration}

The configuration and working principles of the calorimeter were mentioned in Section 3.6.4. The calorimeter provides a additional electron/pion discrimination working in combination with the Cherenkov. The calorimeters in both LHRS and RHRS are segmented into many individual lead-glass blocks, each of them is connected to a PMT. When a high energy particle passes through calorimeter it can produce $\gamma, e^{+}, e^{-}$. This process continues through the calorimeter and creates a cascade of secondary particles. The energy of original particle is converted to the light and 


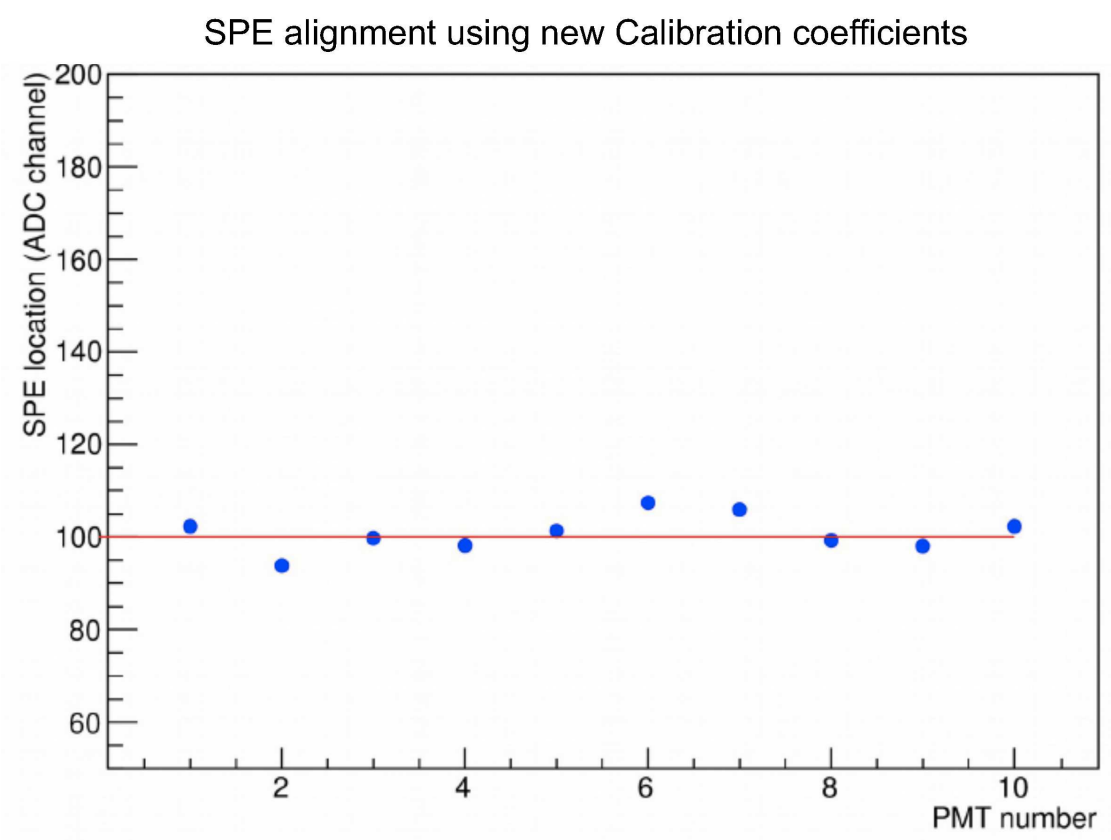

Figure 4.16: The SPE alignment with new calibration.

recorded by the PMTs. Normally, a cascade can fire several lead-glass blocks at the same time which defines a shower cluster. In order to get the deposited energy $(E)$ of particle, the recorded ADC signal from the PMTs needs to be converted to energy and summed over from all adjacent blocks, as in Equation 4.6 .

$$
E=\sum_{i}^{n} E_{i}=\sum_{i}^{n} C_{i} \times A_{i}
$$

where $E_{i}$ is the energy in block $i$ and $n$ is number of block in a cluster. $A_{i}$ is the recorded $\mathrm{ADC}$ value from block $i$ and the $C_{i}$ is the coefficient to convert the ADC channel to energy.

The main goal of the calorimeter calibration is to determine the $\mathrm{C}_{i}$ 's such that the total deposited energy is equal to the momentum of the electron or $E / p \sim 1$. The calibration is based on $\chi^{2}$ minimization where $\chi^{2}$ is defined as:

$$
\chi^{2}=\sum_{i}^{N}\left(\sum_{j}^{n_{1}} C_{j} A_{j}^{i}+\sum_{k}^{n_{2}} C_{k} A_{k}^{i}-P^{i}\right)^{2},
$$


with $P^{i}$ the momentum of event $i, n_{1}$ and $n_{2}$ are number of the block in a cluster in prl1 (pre-shower), prl2 (shower) for the LHRS (RHRS), respectively. $N$ is total number of events selected for the calibration. There is a difference in the LHRS and RHRS calorimeter calibration. As the RHRS total energy is deposited while in LHRS it is not. Details can be found in Refs. 54, 69.

Figure 4.17 shows the performance of the calorimeter after calibration at different kinematics. The left plot is for $P_{0}=2.505 \mathrm{GeV}, \theta_{0}=25^{\circ}$ and the right plot is for $P_{0}=3.035 \mathrm{GeV}, \theta_{0}=23^{\circ}$. The peak is nicely located around 1 and the width of the peak implies a resolution around $5 \%$ in both cases.

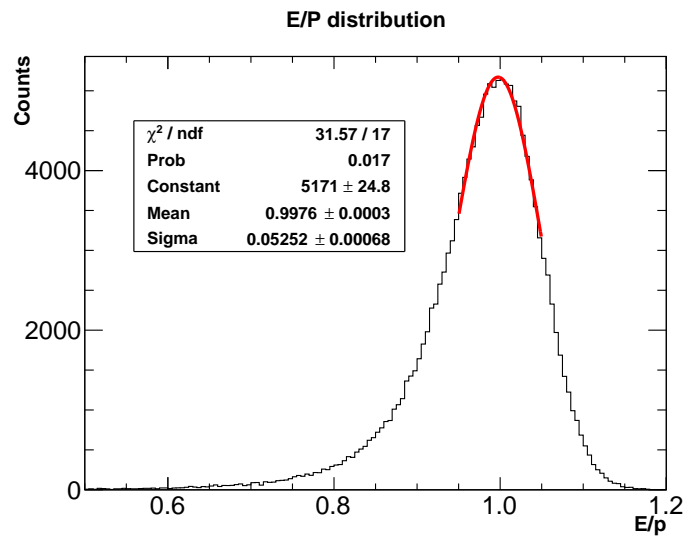

(a) The E/p for kin5.0

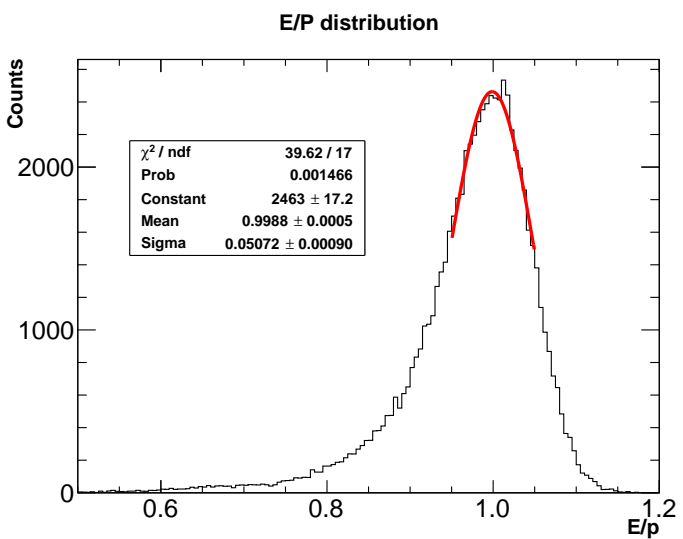

(b) The E/p for kin4.2

Figure 4.17: $E / P$ distribution after calibration. The left plot is for kin5.0, central momentum $P_{0}=2.505 \mathrm{GeV}$ and $\theta_{0}=25^{\circ}$. The right plot is for kin4.2, central momentum $P_{0}=3.035 \mathrm{GeV}$ and $\theta_{0}=25^{\circ}$. Note that the distribution peaks at $E / p \sim 1$.

\subsection{Optics Calibration}

This section will give a general introduction to the working principles of the optics and optics calibration procedure $[70 \sqrt{72}]$. The goal of this calibration is to determine the optics matrix in order to get the reconstructed target variables $y_{t g}, \theta_{t g}, \phi_{t g}$ and $\delta$ for each event. The idea of this reconstruction problem is that after the electron 
beam scatters in the target, a scattered electron comes out and goes through the magnetic fields of the QQDQ magnet configuration of the Hall A HRS before they are detected in the hut. The optics matrix takes the detected information and returns the reconstructed information including the position and direction of each event at target. The optics matrix is a mathematical expression of the magnetic field of HRSs and we use a polynomial function in this case. We use special data to do the optics calibration. For example, data from the multiple foils target and the sieve slit are used for the position and angle calibration, respectively. We also need a detailed survey for target position as well as spectrometer offsets to calculate the expected value. The calibration is based on a $\chi^{2}$ optimization to determine the coefficients for the polynomial function which form the optics matrix. An introduction to the HRS coordinate systems is provided in the following section in order to understand the calculation of expected quantities.

\subsubsection{HRS coordinate systems}

There are five different right handed coordinate systems used in the HRS analysis. Only a short introduction is presented here, a detailed description for each of coordinate system can be found in Ref. [70].

- Hall Coordinate system (HCS): The origin of HCS is the Hall A center, which is defined as intersection of the electron beam and the vertical axis of the target. The positive $z$ is along the beam direction, positive $y$ is vertical up and positive $x$ pointing to the left of the beam direction as seen in Figure 4.18

- Target coordinate system (TCS): The $z_{t g}$ is defined as central ray of the spectrometer which is perpendicular to the sieve slit surface and goes through the sieve slit central hole. The positive $z_{t g}$ points away from the target. The $y_{t g}$ axis points away from beam direction. The $x_{t g}$ axis points down as can be 


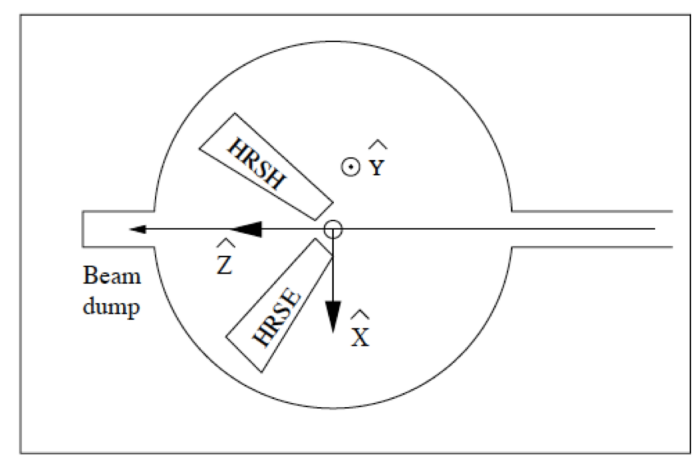

Figure 4.18: Hall coordinate system (HCS) (top view).

seen in Figure 4.19, In the ideal situation where the beam, spectrometer and sieve slit have no offset, the origin of the TCS is the same as hall center.

- Detector coordinate system (DCS): The intersection of wire 184 of the VDC1 U1 plane and the perpendicular projection of wire 184 in the VDC V1 plane onto the VDC U1 plane defines the origin of DCS. The $z$ axis is perpendicular to VDC1 U1 plane pointing vertically up, $x$ is along a symmetry axis of the lower VDC pointing away from the hall center See Figure 4.20.

- Transport coordinate system (TRCS): The TRCS is generated by rotating the DCS clockwise around its $y$ axis by $45^{\circ}$, so that the $z$ axis coincides with the central ray of the spectrometer in the ideal case See Figure 4.21. The TRCS is used to transport the DCS to the focal plane coordinate system which is used in the optimization, described in the later section. The TRCS coordinates of each event can be expressed in term of DCS coordinates by the following equation 


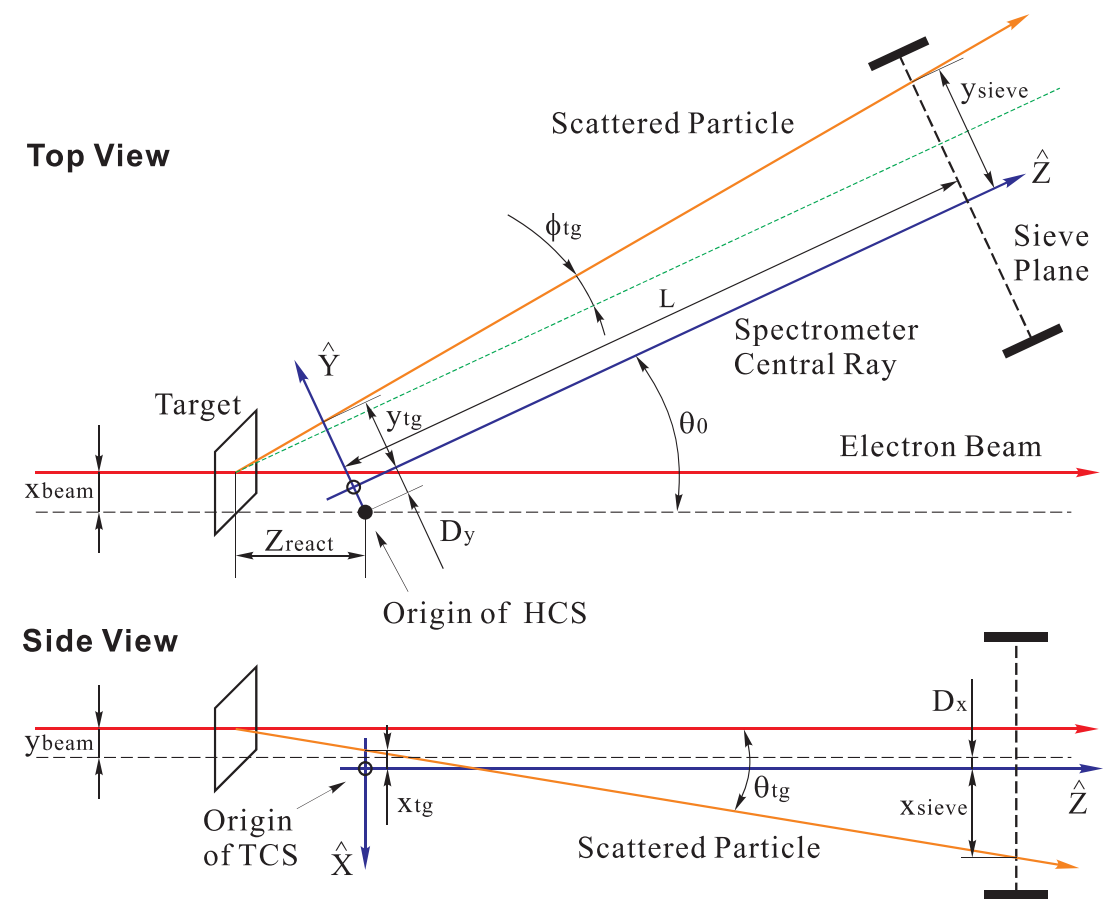

Figure 4.19: Target coordinate system for top view and side view.
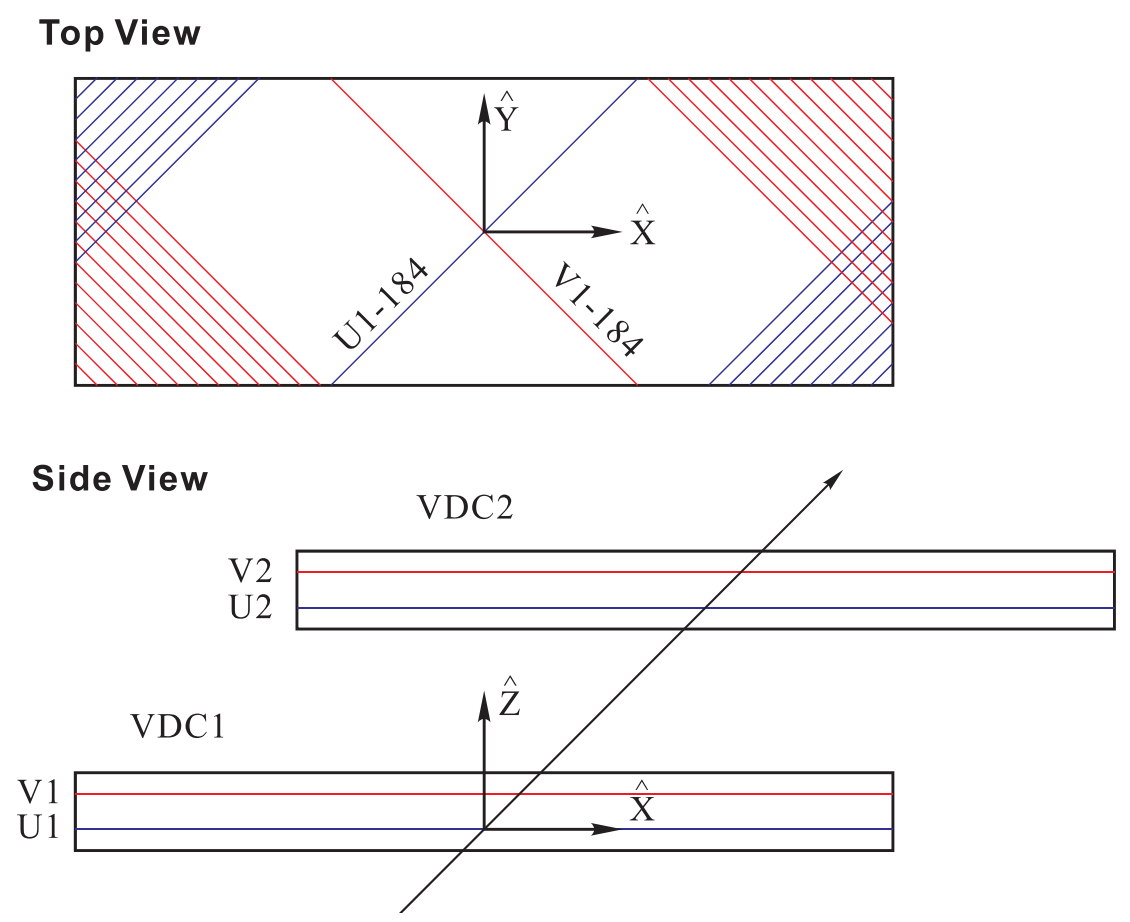

Figure 4.20: Detector coordinate system, top and side view. 
4.8 .

$$
\begin{aligned}
\theta_{\text {tran }} & =\frac{\theta_{\text {det }}+\tan \rho_{0}}{1-\theta_{\text {det }} \tan \rho_{0}} \\
\phi_{\text {tran }} & =\frac{\phi_{\text {det }}}{\cos \rho_{0}-\theta_{\text {det }} \sin \rho_{0}} \\
x_{\text {trans }} & =x_{\text {det }} \cos \rho_{0}\left(1+\theta_{\text {tran }} \tan \rho_{0}\right) \\
y_{\text {tran }} & =y_{\text {det }}+\sin \rho_{0} \phi_{\text {tran }} x_{\text {det }}
\end{aligned}
$$

and $\rho_{0}=-45^{\circ}$ is the rotation angle.

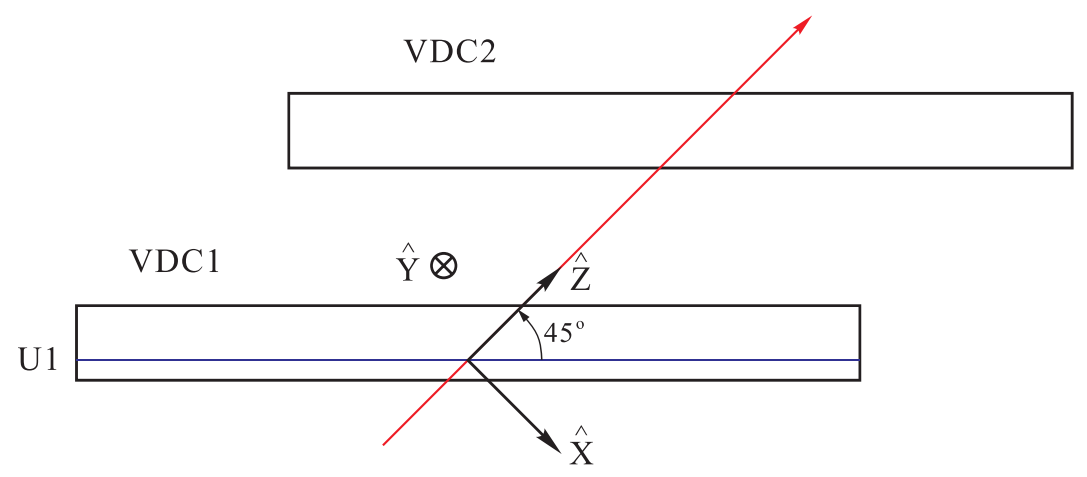

Figure 4.21: Transport coordinate system (side view).

- Focal plane coordinate system (FCS): The FCS is the coordinate system, generated by rotating the DCS clockwise around its $y$ axis by the angle $\rho$ where $\rho$ is the angle between the local central ray and the $z$ axis of the DCS, see Figure 4.22. This coordinate is chosen for the HRS analysis and can be calculated from 
the TRCS coordinates by following equation:

$$
\begin{aligned}
x_{f p} & =x_{\text {tran }} \\
\tan \rho & =\sum t_{i 000} x_{f p}^{i} \\
y_{f p} & =y_{\text {tran }}-\sum y_{i 000} x_{f p}^{i} \\
\theta_{f p} & =\frac{\theta_{\text {det }}+\tan \rho}{1-\theta_{d e t} \tan \rho} \\
\phi_{f p} & =\frac{\phi_{\text {det }}-\sum p_{i 000} x_{f p}^{i}}{\cos \rho-\theta_{\text {det }} \sin \rho},
\end{aligned}
$$

where $t_{i 000}, y_{i 000}, p_{i 000}$ are the terms in the optics matrix which were calibrated based on the offset of VDC package.

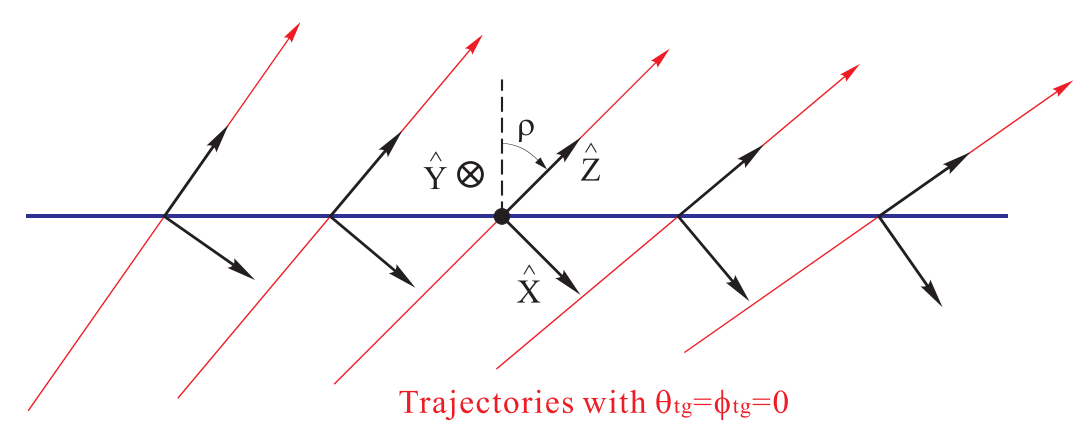

Figure 4.22: Focal plane coordinate system (side view).

\subsubsection{Optimization procedure}

\section{Expected target variables}

Using the survey information and the geometry of the spectrometer the expected value for each target variable can be determined using the Equation 4.10. This is the 
first step forward calculating the $\chi^{2}$ for optimization.

$$
\begin{aligned}
\theta_{t g} & =\frac{x_{\text {sieve }}+D_{x}+y_{\text {beam }}}{L-Z_{\text {react }} \cos \left(\theta_{0}\right)-x_{\text {beam }} \sin \left(\theta_{0}\right)} \\
\phi_{t g} & =\frac{y_{\text {sieve }}+D_{y}-x_{\text {beam }} \cos \left(\theta_{0}\right)+Z_{\text {react }} \sin \left(\theta_{0}\right)}{L-Z_{\text {react }} \cos \left(\theta_{0}\right)-x_{\text {beam }}} \\
x_{t g} & =x_{\text {sieve }}-L \theta_{t g} \\
y_{t g} & =y_{\text {sieve }}-L \phi_{t g}
\end{aligned}
$$

$L, D_{x}, D_{y}$ come directly from spectrometer survey. For more details see Figure 4.19 These target variables are used to calculate the scattering angle and the reaction points along the beam $\left(\mathrm{Z}_{\text {react }}\right)$ using:

$$
\begin{aligned}
\theta_{\text {scat }} & =\arccos \left(\frac{\cos \theta_{0}-\phi_{t g} \sin \theta_{0}}{\sqrt{1+\theta_{t g}^{2}+\phi_{t g}^{2}}}\right) \\
z_{\text {react }} & =\frac{-\left(y_{t g}+D_{y}\right)+x_{\text {beam }}\left(\cos \theta_{0}-\phi_{t g} \sin \theta_{0}\right)}{\cos \theta_{0} \phi_{t g}+\sin \theta_{0}}
\end{aligned}
$$

where $\theta_{0}$ is the central angle of the spectrometer. The $x_{\text {sieve }}$ and $y_{\text {sieve }}$ come from the sieve survey.

\section{Reconstructed target variables from data}

In the DCS coordinates, for each event, two angle coordinates $\theta_{\text {det }}$ and $\phi_{\text {det }}$ and two spatial coordinates $x_{d e t}$ and $y_{\text {det }}$ are directly measured with the VDCs. These variables are corrected for any detector offsets to provide the focal plane variables which are used to calculate the reconstructed variables at the target. The transformation between focal plane variables to the target variables can be written as: 


$$
\left(\begin{array}{l}
\delta \\
\theta \\
y \\
\phi
\end{array}\right)_{t g}=\left(\begin{array}{cccc}
<\delta \mid x> & <\delta \mid \theta> & 0 & 0 \\
<\theta \mid x> & <\delta \mid \theta> & 0 & 0 \\
0 & 0 & <y \mid y> & <y \mid \phi> \\
0 & 0 & <\phi \mid y> & <\phi \mid \phi>
\end{array}\right)\left(\begin{array}{l}
x \\
\theta \\
y \\
\phi
\end{array}\right)_{f p}
$$

The relative momentum $\delta$ is defined as:

$$
\delta=\frac{P-P_{0}}{P_{0}}
$$

where $P$ is the measured momentum of the particle and $P_{0}$ is the central momentum setting of the spectrometer. In the optics calibration, to reduce the number of unknown variables at target, the $x_{t g}$ is effectively set at zero. Other target variables $\delta, \theta_{t g}, \phi_{t g}$ and $y_{t g}$ can be calculated using the initial optics matrix following by Expression 4.12 .

$$
\begin{aligned}
y_{t g} & =\sum_{j, k, l} Y_{j k l} \theta_{f p}^{j} y_{f p}^{k} \phi_{f p}^{l} \\
\theta_{t g} & =\sum_{j, k, l} T_{j k l} \theta_{f p}^{j} y_{f p}^{k} \phi_{f p}^{l} \\
\phi_{t g} & =\sum_{j, k, l} P_{j k l} \theta_{f p}^{j} y_{f p}^{k} \phi_{f p}^{l} \\
\delta & =\sum_{j, k, l} D_{j k l} \theta_{f p}^{j} y_{f p}^{k} \phi_{f p}^{l},
\end{aligned}
$$

where the tensors $Y_{j k l}, T_{j k l}, D_{j k l}$ are polynomials in $x_{f p}$, for example:

$$
Y_{j k l}=\sum_{i} C^{Y_{j k l}} x_{f p}^{i} .
$$




\section{$\chi^{2}$ minimization}

For each event the $\chi^{2}$ is defined as the variance of reconstructed data from their expected values.

$$
\chi^{2}=\sum_{N}\left(\sum_{j, k, l} X_{j k l} \theta_{f p}^{j} y_{f p}^{k} \phi_{f p}^{l}-X^{0}\right)^{2}
$$

where $\mathrm{N}$ is total number of measured events for the calibration. The $X_{j k l}$ can be any of the tensors $Y_{j k l}, T_{j k l}, P_{j k l}, D_{j k l}$ and the $X^{0}$ can be the expectation value for any variable $y_{t g}, \theta_{t g}, \phi_{t g}, \delta$. A optimization package, TMINUIT, minimizes $\chi^{2}$ to determine the optics matrix elements for all target variables.

\subsubsection{Reconstruction after optimization}

E08014 did not have enough optics data nor sufficient survey data so the optics calibration did not perform well. The initial optics matrix for this analysis had a reconstruction issue for the $\theta_{t g}$ variables, and detailed information will be provided in Section 4.6. In principle the optics is sensitive to the magnet tune of the spectrometer, i.e, the ratio of magnetic field in Quadrupoles (Q) to the magnetic field in Dipole (D). If the magnets follow the same tune, the same optics matrix can be used in the nominal range of momentum and angular acceptance. The $\mathrm{G}_{M}^{p}$ experiment (E12-07-108) ran in Spring 2016 with high precision optics using the same magnetic tune, so their data was used in this analysis. The following is the reconstruction check using the $\mathrm{G}_{M}^{p}$ optics matrix elements on both position, the angle and momentum [73, 74].

- Position reconstruction $\mathrm{Z}_{\text {vertex }}$ : The multiple foil target which covers the full length of long target was used to take data for the position calibration See Figure 4.23 .

- Angle reconstruction $\theta_{t g}, \phi_{t g}$ : The sieve slit data taken with multiple foils target was used for the angle calibration See Figure 4.24 . 

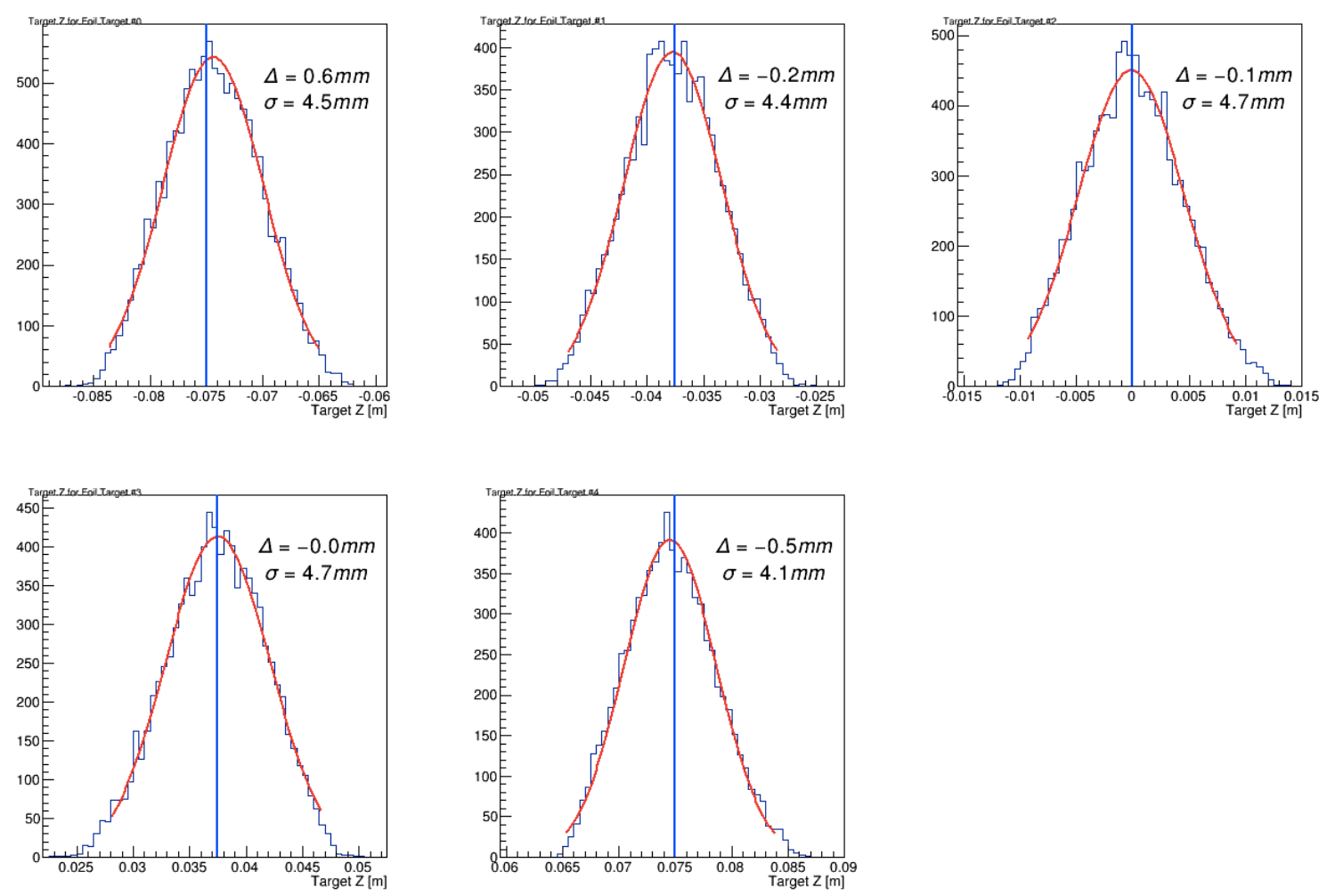

Figure 4.23: $\mathrm{Z}_{\text {vertex }}$ after the optimization in $\mathrm{G}_{M}^{p}$ optics using Spring 2016 optics data. The blue lines are the expected values. The black curves are the event distribution for different target foils.

- Relative momentum reconstruction: See Figure 4.25

\subsubsection{Performance of $\mathrm{G}_{M}^{p}$ optics on E08014 data}

Applying the $\mathrm{G}_{M}^{p}$ optics on the E08014 optics data as check of the performance of the optics matrix.

\section{Multiple foils target data}

Using the multiple foils target data, we can check the position reconstruction performance See Figures 4.26 and 4.27 . 
Sieve Plane Proj. (tg_Xvs tg_Y) for Data set \#0

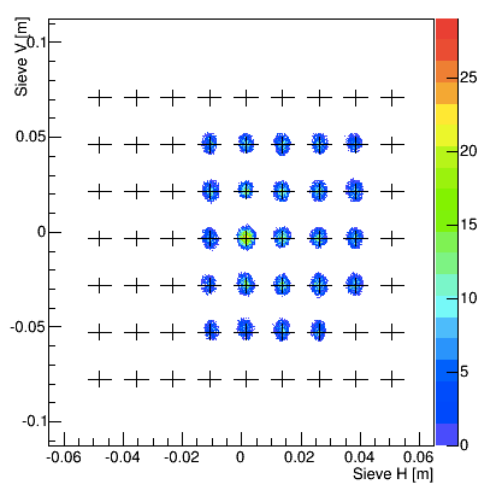

Sieve Plane Proj. (tg_X vs tg_Y) for Data set \#3

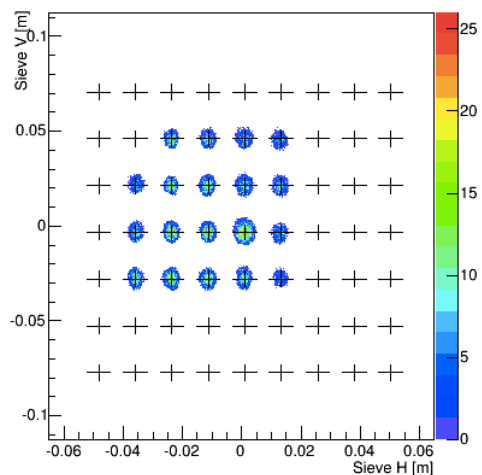

Sieve Plane Proj. (tg_X vs tg_Y) for Data set \#1

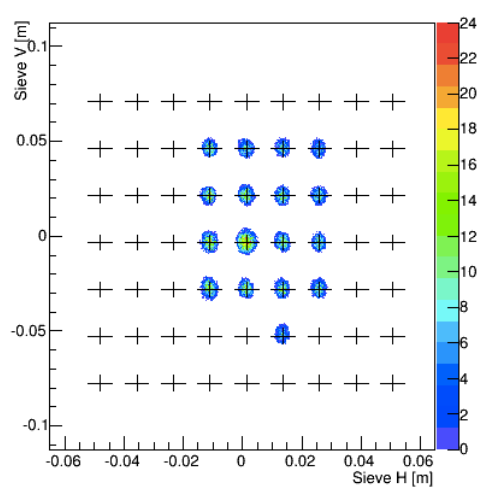

Sieve Plane Proj. (tg_X vs tg_Y) for Data set \#4

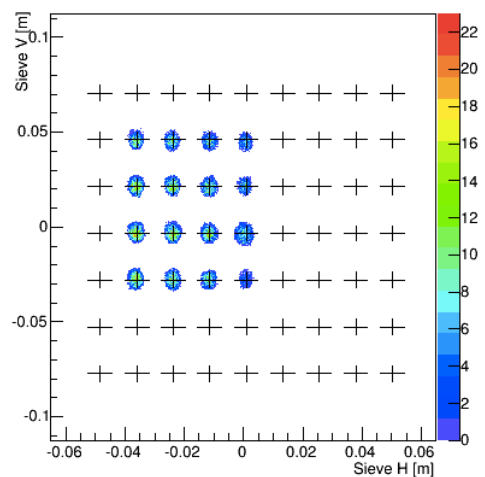

Sieve Plane Proj. (tg_ X vs $\operatorname{tg} Y$ Y ) for Data set \#2

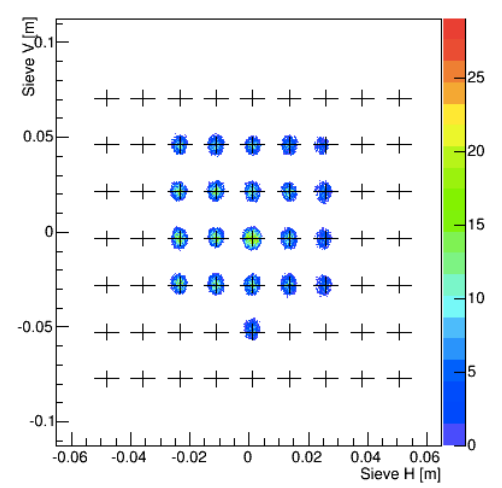

Figure 4.24: Reconstructed angle after the optimization in $\mathrm{G}_{m}^{P}$ optics using Spring 2016 optics data. The cross points present the expected position of each sieve hole. The event distribution after optimization for each sieve hole shows a good reconstruction.

\section{Multiple foils targets with sieve on data}

Using the sieve on data on the multiple foils target we can check the angle reconstruction performance of the optics See Figure 4.28 .

The optics matrix using the $\mathrm{G}_{M}^{p}$ data performs well for the E08014 data and is used for this analysis. 
Dp_Kin for Delta Scan Kine. -4\%

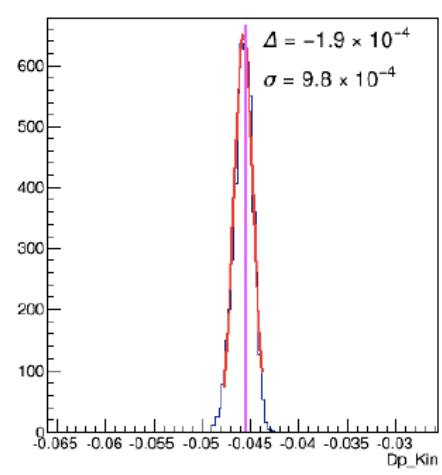

Dp_Kin for Delta Scan Kine. - $2 \%$

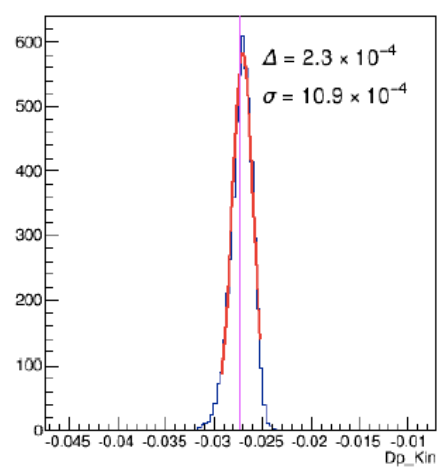

Dp_Kin for Delta Scan Kine. 0\%

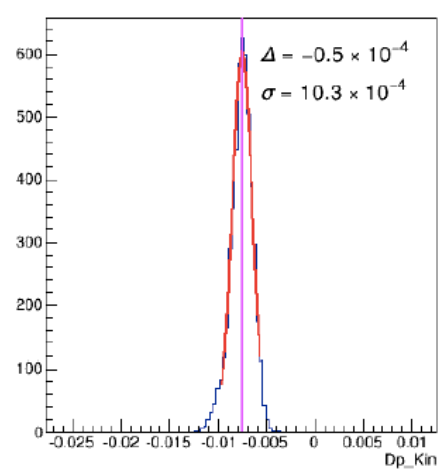

Figure 4.25: Relative momentum reconstruction after the optimization in $G_{m}^{P}$ optics using Spring 2016 optics data. The blue lines are the expected value of the relative momentum and the black curves are the event distribution after the optimization. The red curves are a gaussian fit to the event distribution. The reconstruction has good resolution $\left(\sigma \sim 10^{-3}\right)$

Ytg distribution for multiple foils run

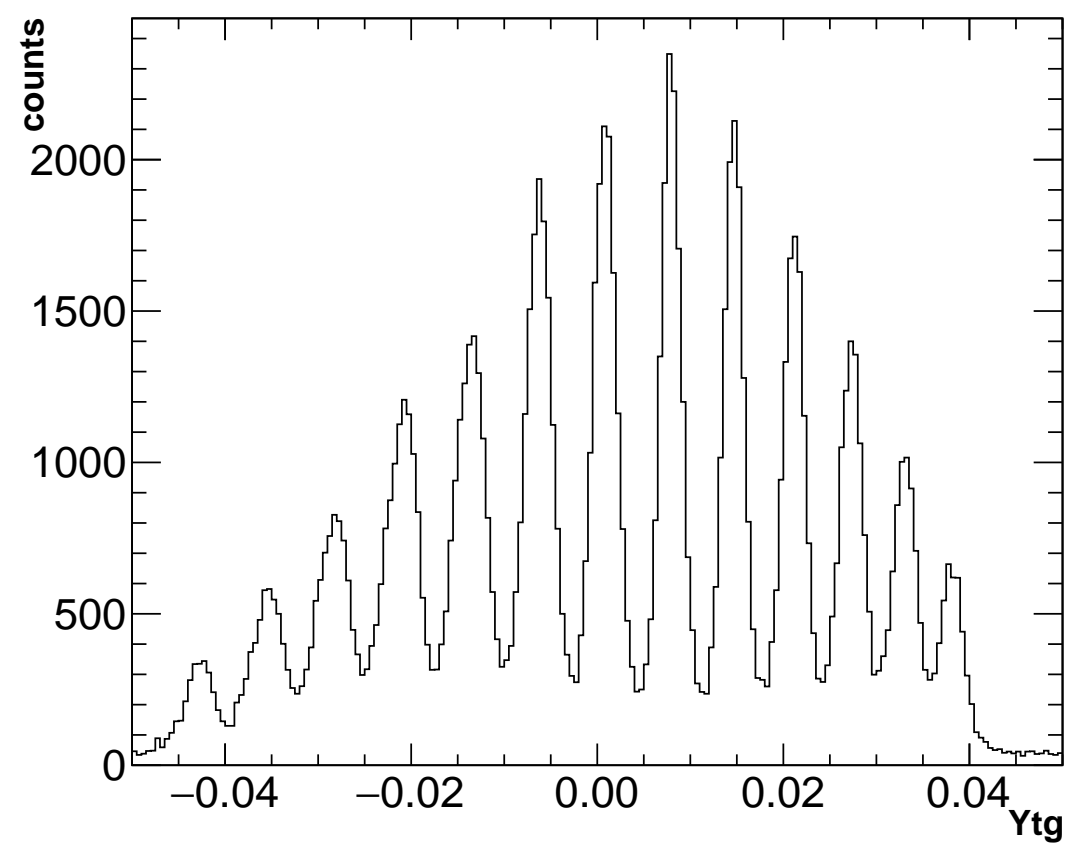

Figure 4.26: Multiple foils target, Y reconstruction 


\section{In plane angle : Ytg distribution}

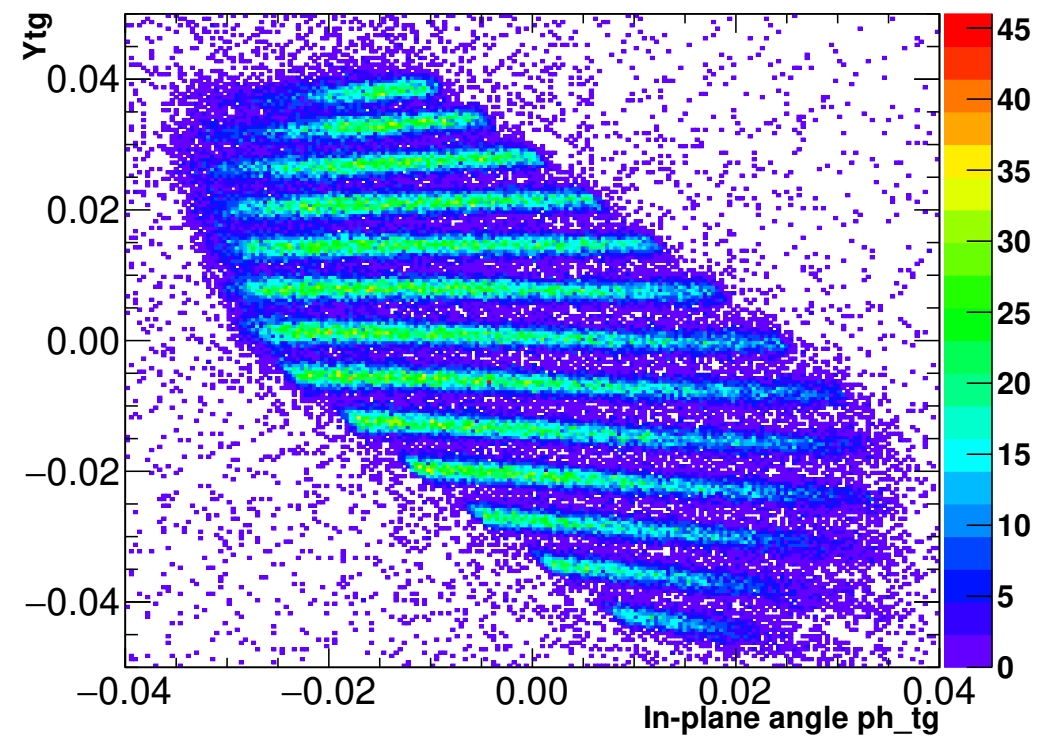

Figure 4.27: Multiple foils target, in plane angle vs Ytg reconstruction. The foils are well separated and the reconstructed positions agree well with the expected position of each foil.

\section{Sieves pattern for central foil}

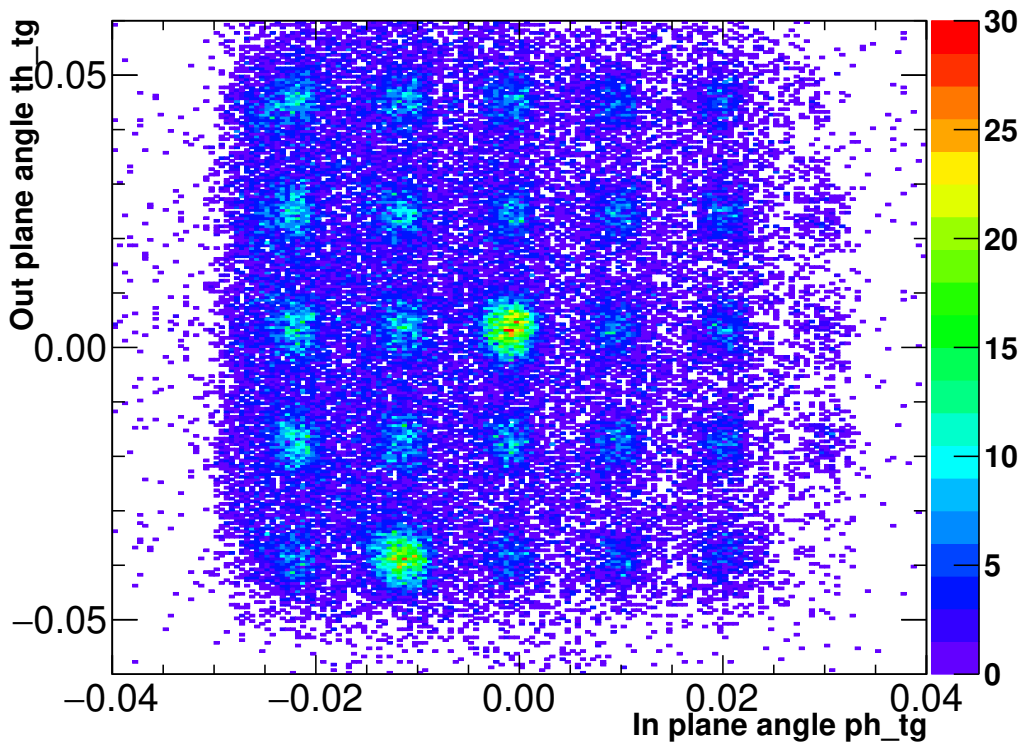

Figure 4.28: Sieves run with multiple foils target. The pattern of the sieve for the central foil. 


\subsection{Efficiency Studies}

\subsubsection{Beam Charge Calculation}

In Hall A BCMs were responsible for measuring the beam current. Scalers were used to record the information from BCM signals and timing signals. The clock sends a pulse signal and get counted in a time scaler. The number of clock count in the scaler increases with time and is proportional to the clock rate See Figure 4.29.

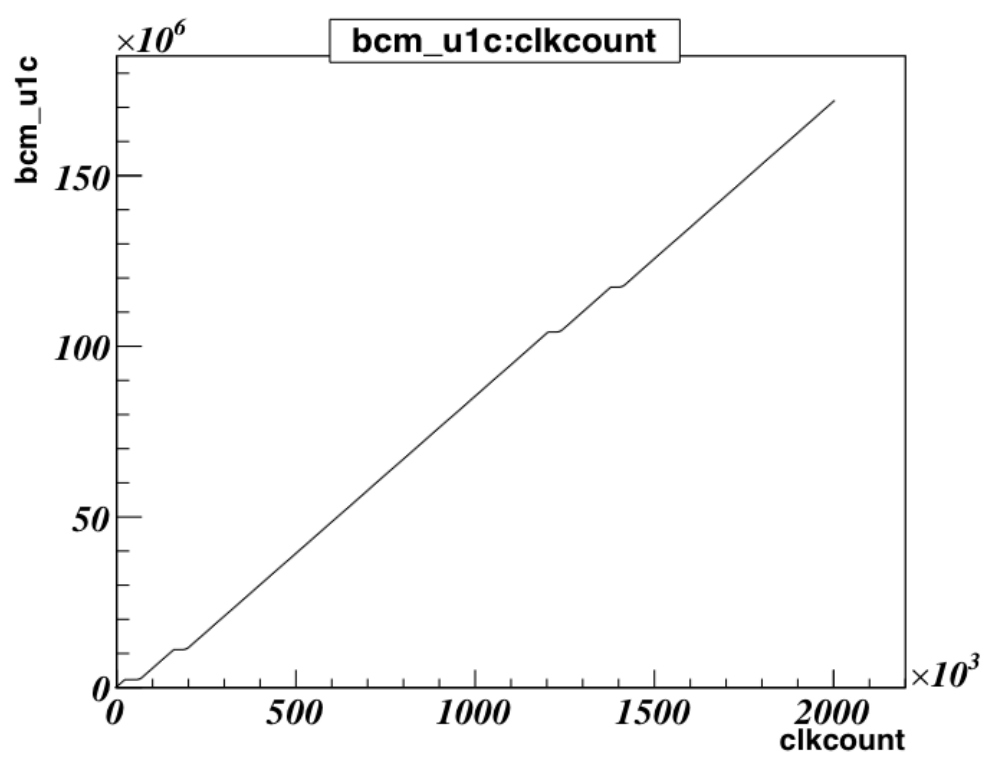

Figure 4.29: The number of BCM counts from bcm-u1 vs number of count from the clock. When beam trips, the bcm count doesn't change.

The clock rate used in this experiment was $1042 \mathrm{~Hz}$, and the number of clock counts was updated every 4 seconds. Using the time scaler we can extract the running time. The BCM scalers record the accumulated counts from BCM signals. The beam current is the pulse signal with frequency, higher current corresponding to higher frequency and can be expressed as:

$$
I=m \times f+b
$$


where $f$ is frequency, $m$ and $b$ are two parameters provided by the BCM calibration [75] ( $m$ is the slope while $b$ is an offset term). The total beam charge is the integration of current over the total time:

$$
Q=\int I d t=\underbrace{\left[m \times \int f d t\right]}_{\text {First term }}+\underbrace{\left[b \times \int d t\right]}_{\text {Second term }} .
$$

The First term is the total count from BCM scalers. The current may change during runtime through changing the frequency but we don't need to worry about the change in $f$, the integral will give total count from BCMs. In an ideal situation when there is no current $f$ is zero and no counts are recorded from BCMs. In the real situation the BCMs have an offset which means that the BCMs still have some counts when there is no beam. The parameter b will take care of the BCM offset. The second term is the total count from the time scaler which provides the run time using:

$$
\int d t=\frac{\mathrm{Clk}_{\text {count }}}{\mathrm{Clk}_{\text {rate }}}
$$

where $\mathrm{Clk}_{\text {count }}$ and $\mathrm{Clk}_{\text {rate }}$ are the number of counts from clock scaler and the clock rate. Beam trips need to be handled. From the plot of BCMs count as function of time, Figure 4.29, BCMs count are constant when there is a beam trip. We only care about total charge when beam is on. To be able to do that we can calculate the accumulated charge and live current between two consecutive scaler events.

$$
\begin{aligned}
\mathrm{BCM}_{\text {count }}^{i} & =\mathrm{BCM}_{i+1}-\mathrm{BCM}_{i} \\
\Delta t^{i} & =\left(\mathrm{Clk}_{i+1}-\mathrm{Clk}_{i}\right) / \mathrm{Clk}_{\text {rate }} \\
I^{i} & =m * \mathrm{BCM}_{\text {count }}^{i} / \Delta t^{i}+b \\
Q^{i} & =I^{i} \cdot \Delta t^{i}
\end{aligned}
$$

We can set the condition for this live current to select events without a beam trip. Two different scalers, Up (U) and Down (D), for both the Left and the Right 
HRS were set up to do the measurement for the same BCMs signal but with different amplification factors $(x 1, x 3, x 10)$, labeled as U1(D1), U3(D3), U10(D10). E08014 used current above $40 \mu \mathrm{A}$ so U10(D10) were unusable. The read-back of the HRS Left scalers was unstable for the entire experiment, so the Right BCM scalers readback was used to determine the charge and current for production data [54,75]. There are two sets of BCM parameters from two calibrations which can be used to estimate the beam charge uncertainty. The difference in the calculated beam charge using two parameter sets the uncertainty at the level of $0.5 \%$.

\subsubsection{Livetime}

The dead-time of concern is the computer dead-time. Events will be skipped if they come while the DAQ is busy processing the current event. The dead-time will be evaluated as the percentage of the trigger events being skipped to the total trigger event over a certain period of time. The dead-time mainly depends on the trigger rate. The dead-time can be reduced by applying a pre-scale factor for each trigger. In this way the DAQ processes at a lower rate so the chance that DAQ misses an event is lower and this means lower dead-time.

Online dead-time is monitored by using the electron dead time monitor module (EDTM) which mixes pulse with fixed frequency send to TDC signal. The total number of pulses in a fixed amount of time is known and total number events detected by DAQ is known as well. So the dead-time is a percentage of events which are not recorded by DAQ. The dead-time can be adjusted by changing the pre-scale factor.

For offline analysis, dead-time is calculated for individual triggers. The average value of the dead-time for the main trigger will be calculated by dividing the total number of events recorded by DAQ (scaled by pre-scale factor) to the total trigger events was sent to DAQ. So the average dead-time (DT) for each trigger can be 
expressed by:

$$
D T_{i}=1-\frac{P S_{i} * N_{i}^{\text {record }}}{N_{i}^{\text {sent }}}
$$

where $\mathrm{DT}_{i}$ is the dead-time for a specific trigger $\mathrm{T}_{i}, \mathrm{PS}_{i}$ is pre-scaler factor, $\mathrm{N}_{i}^{D A Q}$ is the number of events recorded by DAQ and $\mathrm{N}_{i}^{\text {scaler }}$ is the total number of events counted by the scaler for the same trigger. The $\mathrm{N}_{i}^{D A Q}$ can be defined using the trigger type cut while the $\mathrm{N}_{i}^{\text {sent }}$ can be calculated as the total accumulated count of the trigger event using the trigger scaler. The value that is used in data analysis is Livetime (LT) where $L T=1-D T$. The number of good events for each run will be corrected by LT:

$$
N_{E X}=P S_{i} \cdot \frac{N_{i}^{\text {selected }}}{L T_{i}}
$$

where $\mathrm{N}_{i}^{\text {selected }}$ is total number of selected events after selection cuts. After applying the LT correction, the expected total number of events, $N_{E X}$, can be obtained. This will be used in the cross section calculation.

\subsubsection{Particle Identification Efficiency}

The combination of the Gas Cherenkov and the calorimeter are responsible for $e^{-} / \pi$

discrimination. Specifically, the cuts on the Cherenkov sum signal (cer-sum) and the total energy deposited in calorimeter $(E / p)$ are used to separate the $e^{-}$from the $\pi$. These cuts eliminate the most of the $\pi$ contamination but they can also remove some of the good $e^{-}$'s. The goal of the particle identification efficiency study is to find the optimized cuts which minimize the $\pi$ contamination while maximizing the number of good $e^{-}$'s. An understanding of the behavior of different types of particles in the Cherenkov and calorimeter is needed in order to do this study. Good electrons produce a large signal in cer-sum and E/p is centered at 1.0 as seen in Figure 4.30 . The $\pi^{-}$'s do not normally create a Cherenkov signal because of the velocity threshold 

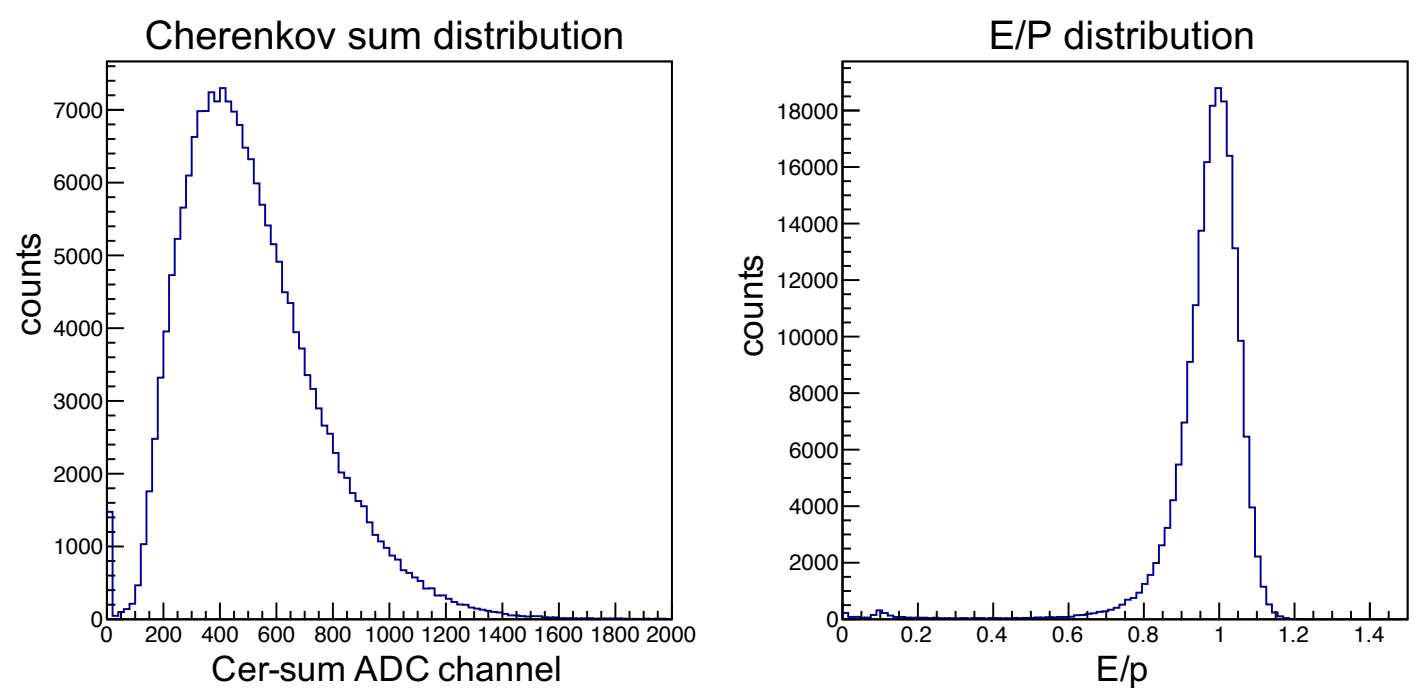

Figure 4.30: The left plot is the Cherenkov sum distribution. The right plot is the $\mathrm{E} / \mathrm{p}$ distribution, electrons are centered around $\mathrm{E} / \mathrm{P} \sim 1$, pions are centered at $\mathrm{E} / \mathrm{P} \sim 0.1$. Only loose acceptance cuts and single track were applied on both plots.

and deposit only a small amount of their energy in the calorimeters. These $\pi^{-}$are found in the region A of the left plot in Figure 4.31. When the $\pi^{-}$'s go through the Cherenkov gas they can produce a knock-on (secondary) electron which can create a Cherenkov signal and fire the trigger. These secondary $e^{-}$produce a small signal in the calorimeters, see the left plot region B in Figure 4.31. In addition with very small probability, the $\pi_{s}^{-}$can produce photons through the channel $\pi^{-} p \rightarrow \pi^{0} n \rightarrow \gamma \gamma$. These photons create a large signal in calorimeter but have no Cherenkov signal, see the left plot region $\mathrm{C}$ in Figure 4.31. The main trigger cut can help to get rid of the events from the region $\mathrm{A}$ and $\mathrm{C}$, as shown as the right plot of Figure 4.31.

For the kinematic settings of E08014, the $\pi / e^{-}$ratio is small, see Table 4.3. In addition, as the Cherenkov is added to the main trigger, removing pions at hardware level, makes the $\pi$ contamination even smaller. To be able to study the $\pi$ rejection efficiency, an efficiency trigger is used to select a $\pi$ sample. We can start with the kinematic setting which has largest ratio $\pi / e^{-}$. If the contamination for this setting is negligible we don't have to worry about other the settings. 

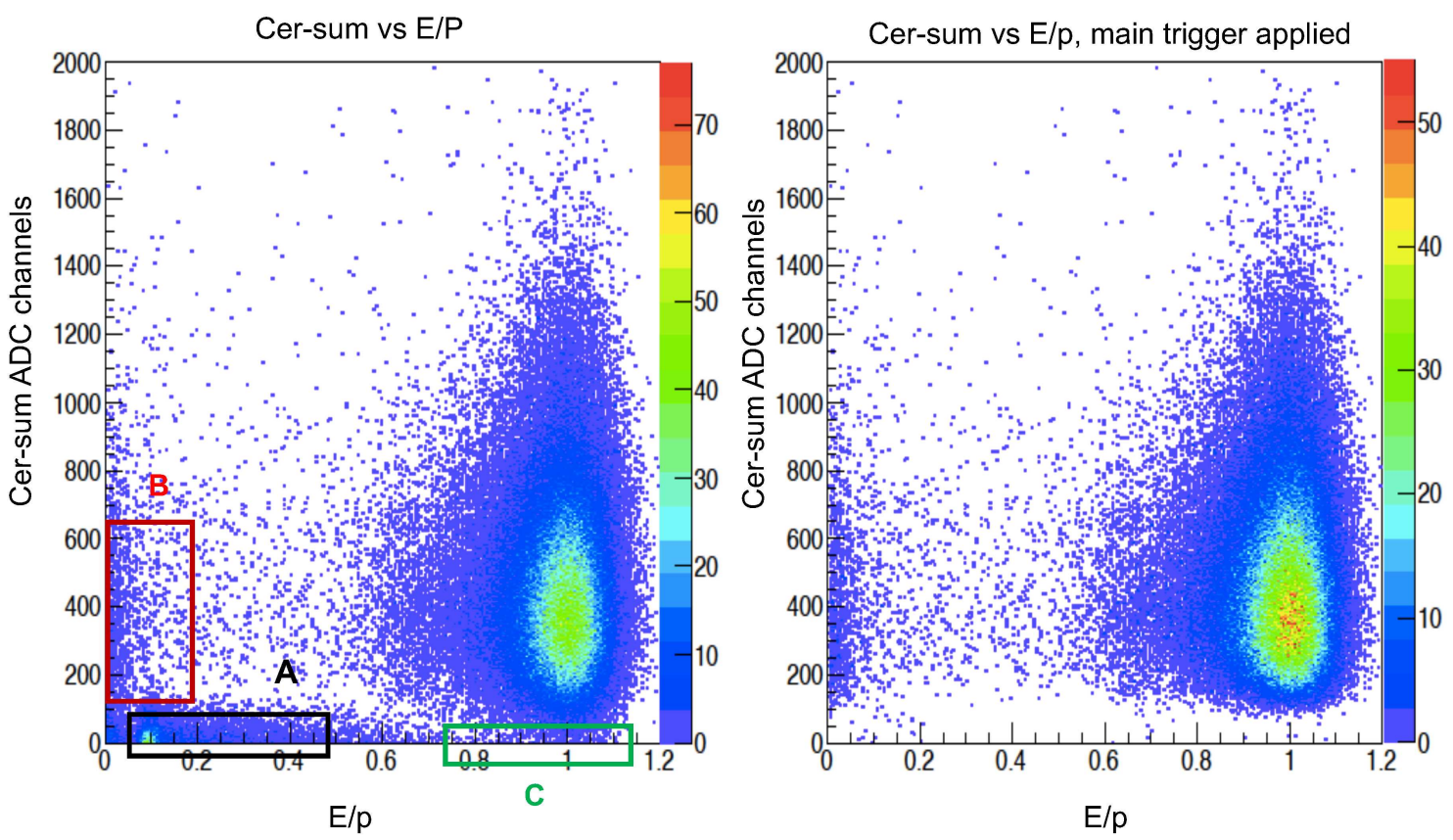

Figure 4.31: The left plot shows the 2D distribution of the Cherenkov sum vs E/p. Only loose acceptance cuts and single track were applied. The region A, these events are pions, they has small energy deposited and no Cherenkov signal.The region B, the events have big Cherenkov signal but small energy deposited in calorimeter. These events are secondary electron. The region $\mathrm{C}$, the events come from photon production. The right plot is the $2 \mathrm{D}$ distribution of the Cherenkov sum vs $\mathrm{E} / \mathrm{p}$ with an additional main trigger cut.

The first step for the PID studies for both cer-sum and E/p cuts is selecting a sample of events with loose cuts on the acceptance, single track and trigger cuts, a sample is shown in Figure 4.32, The subsequent steps in this study will be described in the following sections.

\section{Cherenkov sum cut efficiency}

Determination of the Cherenkov sum efficiency requires a nearly pure electron and pion samples using the calorimeter signal. See Figure 4.33. The electron sample are the events with the large energy deposited in both the pion rejection layer 1 (prl1) and the pion rejection layer $2(\operatorname{prl} 2)$ with $E / p$ is close to one. The pion sample are the events that have little energy deposited in both prl1 and prl2 with $\mathrm{E} / \mathrm{p}$ is close 

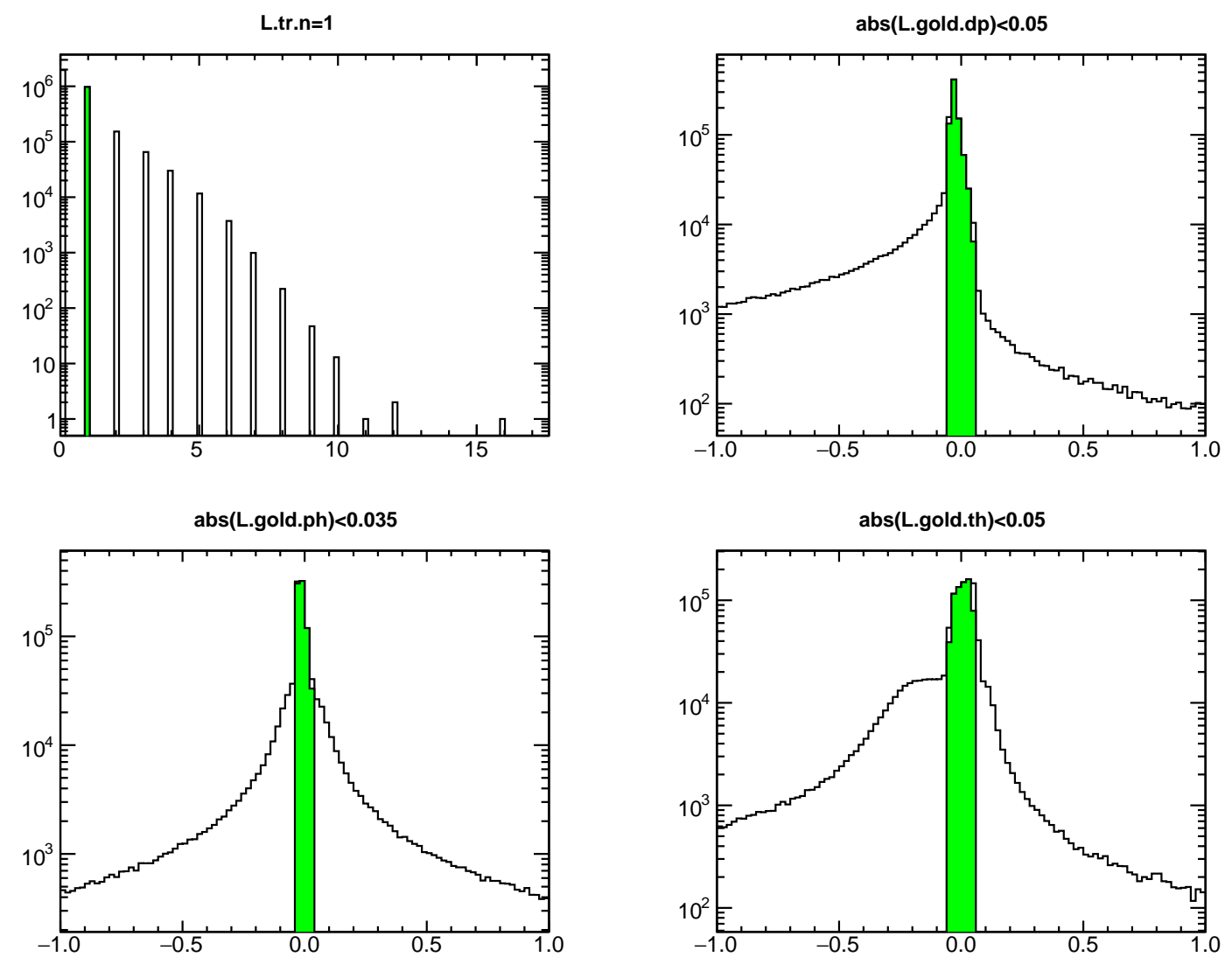

Figure 4.32: Top left plot shows event distribution of the track number (L.tr.n). The top right plot is event distribution for relative momentum variable (L.gold.dp). The left and right bottom plots are event distributions of the in-of-plane (L.gold.ph) angle and out-of-plane (L.gold.th) angle, respectively. Only events pass selection cuts, the single track cut (L.tr.n=1), and loose acceptance cuts (abs(L.gold.dp) $<0.05 \&$ $\operatorname{abs}($ L.gold.ph $)<0.035 \&$ abs $($ L.gold.th $)<0.05)$, are selected. The green region shows the selection cut applied on each variables.

to 0.1. See Figure 4.35. Geographic cuts were used to select the electron and pion samples, see Figure 4.34. Once a sample is selected, we scan (change) the value of the cer-sum cut systematically on the electron and pion samples to see how many electrons remain and how many pions are rejected. The electron efficiency of the cer-sum cut, $\epsilon_{c e r-s u m}^{e^{-}}$, is defined as:

$$
\epsilon_{\text {cer-sum }}^{e^{-}}=\frac{\mathrm{N}_{c a l, c e r-s u m}^{e^{-}}}{\mathrm{N}_{c a l}^{e^{-}}}
$$




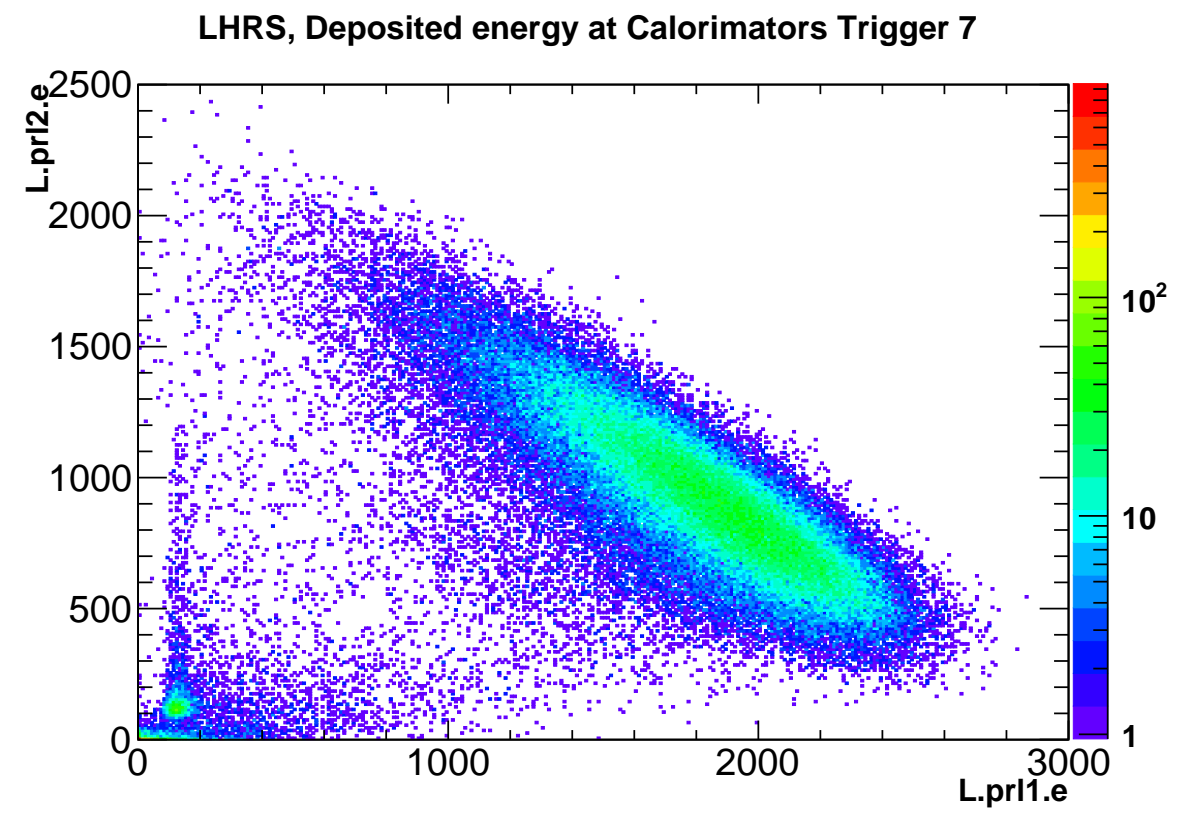

Figure 4.33: The 2D events distribution of prl2 (pion rejection layer 2) vs prl1 (pion rejection layer 1). Loose acceptance, single track, trigger 7 cuts were applied to select the events.

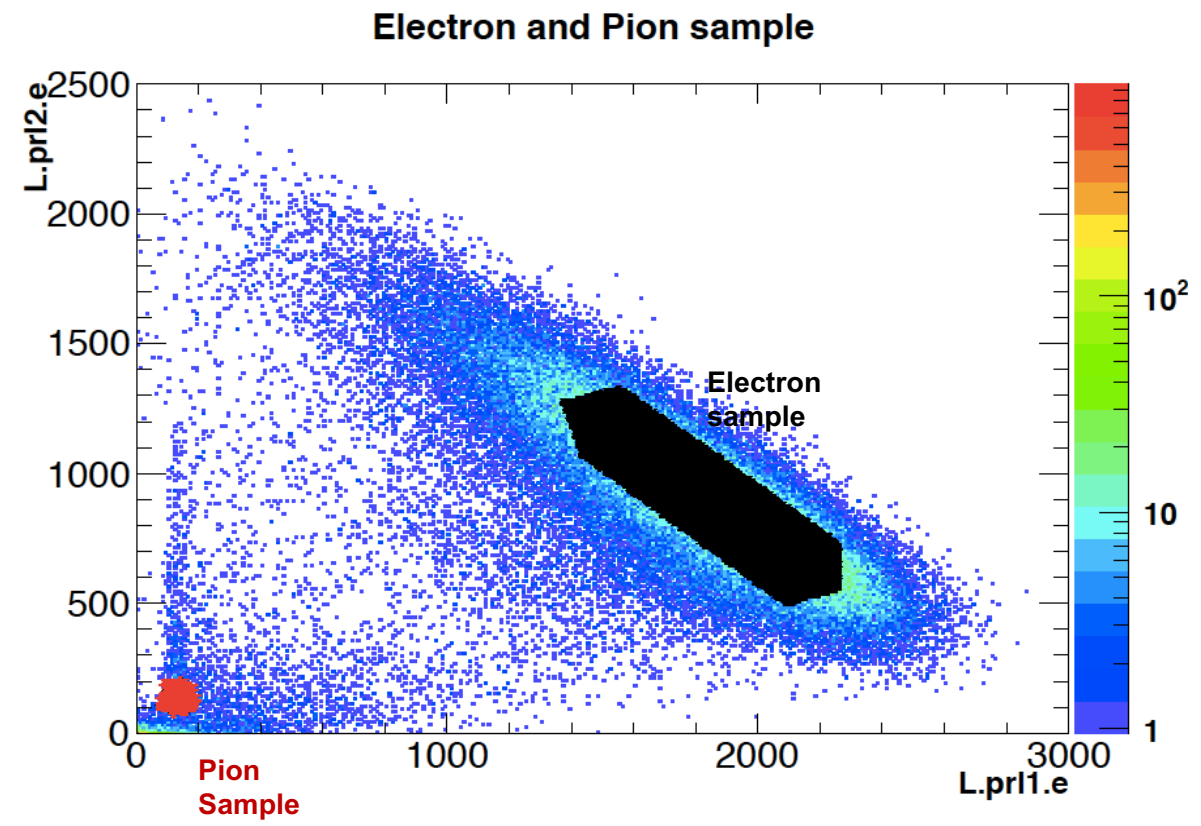

Figure 4.34: The 2D events distribution of the prl2 vs the prl1. The loose acceptance, single track, trigger 7 and geographic cuts were applied to select the electron and pion samples. 


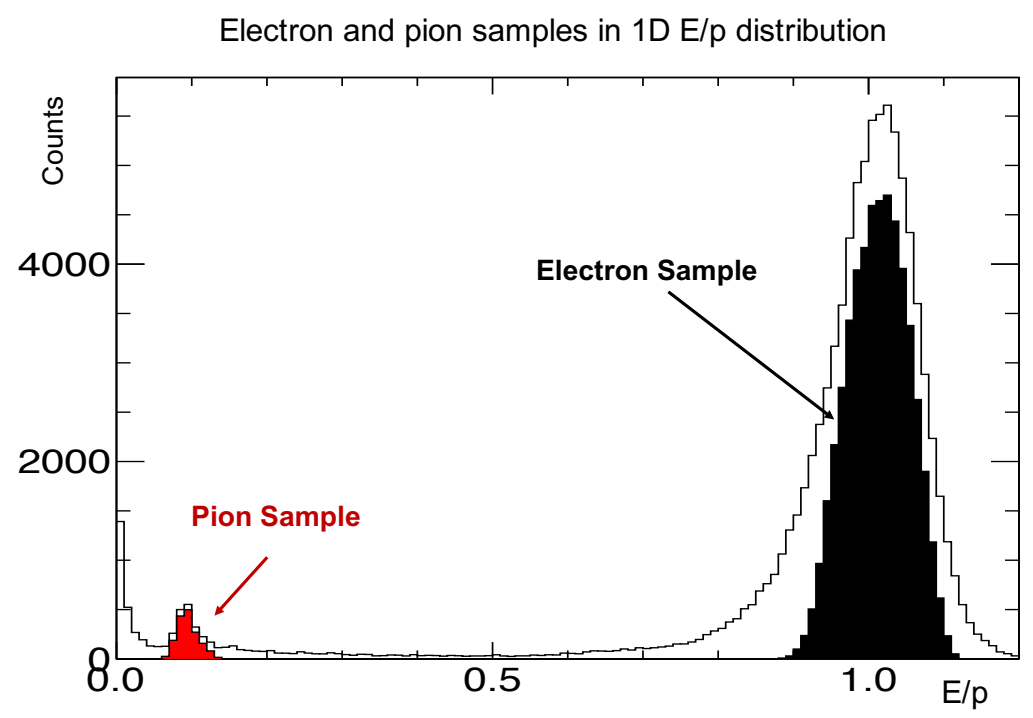

Figure 4.35: The 1D E/p distribution of electron and pion samples. The loose acceptance, single track, trigger 7 and geographic cuts were applied to select the electron and pion samples exactly as in 2D sample plots.

Similarly, the pion rejection efficiency of the cer-sum cut $\epsilon_{c e r-s u m}^{\pi^{-}}$is defined as:

$$
\epsilon_{\text {cer-sum }}^{\pi^{-}}=1-\frac{\mathrm{N}_{c a l, c e r-s u m}^{\pi^{-}}}{\mathrm{N}_{c a l}^{\pi^{-}}}
$$

where $\mathrm{N}_{c a l}^{e^{-}}$and $\mathrm{N}_{c a l}^{\pi^{-}}$are the number of electron and pion in the samples selected using the calorimeter signal, respectively. The $\mathrm{N}_{c a l, c e r-s u m}^{e^{-}}$and $\mathrm{N}_{c a l, c e r-s u m}^{\pi^{-}}$are the number of remaining electrons and pions in the samples after the cer-sum cut. The uncertainty of the efficiency follows the binomial distribution and can be calculated using approximation, expressed as:

$$
\text { Uncertainty }=\epsilon_{\text {cer-sum }}^{e^{-}(\pi)} * \frac{\sqrt{\left(\mathrm{N}_{c a l}^{e^{-}(\pi)}-\mathrm{N}_{c a l, c e r-s u m}^{e^{-}(\pi)}\right)}}{.} \mathrm{N}_{c a l}^{e^{-}(\pi)}
$$

Figure 4.36 shows the cer-sum cut efficiency for both the electron and pion rejection efficiencies. The cer-sum >50 ADC channel is chosen for PID cut where we can keep more than $99 \%$ of electrons and reject more than $96 \%$ of the pions. Note that 


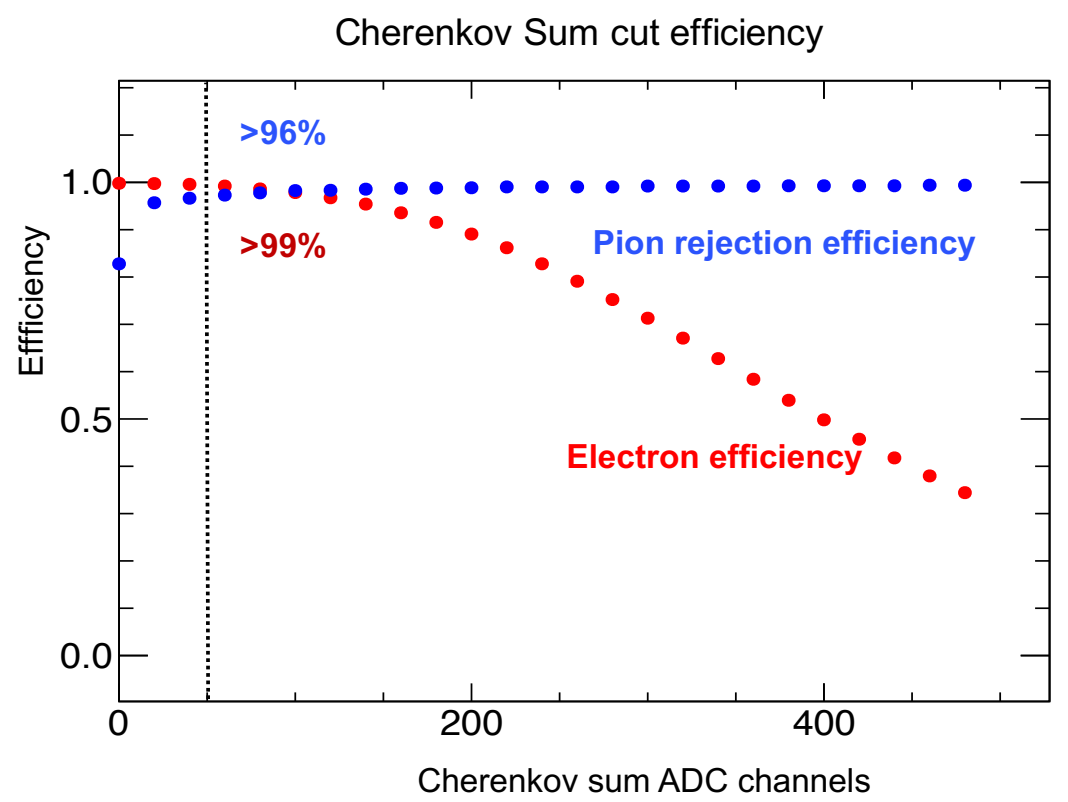

Figure 4.36: The Cherenkov sum cut efficiency for both electron and pion rejection efficiency as function of number of Cherenkov sum ADC channels.

$\pi / e^{-}$ratio is small, the largest one is around $5 \%$, so the remaining pions are less than $0.25 \%$ after the cer-sum cut. With any reasonable E/p cut we can easily bring the remaining pions to well below $0.1 \%$ which is negligible. The pion rejection can be taken to be $100 \%$ without any uncertainty.

\section{$\mathrm{E} / \mathrm{p}$ cut efficiency}

For the E/p cut efficiency study, the procedure is similar to that for the cer-sum. The only the difference is that now we will select the electron and pion samples shown in Figure 4.37 using the Cherenkov sum signal as .

The E/p cut is scanned on the samples to see how many electrons and pions remain after the cut. The electron efficiency and pion rejection efficiency of the E/p cut are defined by

$$
\epsilon_{E / p}^{e^{-}}=\frac{\mathrm{N}_{c e r, E / p}^{e^{-}}}{\mathrm{N}_{c e r}^{e^{-}}} \quad \text { and } \quad \epsilon_{E / p}^{\pi^{-}}=1-\frac{\mathrm{N}_{c e r, E / p}^{\pi^{-}}}{\mathrm{N}_{c e r}^{\pi^{-}}}
$$



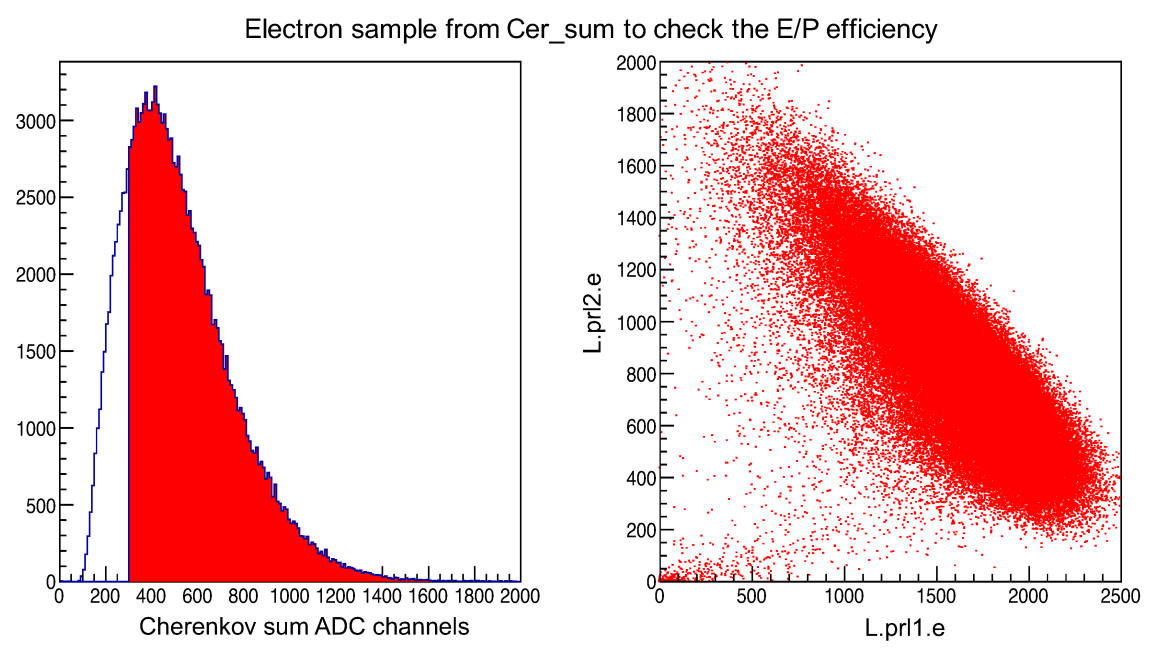

Figure 4.37: The electron sample using the Cherenkov sum to calculate the E/P efficiency. The cuts applied are a trigger cut, the loose acceptance cut and cer-sum $>300$ ADC channels. The right plot show (prl2 vs prl1) distribution of selected event on the left plot.

where $\mathrm{N}_{c e r}^{e^{-}}$and $\mathrm{N}_{c e r}^{\pi^{-}}$are the number of electrons and pions in the samples selected using the Cherenkov signal, respectively. The $\mathrm{N}_{c e r, E / p}^{e^{-}}$and $\mathrm{N}_{c e r, E / p}^{\pi^{-}}$are the number of remaining electrons and pions in the samples after the $\mathrm{E} / \mathrm{p}$ cut.

As discussed in Section 4.5.3, only the electron efficiency is calculated for this experiment and the $p i$ contribution can be ignored. Figure 4.38 shows the electron efficiency of $\mathrm{E} / \mathrm{p}$ cut as a function of $\mathrm{E} / \mathrm{p}$. The cut $\mathrm{E} / \mathrm{p}>0.5$ is chosen as calorimeter PID cut which allows a retention of more than $99 \%$ of the electrons while insuring make we have good pion rejection in combination with the cer-sum cut. Table 4.3 is the summary of cer-sum $>50$ ADC channel and E/p $>0.5$ electron efficiency cut for different kinematic settings.

As a summary for PID efficiency cut, the cut cer-sum> 50 ADC channels has an efficiency of $99.5 \%$ for every run, $0.3 \%$ normalization uncertainty and $0.1 \%$ uncertainty point to point. The $\mathrm{E} / \mathrm{p}>0.5$ cut has an efficiency of $99.6 \%$ for every run, $0.3 \%$ normalization uncertainty and $0.1 \%$ uncertainty point to point. These uncertainties are applied to the absolute cross section. For the cross section ratios only 


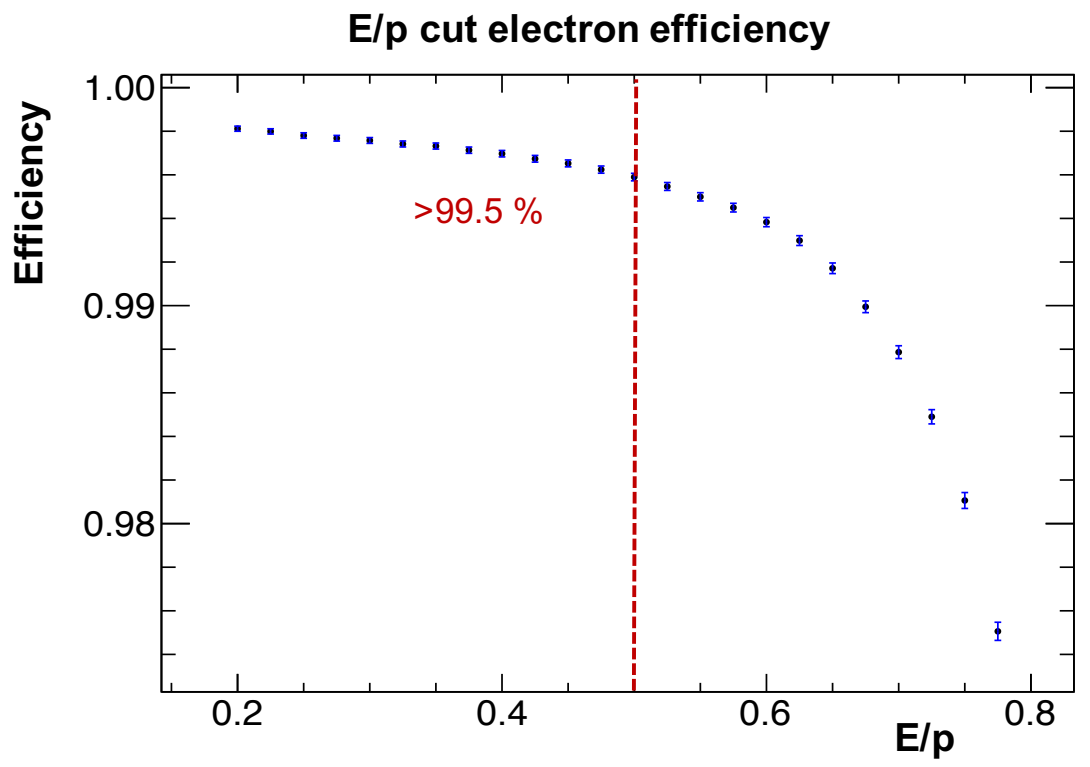

Figure 4.38: The E/p electron efficiency as function of number of $\mathrm{E} / \mathrm{p}$.

Table 4.3: Particle Identification efficiency summary

\begin{tabular}{||c|c|c|c|c|c||}
\hline \hline Kin & $\pi / e^{-}(\%)$ & Cer-sum cut & $\epsilon_{\text {cer-sum }}^{e^{-}}(\%)$ & $\mathrm{E} / \mathrm{p}$ cut & $\epsilon_{E / p}^{e^{-}}(\%)$ \\
\hline kin5.0 & 4.7 & 50 & 99.4 & 0.5 & 99.7 \\
\hline kin5.05 & 3.3 & 50 & 99.4 & 0.5 & 99.7 \\
\hline kin5.1 & 3.7 & 50 & 99.6 & 0.5 & 99.5 \\
\hline kin3.1 & 1.0 & 50 & 99.4 & 0.5 & 99.6 \\
\hline kin3.2 & 1.0 & 50 & 99.5 & 0.5 & 99.4 \\
\hline kin4.1 & 1.7 & 50 & 99.5 & 0.5 & 99.6 \\
\hline kin4.2 & 2.5 & 50 & 99.4 & 0.5 & 99.1 \\
\hline
\end{tabular}

0.1\% uncertainty point to point applied because the cancelation, Ref. [76].

\subsubsection{Tracking Efficiency}

The VDCs are responsible for the tracking information in the HRSs. This tracking information is used to reconstruct the position and angle of the particle trajectory which is required to determine the kinematic variables for each event. A quick check on the VDCs wires efficiency is performed to make sure that the VDCs work properly at the hardware level. Then a detailed discussion on the tracking efficiency determination 
will be provided.

\section{Wire efficiency}

For a given wire plane, the wire efficiency can be estimated by scanning through every wire in that plane for every event. A wire is efficient if it fires when it is located between two wires which have fired [65]. The efficiency for each wire is obtained using the following expression:

$$
\text { Efficiency }=\frac{k}{k+\lambda}
$$

where $k$ and $\lambda$ are the number of times that a given wire fires or doesn't fire, respectively. Figure 4.39 shows the wire efficiency as function of wire number for each wire plane U1, V1, U2, V2 for the two VDCs. The wire efficiency is high and looks reasonable for all wires planes. This gives confidence that the VDCs wires work properly.

\section{Single track efficiency}

Simply stated, the tracking efficiency is defined as a percentage of good events for which a good track is reconstructed in the VDC $69,77,78$. First we will describe how the track is reconstructed in VDC so that we can define whether a track is good or not. When a charged particle passes through the VDC, it ionizes the gas. The electrons created by the ionization drift to the wire along the electric field line which has the shortest time path, schematically as arrows in Figure 4.40. The nominal trajectory can fire 4 to 6 wires [59] which define a cluster. A cluster consists of hits with consecutive wire numbers and are allowed to have gaps of one wire without a hit to account for any inefficiency. Figure 4.40 shows a typical 5 cell cluster.

The drift times $t_{i}$ from the trajectory to different wires are measured by TDCs then converted to drift distances $d_{i}$, the dashed lines in Figure 4.40. After the drift distances are determined, a linear fit of drift distance vs. wire position is performed to 
Left arm U1 efficiency

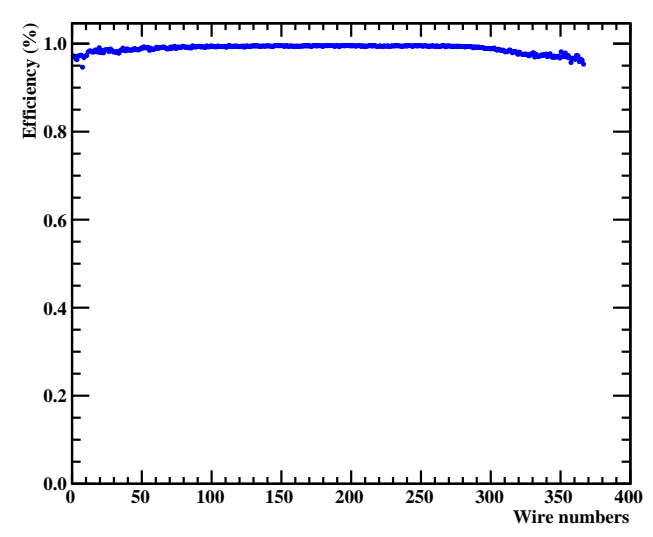

Left arm U2 efficiency

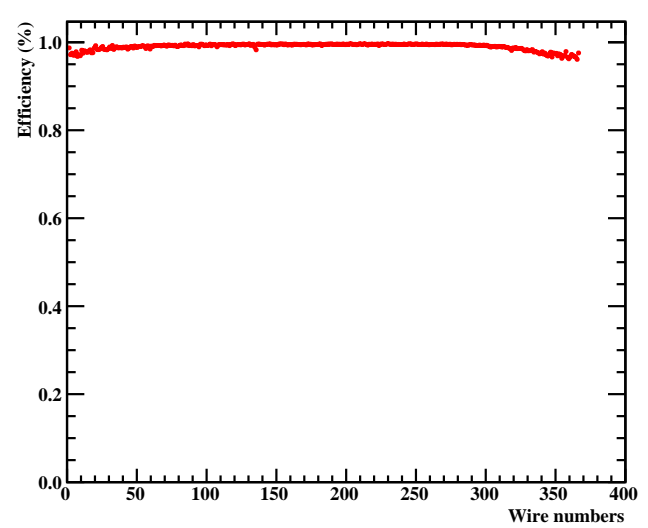

Left arm V1 efficiency

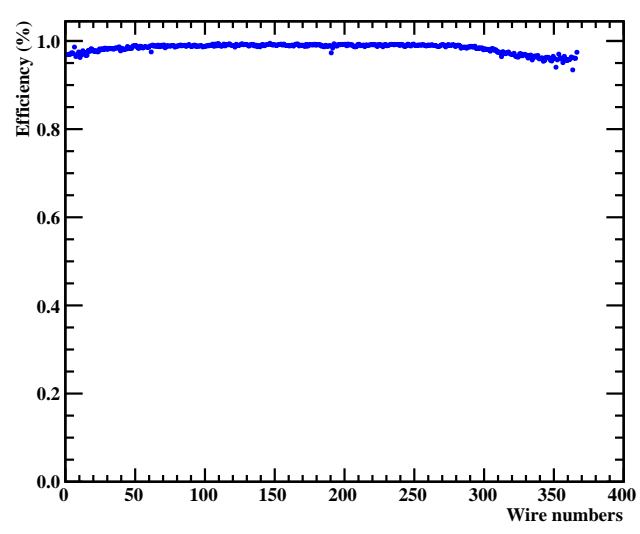

Left arm V2 efficiency

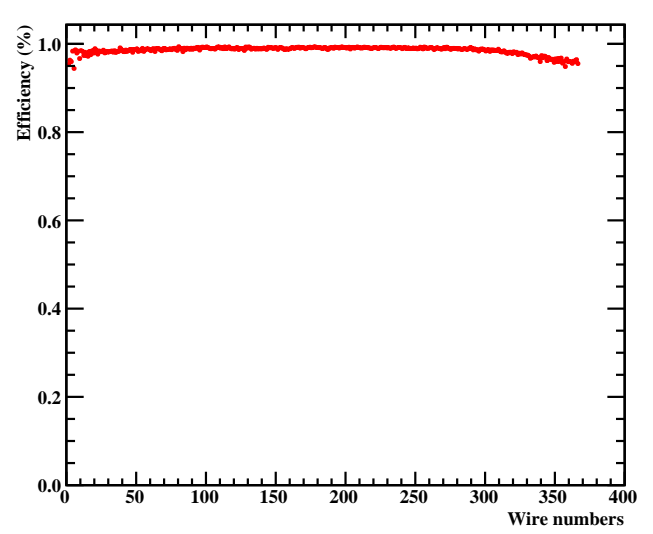

Figure 4.39: The wire efficiency as function of wire number for the 4 wire planes of the two VDCs. The top two plots are for U1, V1 wire planes. The two bottom plots are for $\mathrm{U} 2, \mathrm{~V} 2$ wire planes.

determine the local cross-over point $U(V)_{1,2}$ and the local angle of the track $\Theta_{U(V)_{1,2}}$ for each wire plane. While the $\Theta_{U(V)_{1,2}}$ determination depends on the tracking algorithms strongly, the $U(V)_{1,2}$ can be determined with high precision and is insensitive to the tracking algorithms as well as the local track angle information [65, 80]. This is the advantage of the VDCs. The global track angles $\Theta_{U}$ and $\Theta_{V}$ are obtained using measurements from the two track cross-over points in the two VDCs as expressed in Equation 4.24, where $d$ is the distance between two $U$ or $V$ wire planes. See Figure 4.41 for context.

$$
\tan \left(\Theta_{U, V}\right)=\frac{U(V)_{2}-U(V)_{1}}{d} .
$$




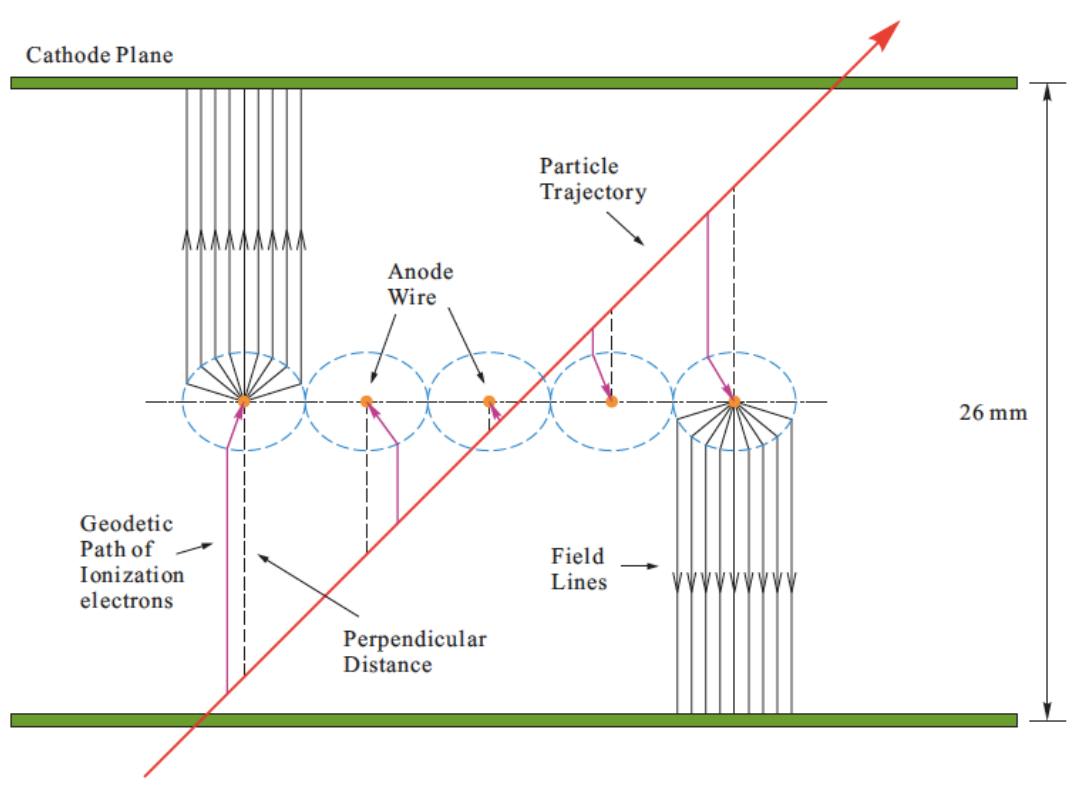

Figure 4.40: A cluster with 5 cells in a wire plane in the VDCs [65, 79].

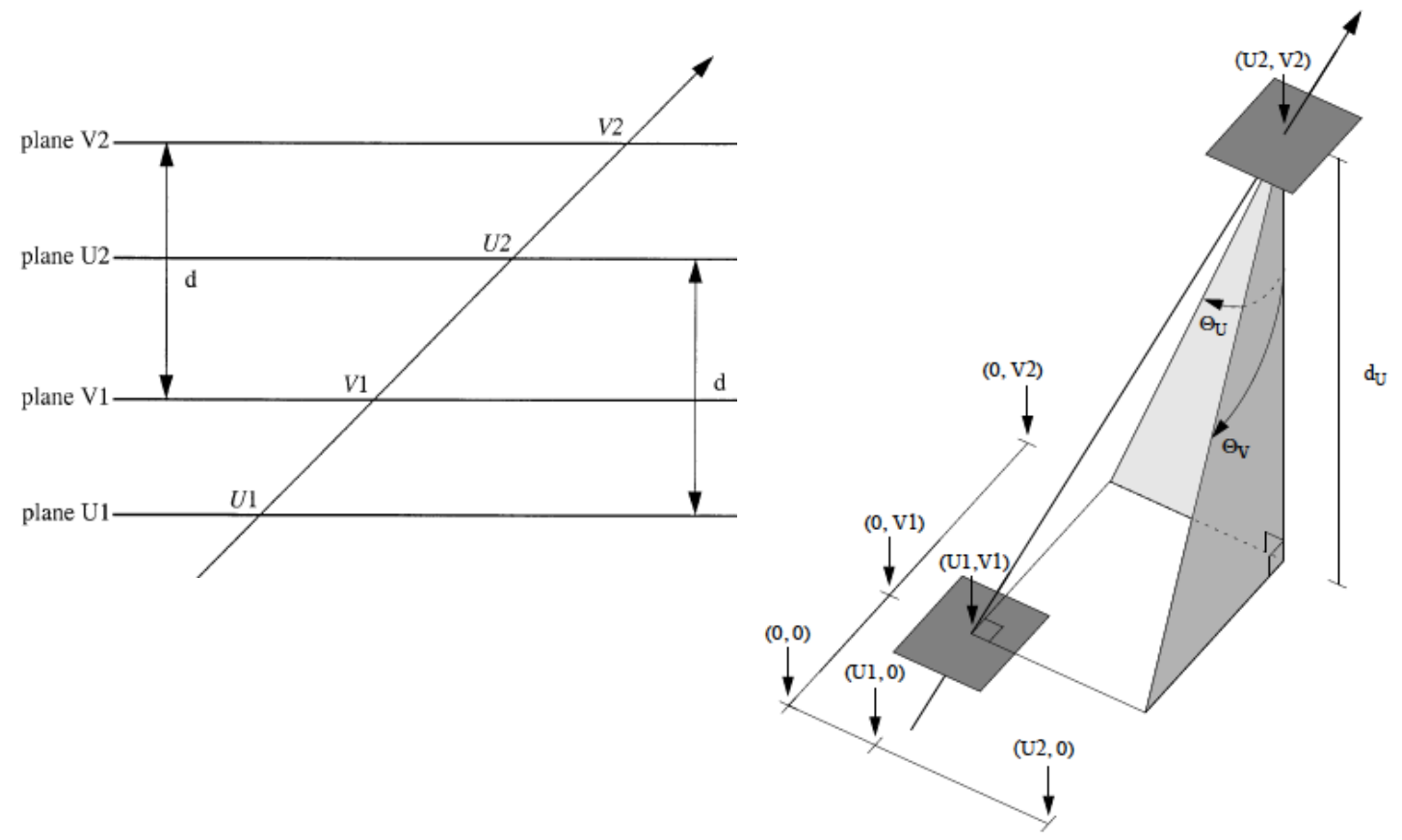

Figure 4.41: The global track using two cross-over points from two VDC chamber, Figure from Ref. [65].

Once the global track is reconstructed, one more iteration is needed to recalculate the drift distance and then a new linear fit is performed to get the new local cross- 
over and local angle of the track. By following this approach, the global track angle has good accuracy [80]. The number of tracks depend on the number of the clusters on each wire plane. As a result, the number of tracks can be zero, single track or multiple tracks. The main reason for zero track events is one of the wire plane has no cluster. The reason for multiple tracks is at least one of the VDC planes has more then one cluster. The multiple clusters may occur for several reasons such as noise or true multiple tracks from the target [59]. Figure 4.42 shows the total number of clusters in the wire plane $\mathrm{U}$ and $\mathrm{V}$ for each VDC for zero track event (left plot) and multiple track event (right plot).
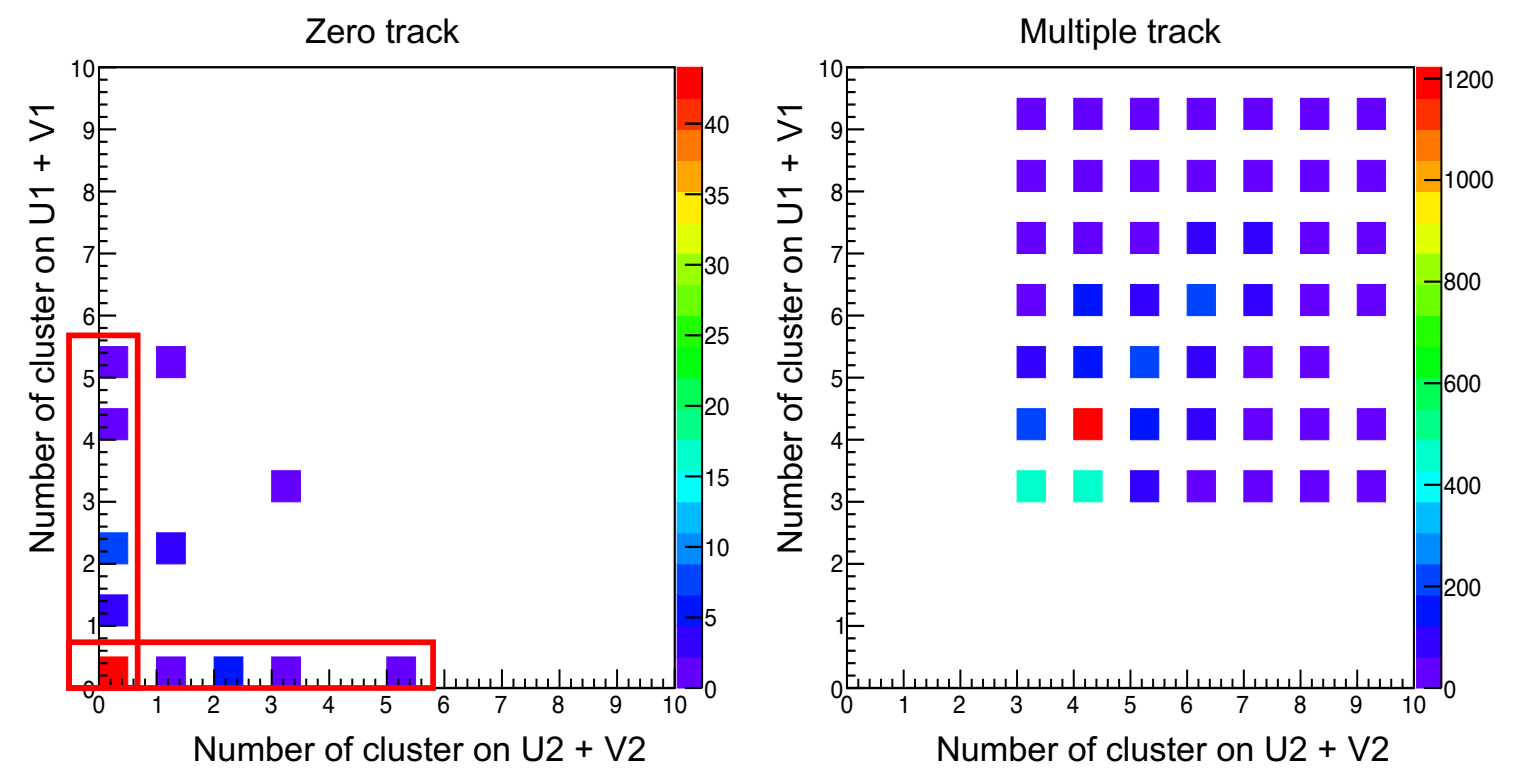

Figure 4.42: Total number of cluster from wire plane U, V for each VDC chamber. The left plot is for the Zero tracks, showing that at least one of wire planes has no clusters. The right plot is for Multiple tracks, showing that at least one of wire planes have more than one cluster.

In this analysis only single tracks are considered as good tracks, so the track efficiency is the single track efficiency. Only single track events are used in the analysis to get the yield, so the yield is divided by this efficiency to account for the zero and multiple track events. In the first step, we need to evaluate the fraction of zero track 
events. To calculate this efficiency, the good electron sample is selected using the trigger cut, PID cut (cer-sum $>300$ and $E / P_{0}>0.8$ ). Because we are calculating

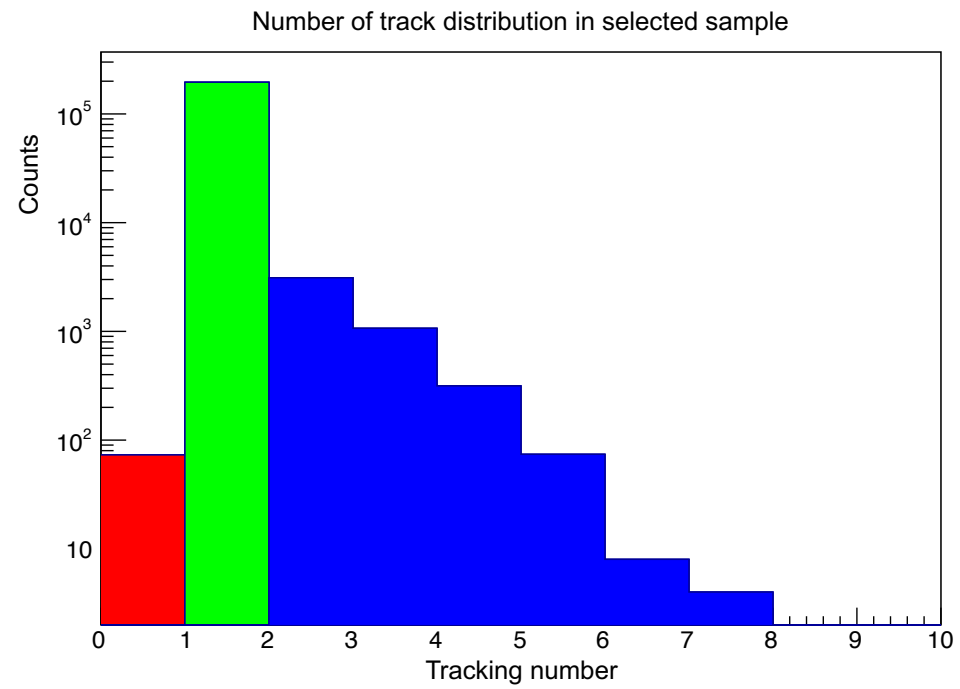

Figure 4.43: The track number distribution in the selected sample for tracking efficiency calculation. The plot is for the target ${ }^{12} \mathrm{C}$ and kin5.0

the tracking efficiency, no tracking information can be used when we select sample otherwise a bias is introduced. For the PID cut, the total deposited energy in the calorimeter is divided by the central momentum $P_{0}$, not the measured momentum of the event. this done because measured momentum of each event is obtained by using the reconstructed relative momentum what requires the tracking information. Then the zero track efficiency, $\epsilon_{\text {zero track }}$, can be calculated as following expression.

$$
\epsilon_{\text {zero track }}=\frac{N_{0}(\text { Trigger }, \text { PID }, \text { Track }=0)}{N_{\text {sample }}(\text { Trigger }, \text { PID })}
$$

The zero track events in the selected sample have a good trigger and pass PID cuts which means that it is potentially by a good electron and should have at least one track. The fact that there is no track in the VDC indicates that the VDC was inefficient for that event. The zero track efficiency corrects this. In this experiment the zero track fraction was very small, as can be seen in Table 4.4. Next the single 
track and multiple track efficiencies can be calculated. The concern in this study is that some of the events are lost not because of inefficiency but because of the background. For example, there might be an event in the corner of the VDCs that missed one plane and is outside the nominal acceptance or an event scatters into the detector from the Q3 exit. We don't want to correct for these because they are not a part of real inefficiency. A new sample is selected using the trigger, PID cuts and loose acceptance cuts. The acceptance cuts help to get rid of irrelevant events. Then the single track efficiency is the fraction of events in this electron sample that have one track. See Equation 4.26, A similar calculation is applied for the multiple track efficiency. Note that in the new sample there are no zero track events, so the total efficiency of single tracks and the multiple tracks is $100 \%$.

$$
\epsilon_{\text {single track }}=\frac{N_{1}(\text { Trigger }, \text { PID }, \text { loose acceptance cuts }, \text { Track }=1)}{N_{\text {sample }}(\text { Trigger }, \text { PID }, \text { loose acceptance cuts })}
$$

Multiple track events that may have multiple good electrons are discarded by the single track cut in the analysis. The single track efficiency can correct for these lost electrons as well [77]. The tracking efficiency depends on the event rate, so we need to calculate this efficiency for different kinematics. In addition, the pre-scaler for each run in the same kinematic can change and this requires the an efficiency calculation run by run in the analysis. Table 4.4 shows the zero, single and multiple track efficiency for a pre-scale 1 run in each kinematic setting for ${ }^{12} \mathrm{C}$ target as an example.

The uncertainty of the single track efficiency follow the binomial distribution because the event can either have single track or not. Because of the variance in the single track efficiency from different kinematics, in addition, the cross section analysis will use tighter acceptance cuts which means that we may lose more good events. In the end a single track efficiency of $98.5 \%$ is applied to every run with a $1 \%$ normal- 
Table 4.4: Tracking efficiency for zero, single, multiple track for each kinematic

\begin{tabular}{||c|c|c|c|c|c||}
\hline \hline Kin & Target & Run & Zero & Single & Multiple \\
\hline kin5.0 & ${ }^{12} \mathrm{C}$ & 3634 & 0.03 & 99.2 & 0.8 \\
\hline kin5.05 & ${ }^{12} \mathrm{C}$ & 4209 & 0.05 & 98.9 & 1.1 \\
\hline kin5.1 & ${ }^{12} \mathrm{C}$ & 3825 & 0.09 & 98.6 & 1.4 \\
\hline kin3.1 & ${ }^{12} \mathrm{C}$ & 3664 & 0.08 & 98.2 & 1.8 \\
\hline kin3.2 & ${ }^{12} \mathrm{C}$ & 3663 & 0.13 & 98.0 & 2.0 \\
\hline kin4.1 & ${ }^{12} \mathrm{C}$ & 4163 & 0.08 & 98.5 & 1.5 \\
\hline kin4.2 & ${ }^{12} \mathrm{C}$ & 3692 & 0.15 & 98.0 & 2.0 \\
\hline
\end{tabular}

ization uncertainty and $0.3 \%$ point to point uncertainty [76]. 


\subsection{Acceptance Study}

This section will give an introduction to the acceptance study for the Hall A HRS. The acceptance is defined as the probability that a particle will make it through the spectrometer. The three ingredients needed for the acceptance study are the magnetic optics, the simulation and a cross section model. The optics was discussed in Section 4.4, the following sections present the discussion on the simulation and the model. The comparison between simulation and data for reconstructed target variables help to define how well the acceptance is modeled. In the end, the main goal of this acceptance study is to determine a set of cuts for the target variables (acceptance cuts) in the cross section analysis.

\subsubsection{Simulation (SAMC)}

The Single Arm Monte Carlo (SAMC) simulation package is used in this analysis, see Ref. [81]. The SAMC was originally developed in Fortran then converted to $\mathrm{C}++$ by Huan Yao [82 and modified by Z. Ye [54]. The working principle of this simulation can be summarized as the following steps:

- Generate events: Uniform generation of target quantities including in-plane angle $\phi_{t g}$, out-plane angle $\theta_{t g}$, relative momentum $d p_{t g}$ and the beam energy following a Gaussian distribution while adding smearing of the energy resolution.

- Forward transportation: After events are generated at the target, the forward matrix transports the events from the target to the focal plane. This transportation is step by step through every aperture and checks are made to whether a particle pass through or get stopped. In the SAMC, only 5 different locations are checked; the check locations and the distance from the target following the trajectory path are listed in Figure 4.44. The size of each aperture used in SAMC are given in Table 4.5. The apertures location is matched 
to the technical drawing information [83]. Only events which pass through all apertures are recorded as detected events at the VDCs. At that point VDC smearing is added.

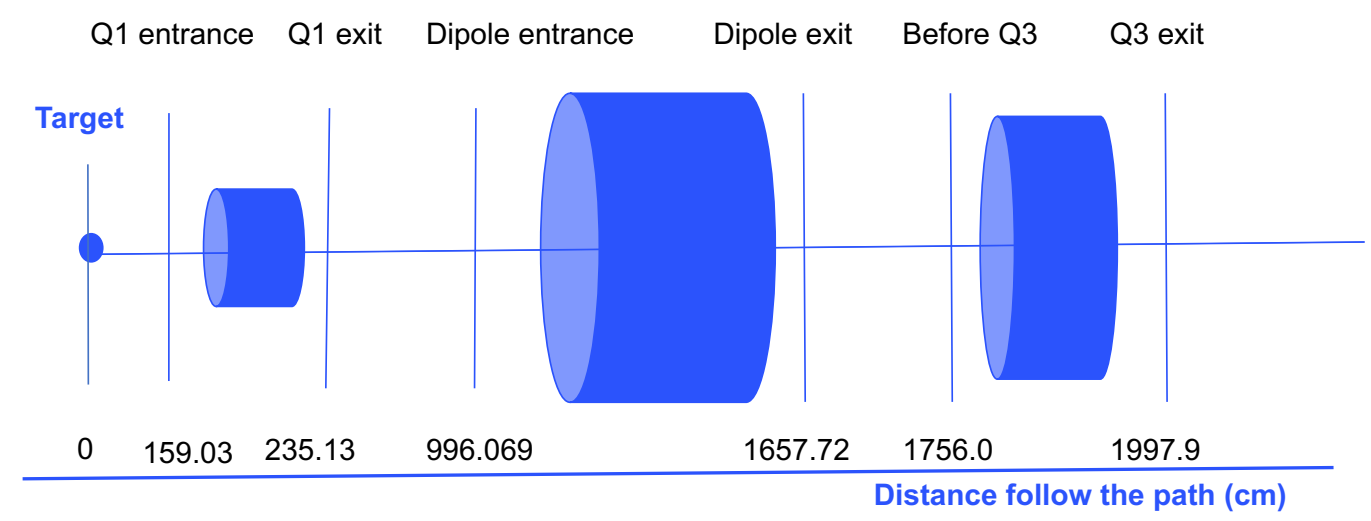

Figure 4.44: The location of aperture that are checked in the SAMC. The distance is the particle path from target in $\mathrm{cm}$.

Table 4.5: SAMC apertures check list

\begin{tabular}{||c|c|c|c||}
\hline \hline Aperture name & Location $(\mathrm{cm})$ & Aperture size check $(\mathrm{m})$ & notice \\
\hline Q1 exit & 253.13 & $\sqrt{x^{2}+y^{2}}<0.1492$ & Q1 radius \\
\hline D Entrance & 996.069 & $|y|<0.125 *(1-1.25 * x / 8.4)$ & Shape of D \\
\hline D exit & 1657.2 & $|y|<0.125 *(1-1.25 * x / 8.4)$ & Shape of D \\
\hline Q3 entrance & 1756 & $\sqrt{x^{2}+y^{2}}<0.3$ & Q3 radius \\
\hline Q3 exit & 1997.9 & $\sqrt{x^{2}+y^{2}}<0.3$ & Q3 radius \\
\hline
\end{tabular}

- Backward transportation: The backward matrix is used to reconstruct the detected events at VDC back to the target.

The forward and backward matrix were generated by J. Lerose based on the SNAKE program [84]. The SAMC version used in this analysis is an unweighted simulation. This means that no cross section model is included in the simulation of events. The inputs required for this simulation are the beam energy, scattering angle, 
central momentum, target makeup and all offset information from the spectrometer, beam line and the target. A detailed pointing study is performed in the next section to provide the input information for the simulation. An external cross section model XEMC [85] is used to add the weighting factor for each reconstructed event from simulation before they can be compared to the data. The structure of simulation package can be found in Figure 4.45 .

Simulation SAMC package Structure

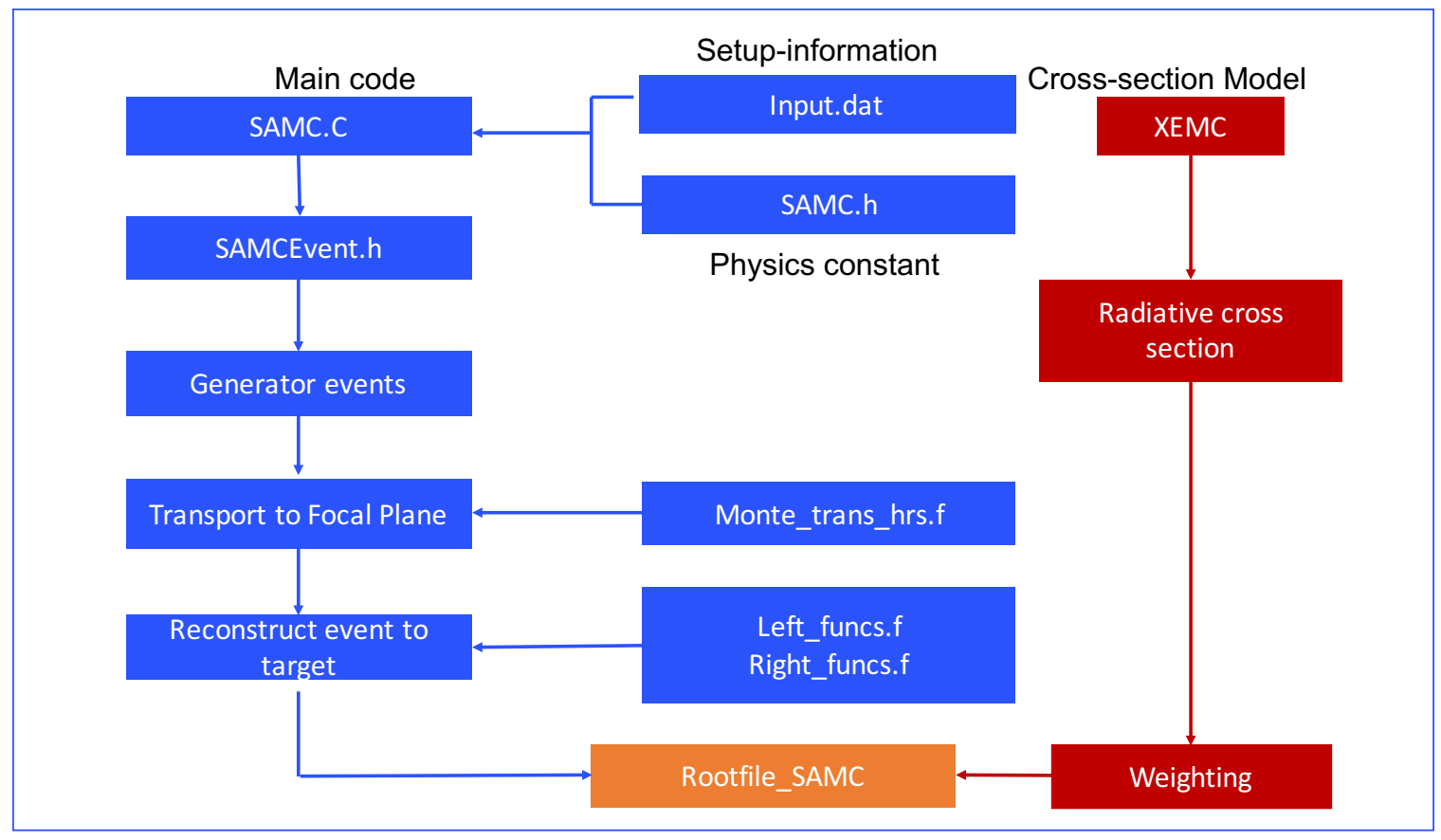

Figure 4.45: The SAMC package structure.

\subsubsection{Pointing Study}

The goal of the pointing study is the determination of the central angle of the spectrometer and the spectrometer offsets 86, 87. These are required to calculate the vertex variables and physics quantities for the simulation as well. To be able to do this study we need to have survey information. But no survey was performed during the E08014. The survey data from E08-008 that was running in the same period of 
time are used to complete this study [88. The process is described in detail below.

\section{Spectrometer mis-pointing}

In the ideal situation the spectrometer movement is only the rotation around the center of the hall, and the central ray of the spectrometer points to the hall center. In reality, the spectrometer has translational movement that causes the central rays to miss the hall center in both the horizontal and vertical directions. The horizontal offset refers to the spectrometer mis-pointing or Spec-off. Figure 4.46 describes the layout with the following definitions:

where:

Convention for the spectrometer miss-pointing

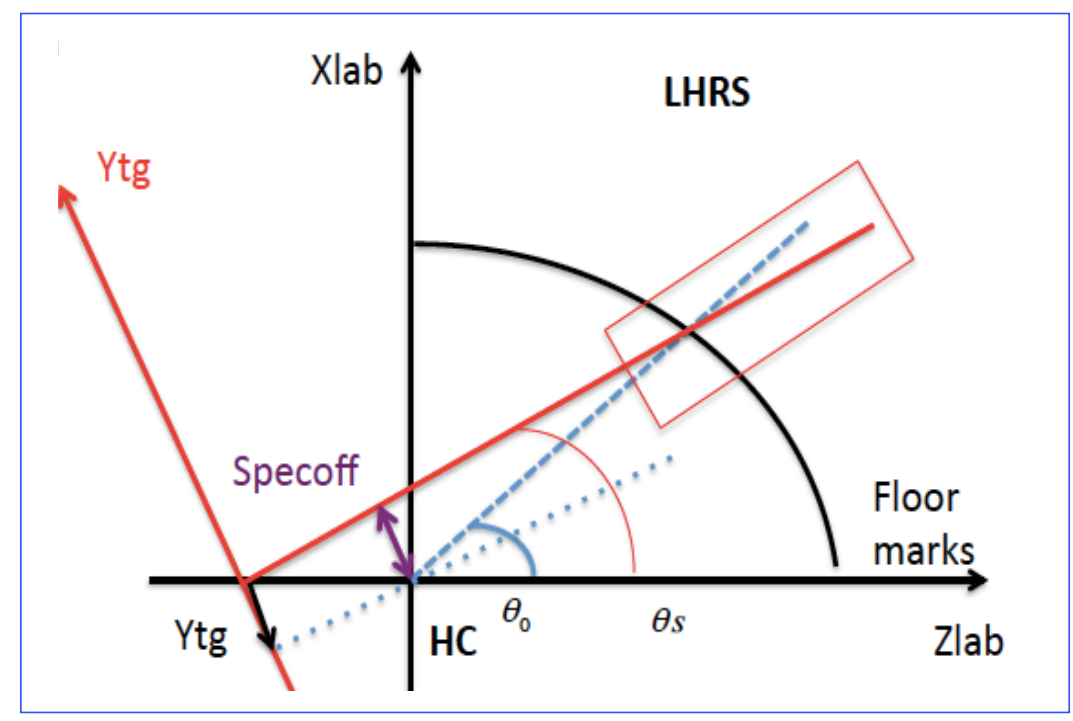

Figure 4.46: The convention for mis-pointing study in the Lab and the target coordinator system.

- HC : Hall center

- $\theta_{0}$ : The central angle

- Spec-off: The spectrometer offset

- $\mathrm{R}$ : Radius on the floor mark and $\mathrm{R}=8.458 \mathrm{~m}$ 
- $\theta_{s}$ : The corrected angle: $\theta_{s}=\theta_{0}-\Delta \theta$ where $\Delta \theta=$ spec-off $/ \mathrm{R}$

The spectrometer offset is not reproducible. It means that even we move spectrometer back to the same angle location at a different time the value of spectrometer offset (Spec-off) will be different. The survey can provide the information necessary to find both the spectrometer offset and the vertical offset. Unfortunately, surveys take a lot of time and surveys are not done for every angle setting; usually they are done only for optics data taking.

For the settings for which we don't have survey data we can use $\mathrm{Y}_{t g}$ reconstructed from what is called "the pointing data" on the single ${ }^{12} \mathrm{C}$ target to calculate the spectrometer offset. But we need to have a reasonable optics matrix before we can do this calculation. In ideal situation, everything is perfect we only have the spectrometer offset (see Figure 4.46) the calculation proceeds through the following expression:

$$
\text { Ytg }=\text { spec-off. }
$$

In fact we have other offsets, the beam offset and the target offset. They all contribute to the offset on $Y_{t g}$. To be able to derive a solution we must consider the contribution from each offset individually.

- Beam X offset: In the ideal case the beam direction goes through the HC otherwise we have a beam offset. But only the beam X offset effects the $\mathrm{Y}_{t g}$ and spec-off because they are all in horizontal direction. In the case everything else is perfect we only have beam X offset (see Figure 4.47) and its contribution to $\mathrm{Y}_{t g}$ is:

$$
\mathrm{Y}_{t g}=\operatorname{Beam} \mathrm{X} * \cos \left(\theta_{s}\right)
$$

- Target offset: In the ideal case the target is installed at the HC otherwise we have a target offset. In case of everything being perfect only the target has an 
offset (see Figure 4.48) and its contribution to the $\mathrm{Y}_{t g}$ is:

$$
\mathrm{Y}_{t g}=- \text { Target-off } * \sin \left(\theta_{s}\right)
$$

- Optics offset: The optics matrices are not perfect so we have the offset left from the optics reconstruction which contributes to the $\mathrm{Y}_{t g}$ as well.

$$
\mathrm{Y}_{t g}=\text { optic-off. }
$$

The beam offset contribution on the Ytg

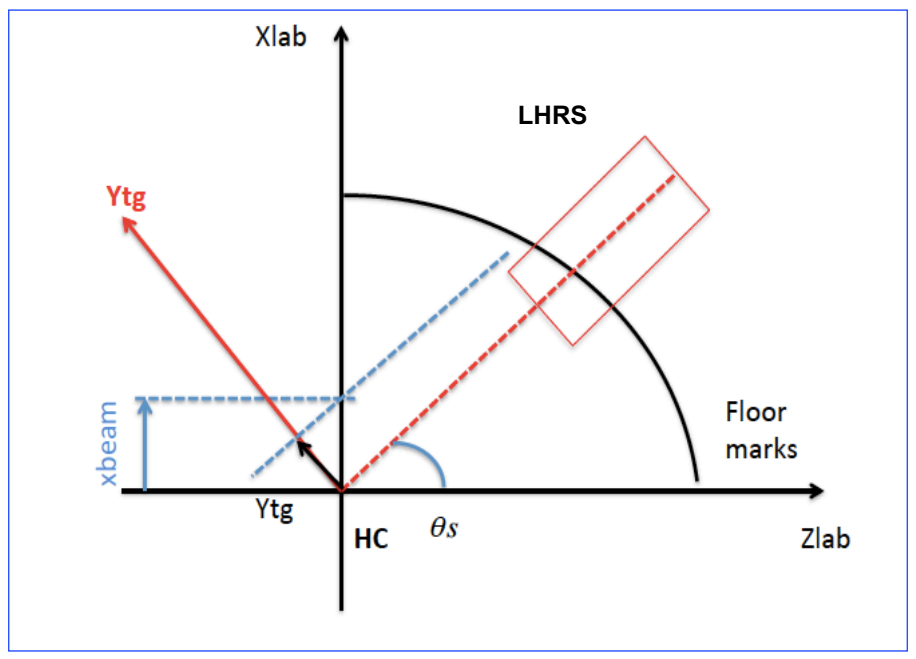

Figure 4.47: The plot shows the contribution of the beam offset in $\mathrm{X}$ direction to the $\mathrm{Y}_{t g}$.

In the total we have an equation for $\mathrm{Y}_{t g}$ including all offset contributions:

$$
\text { Ytg }=\text { spec-off }+ \text { Beam } \mathrm{X} * \cos \left(\theta_{s}\right)-\text { Target-off } * \sin \left(\theta_{s}\right)+\text { optic-off }
$$

The $\mathrm{Y}_{t g}$, beam-X offset can be obtained from data while the spec-off and target-off come from the survey. A survey is needed to determine optics-off and then to calculate the spec-off for other settings of spectrometer. 
The target offset contribution to the Ytg

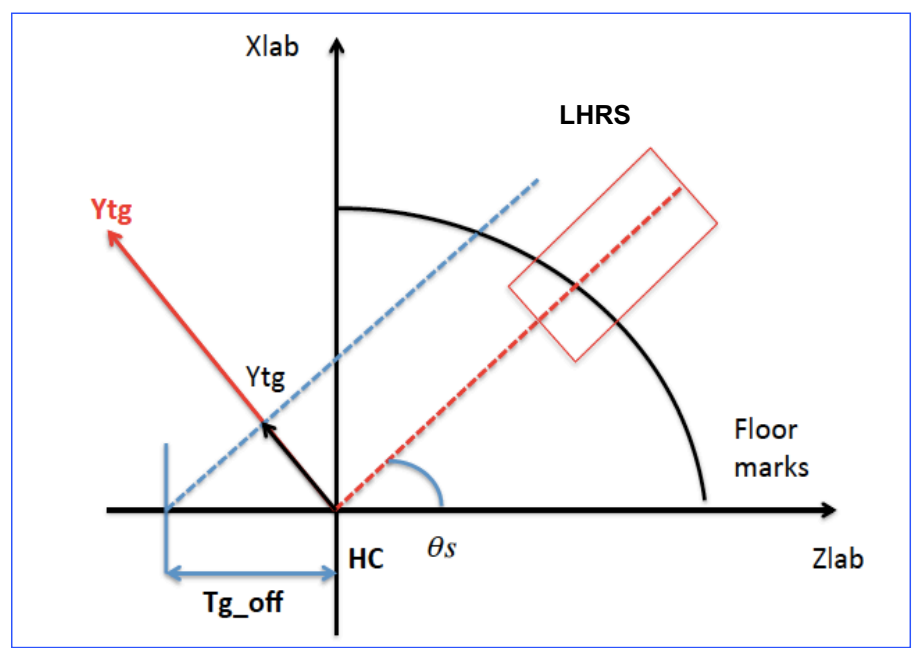

Figure 4.48: The plot shows the contribution of the target offset to the $\mathrm{Y}_{t g}$.

\section{Spectrometer survey}

E08014 was running with 3 other experiments in the same period of time [89]. A survey was performed at beginning of the period and this information is listed in Table 4.6 88. Run 1212 which was taken right after the survey using the multiple

Table 4.6: Survey results for Spectrometer mis-pointing 88.

\begin{tabular}{||c|c|c|c|c||}
\hline \hline Survey & Angle $\left(^{\circ}\right)$ & cor-Angle $\left(^{\circ}\right)$ & Vertical-offset $(\mathrm{mm})$ & Specoff $(\mathrm{mm})$ \\
\hline A1379 & 16.5 & 16.489 & 2.31 (upstream) & $0.54(\mathrm{up})$ \\
\hline
\end{tabular}

foils target is used to determine the optics-off. The events from the central foil are selected to get $\mathrm{Y}_{t g}$ information. Using Equation 4.31 and combining information from data and survey, the optics-off is obtained. This offset is used for other single foil targets from different kinematics to calculate the spectrometer offsets.

\section{Pointing results}

Table 4.7 is the summary of the pointing study for every run in the E08014 data set. The pointing study was performed whenever the spectrometer moves from one angle 
to another. The correction to the angle setting is very small based on this study. For the kinematic settings of this experiment, this small correction in the scattering angle can introduce a $1.5 \%$ to $2 \%$ variation in the cross section based on the XEMC cross section model. It is necessary to correct for this offset.

Table 4.7: Summary of Pointing study through every run in XGT2 data

\begin{tabular}{||c|c|c||}
\hline \hline Run Number & Setting Left Angle $\left(^{\circ}\right)$ & Corrected Left Angle $\left(^{\circ}\right)$ \\
\hline $3565-3656$ & 25 & 24.98 \\
\hline $3657-3683$ & 21 & 20.98 \\
\hline $3684-3708$ & 23 & 22.98 \\
\hline $3735-3891$ & 25 & 24.98 \\
\hline $3892-3916$ & - No Left- & 27.98 \\
\hline $3917-4071$ & 28 & 20.99 \\
\hline $4073-4103$ & 21 & 22.98 \\
\hline $4112-4179$ & 23 & 24.97 \\
\hline $4181-4241$ & 25 & 20.99 \\
\hline $4242-4250$ & 21 & 27.98 \\
\hline $4251-4299$ & 28 & Left - \\
\hline
\end{tabular}

\subsubsection{Cross section Model (XEMC)}

The XEMC cross section package is written in $\mathrm{C}++$. The structure of the XEMC package is summarized in Figure 4.49. This cross section model provides both the Born and the radiative cross section. The Born cross section is the sum of Deep Inelastic (DIS) cross section and the quasi-elastic cross section. The global fitting of DIS, encapsulated in routine, F1F209, is used for the DIS cross section and the scaling $F(y)$ function is used for the quasi-elastic cross section. To be able to get the radiated cross section the elastic tail and quasi-elastic tail are calculated. The radiative correction procedure follows that of Ref. [90]. Note that in this cross section model package the energy loss is included and based on the amount of material the 
particle passes through. This radiative cross section is used to weight the output of the SAMC and it is compared to the data to get the yield ratio and then the cross section (Section 5.2). After the first iteration a cross section obtained, and a new fit is performed to improve the cross section model. Only a few iterations are required to get the final cross section model.

Cross section model XEMC package structure

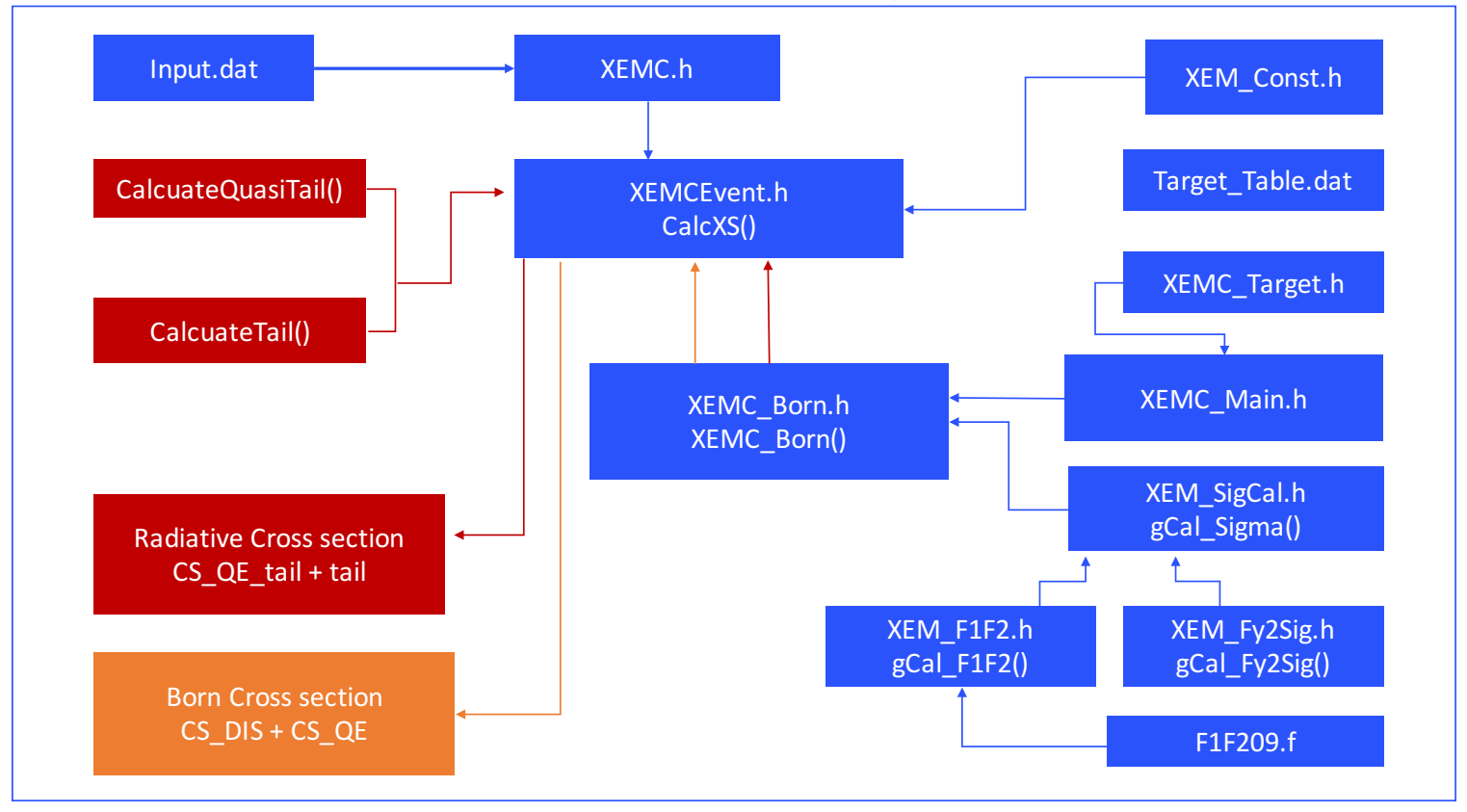

Figure 4.49: The cross section model XEMC package structure

\subsubsection{Data and simulation comparison}

After all offset information was set in the simulation, the initial radiative cross section tables are created, we are ready to compare data and simulation. How the absolute scaling factor work to scale the simulation is discussed detail in Chapter 5. Figure 4.50 shows the comparison over the full acceptance using the initial optics which had a problem in $\theta_{t g}$ reconstruction as mentioned in the Section 4.4. After the optics change to the $\mathrm{G}_{M}^{p}$ optics, the dip in the $\theta_{t g}$ reconstruction was fixed, see Figure 4.51. The offset information from the pointing and all correction are included in the simulation 
to provide the comparison in the Figure 4.52. The data and simulation agree with each other very well over a large range of the acceptance in both momentum $( \pm 3.5 \%)$, in-plane angle $\phi_{t g}( \pm 30 \mathrm{mrad})$ and out-plane angle $\theta_{t g}( \pm 35 \mathrm{mrad})$. This result is a big improvement because we get the good agreement over a much wider range to compare to nominal cuts used in cross section analysis in Hall a. The above cuts are used for the cross section analysis of this data.

\section{Data, simulation comparison using the initial optics}
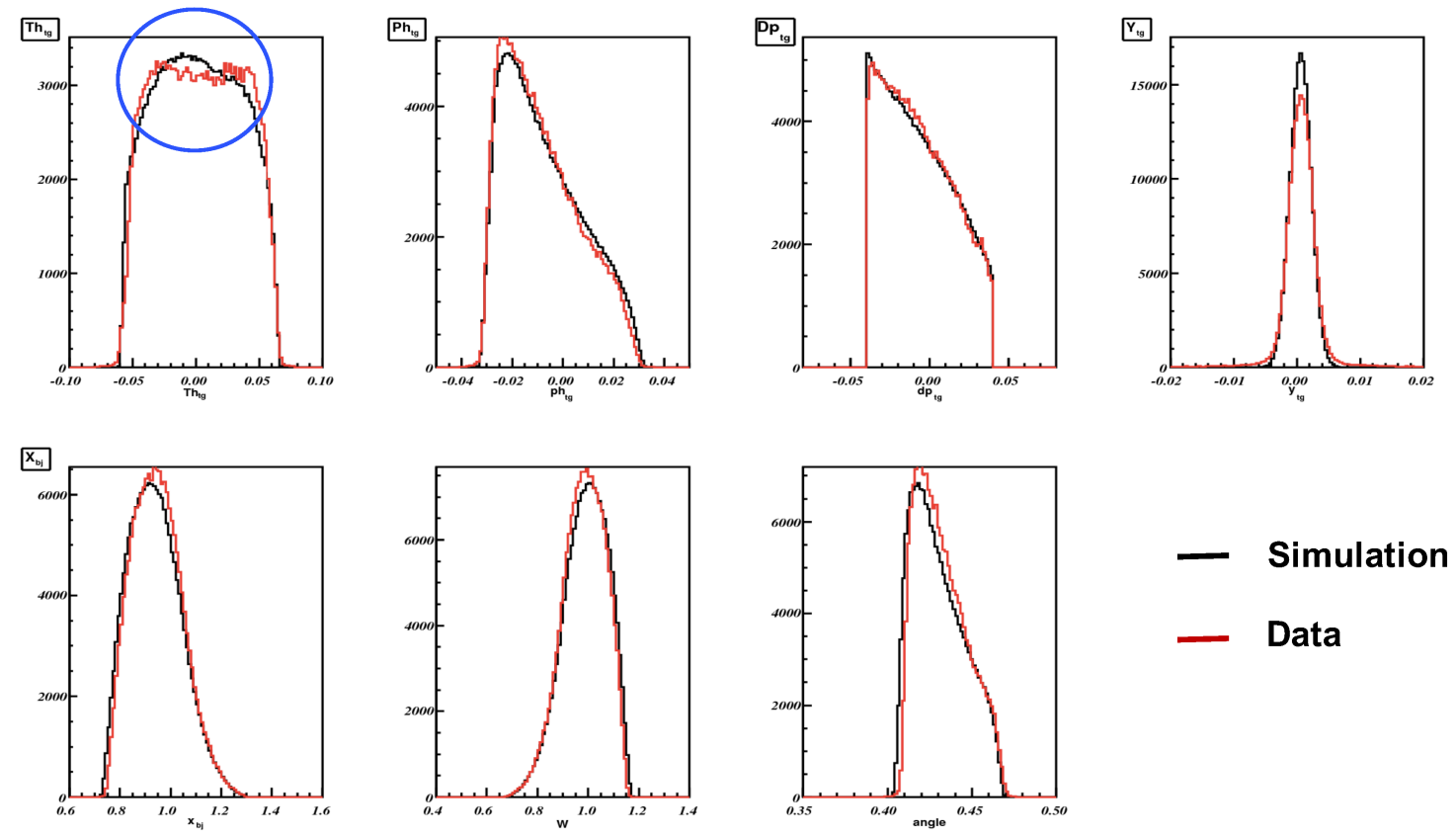

Figure 4.50: Data and simulation comparison using initial optics. 
Data, Simulation comparison using the GMP optics
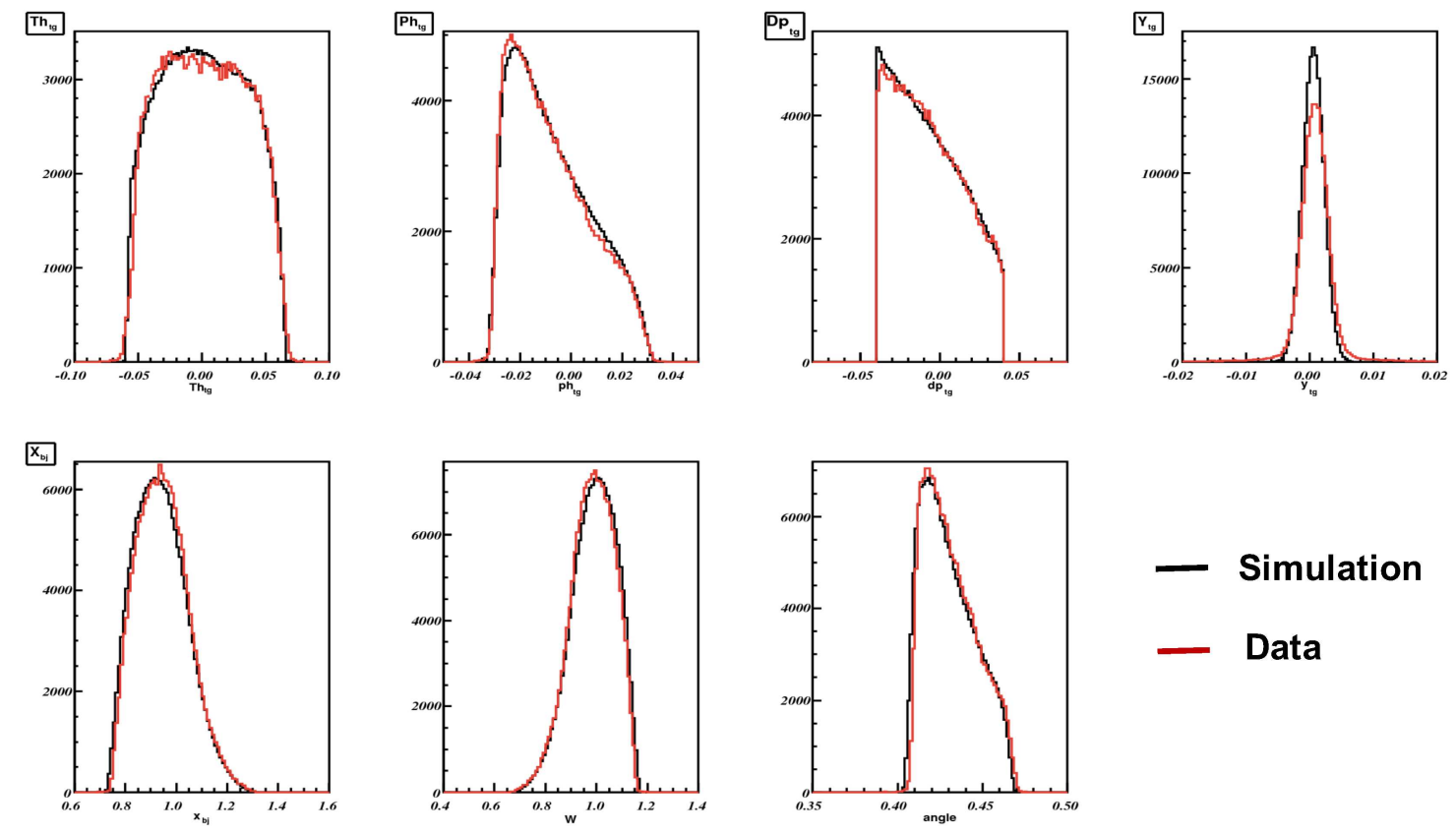

- Simulation

Figure 4.51: Data and simulation comparison using $\mathrm{G}_{M}^{p}$ optics.
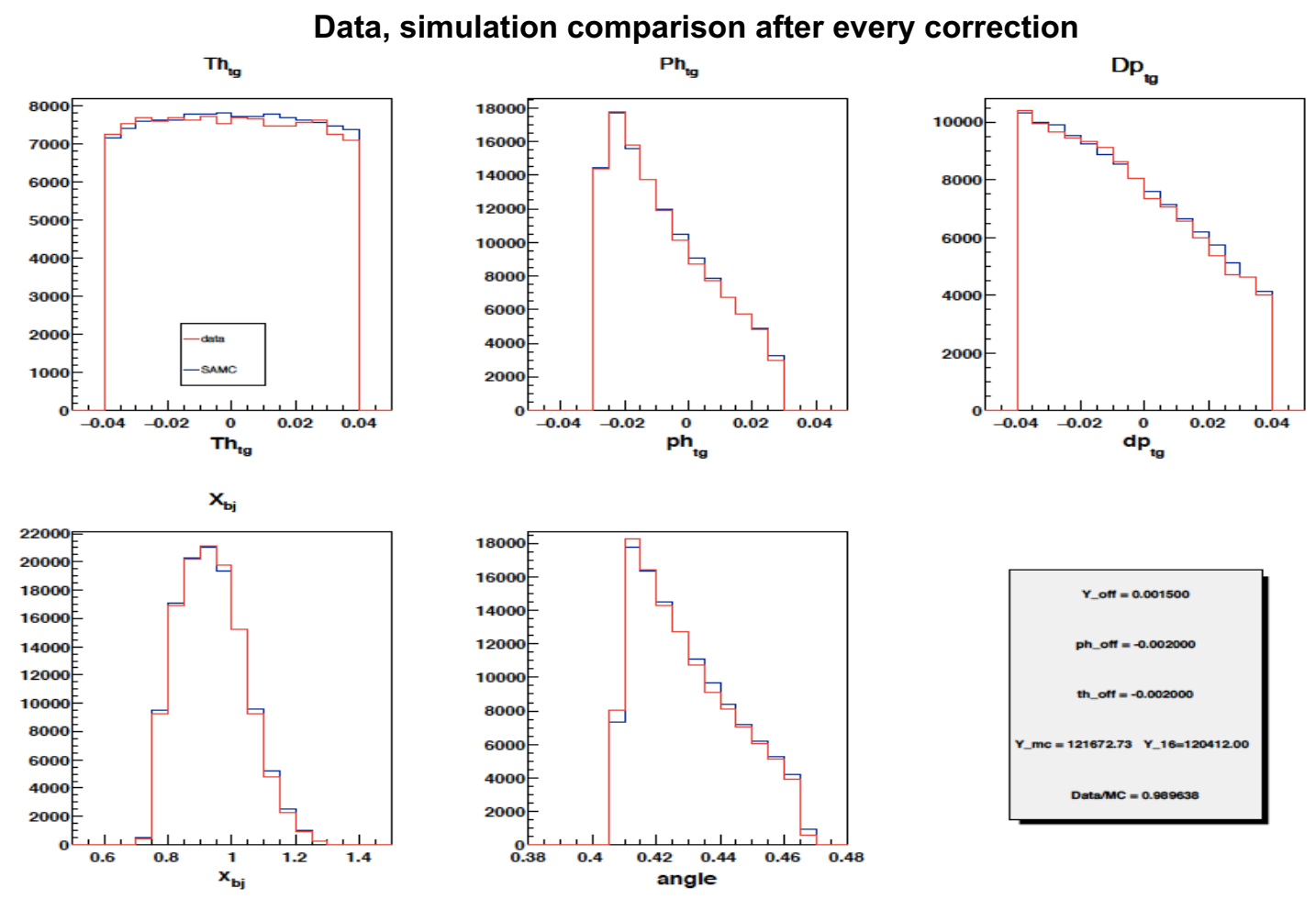

Figure 4.52: Data and simulation comparison after applying every correction. 


\section{Chapter 5}

\section{Cross Section Analysis}

\subsection{Overview}

This section will give an introduction to the procedure and the methods for the extraction of the cross section from the data. Only data from the LHRS is used in this analysis. The approach for inclusive experiments starts with the number of detected electrons, $N$, after selection cuts as discussed in Chapter 4 and summarized here:

- Tracking cut: only select events with a single track (Section 4.5.4).

- Trigger cut: only select events from the trigger of interest.

- PID cuts: select electrons using $(c e r-$ sum $>50)$ and $(E / p>0.5)$ which means that the ADC channel for the sum of the Cherenkov signals must be greater than 50 and the ratio of the deposited energy $E$ in the calorimeter to the momentum $p$ of the particle has to be greater than 0.5 (Section 4.5.3).

- Acceptance cuts: use a range from target variables $\left|d p_{t g}\right|<0.035,\left|\theta_{t g}\right|<0.035,\left|\phi_{t g}\right|<0.025$ and $\left|Y_{t g}\right|$ within $\pm 5 \sigma$ (Section 4.6). 
Here, the number of detected electrons, $N$, is related to the cross section 91,92 through

$$
N=\frac{d \sigma}{d E d \Omega} \cdot L \cdot \Delta E \cdot \Delta \Omega \cdot A_{c o r} \cdot B_{c o r} \cdot \epsilon
$$

where $\frac{d \sigma}{d E d \Omega}$ is the double differential cross section which we want to extract, $\Delta E$ and $\Delta \Omega$ are the momentum and solid angle phase-space, respectively. The terms $A_{\text {cor }}$ and $B_{\text {cor }}$ are the acceptance and binning corrections, respectively, while $\epsilon$ is the total detection efficiency. $L$ is the integrated luminosity, the product of target areal density in terms of nuclei $/ \mathrm{cm}^{2}$ and the total beam charge in terms of the number of electrons;

$$
L=Q_{e} \cdot \frac{\rho_{v} \cdot l}{A} \cdot N_{A}
$$

where $Q_{e}$ is the total beam charge, $\rho_{v}$ and $l$ are the volume density and the thickness of the target, $\mathrm{A}$ is the atomic weight and $N_{A}$ is Avogadro's number. If we know every quantity in the Equation 5.1, the cross section can be obtained. The following sections will present the methods used to extract the cross section.

\subsection{Two Methods to extract the cross section}

The two most common methods of extracting the cross section are 1) the acceptance correction method and 2) the yield ratio method [23, 25, 93]. Note that when we extract the cross section from data we bin it in the kinematic variables, such as $\nu$ or $x_{b j}$. Henceforth the differential cross section for each bin, $\frac{d \sigma}{d E d \Omega}$, will be referred to as $\sigma\left(\chi_{0}^{i}, \theta_{0}\right)$, where $\chi$ can be any kinematic variable we use to bin data, $\theta_{0}$ is the scattering angle for each setting and $\chi_{0}^{i}$ is the center of bin $\chi^{i}$. 


\section{Acceptance correction method}

This method uses the efficiency corrected Yield ${ }^{d a t a}\left(\chi^{i}, \theta_{0}\right)$ for each bin $i$, defined as the total number of detected electrons for that bin corrected by the detection efficiency.

$$
\text { Yield }^{\text {data }}\left(\chi^{i}, \theta_{0}\right)=\frac{N^{i}}{\epsilon}=\sigma^{\text {data }}\left(\chi_{0}^{i}, \theta_{0}\right) \cdot L^{\text {data }} \cdot \Delta E \cdot \Delta \Omega \cdot A_{c o r} \cdot B_{c o r},
$$

The $\Delta E, \Delta \Omega, A_{\text {cor }}, B_{\text {cor }}$ are calculated for each bin $\chi^{i}$. This method assumes all corrections can be calculated and applied separately to get the cross section. The most difficult part is getting the correction factors $A_{c o r}$ and $B_{c o r}$, the acceptance and bin centering corrections. Incorporating radiative correction is also challenging in this method.

- Acceptance correction: The acceptance was defined in the Section 4.6. It is the probability that a particle will pass successfully through the spectrometer. This probability depends on the vertex coordinates defined by $(x, y, z)$ and the momentum vector defined by $\left(E, \theta_{t g}, \phi_{t g}\right)$ of the particle at the target. So the acceptance correction then can be written as a function $A\left(x, y, z, E, \theta_{t g}, \phi_{t g}\right)$. The cross section is independent of $(x, y, z)$ if the target is thin enough so the loss of beam intensity can be ignored so that it is just a function of $E, \theta_{t g}$ and $\phi_{t g}$. Since $\theta$ is a function of $\theta_{t g}$ and $\phi_{t g}$, the acceptance can be properly averaged over $(x, y, z)$ and is a function $A(E, \theta)[23]$. A simulation is used to determine this correction by checking whether the trajectory of each event either passes through the spectrometer or is lost. The acceptance correction can be calculated bin by bin by taking the ratio of the number events which passed all apertures and recorded $N_{r e c}^{i}(E, \theta)$ to the total generated $N_{g e n}^{i}(E, \theta)$ for that bin,

$$
A\left(E^{i}, \theta^{i}\right)=\frac{N_{r e c}^{i}\left(E^{i}, \theta^{i}\right)}{N_{g e n}^{i}\left(E^{i}, \theta^{i}\right)} .
$$


- Binning correction: The cross section is to be quoted at the center of the bin $\chi_{0}^{i}$ and the central angle $\theta_{0}$. If the cross section is the linear function of the kinematic variable $\chi$ and scattering angle $\theta$ then there is no bin centering correction. This never is the case. The binning correction is defined as ratio of the cross section at the central of the bin to the average cross section in the same bin.

$$
B\left(\chi_{0}^{i}, \theta_{0}^{i}\right)=\frac{\sigma\left(\chi_{0}^{i}, \theta_{0}\right)}{\left\langle\sigma\left(\chi^{i}, \theta^{i}\right)\right\rangle}=\frac{\sigma^{M C}\left(\chi_{0}^{i}, \theta_{0}\right)}{\sum_{N_{r e c}^{i}} \sigma^{M C}\left(\chi^{i}, \theta^{i}\right) / N_{r e c}^{i}\left(\chi^{i}, \theta^{i}\right)}
$$

\section{Yield ratio method}

For this method we need to apply the same correction factors as above but in a different way. The effects appearing in data such as the acceptance correction, are applied in simulation to generate pseudo data for the different kinematic settings and are weighted by radiative cross section from the model, as discussed in Section 4.6 . The yield from the simulation, Yield ${ }^{M C}\left(\chi^{i}, \theta_{0}\right)$, using the same cuts and binning as data can be written as:

$$
\operatorname{Yield}^{M C}\left(\chi^{i}, \theta_{0}\right)=\sigma^{M C}\left(\chi_{0}^{i}, \theta_{0}\right) \cdot L^{M C} \cdot \Delta E \cdot \Delta \Omega \cdot A_{c o r} \cdot B_{c o r},
$$

where $\sigma^{M C}\left(\chi_{0}^{i}, \theta_{0}\right)$ is the cross section from the model. The luminosity $L^{M C}$ is set to the same as value as the measured data. If the experimental effects are properly

simulated, the yield ratio (Yield ${ }^{d a t a} /$ Yield $^{M C}$ ) should be very close to 1 . The yield ratio is the only correction factor in the cross section calculation. The experimental cross section is the product of the yield ratio and the cross section from the model,

$$
\sigma^{\text {data }}\left(\chi_{0}^{i}, \theta_{0}\right)=\frac{\text { Yield }^{\text {data }}\left(\chi^{i}, \theta_{0}\right)}{\operatorname{Yield}^{M C}\left(\chi^{i}, \theta_{0}\right)} \cdot \sigma^{M C}\left(\chi_{0}^{i}, \theta_{0}\right)
$$

These two methods were used in analyses of a previous experiment 25] and the 
cross sections obtained using both methods were found to be in good agreement. While the acceptance correction method assumes that all the correction factors factorize and can be calculated and applied separately, the yield ratio method considers the acceptance and binning corrections together which automatically includes the correlations between the corrections and reduces the systematic uncertainty. The yield ratio method also assumes the radiation and resolution effects are described correctly in the simulation. The bias caused by the choice of different cross-section models and simulation tools is also reduced in the yield ratio method. In addition, the yield ratio approach allows for easy checks of the spectrometer acceptance and data quality. In this analysis, we used the yield ratio method to extract the cross section from the comparison between data and the simulated spectra. The details are discussed in the next section.

\subsection{Yield ratio for overlap kinematics}

Overlap kinematics are the kinematics which have the same scattering angle and an overlap in momentum. The utility of overlap kinematic settings is that the entire procedure can be tested before we extract the cross section. As mentioned above the yield ratio method is that if everything is handled correctly the yield ratio should be very close to one. The primary factors that cause the yield ratio to deviate from one are the optics, the acceptance and the cross section model. In the overlap region, the optics and the cross section are the same and the acceptance is nearly identical (98\% agreement over the acceptance range) as presented Section 4.6. The expectation is that the yield ratio will agree very well in the overlap region. A check was performed

on the three overlap kinematics (kin5.0, kin5.05, kin5.1) using the ${ }^{12} \mathrm{C}$ target. The yield ratio binned in both $\mathrm{E}$ and $\mathrm{x}_{b j}$ is shown in Figures 5.1 and 5.2 . The agreement in the overlap region was not as expected and when binned in E' the difference was 


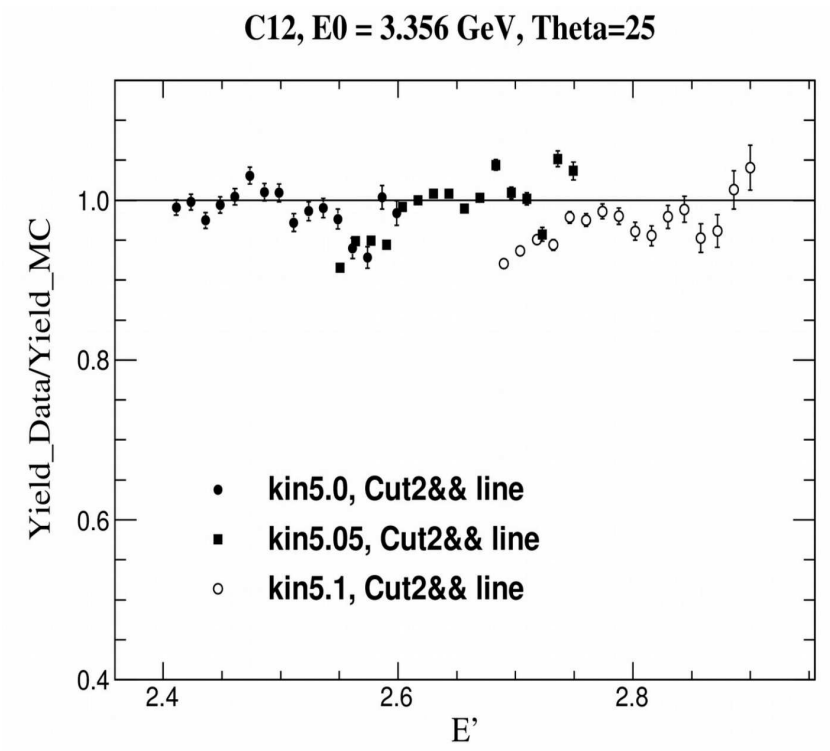

Figure 5.1: Data/MC Yield ratio for 3 different overlap kinematics binned in Ep

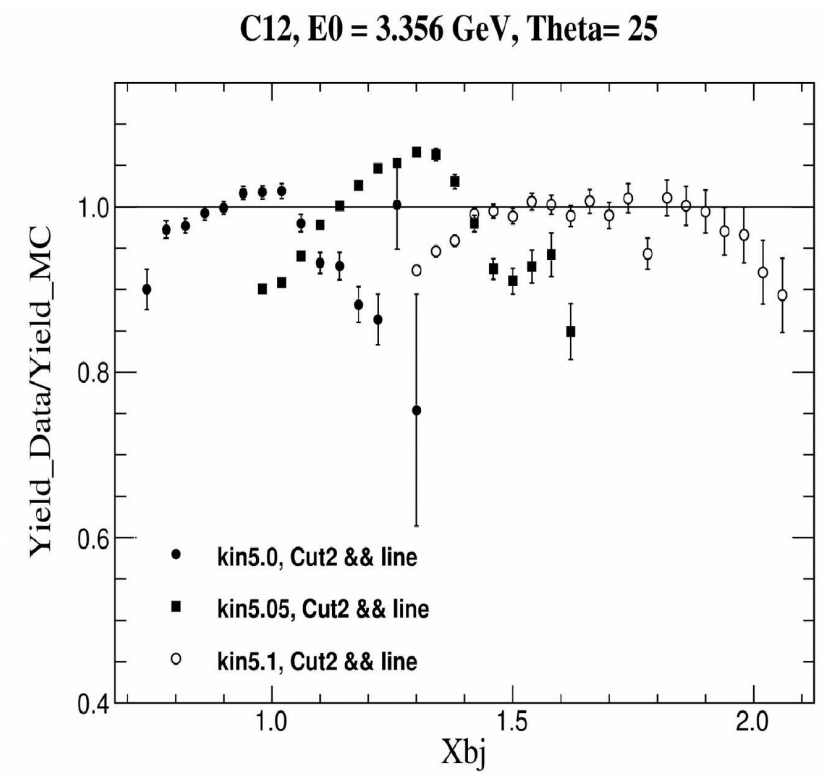

Figure 5.2: The Yield ratio for 3 different overlap kinematic settings binned in $\mathrm{x}_{b j}$. The yield ratio in the overlap region was not in good agreement.

found to be $10 \%$. When binned in $x_{b j}$ the disagreement was worse, as large as $15 \%$. This suggested that there were still some problems to be investigated and taken into account. The drop in the yield ratio in $\mathrm{E}^{\prime}$ bins happens at high $\mathrm{E}^{\prime}$ for every kinematic setting and almost at the same value of the relative momentum $\mathrm{dp} \sim 3 \%$. 
This suggested there may a local inefficiency yet undiscovered. The following section will discuss about how the issue was located and resolved.

\subsection{Local efficiency issue}

As just mentioned the yield ratio of overlap kinematics indicated a low local inefficiency in the data. All the inputs to the yield ratio are reasonable and there is good agreement of the acceptance when data and simulation are compared for all target variables in 1-dimension. But the acceptance is complicated and is multiple dimensional, so a local efficiency is very difficult to detect in a 1 dimensional acceptance comparison. It is natural to check the yield ratio in higher dimensions to locate the problem.

\section{Yield ratio in higher dimension}

Following the same method of calculation used for the yield ratio we now calculate it in two dimensions of the target variables $\left(\theta_{t g}: \phi_{t g}\right),\left(\phi_{t g}: d p_{t g}\right)$ and $\left(\theta_{t g}: d p_{t g}\right)$. Figures 5.3 and 5.4 show the yield ratio in a 2-dimensional phase space of the target variables. Figure 5.3 shows that the yield ratio is uniform all over the two-dimensional phase space acceptance except for a few points at the edge where the acceptance is not well described. Figure 5.4 shows an obvious low yield ratio in a specific region, $\mathrm{dp}=3 \%$ and $-0.005<\phi_{t g}<0.03$, which indicates a local efficiency problem.

\section{Local efficiency at Trigger level}

The local efficiency was confirmed and it could come from various sources: detectors, tracking or trigger efficiency. After checking through all possible sources the inefficiency was isolated to the trigger. The trigger design of this experiment was discussed in the Section 3.6.5. The main trigger (T3) used in the analysis is $\left(S_{0} \& S_{2} \&\right.$ 


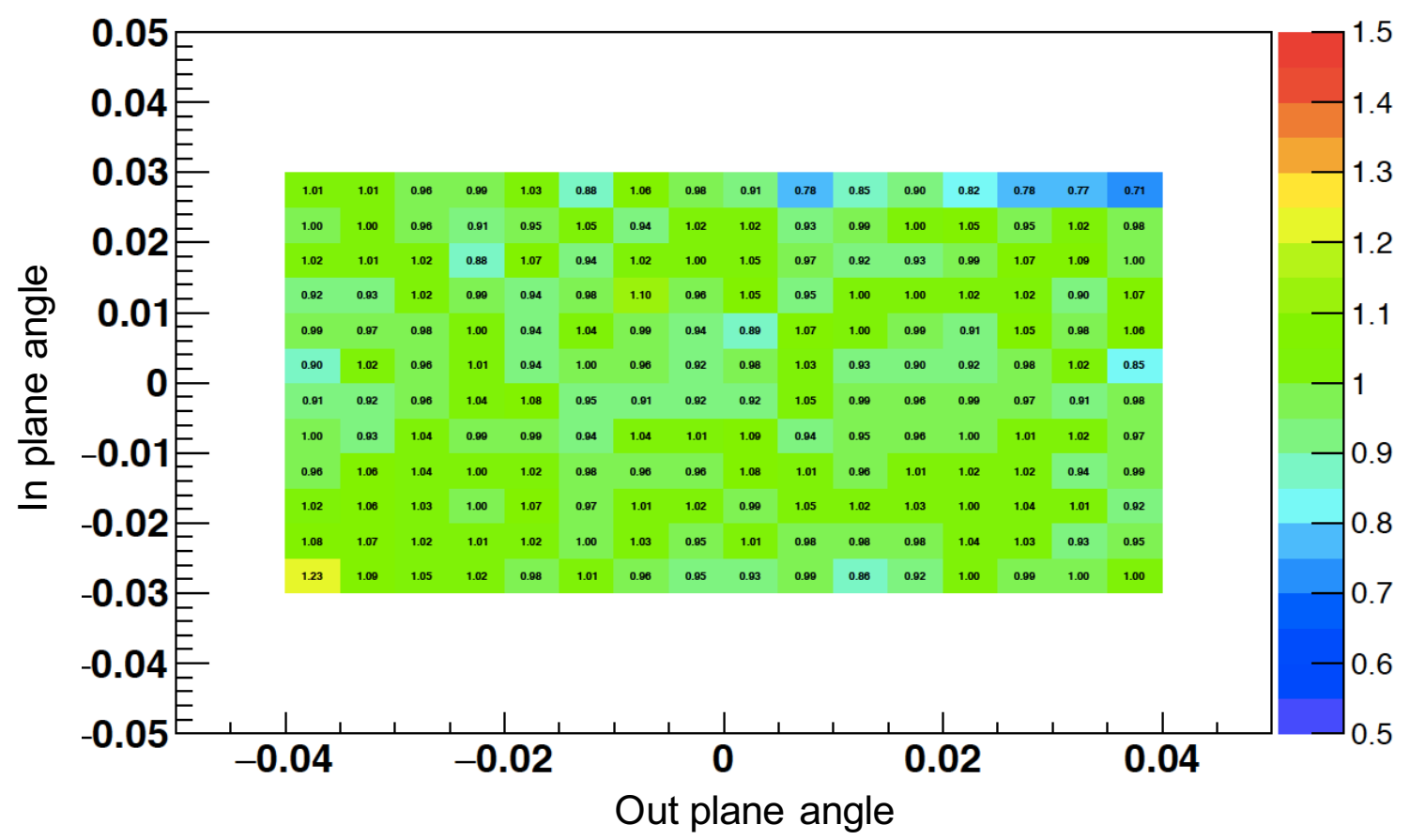

Figure 5.3: 2D Yield ratio as function of in plane angle $\phi_{t g}(\mathrm{rad})$ vs out plane angle $\theta_{t g}(\mathrm{rad})$ shows a uniform distribution except few points at the edge where acceptance was not modeled well.

Cer) while the efficiency trigger (T7) is the combination $\left(S_{0} \& S_{2}\right)$. The event distribution in phase space of Cherenkov detector $\left(\operatorname{cer}_{x}\right.$ vs $\left.\operatorname{cer}_{y}\right)$ corresponds to two type of event triggers, T3 and T7, are shown in the Figures 5.5 and 5.6, respectively. The T3 event distribution shows a dip around $\operatorname{cer}_{x}=0.4$ (where it is normal for the T7 event distribution) and the projection of the events in the low yield ratio area seen in the Figure 5.4 onto the phase space of Cherenkov appear at the same location of the dip seen in Figure 5.5. Figure 5.7 shows the locations of 10 PMTs in the Cherenkov. The location of the low efficiency region is at the position of PMT7. The next steps describe how to calculate and correct this local inefficiency in the yield ratio. 


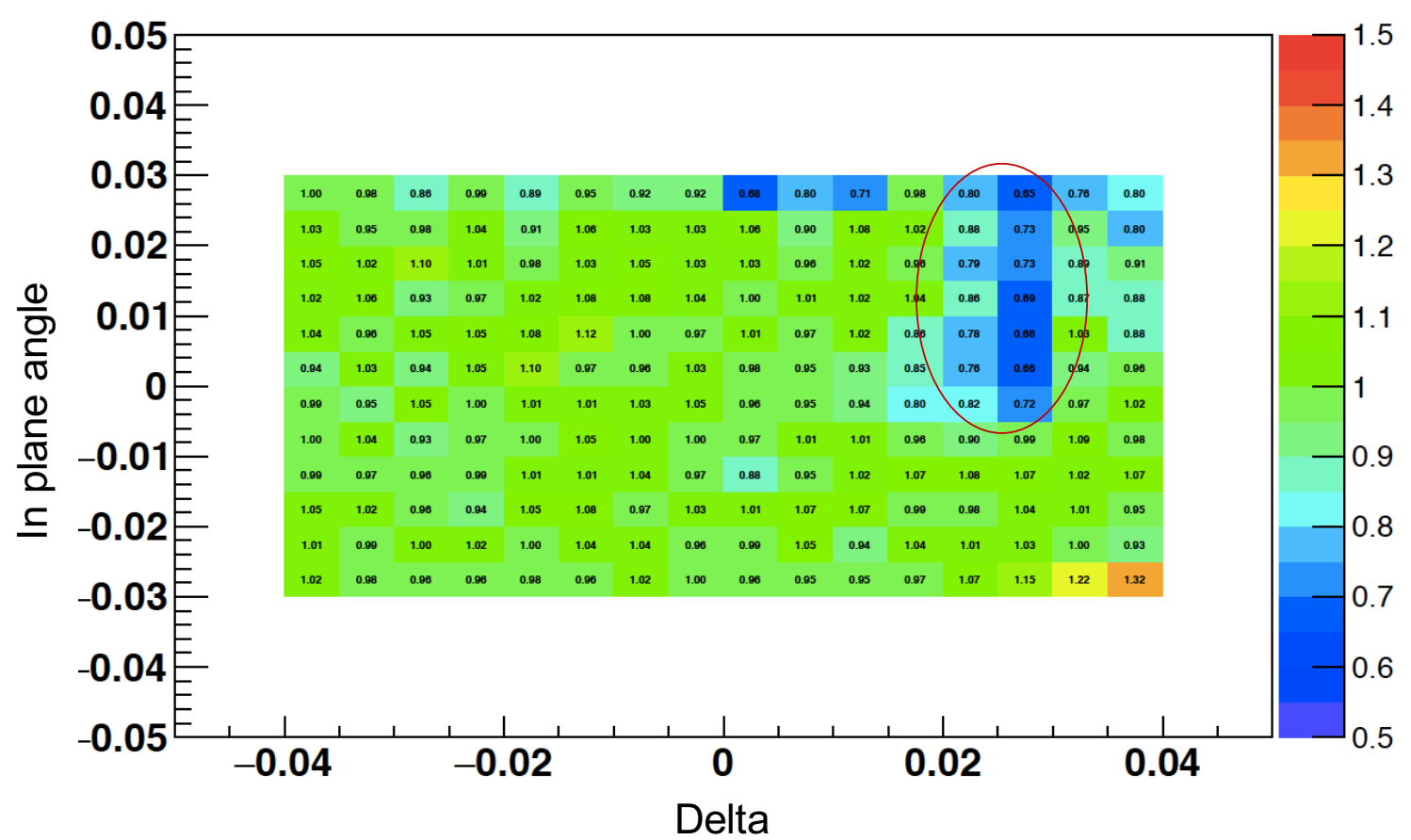

Figure 5.4: The 2D Yield ratio as function of in plane angle $\phi_{t g}$ (rad) vs relative momentum dp shows a specific region where the yield ratio drops. This occurs at $\mathrm{dp} \sim 0.03$ and $\phi_{t g}>-0.005$.

Trigger 3 events distribution on the Cherenkov phase space

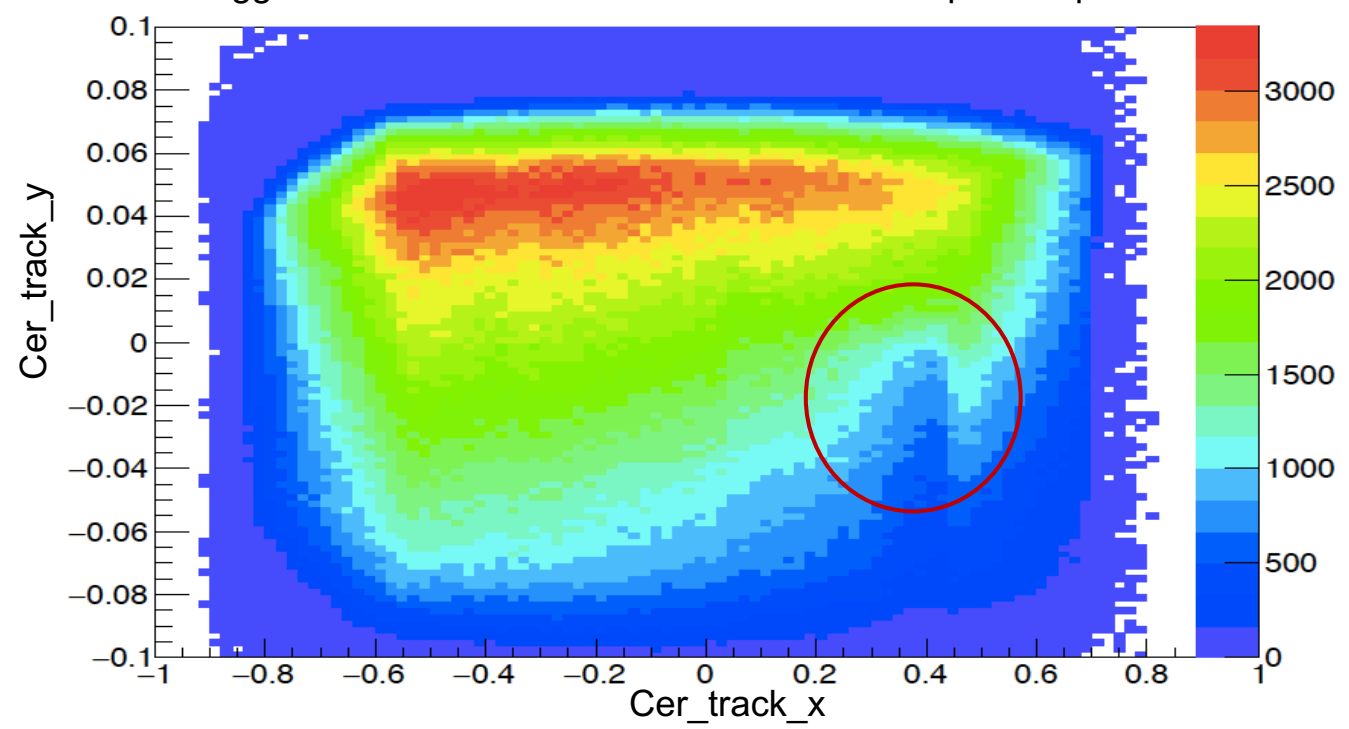

Figure 5.5: The Trigger 3 event distribution on the Cherenkov phase space. The dip is obvious at the location within the red circle. 


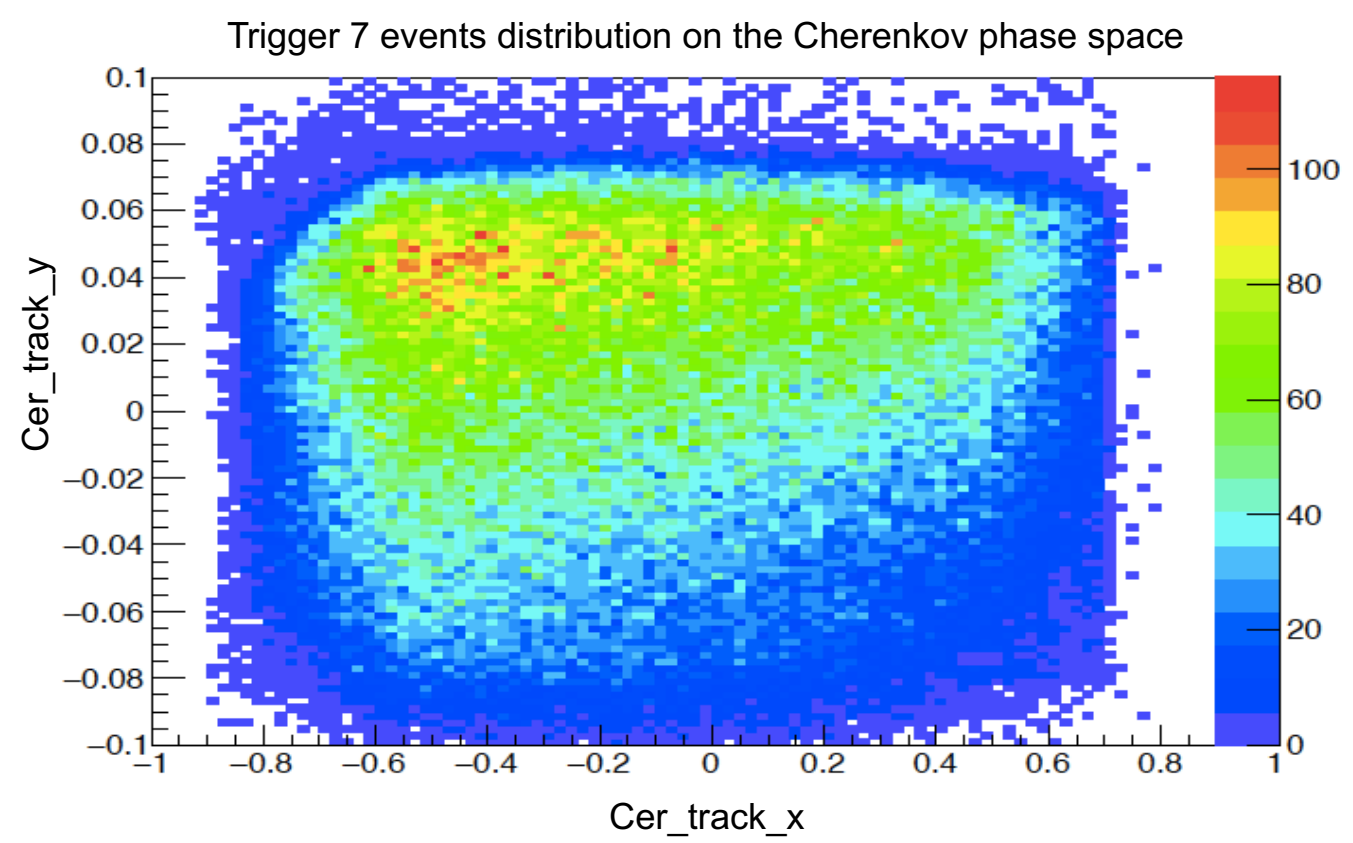

Figure 5.6: The trigger 7 events distribution for the same data as in Figure 5.5. It is clear that the dip region doesn't exist in the distribution when we switch from Trigger 3 to Trigger 7

PMTs location in Cherenkov phase space

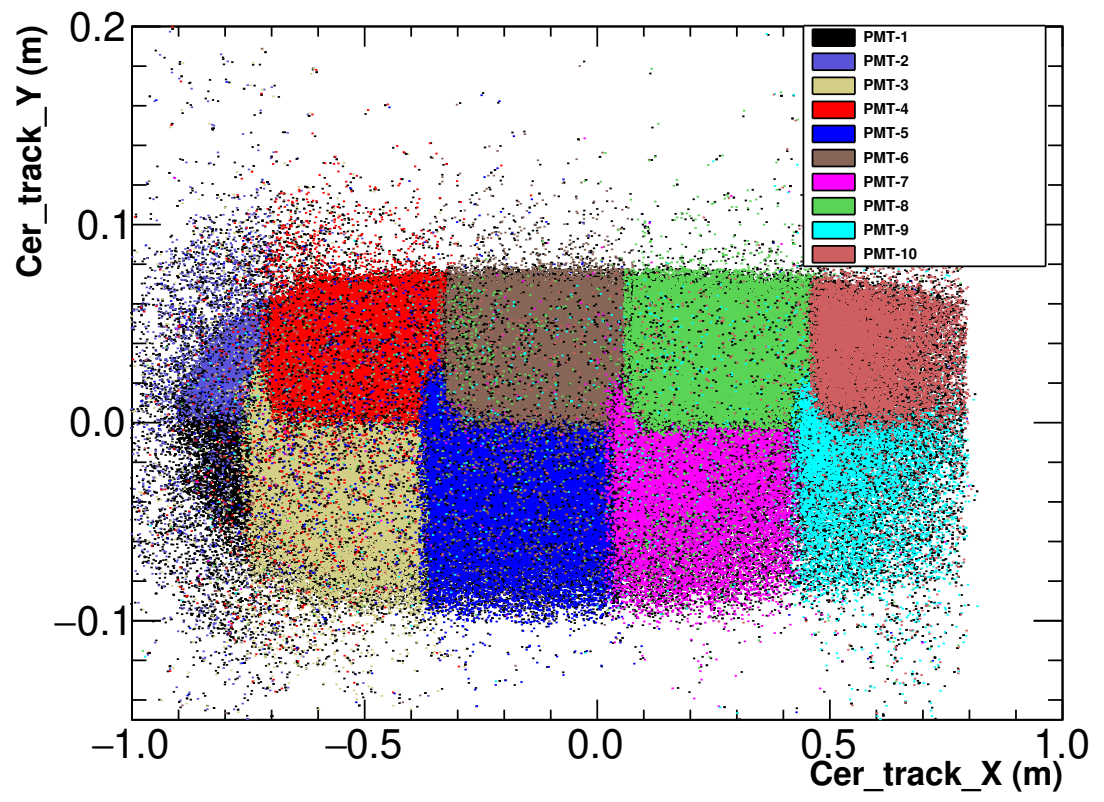

Figure 5.7: The location of each of the 10 PMTs in the Cherenkov phase space. 


\section{Local efficiency calculation}

There are two ways to calculate the local efficiency correction. The first is to calculate the efficiency as a function of the Cherenkov position $\left(\operatorname{cer}_{x}: \operatorname{cer}_{y}\right)$ as seen in Figure 5.8 . Then, this map can be used to apply the correction event by event depending on the track position in Cherenkov phase space.

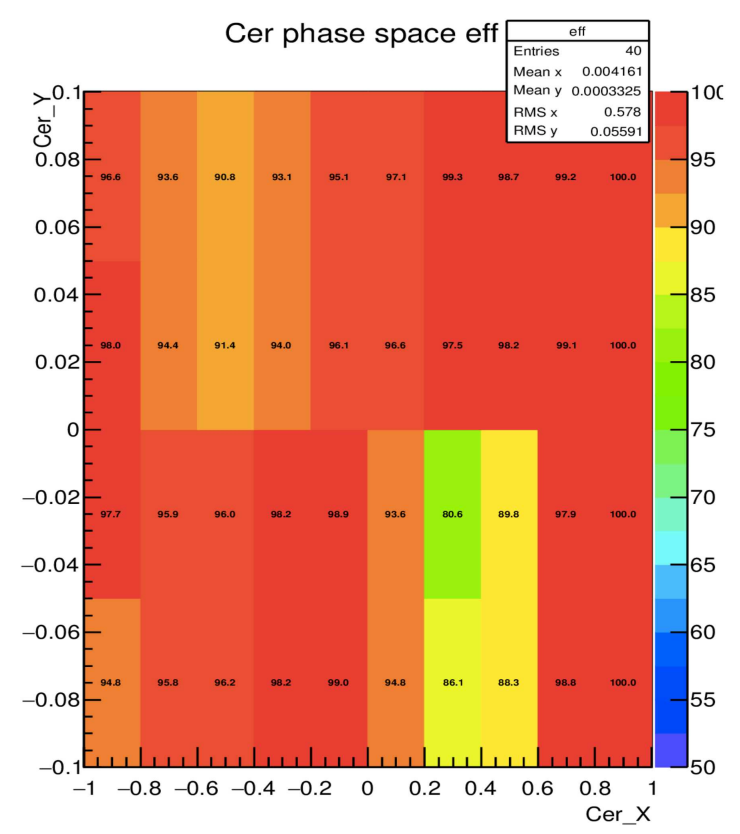

Figure 5.8: The plot shows the local efficiency as function of Cherenkov position.

The second way is to calculate the efficiency as the function of $x_{b j}$ or $E^{\prime}$ with the same binning as we have for the yield ratio, then apply the correction to the yield ratio bin by bin. See Figure 5.9. The difficulty in calculating of the local trigger efficiency comes from the fact that when the data taking was set up a high pre-scale factor was applied to efficiency Trigger T7. This means that the statistics for the efficiency calculation are low. This issue can be worked around by summing all the data in the same kinematic setting for each target together. However a bigger problem was that pre-scale factor for T7 was changed multiple times throughout the data taking period. With the different pre-scale factor on $\mathrm{T} 7$ we need to be very careful when 

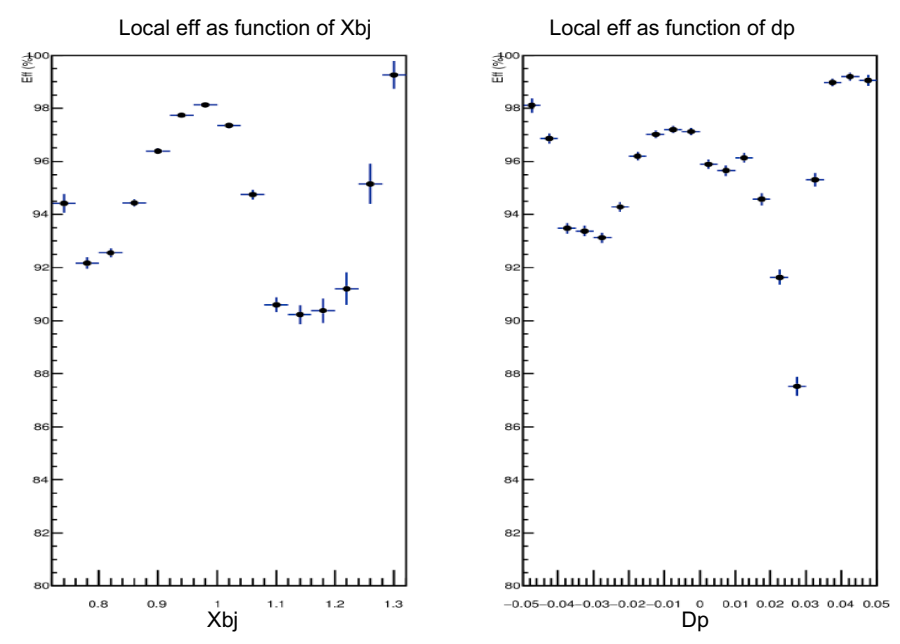

Figure 5.9: The left plot is the local efficiency as function of $x_{b j}$ for one kinematics setting. The right plot is the local efficiency as function of $\mathrm{d}_{p}$ for the same setting as in the left plot.

combining runs together. The first step was to set loose cuts on the acceptance, on the tracking and on the PID using the E/p to select events. The second step was to select the sample, N7, of events which pass the selection cuts and fire T7. The sample N73 includes the events in the sample N7 which also fires T3. The efficiency can be determined as

$$
\mathrm{Eff}=\frac{N 73}{N 7}
$$

With this selection sample, N7, we don't need to worry about about the difference in pre-scale factor of trigger $\mathrm{T} 7$ but this calculation only works if the Trigger T3 has pre-scale factor of 1 . The uncertainty accompanying our calculation must also be determined. This uncertainty follows from the binomial distribution 87, 91] because the events in sample N7 either fire T3 or not. The approximation 87] for the uncertainty can be calculated using:

$$
\text { Uncertainty }=\text { Eff } * \frac{\operatorname{sqrt}(N 73 *(1-N 73 / N 7))}{N 7} .
$$

This local efficiency depends on the kinematic setting and the target length. Fig- 
ure 5.10 shows the local efficiency as function of dp for every kinematic setting for ${ }^{12} \mathrm{C}$. The unique map to apply this correction needs to be averaged over all different kinematic settings and over different target lengths. The calculation has many different steps and are not presented here but can found in Ref. 94].

\section{C12, Cer_dp correction for all kin setting}

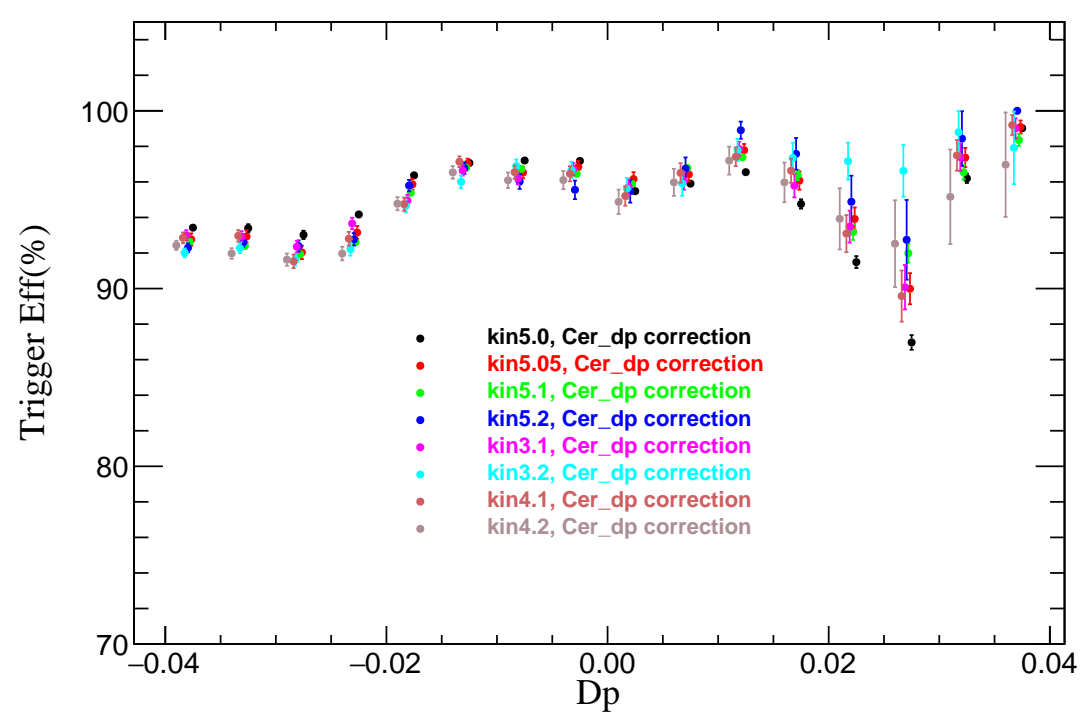

Figure 5.10: The local efficiency as a function of $\mathrm{d}_{p}$ for every kinematic setting for Target ${ }^{12} \mathrm{C}$. The local efficiency varies over the different kinematics.

\section{Improvement in yield ratio}

After the local efficiency was calculated bin by bin in both $\mathrm{E}^{\prime}$ and $x_{b j}$ for each kinematic setting, it can be used as a correction factor to the yield ratio. This results in a significant improvement in the yield ratio for overlapping kinematic settings. The yield ratio in the overlap region now have very good agreement, within a few percent

over different kinematic settings in $\mathrm{E}^{\prime}$ and around $5 \%$ in $\mathrm{x}_{b j}$. See Figure 5.11 and 5.12 .

Both approaches for applying the local efficiency correction in the yield ratio were studied and both returned good agreement. See Ref. 95. 
C12, Yield ratio dp, W/0 correction VS cer_dp

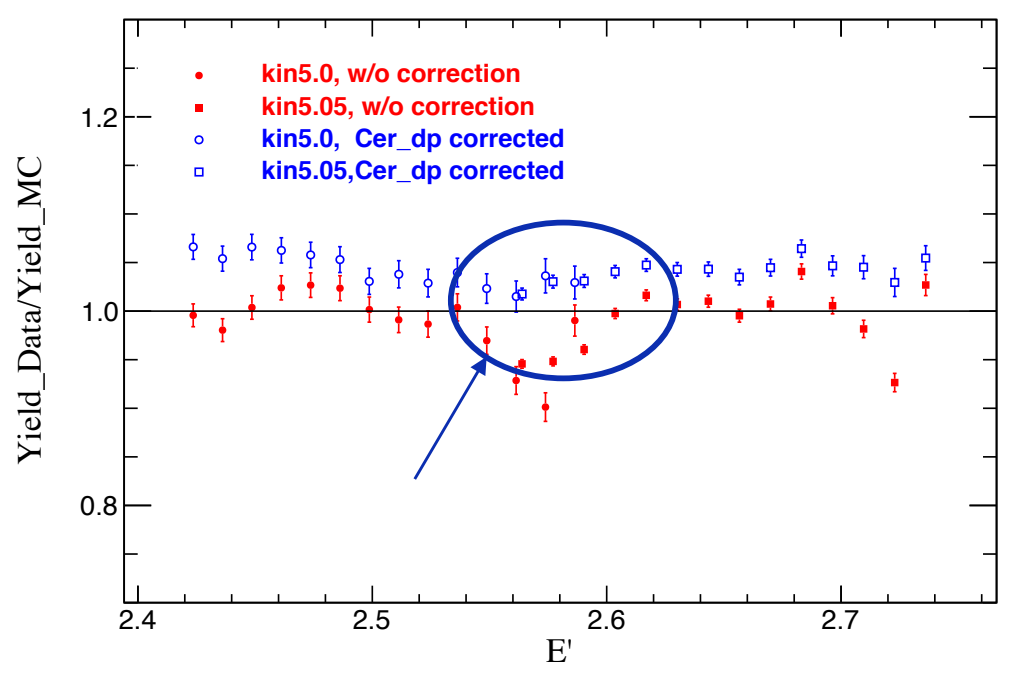

Figure 5.11: The red points are the yield ratio binned in $\mathrm{E}^{\prime}$ for two overlap kinematics settings, Kin5.0 and Kin5.05, before the local efficiency correction was applied while the blue points are the result after the correction. The overlap region shows a significant improvement after application of the correction.

C12, Yield ratio xbj, w/o correction VS cer_xbj

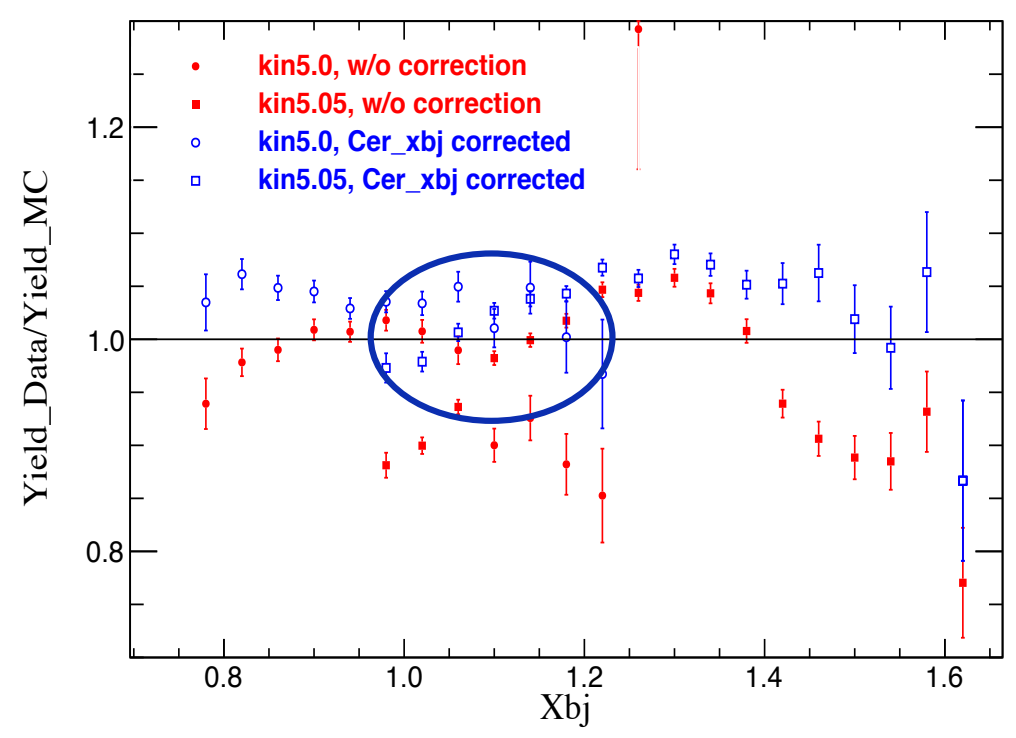

Figure 5.12: The red points are the yield ratio as function of $\mathrm{x}_{b j}$ for two overlap kinematic settings, Kin5.0 and Kin5.05, before the local efficiency correction, and the blue points are the results after the correction. 


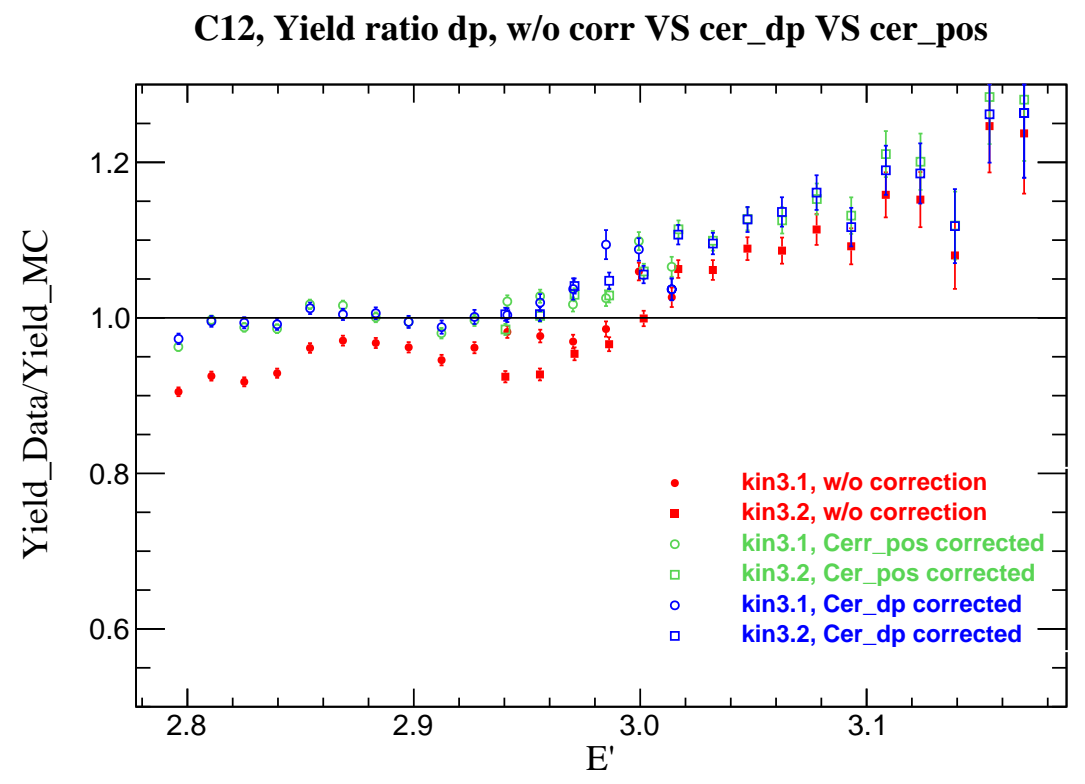

Figure 5.13: The Red point are the yield ratio before local efficiency was applied. The blue points are the yield ratio after local efficiency correction was applied bin by bin in dp. The green points are the yield ratio after local efficiency was applied using the unique map of Cherenkov positions.

In the end, the approache of applying efficiency to the yield ratio bin by bin in $\mathrm{dp}$ and in $x_{b j}$ binning was used in analysis since it is easier to propagate the uncertainty in the yield ratio.

\subsection{Acceptance dependence}

After applying all corrections, now we have very good results for the yield ratio for every kinematic setting. This section will discuss the stability of the yield ratio as acceptance cuts are changed. To be able to conduct this study we need to have multiple set of acceptance cuts as listed in Table 5.1 [96]. A more detailed study of this study was presented in the Ref. 97.

The acceptance Cut1 plays the role of a reference cut. In each other cut, the range in one of target variables is changed systematically. The yield ratio bin-tobin for both $\mathrm{E}^{\prime}$ and $x_{b j}$ for each set of the acceptance cuts are calculated. The 
Table 5.1: The sets of acceptance cuts on the target reconstructed variables

\begin{tabular}{||c|c|c|c|c||}
\hline \hline Cut-name & $\operatorname{dp}(\%)$ & $\theta_{t g}(\mathrm{mrad})$ & $\phi_{t g}(\mathrm{mrad})$ & $\mathrm{y}_{t g}(\sigma)$ \\
\hline Cut1 & 3.5 & 40 & 25 & 5 \\
\hline Cut2 & 3 & 40 & 25 & 5 \\
\hline Cut3 & 4 & 40 & 25 & 5 \\
\hline Cut4 & 3.5 & 35 & 25 & 5 \\
\hline Cut5 & 3.5 & 40 & 20 & 5 \\
\hline
\end{tabular}

difference in the yield ratio from the Cut2-Cut5 to the reference cut is a measure of the acceptance dependence (see Figures 5.14, 5.15, 5.16, 5.17). The variation in the yield ratio using different cuts compared to the yield ratio using the reference cut is very stable. For the most part, the scatter is less than $2 \%$ in bin $x_{b j}$ except for certain cuts at the very edge of the acceptance. For the final results, the $x_{b j}$ range is limited to stay away from the edge points where the acceptance is not modeled well. In addition, some of the cuts in the acceptance dependence studies dramatically change the statistics at the edge points. It means that the variation may come from statistics and data, not because of the acceptance. The bottom line is that we don't need to worry about the large variations at the edges. So for the absolute cross section, a $2 \%$ normalization uncertainty and $1.5 \%$ point-to-point are very conservative for the acceptance dependence. For the cross section ratio of different solid targets, the acceptance effects are canceled almost exactly. The normalization uncertainty is negligible and point-to-point uncertainty should cancel as well. A $0.2 \%$ point-to-point uncertainty for cross section ratio to account for the different offsets between 2 targets position. 

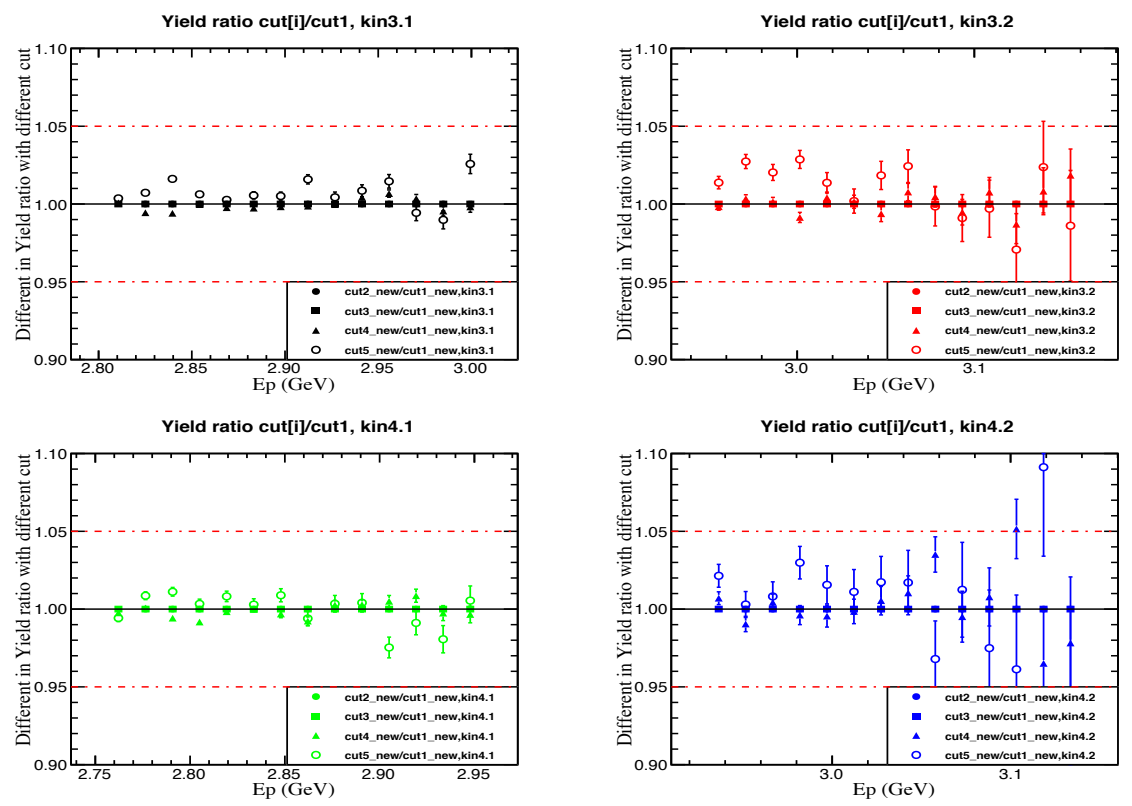

Figure 5.14: The acceptance dependence as a function of $\mathrm{E}^{\prime}$ for $\theta_{0}=21^{\circ}$ and $\theta_{0}=23^{\circ}$. The variations of the yield ratio binned in $\mathrm{E}^{\prime}$ using different cuts (Cut2 - Cut5) to the yield ratio using the reference cut are very small for different kinematic settings. Two red dashed lines show $\pm 5 \%$ variation from one.
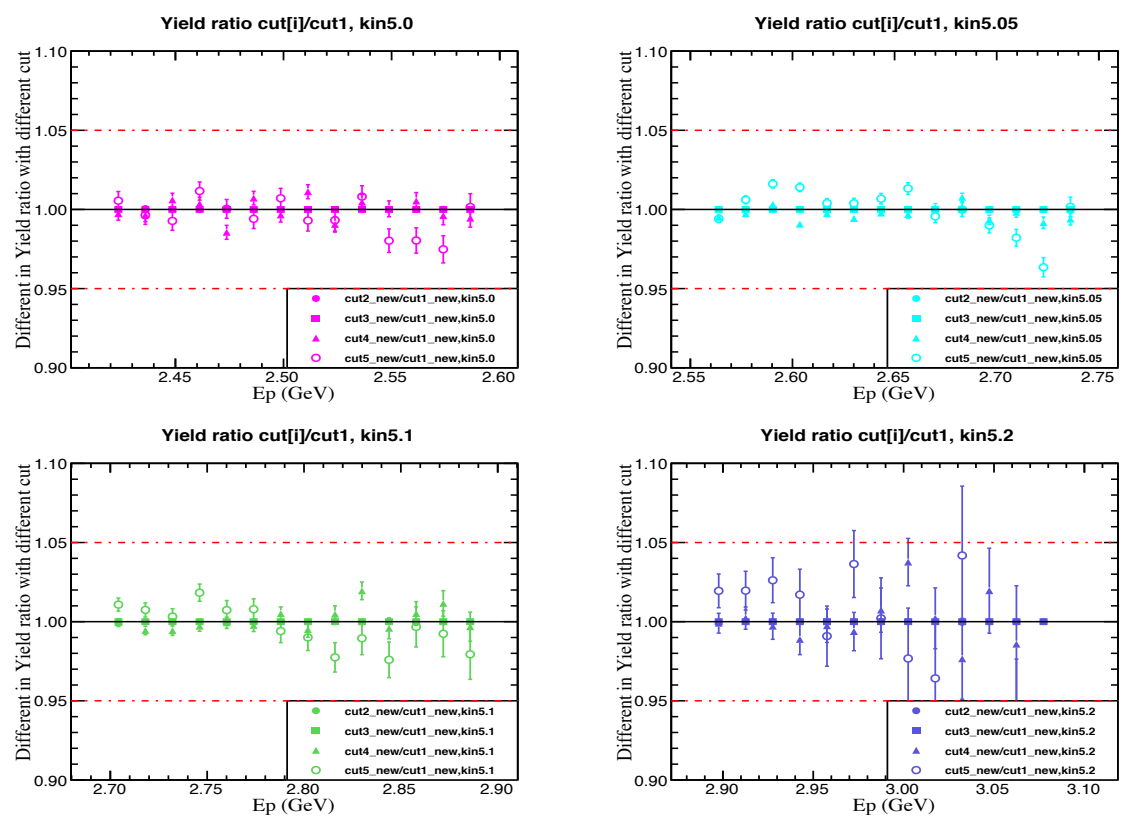

Figure 5.15: The acceptance dependence as a function of $\mathrm{E}^{\prime}$ for $\theta_{0}=25^{\circ}$ settings. The variations of the yield ratio binned in $\mathrm{E}^{\prime}$ using different cuts (Cut2 - Cut5) to the yield ratio using the reference cut are very small for different kinematic settings. Two red dashed lines shows $\pm 5 \%$ variation from one. 

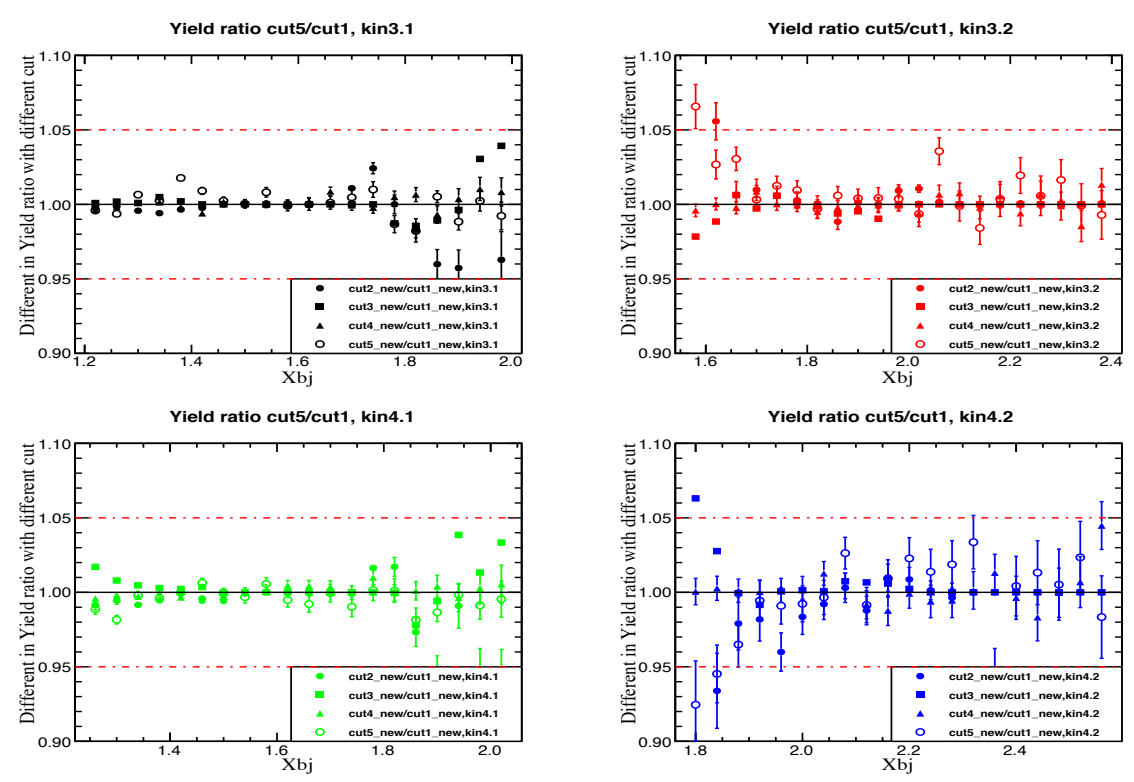

Figure 5.16: The acceptance dependence as a function of $x_{b j}$ for $\theta_{0}=21^{\circ}$ and $\theta_{0}=23^{\circ}$. The variations of the yield ratio binned in $x_{b j}$ using different cuts (Cut2 - Cut5) to the yield ratio using the reference cut are very small for different kinematic settings. Two red dashed lines show $\pm 5 \%$ variation from one.
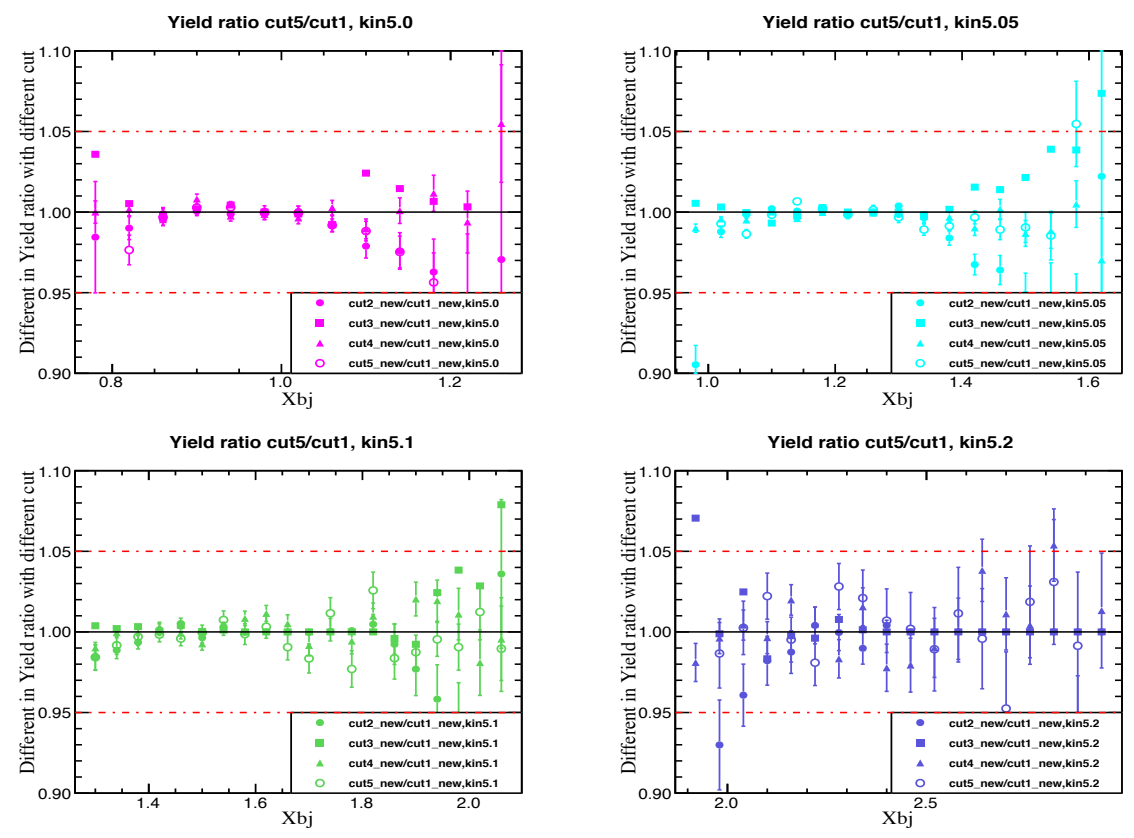

Figure 5.17: The acceptance dependence as a function of $x_{b j}$ for $\theta_{0}=25^{\circ}$ settings. The variation of the yield ratio binned in $x_{b j}$ using different cuts (Cut2 - Cut5) to the yield ratio using the reference cut are very small for different kinematic settings. Two red dashed lines show $\pm 5 \%$ variation from one. 


\subsection{Yield ratio for ${ }^{40} \mathrm{Ca}$ and ${ }^{48} \mathrm{Ca}$}

\section{Yield ratio for ${ }^{40} \mathrm{Ca}$ and ${ }^{48} \mathrm{Ca}$ using initial cross section model}

The procedure for extracting the yield ratio and cross section was fully checked, and worked well with the ${ }^{12} \mathrm{C}$ target. The same procedure can be used to extract the ${ }^{40} \mathrm{Ca}$ and ${ }^{48} \mathrm{Ca}$ yield ratios. As discussed in the Section 4.6.3, in the cross section model XEMC the total cross section is a sum of the quasi-elastic and deep inelastic contributions. The quasi-elastic contribution is calculated from a scaling function $F(y)$ which is extracted from data for each target. Subsequently a new $F(y)$ fit is performed on the extracted cross section to obtained an iterated cross section model which is used as input to the cross section extraction for next iteration. This process is repeated until good agreement between data and the model was achieved. However there are no preexisting in this kinematic region for ${ }^{40} \mathrm{Ca}$ and ${ }^{48} \mathrm{Ca}$ targets. This means that we did not have a good starting $F(y)$ cross section model for these targets. The scaling function for aluminum was used in the first iteration for both ${ }^{40} \mathrm{Ca}$ and ${ }^{48} \mathrm{Ca}$ which is used as a starting point of this analysis.

Figures 5.18 and 5.19 show the yield ratio (data/MC) for ${ }^{40} \mathrm{Ca}$ and ${ }^{48} \mathrm{Ca}$ for both $\mathrm{E}^{\prime}$ and $x_{b j}$ binning using the initial cross section model. As expected, the yield ratio is not close to 1 yet since the initial cross section model can not describe data well. Even though the yield ratio in the overlap region is in very good agreement indicating that the acceptance is well described. This yield ratio used to obtain the initial extracted cross section for ${ }^{40} \mathrm{Ca}$ and ${ }^{48} \mathrm{Ca}$, as shown in Figures 5.20, 5.21. The uncertainty shown is only statistical.

\section{Refitting $\mathbf{F}(\mathbf{y})$ fitting for ${ }^{40} \mathrm{Ca}$ and ${ }^{48} \mathrm{Ca}$ data}

After the initial cross section of ${ }^{40} \mathrm{Ca}$ and ${ }^{48} \mathrm{Ca}$ was extracted, it was used to obtain a new $F(y)$ fit. The $F^{d a t a}(y)$ can be extracted from data by taking the initial cross 

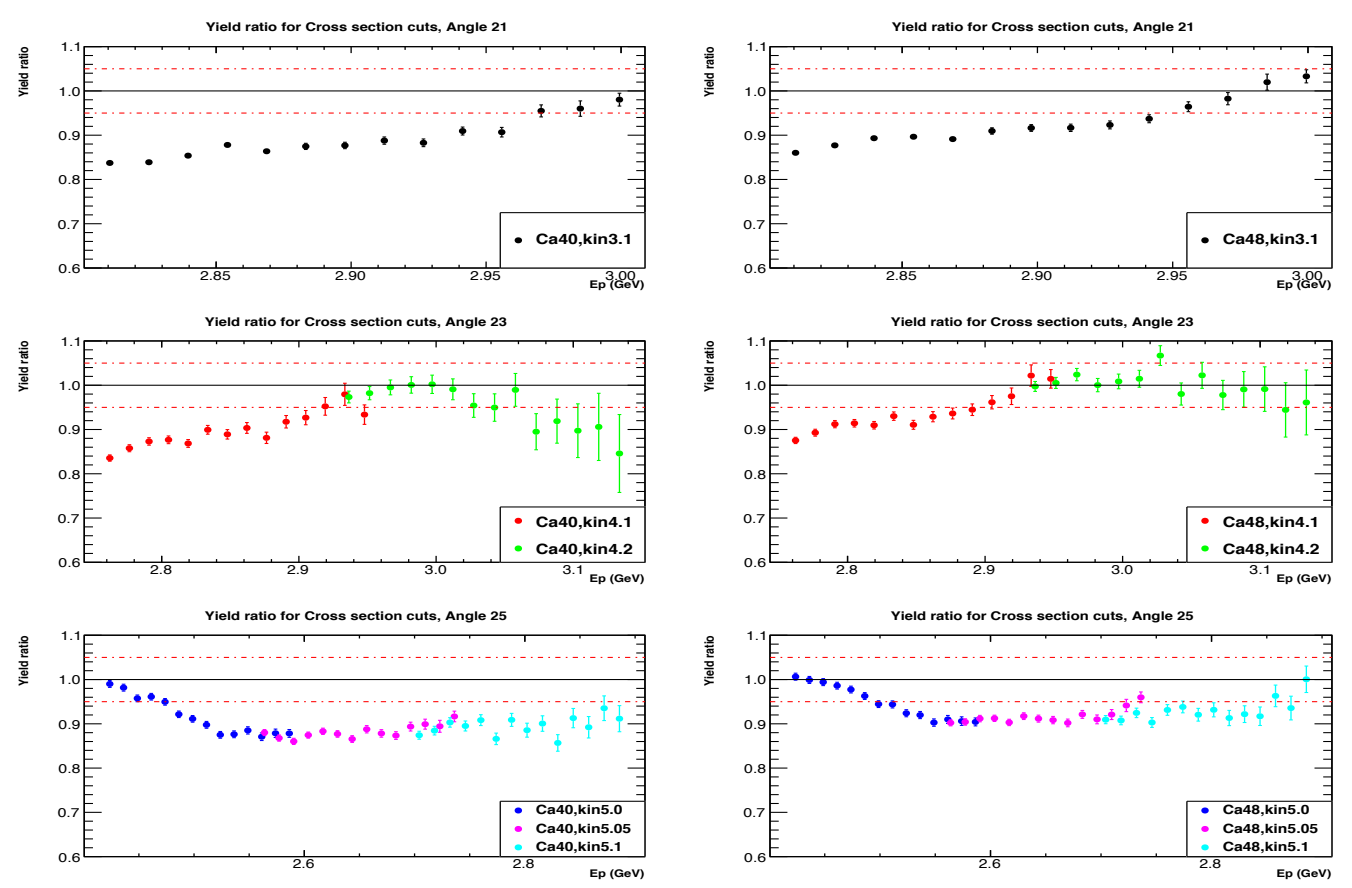

Figure 5.18: The ${ }^{40} \mathrm{Ca}$ (left), ${ }^{48} \mathrm{Ca}$ (right) yield ratio as a function of $\mathrm{E}^{\prime}\left(\mathrm{E}_{p}\right)$ using the initial cross section model. Two red dashed lines show $\pm 5 \%$ variation from one.
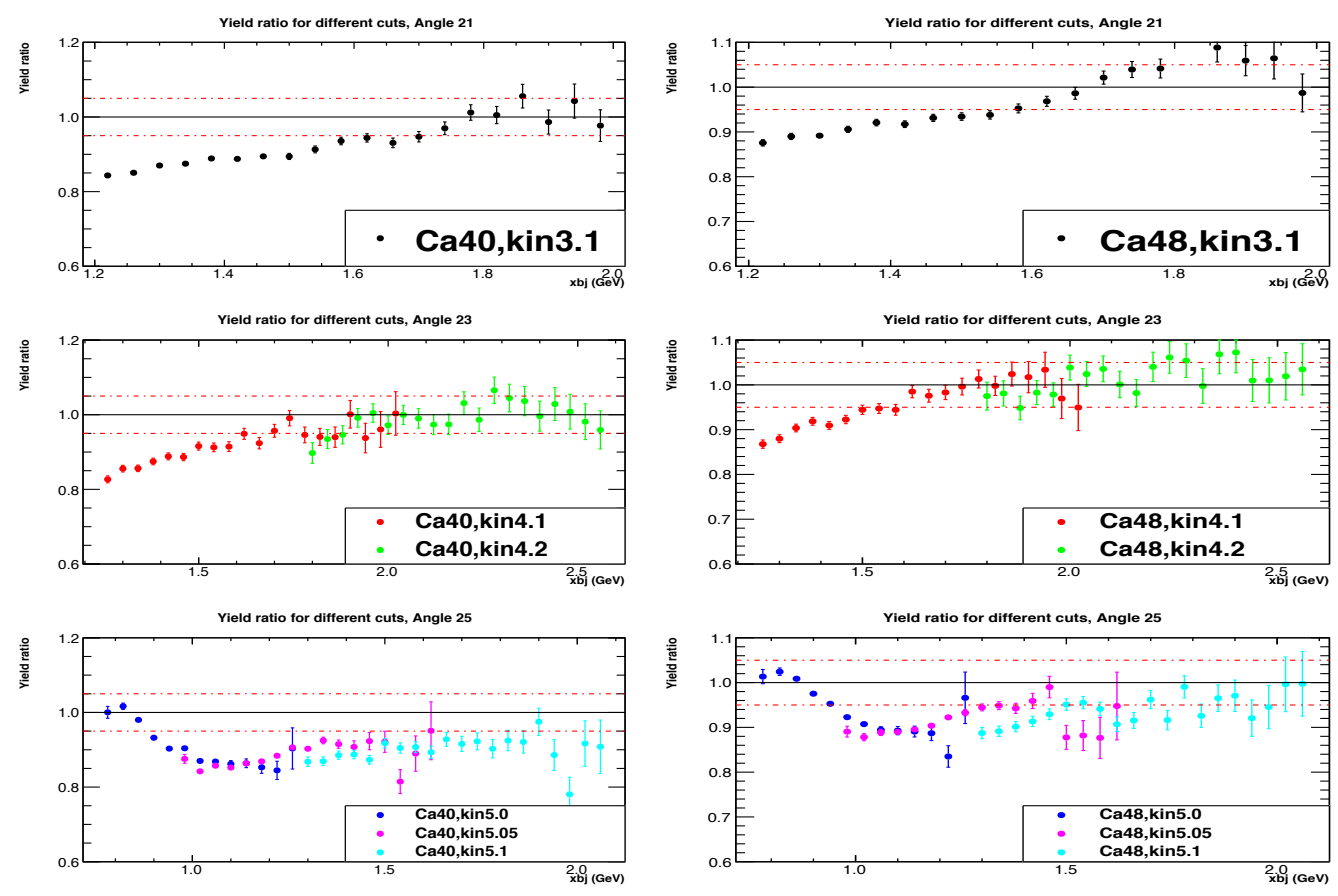

Figure 5.19: The ${ }^{40} \mathrm{Ca}$ (left), ${ }^{48} \mathrm{Ca}$ (right) yield ratio as function of $\mathrm{x}_{b j}$ using the initial cross section model. Two red dashed lines show $\pm 5 \%$ variation from one. 
Single foil $\mathrm{Ca} 40, \mathrm{E0}=3.356 \mathrm{GeV}$, Theta: $21,23,25$

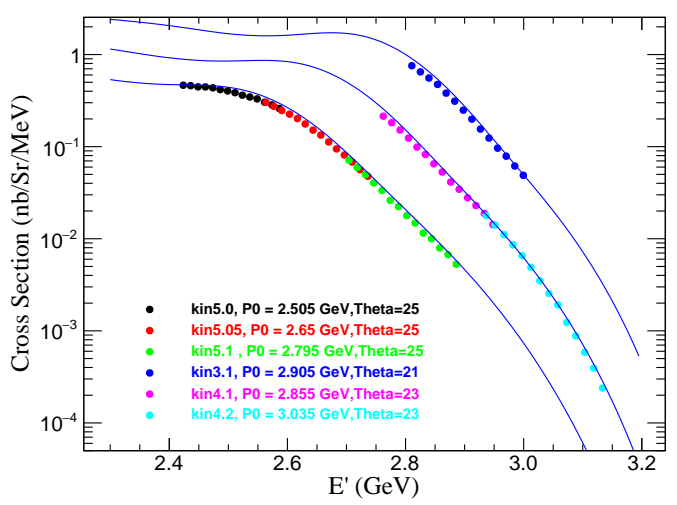

Single foild $\mathrm{Ca} 40, \mathrm{E} 0=3.356 \mathrm{GeV}$, Theta: $21,23,25$

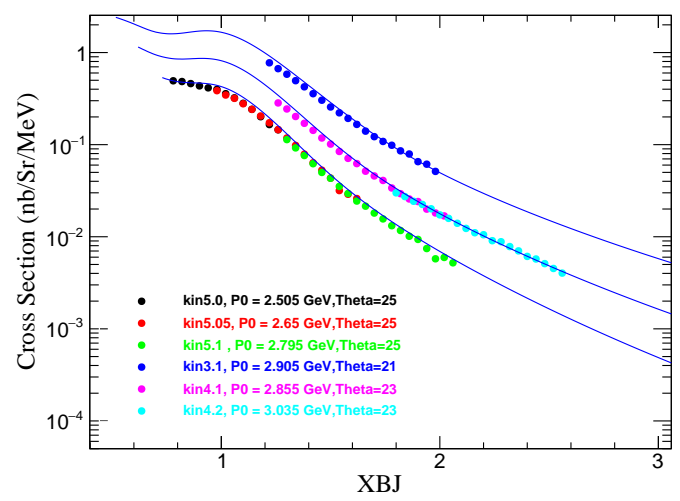

Figure 5.20: The initial extracted ${ }^{40} \mathrm{Ca}$ cross section using the initial cross section model. The left plot is the cross section as a function of $\mathrm{E}^{\prime}$ (Gev). The right plot is the cross section as a function of $\mathrm{x}_{b j}$.
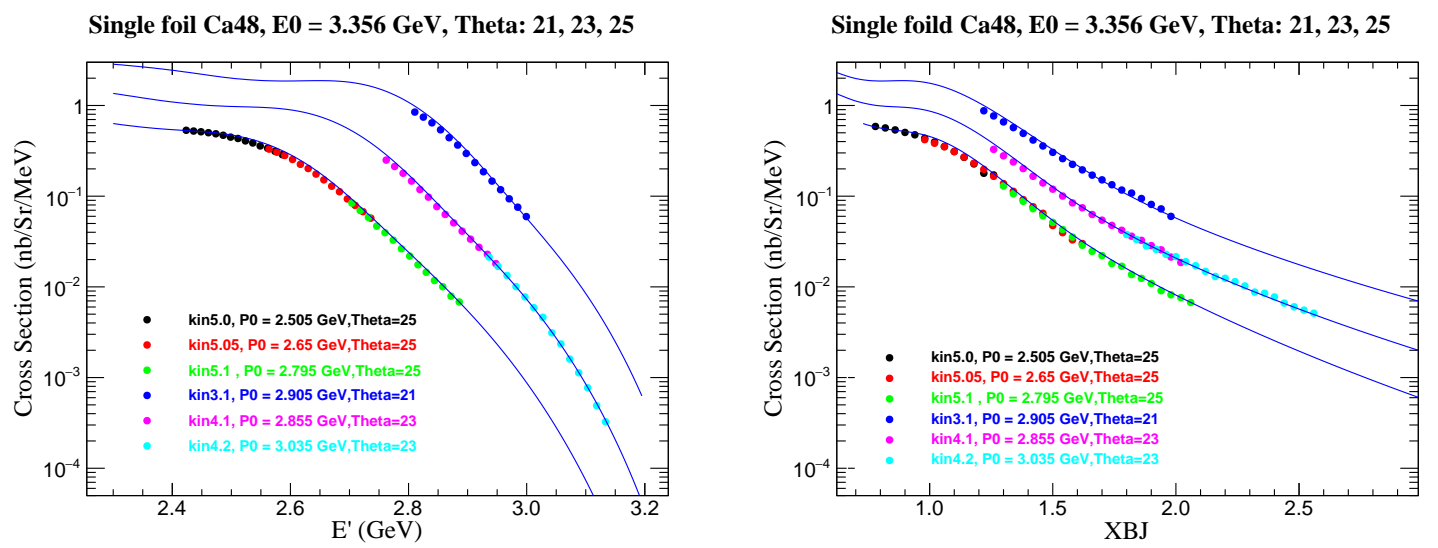

Figure 5.21: The initial extracted ${ }^{48} \mathrm{Ca}$ cross section using the initial cross section model. The left plot is the cross section as a function of $\mathrm{E}^{\prime}(\mathrm{GeV})$. The right plot is the cross section as a function of $\mathrm{x}_{b j}$.

section, subtracting the inelastic contribution (calculated by the inelastic part of XEMC cross section model) and dividing out the kinematic factor and the sum of electron-nucleon cross section as shown in Equation 5.10 .

$$
F^{\text {data }}(y)=\frac{\left(\sigma^{\text {data }}-\sigma_{\text {dis }}^{\text {xemc }}\right)}{\left(Z \sigma_{p}+N \sigma_{n}\right)} \cdot \frac{q}{\sqrt{M^{2}+(\vec{p}+\vec{q})^{2}}},
$$


A parameterization of the scaling function $F(y)$, given in equation 5.11 , is used to perform a fit on the $F^{\text {data }}(y)$. The fit provides the new parameters to update cross section model and is used for the next iteration to get updated yield ratio and updated extracted cross section.

$$
F(y)=\left(f_{0}-B\right) \frac{\alpha^{2} e^{-(a y)^{2}}}{\alpha^{2}+y^{2}}+B e^{-(b y)^{2}}
$$

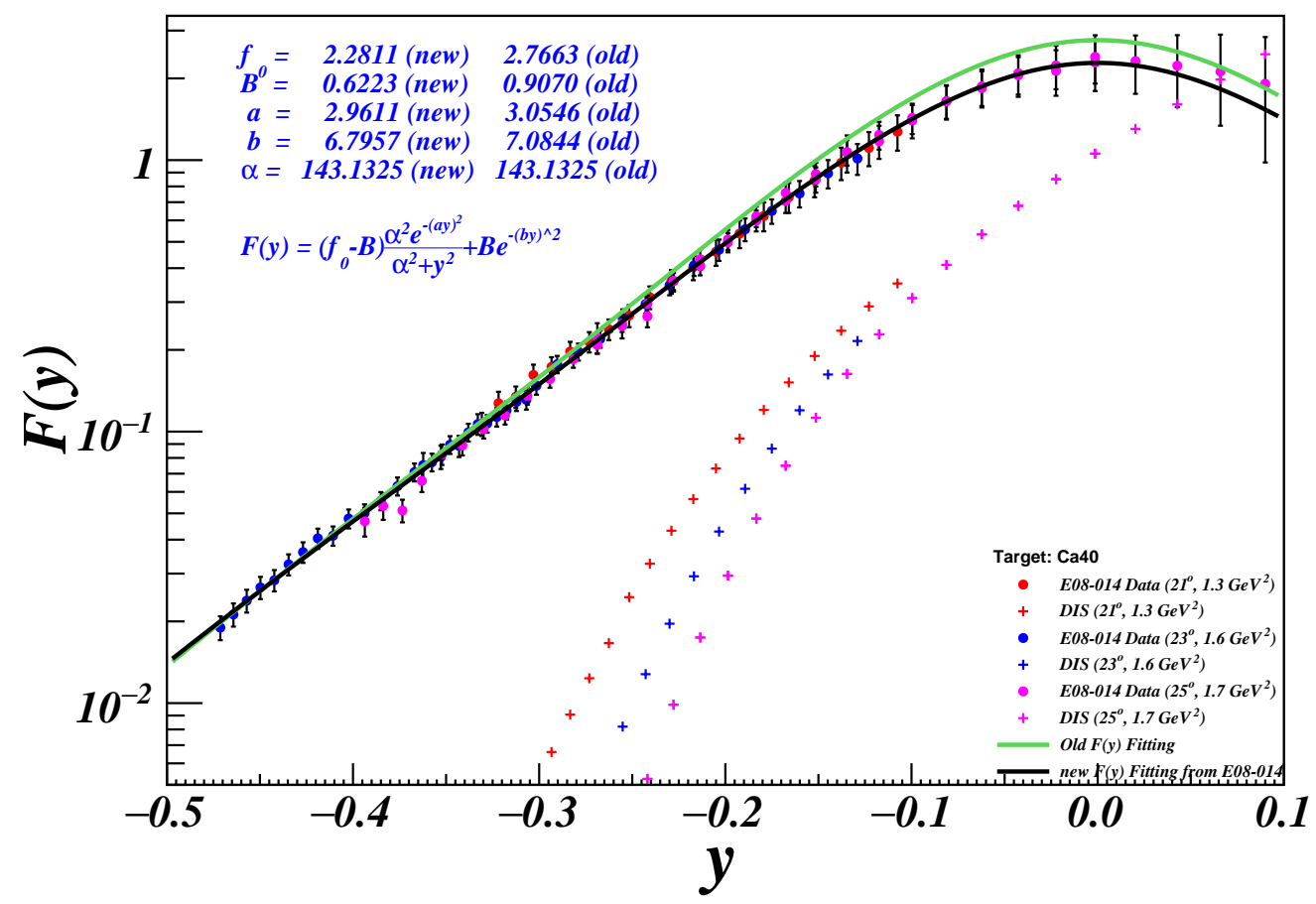

Figure 5.22: The new $\mathrm{F}(\mathrm{y})$ fit on the initial extracted ${ }^{40} \mathrm{Ca}$ cross section. The solid points are the $\mathrm{F}(\mathrm{y})$ extracted from data, different colors represent different kinematic settings. The crosses are the DIS contribution corresponding to each kinematic settings. The old parameters in the legend are for initial cross section model while the new parameters, obtained from the fit, are for updated cross section model.

\section{Yield ratio of ${ }^{40} \mathrm{Ca}$ and ${ }^{48} \mathrm{Ca}$ using updated cross section model}

Using the updated cross section model for ${ }^{40} \mathrm{Ca}$ and ${ }^{48} \mathrm{Ca}$, the updated yield ratios (data/simulation) are obtained as shown in Figures 5.24 and 5.25 . The yield ratio 


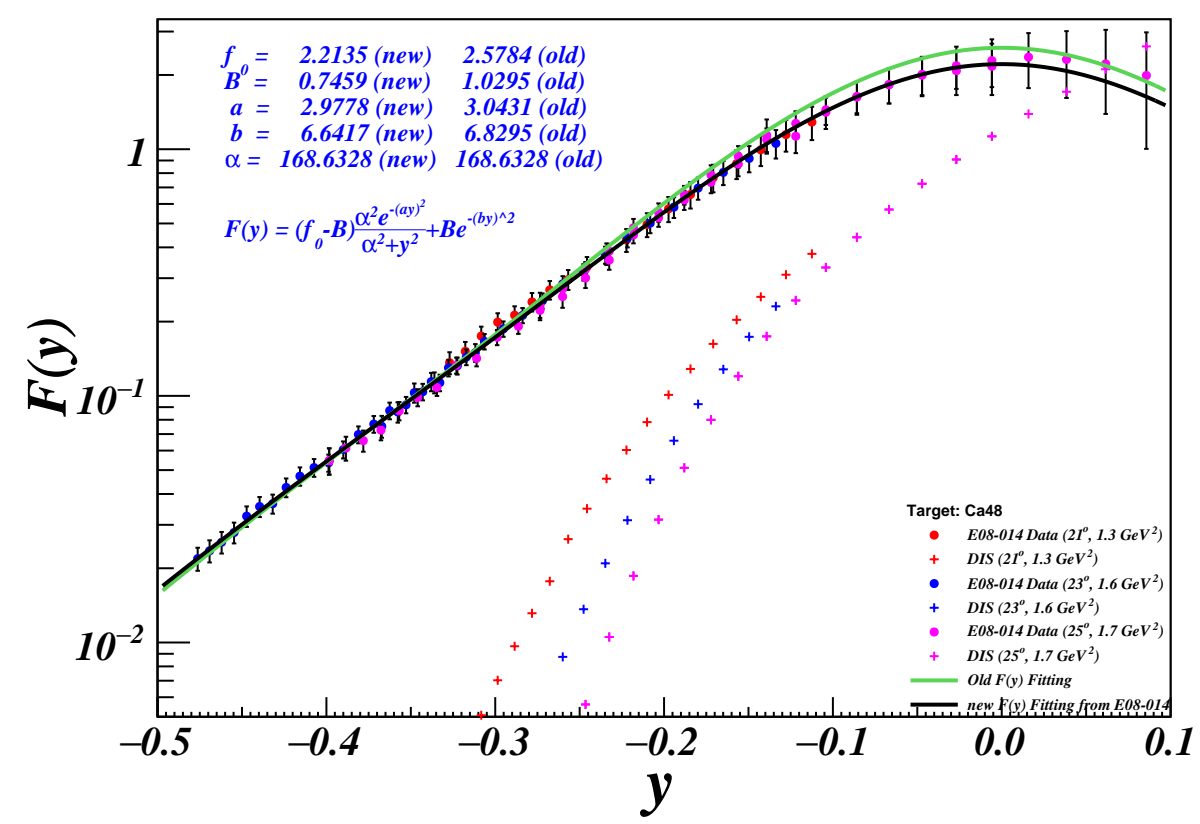

Figure 5.23: The new $\mathrm{F}(\mathrm{y})$ fit on the initial extracted ${ }^{48} \mathrm{Ca}$ cross section. The solid points are the $\mathrm{F}(\mathrm{y})$ extracted from data, different colors represent different kinematic settings. The crosses are the DIS contribution corresponding to each kinematic settings. The old parameters in the legend are for initial cross section model while the new parameters, obtained from the fit, are for updated cross section model.

is much closer to 1 compared to the yield ratio using the initial cross section model after only one iteration. In addition, the good agreement in the overlapping regions was retained. At the lowest $Q^{2}$ kinematic setting, kin3.1, the cross section may suffer a larger final state interaction, but this behavior is consistent for both ${ }^{40} \mathrm{Ca}$ and ${ }^{48} \mathrm{Ca}$ and would cancel in the cross section ratio. In the end, another iteration is needed to improve the cross section model. The variation of the extracted cross section using a newly iterated cross section model can be used to study the model dependence. 

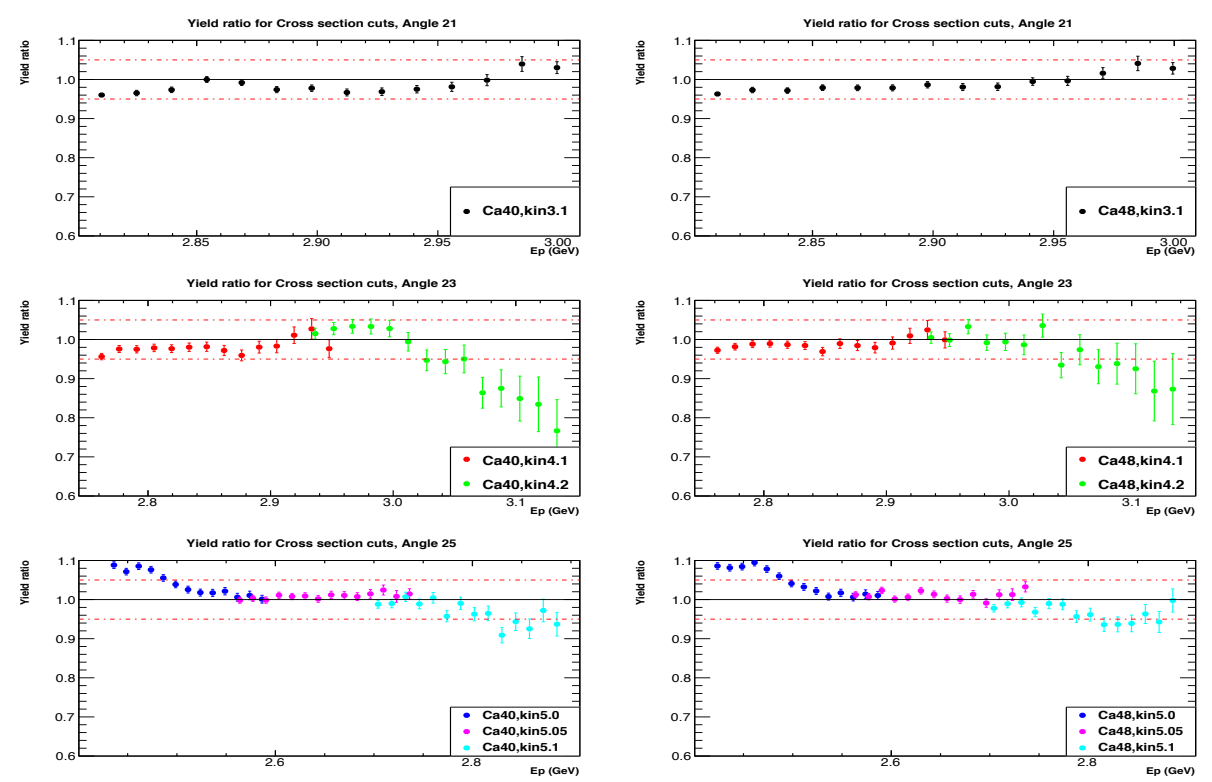

Figure 5.24: The ${ }^{40} \mathrm{Ca}$ (left), ${ }^{48} \mathrm{Ca}$ (right) yield ratio as a function of $\mathrm{E}^{\prime}\left(\mathrm{E}_{p}\right)$ using the iterated cross section model. The yield ratio is much closer to 1 compared to the yield ratio using the initial cross section. The two red dashed lines show $\pm 5 \%$ variation from one.
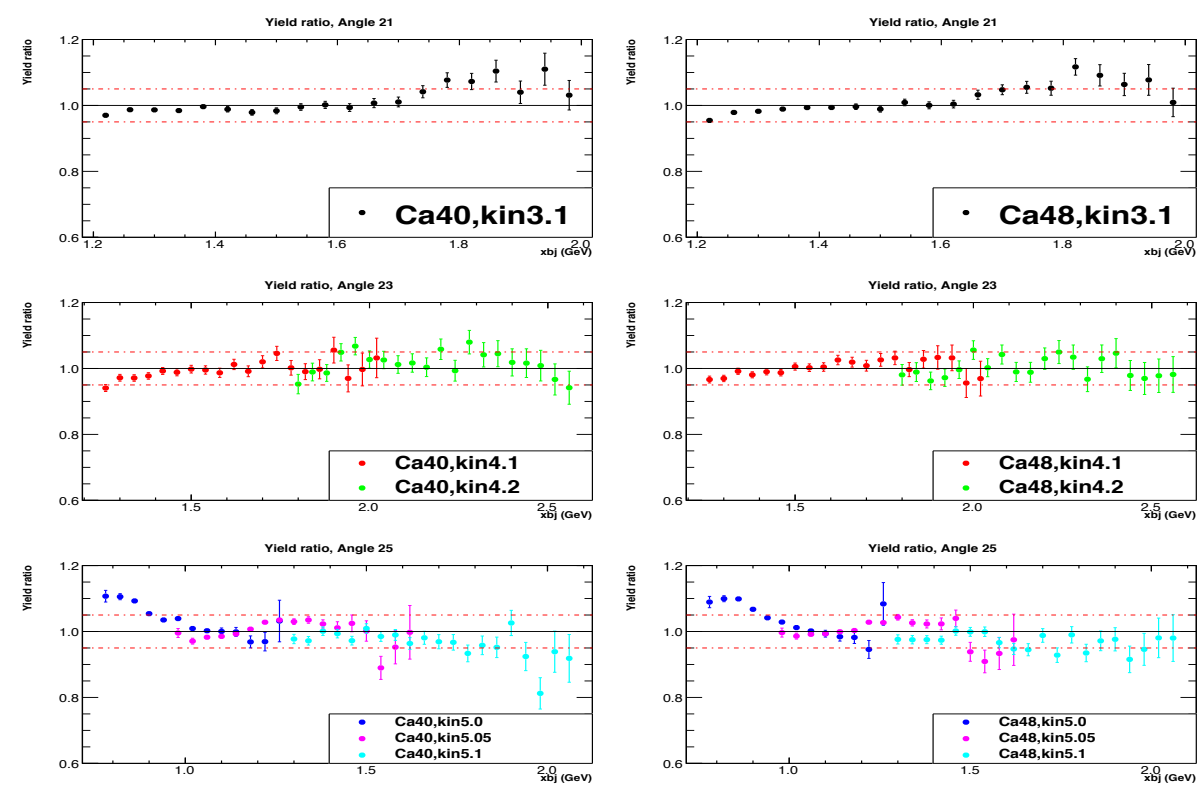

Figure 5.25: The ${ }^{40} \mathrm{Ca}$ (left), ${ }^{48} \mathrm{Ca}$ (right) yield ratio as function of $\mathrm{x}_{b j}$ using the iterated cross section model. The yield ratio is much closee to 1 compared to the yield ratio using the initial cross section. The two red dashed line show $\pm 5 \%$ variation from one 


\subsection{Model dependence}

The yield ratio method for extracting cross section depends on the cross section model. After each new $\mathrm{F}(\mathrm{y})$ fitting, the iterated cross section is obtained and is used to get the updated yield ratio and then updated extracted cross section. To see cross section model dependence effects, a comparison of the extracted cross sections using the initial cross section model and the first iterated cross section model is performed. First of all, we can compare the difference in these two cross section models for each target, ${ }^{40} \mathrm{Ca}$ and ${ }^{48} \mathrm{Ca}$, as shown in Figures 5.26 and 5.27. The difference between the two cross section models is at level of $10 \%-15 \%$ at quasi-elastic peak and at level of $20 \%$ for large values of $E^{\prime}$. Even so, this difference in the cross section models has very small impact in the extracted cross sections as seen below
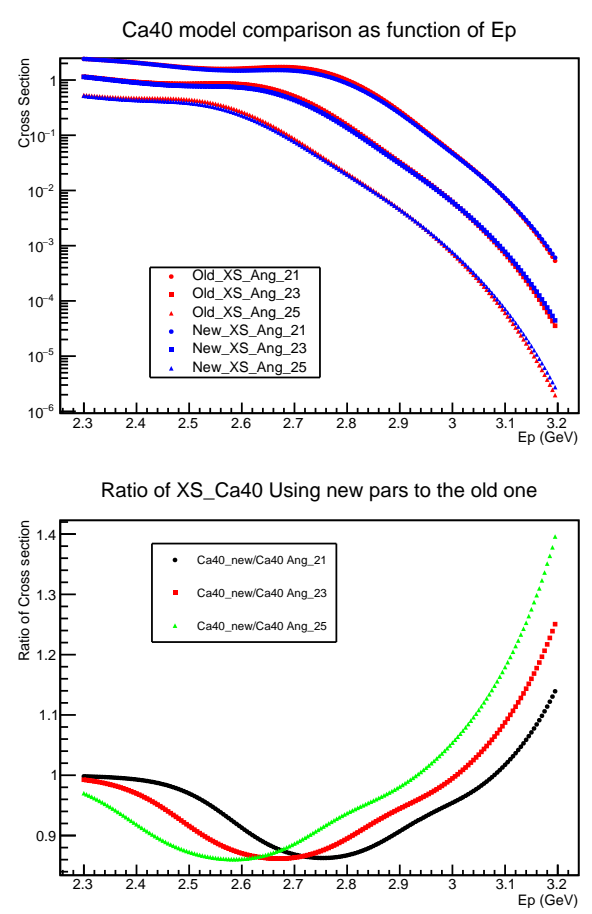
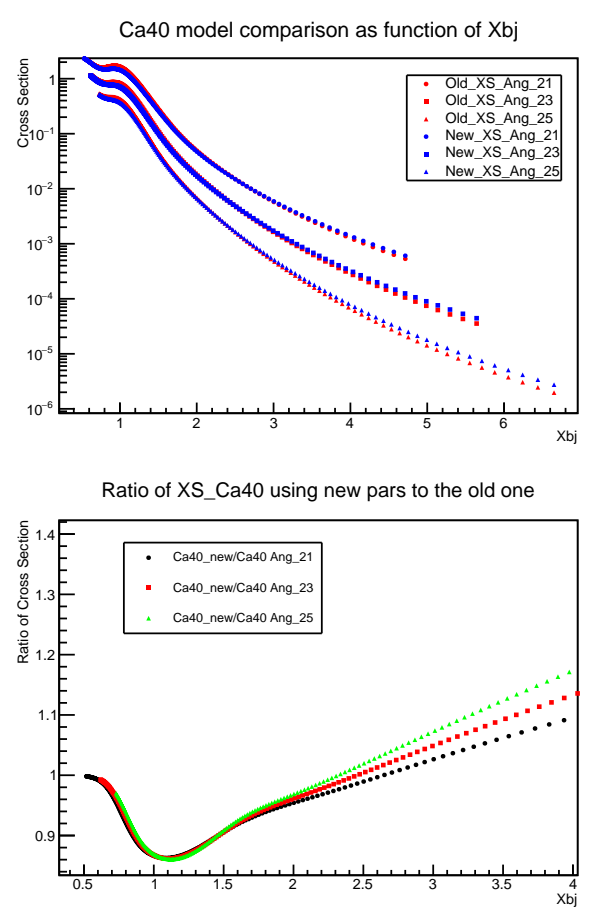

Figure 5.26: Top plots:The initial (old) and the first iterated (new) ${ }^{40} \mathrm{Ca}$ cross section model comparison for $\theta=21^{\circ}, 23^{\circ}, 25^{\circ}$ as a function of $\mathrm{E}^{\prime}\left(\mathrm{E}_{p}\right)$ or $\mathrm{x}_{b j}$, respectively. The bottom plots: the ratio new/old of cross section models which shows difference at the level of 10\%-15\% around the quasi-elastic peak and 20\%-30\% for large $E^{\prime}$ values and less when binned in $x_{b j}$. 

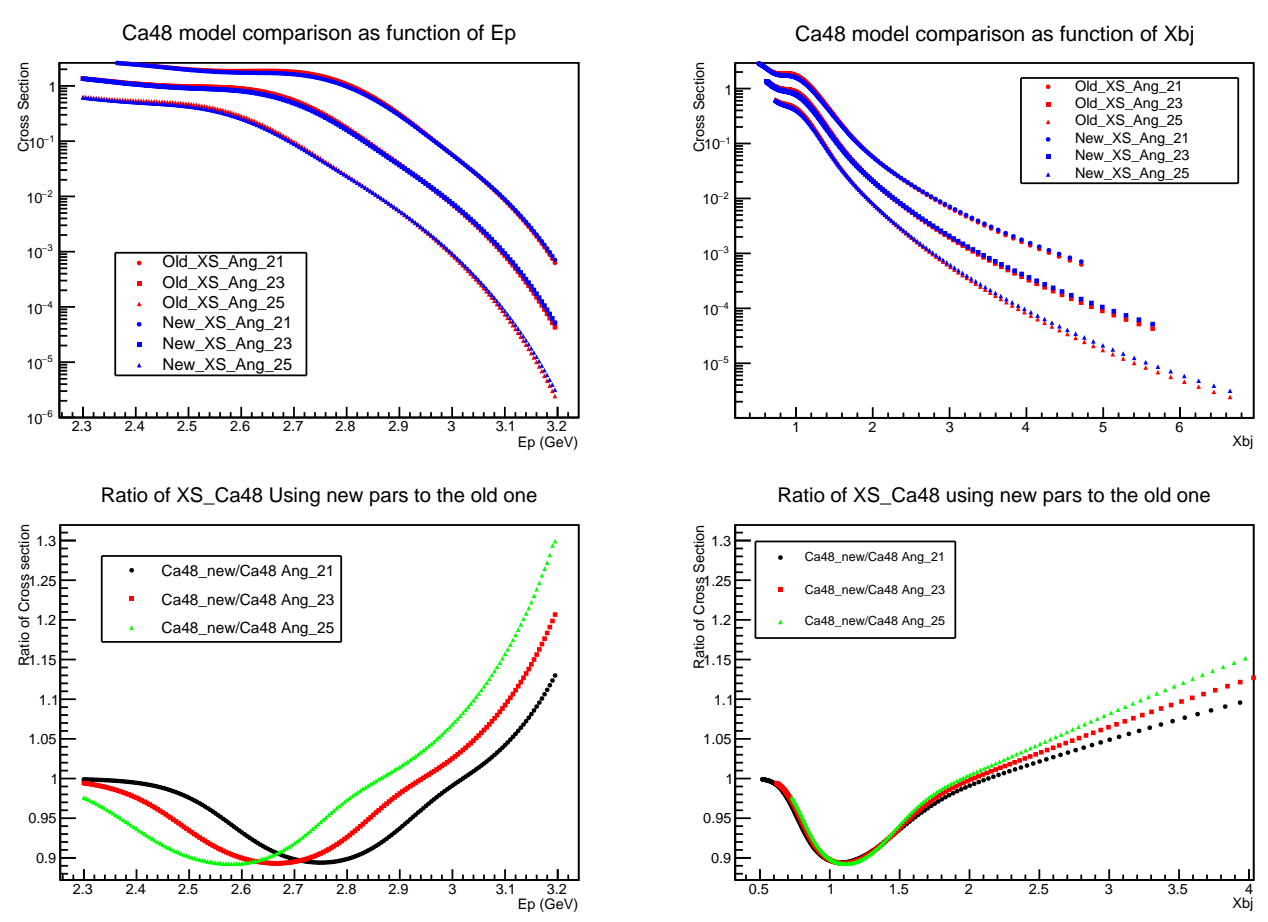

Figure 5.27: Top plots:The initial (old) and the first iterated (new) ${ }^{48}$ Ca cross section model comparison for $\theta=21^{\circ}, 23^{\circ}, 25^{\circ}$ as a function of $\mathrm{E}^{\prime}\left(\mathrm{E}_{p}\right)$ or $\mathrm{x}_{b j}$, respectively. The bottom plots: the ratio new/old of cross section models which shows differences at the level of $10 \%-15 \%$ around the quasi-elastic peak and $20 \%-30 \%$ for large $E^{\prime}$ values.

Figures 5.28 and 5.29 show the variation in the extracted cross section when using the initial cross section model and the first iterated cross section model as a function of $E^{\prime}$ and $x_{b j}$ for both ${ }^{40} \mathrm{Ca}$ and ${ }^{48} \mathrm{Ca}$. The variation in the extracted cross sections is at level of $2 \%$. But the overall deviation is much smaller, the RMS scatter of the points is about $0.5 \%$. So this is a reasonable upper limit for the model dependence. One thing to be aware of is that the variation in the $\mathrm{x}_{b j}$ binning is smaller compared to the $\mathrm{E}^{\prime}$ binning is explained through Figure 5.30 . When the cross section is binned in $\mathrm{E}^{\prime}$, each bin covers a large range of $\mathrm{x}_{b j}$ which means that the cross section change significantly across the bin and that is why it is much more sensitive to the model for $\mathrm{E}^{\prime}$ binning (see Figure 5.30 , left plot). When the cross section binned in $\mathrm{x}_{b j}$, the cross section is much flatter across the bin which makes it less sensitive to the model (see Figure 5.30, right plot). Detailed studies of the model dependence effects are found 
in the Refs. 98 100].
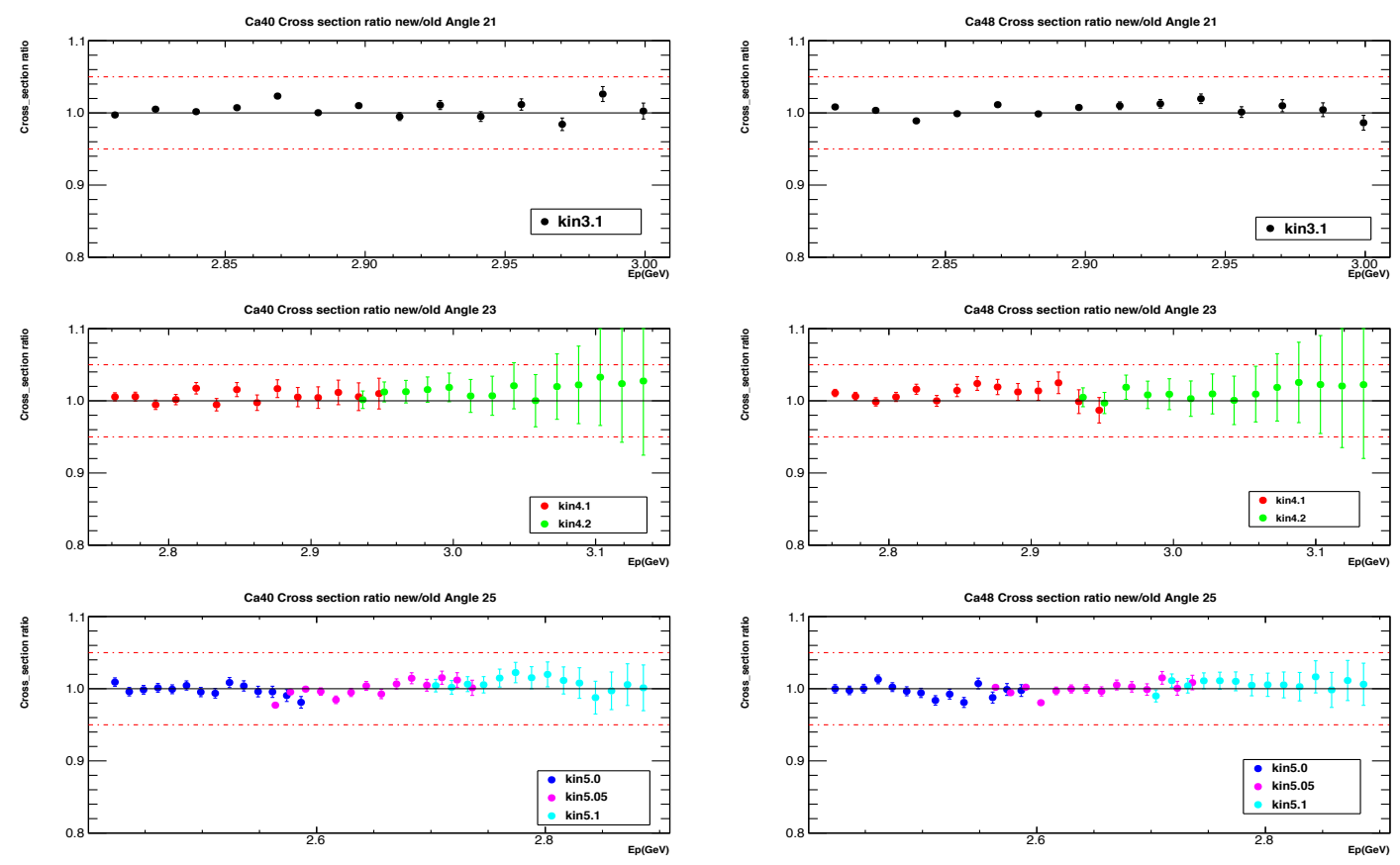

Figure 5.28: The ratio of the extracted cross using the first iterated cross section model to the extracted cross section using initial cross section model section as a function of $E^{\prime}$ for different kinematic settings. The left plots are for ${ }^{40} \mathrm{Ca}$ while the right plots are for ${ }^{48} \mathrm{Ca}$. Two red dashed lines in each plot shows $\pm 5 \%$ variation from one.

If we do additional iterations on cross section model the uncertainty from the model dependence can be reduced. But for this state of the analysis a $0.5 \%$ uncertainty from the model dependence on the cross section point to point is acceptable. The $\mathrm{F}(\mathrm{y})$ fit was performed separately on ${ }^{48} \mathrm{Ca}$ and ${ }^{40} \mathrm{Ca}$ which implies that uncertainty from model dependence can be slightly different for these two nuclei. There can be some cancellation when we take the cross section ratio ${ }^{48} \mathrm{Ca} /{ }^{40} \mathrm{Ca}$. So it is reasonable to apply $0.5 \%$ uncertainty from model dependence point to point for the cross section ratio ${ }^{48} \mathrm{Ca} /{ }^{40} \mathrm{Ca}$ as well 87 . 

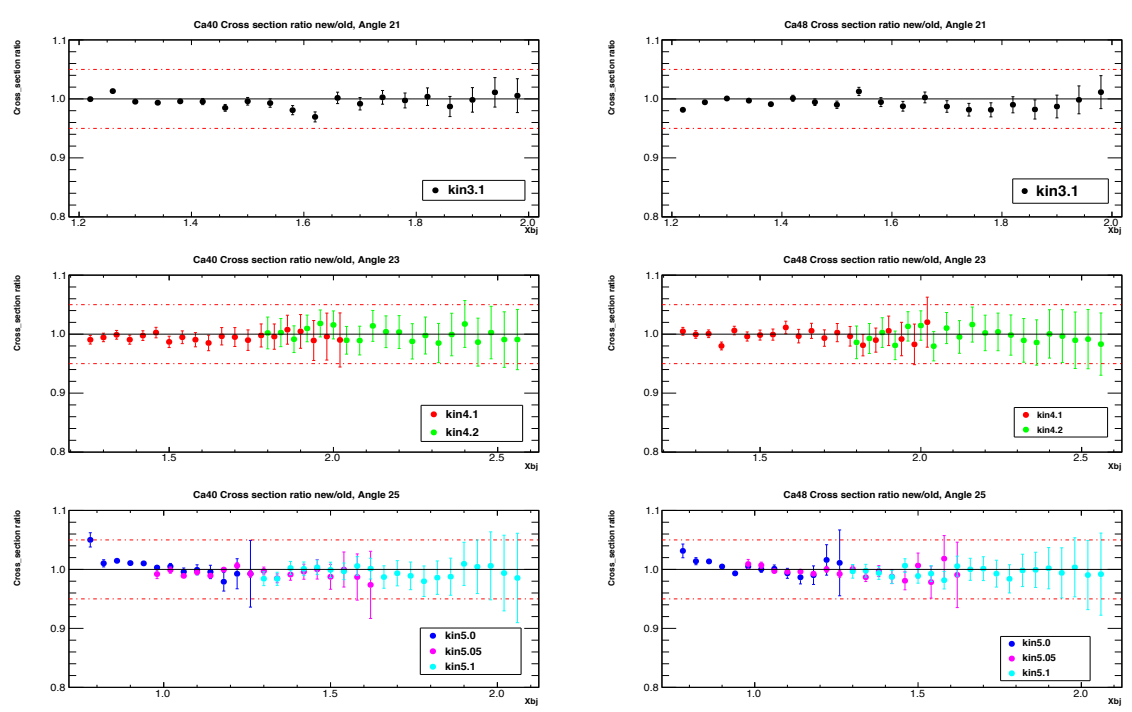

Figure 5.29: The ratio of the extracted cross using the first iterated cross section model to the extracted cross section using initial cross section model section as a function of $x_{b j}$ for different kinematic settings. The left plots are for ${ }^{40} \mathrm{Ca}$ while the right plots are for ${ }^{48} \mathrm{Ca}$. Two red dashed lines in each plot shows $\pm 5 \%$ variation from one.
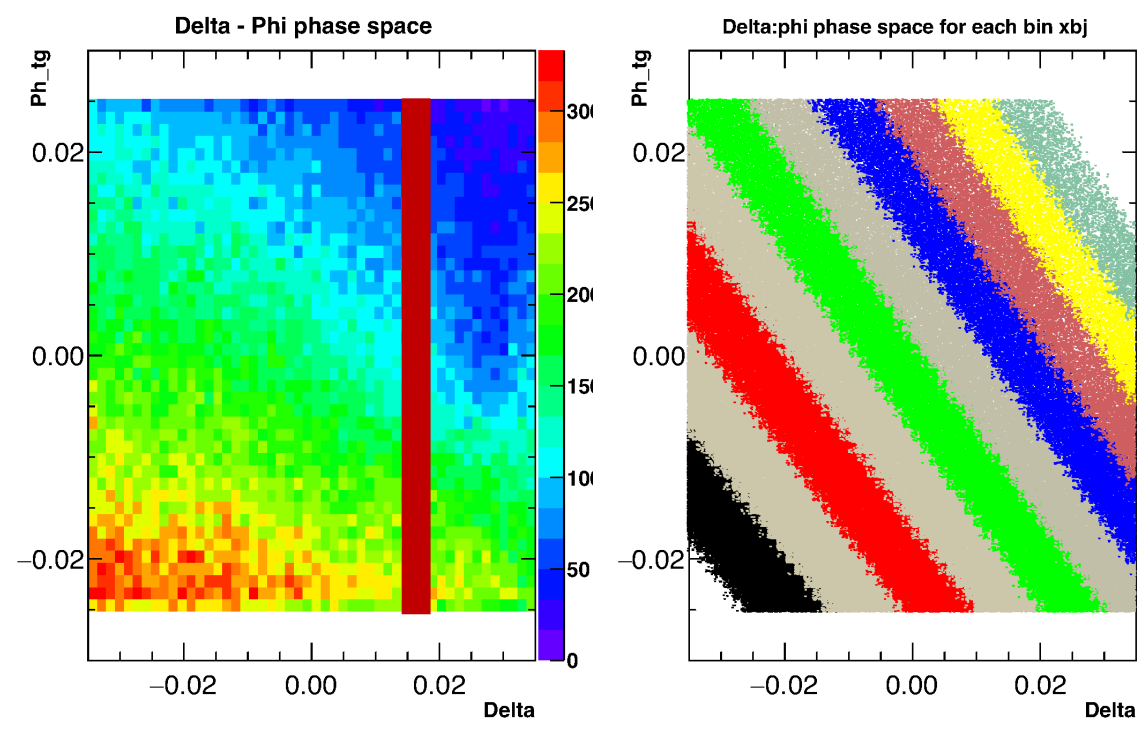

Figure 5.30: The left plot shows the $\left(d p: \phi_{t g}\right)$ phase space after the selected cuts of the cross section analysis. The red band presents the phase space corresponding to a $\mathrm{dp}\left(\mathrm{E}^{\prime}\right)$ bin. The cross section changes a lot crossing the $d p$ bin. The right plot shows the $\left(d p: \phi_{t g}\right)$ phase space after the selected cuts of the cross section analysis within the selected range of $\mathrm{x}_{b j}$. Each color band represents a phase space of each $\mathrm{x}_{b j}$ bin. The cross section is much flatter crossing the $\mathrm{x}_{b j}$ bin compared to the $\mathrm{E}^{\prime}$ bin. 


\subsection{Systematic uncertainty estimation}

The extracted cross section and the cross section ratio were corrected for many effects. The systematic uncertainly associated with each correction needs to be studied. The systematic uncertainty of the acceptance correction, the model dependence as well as the trigger and PID efficiencies have been studied in this analysis. The remaining uncertainty studies are the radiative corrections and the target density. The expensive ${ }^{48} \mathrm{Ca}$ was well made and its density was determined with high precision $(0.1 \%)$. The density of the less expensive target ${ }^{40} \mathrm{Ca}$ was not well defined and we estimate an uncertainty of $\sim 1 \%$. A conservative estimate for the radiative correction is $1.5 \%$. The detail studies of these uncertainties will be updated for eventual publication. A summary of normalization (norm) and point-to-point (pt-pt) uncertainty of each correction for both absolute cross-section $(\delta \sigma / \sigma)$ and cross-section ratio $(\delta R / R)$ can be found in Table 5.2 with the marker $(*)$ indicating an on-going study.

Table 5.2: Systematic Uncertainty summary

\begin{tabular}{||c|c|c|c|c||}
\hline \hline Systematic & $\delta \sigma / \sigma($ Norm $)$ & $\delta \sigma / \sigma(\mathrm{pt}-\mathrm{pt})$ & $\delta R / R($ Norm $)$ & $\delta R / R(\mathrm{pt}-\mathrm{pt})$ \\
\hline Acceptance dependence & $2 \%$ & $1.5 \%$ & - & $0.2 \%$ \\
\hline Model dependence & - & $0.5 \%$ & - & $0.5 \%$ \\
\hline Tracking efficiency & $1 \%$ & $0.3 \%$ & - & $0.2 \%$ \\
\hline Cer efficiency & $0.3 \%$ & $0.1 \%$ & - & $0.1 \%$ \\
\hline E/p efficiency & $0.3 \%$ & $0.1 \%$ & - & $0.1 \%$ \\
\hline Target density* & $1 \%$ & - & $1 \%$ & - \\
\hline Beam Charge* $^{*}$ & $0.5 \%$ & - & - & $0.5 \%$ \\
\hline radiative correction* $^{*}$ & $1.5 \%$ & - & - & $0.5 \%$ \\
\hline Total & $2.9 \%$ & $1.6 \%$ & $1 \%$ & $0.9 \%$ \\
\hline \hline
\end{tabular}




\section{Chapter 6}

\section{Results and discussion}

In this chapter the preliminary absolute cross section results are presented for three targets: ${ }^{12} \mathrm{C},{ }^{40} \mathrm{Ca}$ and ${ }^{48} \mathrm{Ca}$. In addition, the cross section ratios for ${ }^{48} \mathrm{Ca} /{ }^{40} \mathrm{Ca}$ are presented. Due to the absence of theoretical predictions of the cross section for these heavy nuclei we are necessarily limited in our discussion. However, the precision of these data will provide the possibility for comparison to advanced calculations as they become available. The cross section ratios do however provide an opportunity for some discussion as the expectation is that the ratios, in the region where $2 \mathrm{~N}$ SRCs should dominate, will be largely determined by whether 2N-SRCs are isospin dependent or isospin independent.

\subsection{Absolute Cross section}

The absolute cross-sections of ${ }^{40} \mathrm{Ca}$ and ${ }^{48} \mathrm{Ca}$ have been measured for the first time in a region of kinematics $\left(Q^{2}>1\right.$ and the $\left.x_{b j}>1\right)$ where SRC should play an important role. At a minimum these results will allow the construction of precise models for these two nuclei for future studies. In addition, by exploiting the connection between the longitudinal scaling function $F(y)$ and the momentum distribution $n(k)$ a comparison can be made with predictions whose starting point is the NN potential [21]. 


\section{${ }^{12} \mathrm{C}$ absolute cross section}

(a) ${ }^{12} \mathrm{C}$ cross section as function of $E_{p}$

Single foil 12C, E0= $3.356 \mathrm{GeV}$, Theta:21, 23, 25

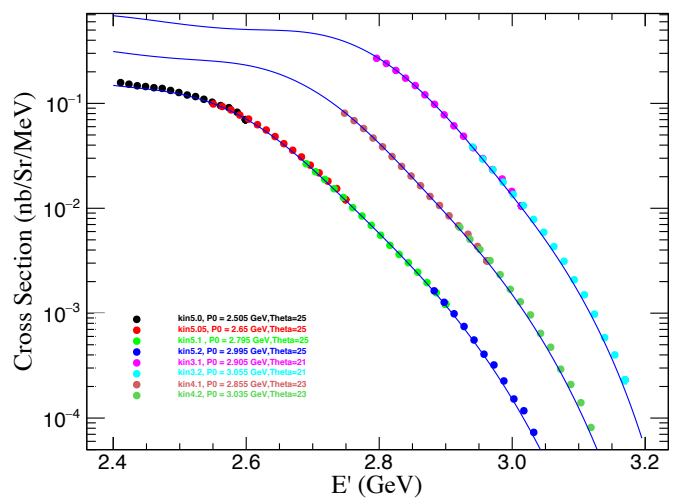

(b) ${ }^{12} \mathrm{C}$ cross section as function of $x_{b j}$

Single foil 12C, E0= $3.356 \mathrm{GeV}$, Theta:21, 23, 25

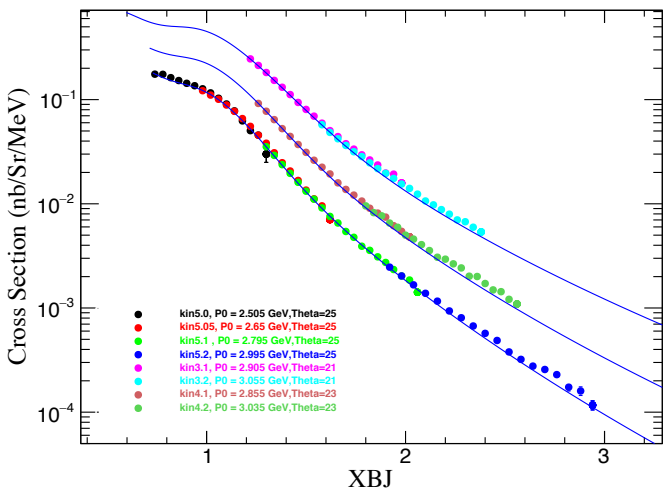

Figure 6.1: ${ }^{12} \mathrm{C}$ absolute cross-section as a function of $E^{\prime}$ (left panel) and $x_{b j}$ (right panel) for different kinematic settings. The solid lines represent the model and the points represent the E08014 data.

\section{${ }^{40} \mathrm{Ca}$ and ${ }^{48} \mathrm{Ca}$ absolute cross section}

(a) ${ }^{40} \mathrm{Ca}$ cross-section as function of $E_{p}$

Single foil Ca40, E0 = 3.356 GeV, Theta: $21,23,25$

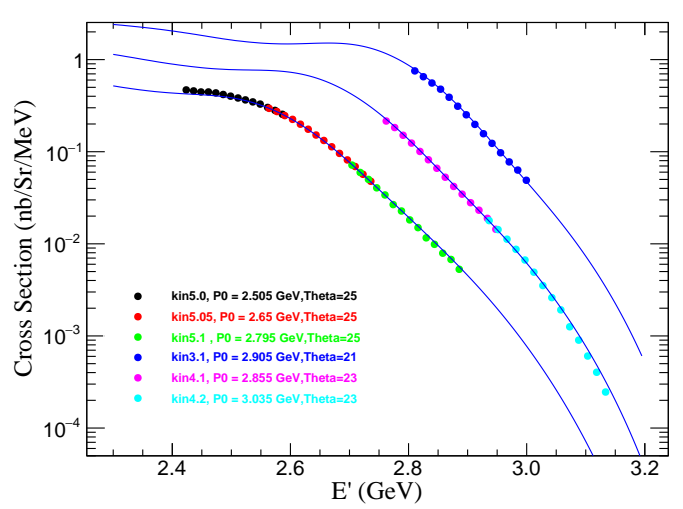

(b) ${ }^{40} \mathrm{Ca}$ cross-section as function of $x_{b j}$ Single foild Ca40, E0 $=3.356 \mathrm{GeV}$, Theta: $21,23,25$

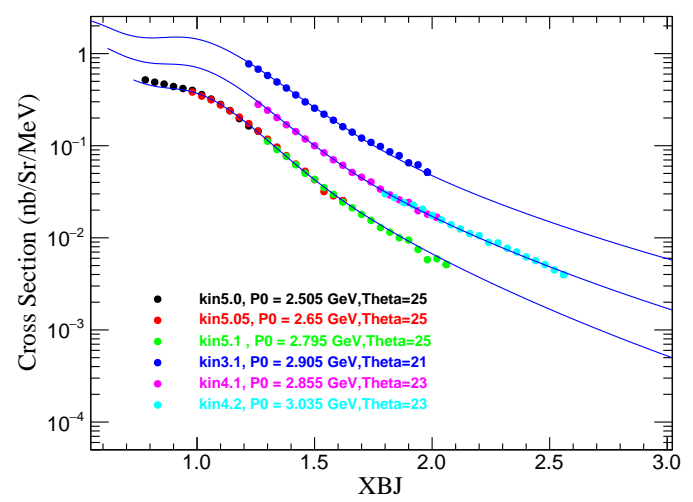

Figure 6.2: ${ }^{40} \mathrm{Ca}$ absolute cross-section as a function of $E^{\prime}$ (left panel) and $x_{b j}$ (right hand panel) for different kinematic settings. The solid lines represent the cross section model and the points represent the E08014 data. 
(a) ${ }^{48} \mathrm{Ca}$ cross-section as function of $E_{p}$

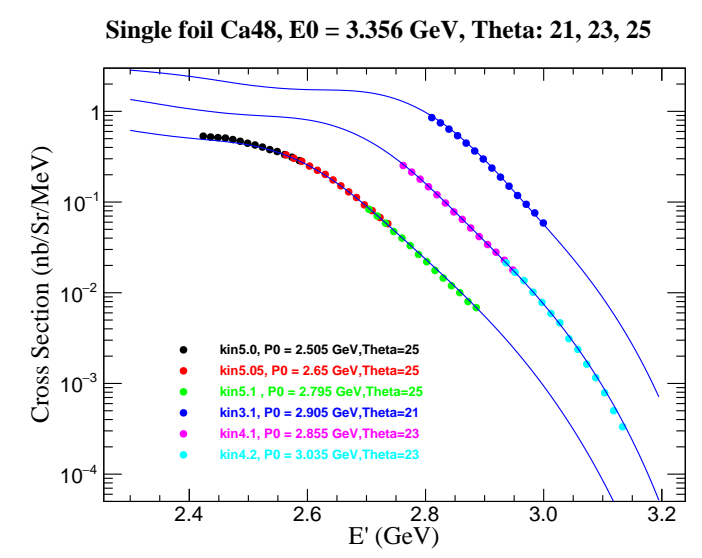

(b) ${ }^{48}$ Ca cross-section as function of $x_{b j}$

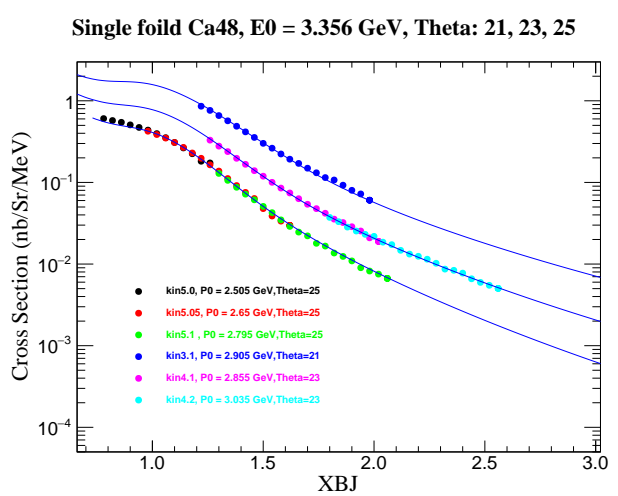

Figure 6.3: ${ }^{48} \mathrm{Ca}$ cross-section as function of $E^{\prime}$ (left panel) and $x_{b j}$ (right panel) for different kinematic settings. The solid lines represent the cross-section model and the points represent the E08014 data.

\subsection{Cross section ratio ${ }^{48} \mathrm{Ca} /{ }^{40} \mathrm{Ca}$}

The focus of this thesis is the isospin-dependence of $2 \mathrm{~N}$ SRCs using the cross section ratio ${ }^{48} \mathrm{Ca} /{ }^{40} \mathrm{Ca}$ per-nucleon. As discussed in Section 2.4 , with the isospinindependent assumption, the ratio of neutron to proton in SRCs is equal to the $\mathrm{N} / \mathrm{Z}$ ratio of nucleus. In the kinematics of E08014, the cross section of electron-proton scattering $\left(\sigma_{p}\right)$ is about 3 times the electron-neutron scattering $\left(\sigma_{n}\right)$. In the isospinindependent assumption, the cross section ratio per-nucleon from ${ }^{48} \mathrm{Ca}$ to ${ }^{40} \mathrm{Ca}$ can be expressed as [41]:

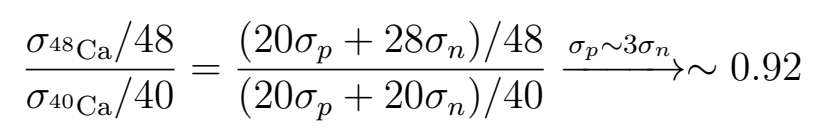

In the case of the isospin-dependence, it is not possible to give a rock-solid estimate for the ratios due to the complexity of large nuclei. In the proposal 41 for E08014 a guesstimate, based on the total possible combination of np pairs, $(Z * N)$, was given:

$$
\frac{\sigma^{48} \mathrm{Ca} / 48}{\sigma^{40} \mathrm{Ca} / 40}=\frac{(20 \cdot 28) / 48}{(20 \cdot 20) / 40}=1.17
$$


Subsequent to this a theoretical calculation [57] took into account the role of isospin-dependence effect in short-range correlations for a range of nuclei. The results have already been shown in Figure 2.17 in Chapter 2. By picking off the value of $\frac{N_{p n}}{(N Z)}$ from Figure 2.17, for ${ }^{40} \mathrm{Ca}$ and ${ }^{48} \mathrm{Ca}$, multiplying by their associated $(N \times Z)$ and then dividing by $\frac{48}{40}$, the prediction for isospin-dependence of the ratio of cross section per nucleon ${ }^{48} \mathrm{Ca} /{ }^{40} \mathrm{Ca}$ is around 1 ,

$$
\frac{\sigma^{48} \mathrm{Ca} / 48}{\sigma_{40} \mathrm{Ca} / 40} \sim 1
$$

The following figures show the cross section ratio of ${ }^{48} \mathrm{Ca} /{ }^{40} \mathrm{Ca}$ per nucleon in this analysis for different angle settings. The discussion of these results is in the next section.
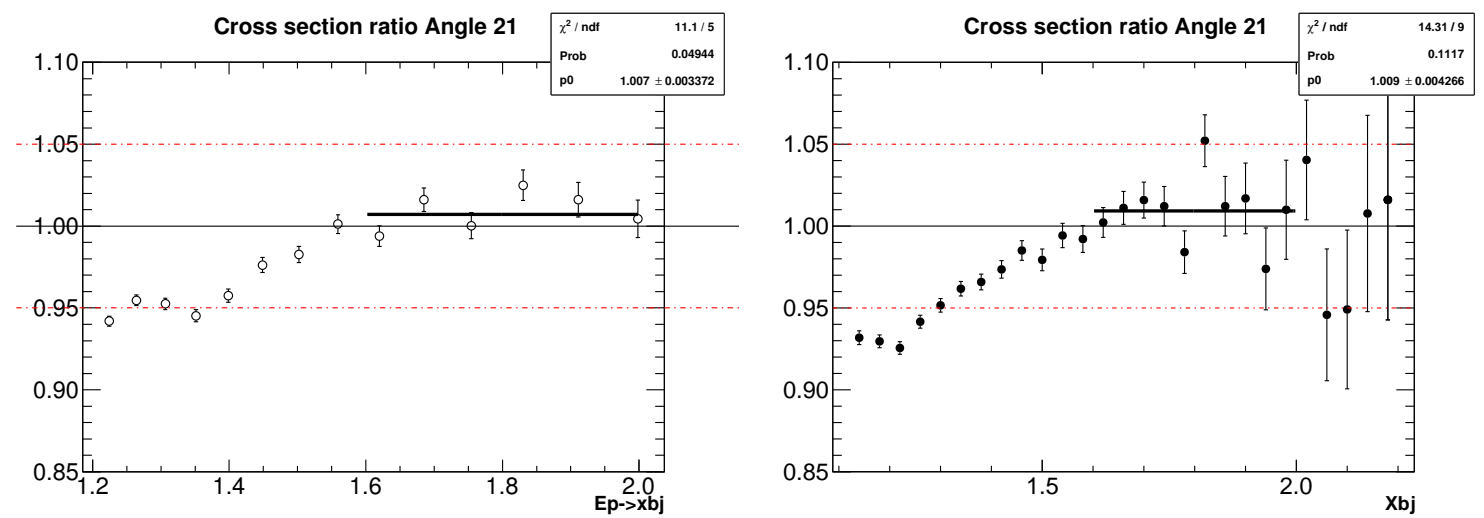

Figure 6.4: The cross-section ratio ${ }^{48} \mathrm{Ca} /{ }^{40} \mathrm{Ca}$ per nucleon for $\theta_{0}=21^{\circ}, Q^{2} \sim 1.3$ $\mathrm{GeV}^{2}$, binned in $E^{\prime}$ converted to $x_{b j}$ (left panel) and binned in $x_{b j}$ (right panel). The short black line shows the linear fit on the data in the region $1.6<x_{b j}<2$. 

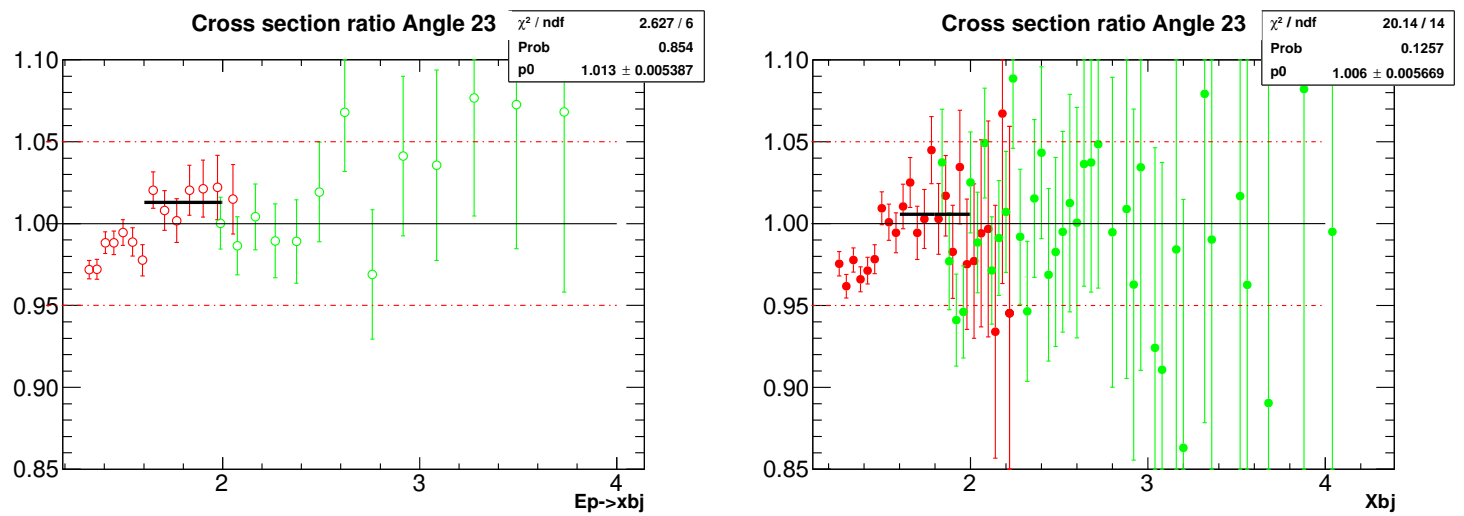

Figure 6.5: The cross-section ratio ${ }^{48} \mathrm{Ca} /{ }^{40} \mathrm{Ca}$ per nucleon for $\theta_{0}=23^{\circ}, Q^{2} \sim 1.5$ $\mathrm{GeV}^{2}$, binned in $E^{\prime}$ converted to $x_{b j}$ (left hand side) and binned in $x_{b j}$ (right hand side). The short black line shows the linear fit on the data in the region $1.6<x_{b j}<2$.
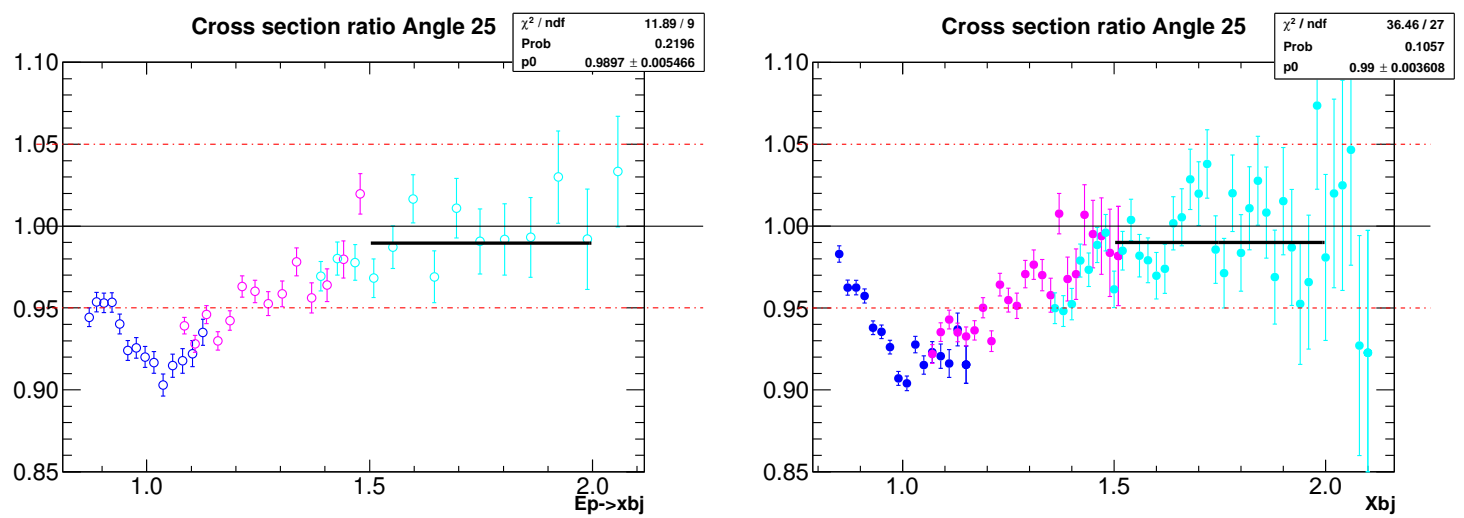

Figure 6.6: The cross-section ratio ${ }^{48} \mathrm{Ca} /{ }^{40} \mathrm{Ca}$ per nucleon for $\theta_{0}=25^{\circ}, Q^{2} \sim 1.7$ $\mathrm{GeV}^{2}$, binned in $E^{\prime}$ converted to $x_{b j}$ (left panel) and binned in $x_{b j}$ (right panel). The short black line shows the linear fit on the data in the region $1.5<x_{b j}<2$.

\subsection{Discussion}

The cross section ratio per-nucleon ${ }^{48} \mathrm{Ca} /{ }^{40} \mathrm{Ca}$ shows a $\mathrm{SRC}$ plateau in the region $1.5<x_{b j}<2$. The value of this plateau is very close to 1 for different kinematic settings. This result is consistent with the theoretical prediction of the cross section ratio per-nucleon in the isospin-dependent case [57] as well as the observed isospin-dependence results from different nuclei using the exclusive data in Ref [47]. Therefore, these results can be considered as the first evidence of 2N SRCs isospin- 
dependence using inclusive scattering data. It allows the unequivocal statement that proposal for experiment made a significant overestimate for the fraction of np pairs participating in SRCs (see Section 2.4 [41].

In addition, the calculation in Ref. [101] provided the values of $a_{2}(A)$ for ${ }^{40} \mathrm{Ca}$ and ${ }^{48}$ Ca. See Table 6.1.

Table 6.1: Theoretical $a_{2}(A)$ calculation for ${ }^{40} \mathrm{Ca}$ and ${ }^{48} \mathrm{Ca} 101,102$

\begin{tabular}{||c|c|c|c||}
\hline \hline${ }^{2} \mathrm{H}$ & 1.128 & ${ }^{40} \mathrm{Ca}$ & 1.637 \\
\hline${ }^{4} \mathrm{He}$ & 1.327 & ${ }^{48} \mathrm{Ca}$ & 1.629 \\
\hline${ }^{9} \mathrm{Be}$ & 1.384 & ${ }^{56} \mathrm{Fe}$ & 1.638 \\
\hline${ }^{12} \mathrm{C}$ & 1.435 & ${ }^{108} \mathrm{Ag}$ & 1.704 \\
\hline${ }^{16} \mathrm{O}$ & 1.527 & ${ }^{197} \mathrm{Au}$ & 1.745 \\
\hline${ }^{27} \mathrm{Al}$ & 1.545 & ${ }^{208} \mathrm{~Pb}$ & 1.741 \\
\hline
\end{tabular}

The $a_{2}(A)$ can be obtained by taking ratio of the numbers in Table 6.1 subtracted by one because they are the extra effect caused by SRC on top of the mean field value of 1 .

$$
a_{2}\left({ }^{40} \mathrm{Ca}\right)=\frac{\sigma^{40} \mathrm{Ca} / 40}{\sigma^{2} \mathrm{D} / 2}=\frac{(1.637-1)}{(1.128-1)}=4.97
$$

and:

$$
a_{2}\left({ }^{48} \mathrm{Ca}\right)=\frac{\sigma^{48} \mathrm{Ca} / 48}{\sigma^{2} \mathrm{D} / 2}=\frac{(1.629-1)}{(1.128-1)}=4.92
$$

The per-nucleon cross section ratio from this calculation is:

$$
\frac{\sigma^{48} \mathrm{Ca} / 48}{\sigma^{40} \mathrm{Ca} / 40}=4.92 / 4.97=0.989
$$

Since these two nuclei have the same number of protons, this result has very interesting implications. It shows that increasing the number of neutrons will increase the fraction of protons prone to SRCs, otherwise the number of np pairs would be the same in the two nuclei and the cross section ratio per-nucleon will be equal to the ratio of nuclear masses $(40 / 48 \sim 0.83)$. This is consistent with the observation in Ref 103 that the average kinetic energy of protons is higher than the average kinetic 
energy of neutrons in neutron-rich nuclei, because the fraction of protons having high momentum is higher than the fraction of neutrons in the high momentum tail. The result can be understood in a simple way that, by increasing the number of neutrons, the total possible combination of np pairs $(Z * N)$ also increases which gives np pairs a higher probability to be a SRC np pair. A hand-waving argument to describe this relative probability can be expressed as:

$$
\frac{\sigma^{48} \mathrm{Ca} / 48}{\sigma^{40} \mathrm{Ca} / 40}=\frac{(20 \cdot 28) /(48 \cdot(48-1) / 2)}{(20 \cdot 20) /(40 \cdot(40-1) / 2)} \sim 0.97
$$

where $(20 \cdot 28)$ is the total number of combinations of np pairs and $(48 \cdot(48-1) / 2)$ is the total combination of two-nucleon pairs for ${ }^{48} \mathrm{Ca}$. It is similar for the ${ }^{40} \mathrm{Ca}$ case.

In a later stage of the analysis of E08014, ${ }^{2} \mathrm{D}$ data will allow the extraction of $a_{2}$, the $2 \mathrm{~N}$ SRC scaling factor, for both ${ }^{40} \mathrm{Ca}$ and ${ }^{48} \mathrm{Ca}$. This will be very useful in the study of the linear correlation of the EMC strength and SRCs as well as the density dependence of $a_{2}(A)$ 104.

\subsection{Outlook}

The isospin-dependence is one of the most important questions underlying the dynamics of SRCs. Pursuing it will give a clearer understanding of short-distance nuclear structure. SRCs imply high momenta, short distances and extremely high, though fleeting states of high density. Consequently, it will test the mechanism by which nucleon properties might be modified (the EMC effect for example) and expose whether they share a common origin. Furthermore, the SRCs are important for the interpretation of neutrino-oscillation measurements where nuclear structure is necessary for neutrino beam energy reconstruction 105. They are also relevant for the understanding of neutron rich systems such as neutron stars 33 . The SRCs may affect the equation of state of neutron stars and make it stiffer. 


\section{Bibliography}

[1] J. J. Thomson, Proceedings of the Royal Institution of Great Britain XV, 419 (1897).

[2] E. Rutherford, Philosophical Magazine 12 (68), 134 (1906).

[3] H. Geiger, Proceedings of the Royal Society A 83 (565), 492 (1910).

[4] C. James, Proceedings of the Royal Society A 136 (830), 692 (1932).

[5] B. R. Martin, Nuclear and Particle Physics an introduction (, 2008).

[6] R. B. Wiringa, R. Schiavilla, S. C. Pieper, and J. Carlson, Phys. Rev. C89, 024305 (2014), 1309.3794.

[7] L. Lapikas, Nucl. Phys. A553, 297c (1993).

[8] B. L. Cohen, Concepts of nuclear Physics (, 1971).

[9] O. Hen, Ph.D Thesis, Tel-Aviv University, 2015.

[10] Nuclear Shell Model, 2006, https://ocw.mit.edu/courses/ nuclear-engineering/22-101-applied-nuclear-physics-fall-2006/ lecture-notes/lec10.pdf.

[11] TUNL REU lectures. Nuclear Shell Model introduction to nuclear science, 2014, http://www.tunl.duke.edu/documents/public/REU2014/ Shell-Model-Lecture-2014.pdf.

[12] J. Mougey, Nuclear Physics A 335, 35 (1980).

[13] D. Role, Dissertation: Spectral function at high energy and momentum from (e,e'p) experiment, 2004.

[14] J. Arrington, D. W. Higinbotham, G. Rosner, and M. Sargsian, Prog. Part. Nucl. Phys. 67, 898 (2012), 1104.1196.

[15] R. B. Wiringa, V. G. J. Stoks, and R. Schiavilla, Phys. Rev. C 51, 38 (1995).

[16] R. Machleidt, Phys. Rev. C 63, 024001 (2001).

[17] R. V. Reid, Annals of Physics 50, 411 (1968). 
[18] C. Ciofi degli Atti, Phys. Rept. 590, 1 (2015).

[19] D. Day, Private communication.

[20] O. Benhar, C. Ciofi Degli Atti, S. Liuti, and G. SalmPhys. Lett. B177, 135 (1986).

[21] C. Ciofi degli Atti and S. Simula, Phys. Rev. C53, 1689 (1996), nuclth/9507024.

[22] D. W. Higinbotham, AIP Conf. Proc. 1374, 85 (2011), 1010.4433.

[23] J. Arrington, Ph.D Thesis, California Institute of Technology , 1998.

[24] O. Benhar, D. day, and I. Sick, Rev. Mod. Phys. 80, 189 (2008), nuclex/0603029.

[25] N. Fomin, Ph.D Thesis, UVA, 2008.

[26] E. Pace and G. Salme, Phys. Lett. 110B, 411 (1982).

[27] C. Ciofi Degli Atti, E. Pace, and G. Salme, Phys. Rev. C36, 1208 (1987).

[28] C. Ciofi degli Atti, E. Pace, and G. Salme, Phys. Rev. C43, 1155 (1991).

[29] T. De Forest, Nucl. Phys. A392, 232 (1983).

[30] D. Day et al., Phys. Rev. Lett. 43, 1143 (1979).

[31] S. Rock et al., Phys. Rev. C 26, 1592 (1982).

[32] L. L. Frankfurt and M. I. Strikman, Phys. Rept. 76, 215 (1981).

[33] L. Frankfurt, M. Sargsian, and M. Strikman, Int. J. Mod. Phys. A23, 2991 (2008), 0806.4412.

[34] M. M. Sargsian et al., J. Phys. G29, R1 (2003), nucl-th/0210025.

[35] N. Fomin, D. Higinbotham, M. Sargsian, and P. Solvignon, Ann. Rev. Nucl. Part. Sci. 67, 129 (2017), 1708.08581.

[36] D. B. Day, L. L. Frankfurt, M. M. Sargsian, and M. I. Strikman, (2018), 1803.07629.

[37] L. L. Frankfurt, M. I. Strikman, D. B. Day, and M. Sargsian, Phys. Rev. C48, 2451 (1993).

[38] CLAS, K. S. Egiyan et al., Phys. Rev. C68, 014313 (2003), nucl-ex/0301008.

[39] CLAS, K. S. Egiyan et al., Phys. Rev. Lett. 96, 082501 (2006), nucl-ex/0508026.

[40] L. L. Frankfurt and M. I. Strikman, Phys. Rept. 76, 215 (1981). 
[41] J. Arrington, D. Day, D. Higinbotham, and P. Solvignon, Three-nucleon short range correlations studies in inclusive scattering for $0.8<Q^{2}<2.8(\mathrm{GeV} / \mathrm{c})^{2}$, http://hallaweb.jlab.org/experiment/E08-014/, 2011.

[42] N. Fomin et al., Phys. Rev. Lett. 108, 092502 (2012), 1107.3583.

[43] Jefferson Lab Hall A, R. Shneor et al., Phys. Rev. Lett. 99, 072501 (2007), nucl-ex/0703023.

[44] R. Subedi et al., Science 320, 1476 (2008), 0908.1514.

[45] E. Piasetzky, M. Sargsian, L. Frankfurt, M. Strikman, and J. W. Watson, Phys. Rev. Lett. 97, 162504 (2006), nucl-th/0604012.

[46] R. Schiavilla, R. B. Wiringa, S. C. Pieper, and J. Carlson, Phys. Rev. Lett. 98, 132501 (2007), nucl-th/0611037.

[47] O. Hen et al., Science 346, 614 (2014), 1412.0138.

[48] European Muon, J. J. Aubert et al., Phys. Lett. 123B, 275 (1983).

[49] J. Gomez et al., Phys. Rev. D49, 4348 (1994).

[50] J. Seely et al., Phys. Rev. Lett. 103, 202301 (2009), 0904.4448.

[51] D. W. Higinbotham, J. Gomez, and E. Piasetzky, (2010), 1003.4497.

[52] O. Hen, D. W. Higinbotham, G. A. Miller, E. Piasetzky, and L. B. Weinstein, Int. J. Mod. Phys. E22, 1330017 (2013), 1304.2813.

[53] L. B. Weinstein et al., Phys. Rev. Lett. 106, 052301 (2011), 1009.5666.

[54] Z. Yez, Ph.D Thesis, UVA, 2013.

[55] Hall A, Z. Ye et al., Phys. Rev. C97, 065204 (2018), 1712.07009.

[56] D. W. Higinbotham and O. Hen, Phys. Rev. Lett. 114, 169201 (2015), 1409.3069.

[57] M. Vanhalst, J. Ryckebusch, and W. Cosyn, Phys. Rev. C86, 044619 (2012), 1206.5151 .

[58] J. Arrington, D. Day, D. Higinbotham, and P. Solvignon, Precision measurement of the isospin dependence in the $2 \mathrm{~N}$ and $3 \mathrm{~N}$ short range correlation region, https://www.jlab.org/exp_prog/proposals/11/PR12-11-112.pdf, 2018.

[59] J. Alcorn et al., Nucl. Instrum. Meth. A522, 294 (2004).

[60] Hall a beam line components, https://hallaweb.jlab.org/wiki/images/c/ ce/HallA_beamline_drawing.pdf. 
[61] P. Zhu, Beam position reconstruction for $\mathrm{g}_{2}^{p}$ experiment in the hall a at jefferson lab, http://hallaweb.jlab.org/experiment/g2p/technotes/bpm_ technote.pdf.

[62] Target configuration april 2011, http://hallaweb.jlab.org/experiment/ E08-014/analysis/HallA_Target_Configuration_Apr2011.pdf.

[63] Hall a cryo-target sceens, run period 1, http://hallaweb.jlab.org/halog/ log/html/1103_archive/110331235023.html.

[64] Hall a cryo-target sceens, run period 2, http://hallaweb.jlab.org/halog/ log/html/1105_archive/110513070119.html.

[65] K. G. Fissum et al., Nucl. Instrum. Meth. A474, 108 (2001).

[66] C. Hyde, Beam position studies for e093050, http://hallaweb.jlab.org/ publications/Technotes/files/2001/01-001.pdf.

[67] J. Huang, Extended target correction \& coincidence timing for tranversity, http://hallaweb.jlab.org/data_reduc/AnaWork2009/ Extended-Target-Corrections-Jin.pdf.

[68] B. Schmookler, Hall a 12 gev raster calibration, https://www.jlab.org/ indico/event/197/session/3/contribution/19/material/slides/0.pdf.

[69] C.Melissa, Ph.D Thesis, William and Mary University, 2016.

[70] N. Liyanage, Optics calibration of the hall a high resolution spectrometers using the new optimize, http://hallaweb.jlab.org/publications/Technotes/ files/2002/02-012.pdf.

[71] Y. Qiang, Ph.D Thesis, MIT, 2007.

[72] W. Chao, Ph.D Thesis, UVA, 2016.

[73] Y. Wang, Ph.D Thesis, William and Mary, 2017.

[74] Y. Wang, The gmp lhrs optics calibration, https://hallaweb.jlab.org/ dvcslog/Gmp/37.

[75] P. Solvignon, Bcm calibration for experiment e08014 report, https://userweb. jlab.org/ solvigno/src/e08014_analysis/xgt2_bcm_calib.pdf.

[76] D. Nguyen, Finalize the pid and tracking efficiency uncertainty, https:// hallaweb.jlab.org/dvcslog/Xgt2/346.

[77] B. Schmookler, Efficiency technote from GMP, 2018.

[78] J. Liu, Vdc multi-track efficiency study for e08-027, http://hallaweb. jlab.org/experiment/g2p/collaborators/jie/2013_09_24_multi_track_ notes/vdc_eff_technotes.pdf. 
[79] X. Zhan, Ph.D Thesis, MIT, 2010.

[80] O. Hansen, Hrs tracking algorithm analysis and improvements, https://userweb.jlab.org/ brads/bethe-heitler/VDC_tracking/ HRS-Tracking-HallAMtg.pdf.

[81] Hall a monte carlos, https://userweb.jlab.org/ yez/Work/SAMC/.

[82] H. Yao, Ph.D Thesis, Temple University, 2012.

[83] Technical drawing for hrs, http://hallaweb.jlab.org/12GeV/experiment/ E12-07-108/Publications/Technical/Spectrometer/HRS/.

[84] Snake package for hrs, https://github.com/MarkKJones/hallc-snake/ tree/master/hrs-examples.

[85] Xemc quasi-elastic cross section model, https://userweb.jlab.org/ yez/ Work/XEMC/.

[86] F. Benmokhtar, Ph.D Thesis, The State University of New Jersey, 2004.

[87] J. Arrington, Private communication.

[88] Spectrometer survey a1379, https://www.jlab.org/accel/survalign/ documents/dthalla/A1379.pdf.

[89] The run schedule of 4 experiments in the same time period, https: //www.jlab.org/exp_prog/experiment_schedule/2011/02-16/firm_ schedule2011.html.

[90] S. Stein et al., Phys. Rev. D12, 1884 (1975).

[91] W. Leo, Techniques for Nuclear and Particle Physics Experiment (, 1993).

[92] D. Griffiths, Introduction to Elementary Particles (, 1987).

[93] N. Fomin, The story how i extract the cross section, https://userweb.jlab. org/ fomin/xgt2/nadias_sigma.pdf.

[94] D. Nguyen, The local efficiency unique map calculation, https://hallaweb. jlab.org/dvcslog/Xgt2/323.

[95] D. Nguyen, The yield ratio comparison using two different ways to correct the local efficiency, https://hallaweb.jlab.org/dvcslog/Xgt2/322.

[96] P. Solvignon, Study of the acceptance cut effect on the cross section for e01-012, http://hallaweb.jlab.org/experiment/E01-012/reports/xs_cuts.pdf.

[97] D. Nguyen, Acceptance cuts effects on the cross section study, https:// hallaweb.jlab.org/dvcslog/Xgt2/333. 
[98] D. Nguyen, Model dependence study for ${ }^{12}$ c, https://hallaweb.jlab.org/ dvcslog/Xgt2/334.

[99] D. Nguyen, Model dependence study for ca cross section using ca and fe coefficients, https://hallaweb.jlab.org/dvcslog/Xgt2/336.

[100] D. Nguyen, Model dependence study for ca cross section using initial and updated cross section model, https://hallaweb.jlab.org/dvcslog/Xgt2/ 348.

[101] J. Ryckebusch, W. Cosyn, and M. Vanhalst, J. Phys. G42, 055104 (2015), 1405.3814.

[102] W. Cosyn, Private communication.

[103] CLAS, M. Duer et al., Nature 560, 617 (2018).

[104] J. Arrington et al., Phys. Rev. C86, 065204 (2012), 1206.6343.

[105] H. Gallagher, G. Garvey, and G. P. Zeller, Ann. Rev. Nucl. Part. Sci. 61, 355 (2011). 